\title{
WestVirginiaUniversity
}

THE RESEARCH REPOSITORY @ WVU

Graduate Theses, Dissertations, and Problem Reports

2002

\section{Mechanical characterization of aged recycled polymers and applications}

Jose Ricardo Basto

West Virginia University

Follow this and additional works at: https://researchrepository.wvu.edu/etd

\section{Recommended Citation}

Basto, Jose Ricardo, "Mechanical characterization of aged recycled polymers and applications" (2002). Graduate Theses, Dissertations, and Problem Reports. 1275.

https://researchrepository.wvu.edu/etd/1275

This Thesis is protected by copyright and/or related rights. It has been brought to you by the The Research Repository @ WVU with permission from the rights-holder(s). You are free to use this Thesis in any way that is permitted by the copyright and related rights legislation that applies to your use. For other uses you must obtain permission from the rights-holder(s) directly, unless additional rights are indicated by a Creative Commons license in the record and/ or on the work itself. This Thesis has been accepted for inclusion in WVU Graduate Theses, Dissertations, and Problem Reports collection by an authorized administrator of The Research Repository @ WVU. For more information, please contact researchrepository@mail.wvu.edu. 


\title{
MECHANICAL CHARACTERIZATION OF AGED RECYCLED POLYMERS AND APPLICATIONS
}

José Ricardo Basto M.

\author{
Thesis Submitted to the \\ College of Engineering and Mineral Resources \\ at West Virginia University \\ in partial fulfillment of the requirements \\ for the degree of
}

Master of Science

in

Civil Engineering

\author{
P.V. Vijay, Ph.D., Chair \\ Hota GangaRao, Ph.D., Co-Chair \\ Rakesh Gupta, Ph.D. \\ Department of Civil and Environmental Engineering \\ Morgantown, West Virginia \\ 2002
}

Keywords: Recycled Polymers, ABS, PC, PP, Tension, Compression, Bending, Hardness, Impact, Creep, Bonding, Aging, Highway Guardrails, Tires 


\section{ABSTRACT \\ Mechanical Characterization Of Aged Recycled Polymers And Applications}

\section{José Ricardo Basto M.}

The research focuses on the evaluation of mechanical properties (tension, bending, compression, impact, hardness and creep) of recycled acrylonitrile butadiene styrene (ABS) and polycarbonate (PC) thermoplastics obtained as electronic shredder residue (ESR) from computer housings. Three forms of polymers, i.e., virgin, blend of virgin and recycled, and 100\% recycled were investigated to establish their long term mechanical properties. Chopped and continuous glass fiber / fabric addition to the resins was evaluated to study the thermo-mechanical properties.

Mechanical characterization was carried out at freeze-thaw cycling aging. It was found that recycled polymers retain at least $70 \%$ of their tensile, bending and compressive strength after 18 months of aging under harsh environment (60 years in practice).

Offset block modules for highway guardrail systems were manufactured with recycled ABS and discarded rubber tires. Reinforced recycled plastic shapes were evaluated as possible post and rail for highway guardrail systems. Channel, trapezoidal, and box sections, and flat sheets made of recycled polypropylene (PP) and ABS were tested in tension, compression and bending.

Based on the manufacturing of laboratory specimens and test results, it is concluded that recycled polymers have significant potential for high-volume infrastructure and automotive applications. 
To my wife, María José "Chiquitaja" 


\section{ACKNOWLEDGEMENTS}

I wish to sincerely thank the Constructed Facilities Center at West Virginia University and expressly my advisors Dr. Hota GangaRao and Dr. P.V. Vijay. They gave me the opportunity to conduct my research and complete my Masters degree, and they provided me with valuable guidance and thoughts on my research. Thanks to Dr. Rakesh Gupta for serving on my committee and providing contributive suggestions.

My special recognition to the personnel in charge of the civil and chemical engineering departmental laboratories at West Virginia University, for their collaboration in running my experiments, and their patience in showing me the use of their facilities.

I am grateful to the U.S. DOE for funding this research. Thanks to Dr. Carl Irwin for assisting us during the research.

My sincere appreciation to Vijay Tripathy and all my research colleagues at WVU who offered me their unconditional help during my research. Special thanks to the Constructed Facilities Center staff who, in one way or another, contributed to the successfully completion of this research.

Heartfelt thanks to my family: Clara, Jose A., Patty and Dan who have always supported and encouraged me, and to my beloved partner and wife Maria Jose who experienced by my side the joys and difficulties of completing this thesis. Thanks to God for blessing me with the wonderful gift of life. 


\section{TABLE OF CONTENTS}

$\begin{array}{ll}\text { ABSTRACT } & \text { ii }\end{array}$

ACKNOWLEDGEMENTS

TABLE OF CONTENTS V V

LIST OF TABLES xi

LIST OF FIGURES $\quad$ xxi

\section{CHAPTER 1}

INTRODUCTION 1

1.1 General Remarks 1

1.2 Objectives 2

$\begin{array}{lll}1.3 & \text { Scope } & 3\end{array}$

1.3.1 Coupon Specimens from Conditioned Recycled Thermoplastics 3

1.3.2 Guardrail Post, Rail, and Offset Block 4

\section{CHAPTER 2}

LITERATURE REVIEW 8

2.1 Introduction $\quad 8$

$\begin{array}{lll}2.2 & \text { Recycling Aspects } & 10\end{array}$

2.3 Recycled Plastic Utilization 11

2.4 Previous Research on Plastics in Infrastructure 12

2.5 Conclusions 15 


\section{CHAPTER 3}

MATERIALS, PROCESS AND TEST PROCEDURE FOR CONDITIONED RECYCLED THERMOPLASTIC COUPONS 17

$\begin{array}{lll}3.1 & \text { Introduction } & 17\end{array}$

$\begin{array}{llr}3.2 & \text { Materials } & 18\end{array}$

$\begin{array}{lll}3.2 .1 & \text { Virgin Polymers } & 18\end{array}$

$\begin{array}{ll}\text { 3.2.2 Recycled Polymers } & 18\end{array}$

$\begin{array}{lll}\text { 3.2.3 Glass Fibers } & 18\end{array}$

$\begin{array}{lll}3.3 & \text { Manufacturing of Specimens } & 19\end{array}$

$\begin{array}{lll}\text { 3.3.1 Injection Molding Process } & 19\end{array}$

$\begin{array}{lll}3.4 & \text { Aging (conditioning) } & 22\end{array}$

$\begin{array}{lll}3.5 & \text { Testing } & 22\end{array}$

$\begin{array}{lll}3.5 .1 & \text { Tension Test } & 23\end{array}$

3.5.1.1 Test Specimen 23

3.5.1.2 Specimen Preparation $\quad 24$

3.5.1.3 Test Set-up and Procedure 24

$\begin{array}{lll}\text { 3.5.2 Bending Test } & 25\end{array}$

$\begin{array}{ll}\text { 3.5.2.1 Test Specimen } & 25\end{array}$

3.5.2.2 Specimen Preparation 26

3.5.2.3 Test Set-up and Procedure 26

$\begin{array}{ll}\text { 3.5.3 Compression Test } & 27\end{array}$

$\begin{array}{lll}\text { 3.5.3.1 Test Specimen } & 27\end{array}$

3.5.3.2 Specimen Preparation 28 
3.5.3.3 Test Set up and Procedure 28

3.5.4 Impact Test 29

$\begin{array}{lll}\text { 3.5.4.1 Test Specimen } & 29\end{array}$

3.5.4.2 Specimen Preparation 30

3.5.4.3 Test Set up and Procedure $\quad 30$

$\begin{array}{lll}\text { 3.5.5 Hardness Test } & 31\end{array}$

3.5.5.1 Test Specimen 31

3.5.5.2 Specimen Preparation 31

3.5.5.3 Test Set up and Procedure $\quad 31$

$\begin{array}{lll}\text { 3.5.6 Creep Test } & 32\end{array}$

3.5.6.1 Test Specimen $\quad 32$

3.5.6.2 Specimen Preparation 32

3.5.6.3 Test Set up and Procedure $\quad 34$

\section{CHAPTER 4}

MATERIALS, MANUFACTURING AND TEST PROCEDURE FOR

GUARDRAIL POST, RAIL AND OFFSET BLOCK 35

$\begin{array}{lll}4.1 & \text { Introduction } & 35\end{array}$

$\begin{array}{lll}4.2 & \text { Materials } & 36\end{array}$

4.2.1 Recycled Polymers 36

$\begin{array}{lll}\text { 4.2.2 Glass Fibers } & 37\end{array}$

$\begin{array}{lll}\text { 4.2.3 } & \text { Rubber Tires } & 37\end{array}$

4.3 Manufacturing of Offset Block 37 
$\begin{array}{lll}\text { 4.3.1 Molding Procedure } & 38\end{array}$

4.3.2 Small Block Molding 41

4.3.3 Heat Transfer Aspects 45

4.3.4 Final Product - Small Scale 46

4.3.5 Final Product - Prototype 48

$\begin{array}{ll}\text { 4.3.6 Final Molding Procedure } & 50\end{array}$

4.3.6.1 Molding of Tabs $\quad 50$

4.3.6.2 Molding of Offset Block Module 52

$\begin{array}{lll}4.4 & \text { Testing } & 57\end{array}$

$\begin{array}{lll}\text { 4.4.1 Bending Test } & 57\end{array}$

$\begin{array}{lll}\text { 4.4.1.1 Test Specimens } & 57\end{array}$

4.4.1.2 Specimen Preparation $\quad 59$

4.4.1.3 Test Set up and Procedure $\quad 62$

$\begin{array}{lll}\text { 4.4.2 Tension Test } & 64\end{array}$

4.4.2.1 Test Specimen $\quad 64$

4.4.2.2 Specimen Preparation 66

$\begin{array}{lll}\text { 4.4.2.3 Test Set up and Procedure } & 67\end{array}$

$\begin{array}{lll}\text { 4.4.3 Compression Test } & 68\end{array}$

$\begin{array}{lll}\text { 4.4.3.1 Test Specimen } & 68\end{array}$

4.4.3.2 Specimen Preparation $\quad 68$

4.4.3.3 Test Set up and Procedure $\quad 68$

4.4.4 Compression Test of Rubber-Wood Offset Block Model 68

$\begin{array}{lll}\text { 4.4.4.1 Test Specimen } & 68\end{array}$ 
4.4.4.2 Specimen Preparation $\quad 69$

4.4.4.3 Test Set up and Procedure $\quad 69$

4.4.5 Tests on Wood from Rubber-Wood Block Model 69

$\begin{array}{lll}\text { 4.4.5.1 Test Specimen } & 69\end{array}$

$\begin{array}{lll}\text { 4.4.5.2 Specimen Preparation } & 70\end{array}$

$\begin{array}{lll}\text { 4.4.5.3 Test Set up and Procedure } & 70\end{array}$

4.4.6 Bond Strength Test for Rubber-ABS Interface 72

$\begin{array}{lll}\text { 4.4.6.1 Test Specimen } & 72\end{array}$

4.4.6.2 Specimen Preparation $\quad 74$

4.4.6.3 Test Set-up and Procedure $\quad 75$

$\begin{array}{ll}4.5 \text { Conclusions } & 75\end{array}$

\section{CHAPTER 5}

TEST RESULTS, ANALYSIS AND DISCUSSIONS ON CONDITIONED

RECYCLED THERMOPLASTICS 76

$\begin{array}{lll}5.1 & \text { Introduction } & 76\end{array}$

$\begin{array}{lll}5.2 & \text { Tension Test } & 76\end{array}$

$\begin{array}{lll}\text { 5.2.1 Results on ABS } & 76\end{array}$

$\begin{array}{ll}\text { 5.2.2 Analysis and Discussion on ABS } & 78\end{array}$

$\begin{array}{lll}\text { 5.2.3 Results on PC } & 80\end{array}$

5.2.4 Analysis and Discussion on PC 82

$\begin{array}{lll}5.3 & \text { Bending Test } & 84\end{array}$

$\begin{array}{lll}\text { 5.3.1 Results on ABS } & 84\end{array}$ 
5.3.2 Analysis and Discussion on ABS 85

$\begin{array}{lll}\text { 5.3.3 Results on PC } & 87\end{array}$

$\begin{array}{ll}\text { 5.3.4 Analysis and Discussion on PC } & 89\end{array}$

$\begin{array}{lll}5.4 & \text { Compression Test } & 91\end{array}$

$\begin{array}{lll}\text { 5.4.1 Results on ABS } & 91\end{array}$

5.4.2 Analysis and Discussion on ABS 92

$\begin{array}{lll}\text { 5.4.3 Results on PC } & 94\end{array}$

5.4.4 Analysis and Discussion on PC 96

$\begin{array}{lll}5.5 & \text { Impact Test } & 98\end{array}$

$\begin{array}{lll}\text { 5.5.1 Results on ABS } & 98\end{array}$

5.5.2 Analysis and Discussion on ABS 99

$\begin{array}{lll}\text { 5.5.3 Results on PC } & 99\end{array}$

$\begin{array}{ll}\text { 5.5.4 Analysis and Discussion on PC } & 100\end{array}$

$\begin{array}{lll}5.6 & \text { Hardness Test } & 101\end{array}$

$\begin{array}{lll}\text { 5.6.1 Results on ABS } & 101\end{array}$

$\begin{array}{ll}\text { 5.6.2 Analysis and Discussion on ABS } & 102\end{array}$

$\begin{array}{lll}\text { 5.6.3 Results on PC } & 103\end{array}$

$\begin{array}{lll}\text { 5.6.4 Analysis and Discussion on PC } & 104\end{array}$

$\begin{array}{lll}5.7 & \text { Creep Test } & 105\end{array}$

$\begin{array}{lll}5.7 .1 & \text { Results on ABS } & 105\end{array}$

$\begin{array}{ll}\text { 5.7.2 Analysis on ABS } & 107\end{array}$

$\begin{array}{lll}\text { 5.7.3 Results on PC } & 108\end{array}$

$\begin{array}{lll}\text { 5.7.4 Analysis on PC } & 110\end{array}$ 


\section{CHAPTER 6}

TEST RESULTS, ANALYSIS AND DISCUSSIONS ON GUARDRAIL POST,

$\begin{array}{ll}\text { RAIL AND OFFSET BLOCK } & 113\end{array}$

$\begin{array}{lll}6.1 & \text { Introduction } & 113\end{array}$

6.2 Recycled Polypropylene Channel Section 113

6.2.1 Test Results on Recycled Polypropylene Channel Section 113

6.2.2 Analysis and Discussion of Test Results on Recycled

$\begin{array}{ll}\text { Polypropylene Channel Section } & 115\end{array}$

6.3 Recycled Polypropylene Trapezoidal Section 116

6.3.1 Test Results on Recycled Polypropylene Trapezoidal Section 116

6.3.2 Analysis and Discussion of Test Results on Recycled

$\begin{array}{ll}\text { Polypropylene Trapezoidal Section } & 119\end{array}$

$\begin{array}{lll}6.4 & \text { Recycled ABS Box Sections } & 120\end{array}$

6.4.1 Test Results on Recycled ABS Box Section 120

6.4.2 Analysis and Discussion of Test Results on Recycled ABS Box

$\begin{array}{ll}\text { Section } & 123\end{array}$

$\begin{array}{ll}\text { 6.5 Plates from Recycled ABS Sheets } & 125\end{array}$

6.5.1 Test Results on Plates with Recycled ABS Sheets 125

6.5.2 Analysis and Discussion of Plate Test Results from Recycled

$\begin{array}{ll}\text { ABS Sheets } & 129\end{array}$

6.6 Strips from Recycled ABS Belt-Type Specimens 129

6.6.1 Test Results on Strips from Recycled ABS Belt-Type Specimens 129 
6.6.2 Analysis and Discussion of Strips from Recycled ABS Belt-Type Specimens

6.7 Compression Test of Rubber-Wood Offset Block Model

6.7.1 Results of Compression Test of Rubber-Wood Offset Block Model

6.7.2 Analysis and Discussion of Compression Test of Rubber-Wood Offset Block Model

6.8 Tests on Wood from Rubber-Wood Offset Block Model

6.8.1 Results of Tests on Wood from Rubber-Wood Offset Block Model

6.8.2 Analysis and Discussion of Tests on Wood from Rubber-Wood Block Model

6.9 Bond Strength Test on Rubber-ABS Interface 136

6.9.1 Results of Bond Strength Test on Rubber-ABS Interface 136

6.9.2 Analysis and Discussion of Bond Strength Test on Rubber-ABS Interface

\section{CHAPTER 7}

SUMMARY, CONCLUSIONS AND RECOMMENDATIONS FOR COUPON

SPECIMENS FROM CONDITIONED RECYCLED THERMOPLASTICS 140

$\begin{array}{lll}7.1 & \text { Introduction } & 140\end{array}$

7.2 Summary of Tension Test Results 141

7.3 Summary of Bending Test Results 141 
7.4 Summary of Compression Test Results

7.5 Summary of Impact Test Results

7.6 Summary of Hardness Test Results 144

$\begin{array}{lll}\text { 7.7 Summary of Creep Test Results } & 144\end{array}$

$\begin{array}{lll}\text { 7.8 Conclusions and Recommendations } & 145\end{array}$

\section{CHAPTER 8}

SUMMARY, CONCLUSIONS AND RECOMMENDATIONS FOR GUARDRAIL POST, RAIL AND OFFSET BLOCK 148

$\begin{array}{lll}8.1 & \text { Introduction } & 148\end{array}$

$\begin{array}{lll}\text { 8.2 Summary of Offset Block Manufacturing } & 148\end{array}$

8.3 Summary of Recycled Polypropylene Channel Section $\quad 150$

8.4 Summary of Recycled Polypropylene Trapezoidal Section 150

8.5 Summary of Recycled and Virgin ABS Box Sections 151

8.6 Summary of Recycled ABS Sheets 153

8.7 Summary of Recycled ABS Belt-Type Material 154

8.8 Summary of Rubber-Wood Offset Block Model 155

8.9 Summary of Tests on Wood Used in Rubber - Wood Model 155

8.10 Summary of Bond Strength on Rubber-ABS Interface 155

8.11 Conclusions and Recommendations 156

$\begin{array}{ll}\text { REFERENCES } & 163\end{array}$ 
APPENDIX A

TENSION TEST RESULTS FOR CONDITIONED SPECIEMENS

APPENDIX B

BENDING TEST RESULTS FOR CONDITIONED SPECIEMENS

168

APPENDIX C

COMPRESSION TEST RESULTS FOR CONDITIONED SPECIEMENS

APPENDIX D

IMPACT TEST RESULTS FOR CONDITIONED SPECIEMENS

APPENDIX E

HARDNESS TEST RESULTS FOR CONDITIONED SPECIEMENS

\author{
APPENDIX F \\ COMPARISON CHARTS OF TEST RESULTS ON CONDITIONED \\ RECYCLED COUPONS
}

APPENDIX G

CREEP TEST RESULTS

APPENDIX H

TEST RESULTS FOR FIBER REINFORCED PLASTIC SPECIMENS FOR HIGHWAY APPLICATIONS 


\section{APPENDIX I}

THEORETICAL CALCULATION OF TENSILE MODULUS FOR

RANDOMLY ORIENTED CHOPPED FIBER REINFORCED

PLASTICS

216 


\section{LIST OF TABLES}

Table 1.1 Types of Coupon Specimens from Conditioned Recycled Thermoplastics, Tests and Aging Times 3

Table 1.2 Creep Test Specimens 4

Table 1.3 Guardrail Post, Rail and Offset Block Specimens Tested 6

Table 2.1 Types of Recyclable Materials 9

Table 3.1 Injection Molding Process for ABS Samples (CFC-WVU and Owens Corning Partnership) 20

Table 3.2 Injection Molding Process for PC Samples (CFC-WVU and $\begin{array}{ll}\text { PPG Partnership) } & 20\end{array}$

Table 3.3 Types of ABS Specimens 21

Table 3.4 Types of PC Specimens 21

Table 3.5 Coupon Dimension and Test Procedure as per ASTM Standard 22

Table 4.1 Types of Bending Specimen for Guardrail Systems 57

Table 5.1 Tensile Strength Variations in Aged ABS Specimens 77

Table 5.2 Tensile Stiffness Variations in Aged ABS Specimens 77

Table 5.3 Tensile Strength Variations in Aged PC Specimens 80

Table 5.4 Tensile Stiffness Variations in Aged PC Specimens 81

Table 5.5 Bending Strength Variations in Aged ABS Specimens 84

Table 5.6 Bending Stiffness Variations in Aged ABS Specimens 85

Table 5.7 Bending Strength Variations in Aged PC Specimens 88

Table 5.8 Bending Stiffness Variations in Aged PC Specimens 88

Table 5.9 Compressive Strength Variations in Aged ABS Specimens 91

Table 5.10 Compressive Stiffness Variations in Aged ABS Specimens 92

Table 5.11 Compressive Strength Variations in Aged PC Specimens 94

Table 5.12 Compressive Stiffness Variations in Aged PC Specimens 95

Table 5.13 Impact Strength Variations in ABS Specimens 98

Table 5.14 Impact Strength Variations in Aged PC Specimens 100

Table 5.15 Hardness Index Variations in Aged ABS Specimens 102

Table 5.16 Hardness Index Variations in Aged PC Specimens 103 
Table 5.17 Creep Test Results for 20\% Sustained Load on ABS Specimens

Table 5.18 Creep Test Results for 50\% Sustained Load on ABS Specimens 106

Table 5.19 Creep Test Results for 20\% Sustained Load on PC

$\begin{array}{ll}\text { Specimens at } 809 \text { Days } & 108\end{array}$

Table 5.20 Creep Test Results for 50\% Sustained Load on PC

Specimens at 809 Days

109

Table 6.1 Maximum Tensile and Compressive Stresses and Stiffnesses of

Polypropylene Channel Section Under Three Point Bending Test 114

Table 6.2 Maximum Tensile and Compressive Stresses and Stiffnesses of

Polypropylene Trapezoidal Section Under Three Point

Bending Test

Table 6.3 Maximum Tensile Stresses and Stiffnesses on Polypropylene

Plates from Trapezoidal Section - Tension Test

Table 6.4 Fiber Volume Fraction and Dimensional Properties for ABS

Box Sections

Table 6.5 Maximum Tensile and Compressive Stresses and Stiffnesses

in Box Sections

Table 6.6 Maximum Tensile Stress and Stiffness for Recycled ABS

Box Section - Tension Test

Table 6.7 Maximum Tensile and Compressive Stresses and Stiffnesses

in Recycled ABS Plates - Three Point Bending Test

Table 6.8 Maximum Tensile Stress and Stiffness on Plates from ABS

Sheets - Tension Test

Table 6.9 Maximum Compressive Stress and Stiffness on Plates from ABS

Sheets

Table 6.10 Maximum Stress and Stiffness of Strips from ABS Belt -Type Specimens - Tension Test

Table 6.11 Maximum Compressive Stress and Stiffness of Wooden Blocks from Rubber - Wood Block Model - Compression Parallel to Grain Test

Table 6.12 Maximum Compressive Stress and Stiffness of Wood Blocks 
from Rubber - Wood Block Model - Compression Perpendicular to Grain Test

Table 6.13 Bond Strength Test Results ABS-Rubber Interface

Table 8.1 Mechanical Properties of Recycled Trapezoidal Section

Table 8.2 Mechanical Properties of Recycled and Virgin Box Sections

Table 8.3 Mechanical Properties of Recycled ABS Sheets

Table 8.4 Mechanical Properties of Wood from Rubber-Wood Model

Table A.1 Tension Test Results for 2 Months of Aging

Table A.2 Tension Test Results for 4 Months of Aging

Table A.3 Tension Test Results for 10 Months of Aging

Table A.4 Tension Test Results for 18 Months of Aging

Table B.1 Bending Test Results for 2 Months of Aging 168

Table B.2 Bending Test Results for 4 Months of Aging 168

Table B.3 Bending Test Results for 10 Months of Aging 169

Table B.4 Bending Test Results for 18 Months of Aging 170

Table C.1 Compression Test Results for 2 Months of Aging 171

Table C.2 Compression Test Results for 4 Months of Aging 172

Table C.3 Compression Test Results for 18 Months of Aging 173

Table D.1 Impact Test Results for 2 Months of Aging 174

Table D.2 Impact Test Results for 4 Months of Aging 174

Table D.3 Impact Test Results for 10 Months of Aging 175

Table D.4 Impact Test Results for 18 Months of Aging 176

Table E.1 Hardness Test Results for 2 Months of Aging 177

Table E.2 Hardness Test Results for 4 Months of Aging 177

Table E.3 Hardness Test Results for 10 Months of Aging 178

Table E.4 Hardness Test Results for 18 Months of Aging 179

Table F.1 Tensile Strength Variations in Aged ABS Specimens 180

Table F.2 Tensile Stiffness Variations in Aged ABS Specimens 181

Table F.3 Tensile Strength Variations in Aged PC Specimens 182

Table F.4 Tensile Stiffness Variations in Aged PC Specimens 183

Table F.5 Bending Strength Variations in Aged ABS Specimens 184 
Table F.6 Bending Stiffness Variations in Aged ABS Specimens 185

Table F.7 Bending Strength Variations in Aged PC Specimens 186

Table F.8 Bending Stiffness Variations in Aged PC Specimens 187

Table F.9 Compressive Strength Variations in Aged ABS Specimens 188

Table F.10 Compressive Stiffness Variations in Aged ABS Specimens 189

Table F.11 Compressive Strength Variations in Aged PC Specimens 190

Table F.12 Compressive Stiffness Variations in Aged PC Specimens 191

Table F.13 Impact Strength Variations in Aged ABS Specimens 192

Table F.14 Impact Strength Variations in Aged PC Specimens 193

Table F.15 Hardness Index Variations in Aged ABS Specimens 194

Table F.16 Hardness Index Variations in Aged PC Specimens 195

Table G.1 Creep Test Results for 100\% Recycled and Blend ABS without Fibers at 20\% Sustained Loading 196

Table G.2 Creep Test Results for Virgin ABS without Fibers; Virgin, Blend, and $100 \%$ Recycled with Fibers at 20\% Sustained Loading

Table G.3 Creep Test results for 100\% Recycled and Blend ABS without Fibers 50\% Sustained Loading 202

Table G.4 Creep Test Results for Virgin ABS without Fibers; Virgin, Blend, and $100 \%$ Recycled with Fibers at 50\% Sustained Loading

Table G.5 Creep Test Results for Virgin, Blend and 100\% Recycled PC with and without Fibers at 20\% Sustained Loading 207

Table G.6 Creep Test Results for Virgin, Blend and 100\% Recycled PC with and without Fibers at 50\% Sustained Loading 209

Table H.1 Recycled Polypropylene Channel Section Bending Test Results 211

Table H.2 Recycled Polypropylene Trapezoidal Section Bending Test Results

Table H.3 Plates from Recycled Polypropylene Trapezoidal Section Tension Test Results

Table H.4 Recycled ABS Box Section Bending Test Results 212

Table H.5 Virgin Vinylester Box Section 3-Point Bending Test Results 212

Table H.6 Recycled ABS Box Section Tension Test Results 212 
Table H.7 Recycled ABS Short Plates Cut from Sheets Bending Test Results

Table H.8 Recycled ABS Long Plates Cut from Sheets Bending Test Results

Table H.9 Recycled ABS Sheets Tension Test Results 213

Table H.10 Recycled ABS Sheets Compression Test Results 213

Table H.11 Recycled ABS Belt-Type Specimens Tension Test Results 214

Table H.12 Wooden Blocks from Rubber-Wood Block Model Compression Test Results

Table H.13 Wooden Blocks from Rubber-Wood Block Model Impact Test Results

Table H.14 ABS-Rubber Pull-Out Test Results 215

Table H.15 Fiber Reinforced Plastics Ignition Test Results 215 


\section{LIST OF FIGURES}

Figure 1.1 Typical Guardrail Joint Configuration and Modular Offset Block 5

Figure 3.1 Conditioning (Aging) Temperature Cycle 23

Figure 3.2 Tension Specimen Dimensions 24

Figure 3.3 Close-up of Tension Test Set-up with Extensometer Attached 25

Figure 3.4 Test Set-up for Tension Test 25

$\begin{array}{lll}\text { Figure 3.5 Test Set-up for Bending Test } & 27\end{array}$

Figure 3.6 Close-up of Bending Test 27

Figure 3.7 Test Set-up for Compression Test - Instron Machine 28

Figure 3.8 Close-up for Compression Test 29

Figure 3.9 Impact Specimen Dimensions 29

Figure 3.10 Test Set-up for Impact Test 30

Figure 3.11 Test Set-up for Hardness Test 31

Figure 3.12 Creep Specimen 33

Figure 3.13 Creep Specimen Set-up 33

Figure 3.14 Creep Test Set-up 34

Figure 4.1 ABS Recycled Pellets for Offset Block Manufacturing 37

Figure 4.2 Schematic of Compression Molding 38

Figure 4.3 Mold and Pellets for Pressure Molding 39

$\begin{array}{lll}\text { Figure } 4.4 \text { Molding Set-up } & 40\end{array}$

Figure 4.5 Pressure Molding Machine During Molding Process 40

Figure 4.6 Solid Recycled ABS Small Block 42

Figure $4.7 \quad$ Filled Small Blocks $\quad 42-43$

Figure 4.8 Two Stage Molding Process 44

Figure 4.9 Rubber-Wood Model 45

Figure 4.10 Pre-molded ABS Tabs and Rubber Core 47

Figure 4.11 Specimen Molded With Inadequate Amount of Pellets and Solidified After Melting Under Pressure $\quad 47$

Figure 4.12 Pre-heated Molded Block 48

Figure 4.13 Offset Block Dimensions 48 
Figure 4.14 Modular Offset Block

Figure 4.15 Tab Mold, Prepared for Molding $\quad 50$

Figure 4.16 Mold for Tab Manufacturing With Recycled Pellets 51

Figure 4.17 Molding of Tabs 51-52

Figure 4.18 Mold Prepared for Module Manufacturing

(Wrapped Core Also Shown) 53

Figure 4.19 Rubber Tire Strips for Module Core 53

Figure 4.20 ABS Tabs Covering Rubber Core $\quad 54$

Figure 4.21 Wrapped Rubber-ABS Core 54

Figure 4.22 Filled Mold 55

Figure 4.23 Pre-heating Procedure 56

Figure 4.24 Molded Offset Block Module 56

Figure 4.25 Channel Section Dimensions of Propylene Specimen 58

Figure 4.26 Trapezoidal Section Dimensions of Propylene Specimen 58

Figure 4.27 Box Section Dimensions of ABS Specimen 58

Figure 4.28 Short Plate and Long Bending Specimens (With Strain Gage)

Cut from Recycled ABS Sheets (Also Shown) 59

Figure 4.29 Strain Gage Locations for Bending Test on Channel Section $\quad 60$

Figure 4.30 Strain Gage Locations for Bending Test on Trapezoidal Section $\quad 60$

Figure 4.31 Strain Gage Locations for Bending Test on Box Sections $\begin{array}{ll}\text { (Midspan) } & 60\end{array}$

Figure 4.32 Non Wrapped and Wrapped Box Specimens for Bending Test 61

Figure 4.33 Wooden Stiffeners at Ends of Channel Section 62

Figure 4.34 Bending Test Set-up: Universal Testing Machine and Data Acquisition System $\quad 62$

Figure 4.35 Channel Section Set-up for Bending Test 63

Figure 4.36 Trapezoidal Section Bending Test Set-up and Failure Mode 63

Figure 4.37 Wrapped Box Section Three and Four Point Bending Test Set-up 63

Figure 4.38 Virgin Vinylester Box Section Three Point Bending Test Set-up 64

Figure 4.39 Recycled ABS Half Sheet Specimen Three Point Bending Test Set-up $\quad 64$ 
Figure 4.40 Polypropylene Strip Specimen from Trapezoidal Sections Used for Tension Tests (Before and After Testing) 65

Figure 4.41 ABS Strip Tension Specimen from Belt Type (Flexible) Product and from Sheets (Last Two on the Right) 65

Figure 4.42 ABS Belt Type (Flexible) Product 66

Figure 4.43 Strip Type Specimens Tension Test Set-up 67

Figure 4.44 Box Specimen Tension Test Set-up 68

Figure 4.45 Compression Test on Rubber-Wood Offset Block Model 69

Figure 4.46 Tested Wooden Impact Specimens 70

Figure 4.47 Compression Parallel to Grain Test Set-up 71

Figure 4.48 Compression Perpendicular to Grain Test Set-up 71

Figure 4.49 Compression Molding of Bond Specimen 73

$\begin{array}{lll}\text { Figure 4.50 Rubber Tire-ABS Bond Specimen } & 74\end{array}$

Figure 4.51 Bond Specimen Apparatus for Pull-out Test 74

$\begin{array}{lll}\text { Figure 4.52 Bond Test Set-up } & 75\end{array}$

$\begin{array}{lll}\text { Figure 5.1 Tensile Strength in Aged ABS Specimens } & 77\end{array}$

$\begin{array}{lll}\text { Figure 5.2 Tensile Stiffness in Aged ABS Specimens } & 78\end{array}$

Figure 5.3 Tensile Strength in Aged PC Specimens 81

$\begin{array}{lll}\text { Figure 5.4 Tensile Stiffness in Aged PC Specimens } & 82\end{array}$

Figure 5.5 Bending Strength in Aged ABS Specimens 84

Figure 5.6 Bending Stiffness in Aged ABS Specimens 85

$\begin{array}{lll}\text { Figure 5.7 Bending Strength in Aged PC Specimens } & 88\end{array}$

$\begin{array}{lll}\text { Figure 5.8 Bending Stiffness in Aged PC Specimens } & 89\end{array}$

Figure 5.9 Compressive Strength in Aged ABS Specimens 91

Figure 5.10 Compressive Stiffness in Aged ABS Specimens 92

Figure 5.11 Compressive Strength in Aged PC Specimens 95

Figure 5.12 Compressive Stiffness in Aged PC Specimens 96

Figure 5.13 Impact Strength in Aged ABS Specimens 98

$\begin{array}{lll}\text { Figure 5.14 Impact Strength in Aged PC Specimens } & 100\end{array}$

Figure 5.15 Hardness Index in Aged ABS Specimens 102

Figure 5.16 Hardness Index in Aged PC Specimens 104 
Figure 5.17 Creep Test on ABS Specimens for 20\% Sustained Load 106

Figure 5.18 Creep Test on ABS Specimens for 50\% Sustained Load 107

Figure 5.19 Creep Test on PC Specimens for 20\% Sustained Load 109

Figure 5.20 Creep Test on PC Specimens for 50\% Sustained Load 110

Figure 6.1 Distances from Centroid of Channel Section to Locations of Strain Gages

Figure 6.2 Compressive and Tensile Stresses in Recycled Polypropylene

Channel Section Under Three Point Bending Test 115

Figure 6.3 Distances from Centroid of Trapezoidal Section to Locations of Strain Gages

Figure 6.4 Compressive and Tensile Bending Stresses on Recycled Polypropylene Trapezoidal Section Under Three Point Bending Test

Figure 6.5 Linear Portion of Stress vs. Strain for Tension Tests on Recycled Polypropylene Plates from Trapezoidal Section

Figure 6.6 Box Sections (from Left to Right) Recycled ABS, Wrapped Recycled ABS and Virgin Vinylester

Figure 6.7 Compressive and Tensile Bending Stresses in Recycled ABS Box Section Under Three and Four Point Bending Test

Figure 6.8 Tensile Stress vs. Strain for Tension Tests on Recycled ABS Box Section

Figure 6.9 Tensile and Compressive Bending Stress in Recycled ABS Plates and Under Three Point Bending Test

Figure 6.10 Linear Portion of Stress vs. Strain for Tension Tests on ABS

Plates from Recycled ABS Sheets

Figure 6.11 Compressive Stress vs. Strain for Compression Tests on Plates from Recycled ABS Sheets

Figure 6.12 Tensile Stress vs. Strain of Recycled ABS Strips from Belt-Type Specimens

Figure 6.13 Compressive Stress vs. Strain for Compression Parallel to Grain 
Tests on Wooden Blocks from Rubber - Wood Block Model

Figure 6.14 Stress vs. Strain for Compression Perpendicular to Grain Tests on

Wooden Blocks from Rubber - Wood Block Model

Figure 6.15 Comparison of Impact Strength Between Virgin and

Combinations of Recycled ABS and PC, and Wood

Figure 6.16 Bond Stress vs. Deflection for Pull Out Tests on ABS - Rubber

Interface

Figure 6.17 Results of Bond Strength Tests on ABS - Rubber Interface

With and Without Primer for Different Molding (Curing)

Duration

Figure F.1 Tensile Strength in Aged ABS Specimens

Figure F.2 Tensile Stiffness in Aged ABS Specimens

Figure F.3 Tensile Strength in Aged PC Specimens

$\begin{array}{lll}\text { Figure F.4 Tensile Stiffness in Aged PC Specimens } & 183\end{array}$

$\begin{array}{lll}\text { Figure F.5 Bending Strength in Aged ABS Specimens } & 184\end{array}$

$\begin{array}{lll}\text { Figure F.6 Bending Stiffness in Aged ABS Specimens } & 185\end{array}$

Figure F.7 Bending Strength in Aged PC Specimens 186

$\begin{array}{lll}\text { Figure F.8 Bending Stiffness in Aged PC Specimens } & 187\end{array}$

Figure F.9 Compressive Strength in Aged ABS Specimens 188

Figure F.10 Compressive Stiffness in Aged ABS Specimens 189

Figure F.11 Compressive Strength in Aged PC Specimens 190

Figure F.12 Compressive Stiffness in Aged PC Specimens 191

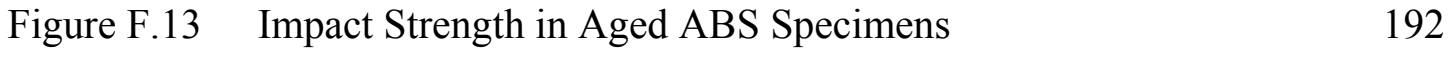

Figure F.14 Impact Strength in Aged PC Specimens 193

Figure F.15 Hardness Index in Aged ABS Specimens 194

Figure F.16 Hardness Index in Aged PC Specimens 195 


\section{CHAPTER 1}

\section{INTRODUCTION}

\subsection{General Remarks}

Thermoplastics such as acrylonitrile-butadiene styrene (ABS) and polycarbonate (PC) are widely used to manufacture housings for small appliances (e.g., calculators, telephones, computers, etc.) as well as large equipment (e.g., automobiles). Development of new technologies and their applications make these appliances obsolete within a few years after their introduction into the consumer markets. One such obsolescence is the personal computers, which have a fast paced introduction into the consumer market. About 25 million computers are made in America each year and it is predicted that by 2005 the number of obsolete computers per year will exceed the number of new computers produced by $18 \%$ (Riverdeep, 2002). Recycling of polymers from discarded plastic housings of appliances and equipment, especially personal computers can be economical and environmental friendly.

In order to add value to recycled electronic (computer casings) shredder residue (ESR), it is essential to blend polymer ESR with virgin thermoplastics and further improve their properties with glass fiber addition, thus resulting in energy savings as well as substantial reductions in landfill space. Because of improved thermo-mechanical properties, structural and non-structural systems such as highway guardrail systems, automobile bumpers, headlight housings and other applications can be manufactured economically using blended recycled and virgin polymers. However, a comprehensive 
understanding of thermo-mechanical characterization is essential before developing a wide range of applications for recyclable materials.

\subsection{Objectives}

This research is a sequel to the research conducted by the Constructed Facilities Center at West Virginia University (CFC-WVU) on mechanical property characterization of recycled thermoplastics (Bargo, 2000). Long-term mechanical property retention and durability were evaluated for two types of thermoplastics, i.e., ABS and PC (virgin, blend of virgin and recycled, and $100 \%$ recycled) resins reinforced with glass fibers. Data collection of creep behavior was continued for additional 2 years in this research and those results are reported herein.

The objectives of this research are to:

- Evaluate long-term strength and stiffness retention of virgin/recycled ESR-ABS and ESR-PC polymers under tension, compression, bending, impact and hardness with and without glass fibers.

- Characterize creep behavior of glass fiber reinforced ABS and PC polymers that are virgin, blends of virgin and recycled, and 100\% recycled ABS and PC. Continue the tests initiated by the CFC-WVU (Bargo, 2000) and analyze the results.

- Manufacture offset blocks for highway guardrail applications using recycled polymer composites and discarded tire strips.

- Evaluate bending, tensile and compressive properties of channel, trapezoidal and box section coupon specimens made of recycled polymers. 


\subsection{Scope}

\subsubsection{Coupon Specimens from Conditioned Recycled Thermoplastics}

Based on availability, mechanical and manufacturing suitability, and cost issues, $\mathrm{ABS}$ and PC based polymers with and without chopped glass fibers were used in this research. Tests were carried out to evaluate tension, bending, compression, impact and hardness properties. Mechanical properties were evaluated at 2, 4, 10 and 18 months of aging. A total of $340 \mathrm{ABS}$ and PC specimens were tested as part of this research. A summary of tests and specimen characteristics is shown in Table 1.1.

Table 1.1 Types of Coupon Specimens from Conditioned Recycled Thermoplastics, Tests and Aging Times

\begin{tabular}{|c|c|c|c|c|c|c|c|c|c|c|c|c|c|c|c|c|}
\hline \multirow{4}{*}{ Test } & \multicolumn{16}{|c|}{ Number of Tested Samples and Aging Times } \\
\hline & \multicolumn{4}{|c|}{ 2months } & \multicolumn{4}{|c|}{4 months } & \multicolumn{4}{|c|}{10 months } & \multicolumn{4}{|c|}{18 months } \\
\hline & \multicolumn{2}{|c|}{$\begin{array}{l}\text { With } \\
\text { Fibers }\end{array}$} & \multicolumn{2}{|c|}{$\begin{array}{l}\text { W/out } \\
\text { Fibers }\end{array}$} & \multicolumn{2}{|c|}{$\begin{array}{l}\text { With } \\
\text { Fibers }\end{array}$} & \multicolumn{2}{|c|}{$\begin{array}{l}\text { W/out } \\
\text { Fibers }\end{array}$} & \multicolumn{2}{|c|}{$\begin{array}{l}\text { With } \\
\text { Fibers }\end{array}$} & \multicolumn{2}{|c|}{$\begin{array}{l}\text { W/out } \\
\text { Fibers }\end{array}$} & \multicolumn{2}{|c|}{$\begin{array}{l}\text { With } \\
\text { Fibers }\end{array}$} & \multicolumn{2}{|c|}{$\begin{array}{l}\text { W/out } \\
\text { Fibers }\end{array}$} \\
\hline & 党 & $\underline{L}$ & a & $\underline{\underline{2}}$ & 2 & $£$ & 党 & $\underline{\underline{2}}$ & 2 & $\underline{Z}$ & a & $\underline{\underline{z}}$ & 党 & $\underline{Z}$ & a & $\underline{2}$ \\
\hline Tension & 3 & 3 & 3 & 3 & 3 & 3 & 3 & 3 & 6 & 6 & 5 & 5 & 5 & 6 & 5 & 6 \\
\hline Bending & 3 & 3 & 2 & 3 & 3 & 3 & 3 & 3 & 6 & 6 & 6 & 6 & 6 & 6 & 6 & 6 \\
\hline Compression & 4 & 6 & 6 & 6 & 5 & 5 & 4 & 3 & - & - & - & - & 6 & 6 & 6 & 6 \\
\hline Impact & 3 & 3 & - & - & 3 & 3 & 3 & 3 & 6 & 3 & 7 & 3 & 6 & 6 & 11 & 6 \\
\hline Hardness & 3 & 3 & 3 & 3 & 3 & 3 & 3 & 3 & 6 & 6 & 6 & 6 & 6 & 6 & 6 & 6 \\
\hline Total & 16 & 18 & 14 & 15 & 17 & 17 & 16 & 15 & 24 & 21 & 24 & 20 & 29 & 30 & 34 & 30 \\
\hline $\begin{array}{l}\text { Grand } \\
\text { Total }\end{array}$ & & & & & & & & & & & & & & & & 340 \\
\hline
\end{tabular}

In addition, monitoring of creep in ABS and PC specimens started by the CFC-

WVU (Bargo, 2000) was continued. A total of 24 specimens were evaluated under creep as shown in Table 1.2. 
Table 1.2 Creep Test Specimens

\begin{tabular}{|l|c|c|}
\hline \multirow{2}{*}{ Type of creep load } & \multicolumn{2}{|c|}{ Number of specimens } \\
\cline { 2 - 3 } & ABS & PC \\
\hline 20 \% sustained load & 6 & 6 \\
\hline 50\% sustained load & 6 & 6 \\
\hline Total & 12 & 12 \\
\hline Grand Total & \multicolumn{2}{|c|}{24} \\
\hline
\end{tabular}

\subsubsection{Guardrail Post, Rail and Offset Block}

Offset block to be used between soil and post in a guardrail system was developed in this study. Manufacturing issues of these components such as shape, size and amount of recycled pellets, molding temperature, time and pressure were identified and characterized for mass production purposes.

Mechanical properties such as tension, bending and compression of recycled polypropylene channel section and trapezoidal section, and recycled ABS box section were also evaluated. This characterization will help in determining the possibility of manufacturing these shapes using recycled polymers for use as rails and posts in highway guardrail systems. To obtain optimized shapes, design, and manufacturing processes have to be further refined. However, such efforts are beyond the scope of this investigation.

With regards to the development of a highway guardrail system, various glass fiber composite materials were used. Recycled PP (polypropylene) channel and trapezoidal shapes as well as recycled ABS box shapes, sheets and belt-type strips were included in this evaluation. Tension, compression and bending tests were performed on these specimens. A virgin vinylester box section was also tested in bending for comparison purposes.

The offset block is the coupling element between the post and the rail of a guardrail system (Figure 1.1). The offset blocks manufactured during this research 
consisted of 4 modules joined together as shown in Figure 1.1. Materials used for manufacturing the offset block module consisted of recycled ABS pellets, glass fiber as reinforcement for the outer polymer shell, and rubber from tires for the inner core (Figure 1.1). Additional tests such as bond between ABS rubber surface, compression and impact on coupons from currently used wood offset block were also carried out. More information about materials and manufacturing of specimens can be found in chapters 3 and 4. A summary of tests and types of specimens is shown in Table 1.3.

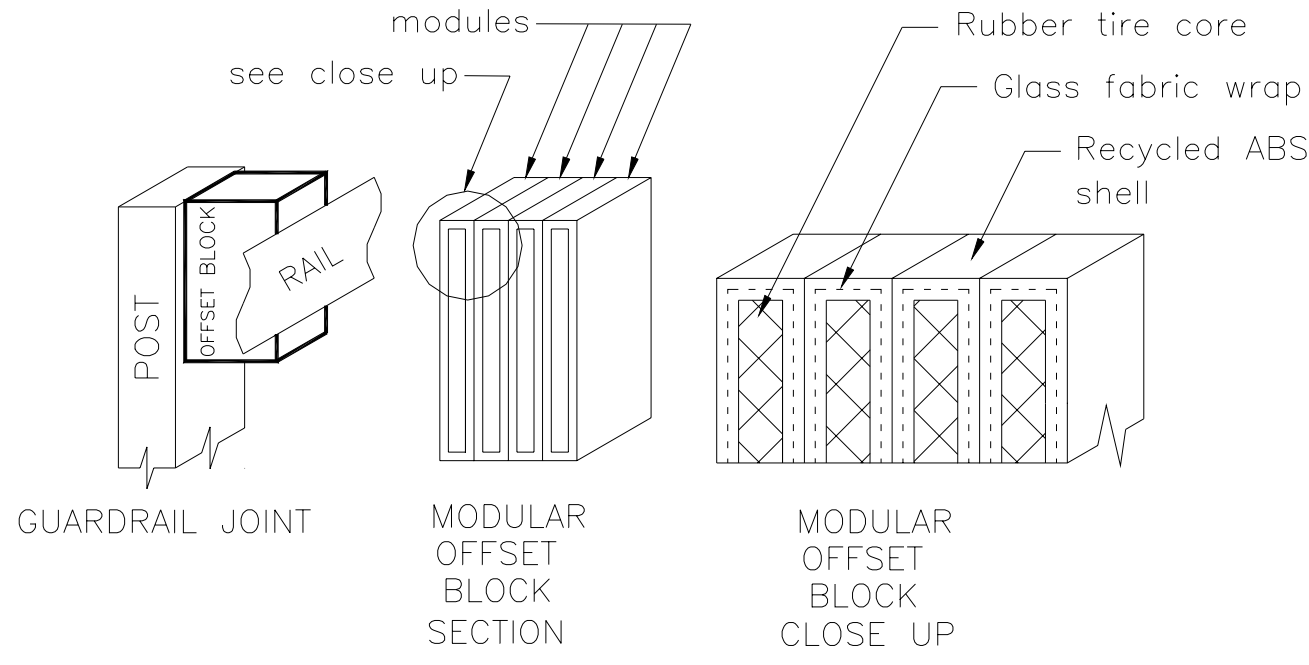

Figure 1.1 Typical Guardrail Joint Configuration and Modular Offset Block 
Table 1.3 Guardrail Post, Rail and Offset Block Specimens Tested

\begin{tabular}{|c|c|c|c|c|c|c|c|c|c|}
\hline \multirow{2}{*}{ Test } & \multirow{2}{*}{ 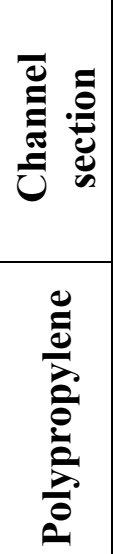 } & \multirow{2}{*}{ 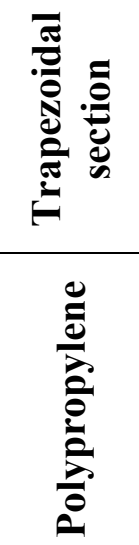 } & \multicolumn{2}{|c|}{ 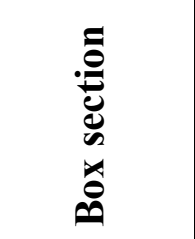 } & \multirow{2}{*}{ 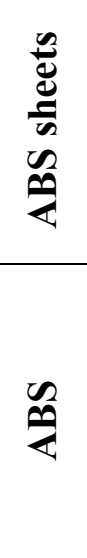 } & \multirow{2}{*}{ 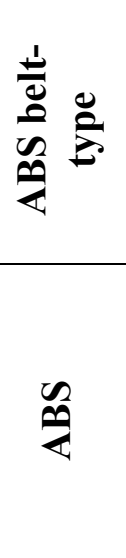 } & \multirow{2}{*}{ 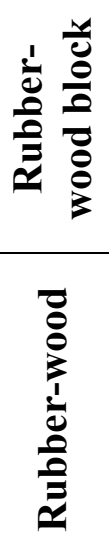 } & \multirow{2}{*}{ 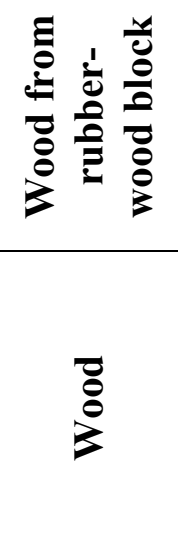 } & \multirow{2}{*}{ 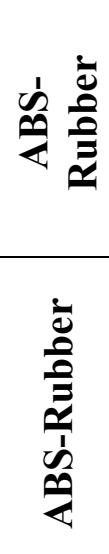 } \\
\hline & & & 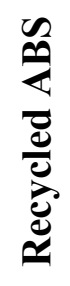 & 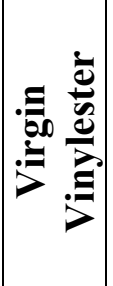 & & & & & \\
\hline Bending & 1 & 1 & 3 & 2 & 4 & - & - & - & - \\
\hline Tension & - & $\begin{array}{c}5 \\
\text { (strips) }\end{array}$ & 1 & - & 2 & 6 & - & - & - \\
\hline Compression & - & - & - & - & 2 & - & 1 & $\begin{array}{l}3(\mathrm{~Pa})^{*} \\
2(\mathrm{Pe})^{*}\end{array}$ & - \\
\hline Impact & - & - & - & - & - & - & - & 6 & - \\
\hline $\begin{array}{l}\text { Bond } \\
\text { strength }\end{array}$ & - & - & - & - & - & - & - & - & 6 \\
\hline Total & 1 & 6 & 4 & 2 & 8 & 6 & 1 & 11 & 6 \\
\hline
\end{tabular}

*Pa $=$ Parallel to grain, $\mathrm{Pe}=$ Perpendicular to grain

In Chapter 2, a summary on recycling of plastics, including processing and utilization is given. Also, examples of applications of recycled plastics in infrastructure are provided.

Chapters 3 and 4 include a detailed description of the materials as well as preparation and testing procedures of the standardized test specimens. A comprehensive description of the steps that led to manufacturing of offset block modules is provided in chapter 4 including details of recycled polymer post and rail specimens. 
Test results, calculations, analysis of data and comparison of mechanical properties and accelerated aging of specimens are discussed in chapter 5. Chapter 6 describes results and analyses for recycled polymer shapes.

Finally, Chapters 7 and 8 include a summary of the results and findings on retention of thermo-mechanical properties of aged polymer coupons and evaluation of polymer shapes for highway guardrail applications. Suitability of recycled composites for infrastructure applications is discussed and recommendations towards future research are also provided. 


\section{CHAPTER 2}

\section{LITERATURE REVIEW}

\subsection{Introduction}

Economics coupled with environmental factors of consumer products have been contributing to the growing preoccupation of people involved in industrial nations. To contain the waste, a variety of high-volume consumer products for potential recycling are: 1) soft drink bottles, 2) cans, 3) discarded appliances like computers, and 4) machinery such as heavy-duty equipment.

Experts have identified three main benefits attributable to recycling:

- Reduction in space for disposal capacity

- Lowered emissions from landfills and incinerators

- Reduction in litter and improper disposal

The three most important benefits resulting from the use of recycled materials are:

- Reduction in energy use and related emissions

- Reduction in emission of toxic gases due to reusability of polymers, and improvement in extraction and manufacturing processes

- Long-term value of conservation of raw materials (Ackerman, 1997)

Research in many areas of recycling of plastics and their applications is being conducted. Unfortunately, the development of new recycling techniques and applications has not received a major sponsorship from manufacturing industry, which would have helped making additional advances in this subject. Reasons for lack of participation of 
industry are low cost of virgin materials and limited amount of available raw materials needed for new sophisticated machines (Tall et al. 1998).

Two main topics are discussed in the following sections: 1) recycling process of plastics and 2) current utilization and practical examples of three uses of recycled plastic in infrastructure (guardrails, bridges and railroad ties). Discussion of these two topics will give an idea of what needs to be done in order to obtain the recycled plastics and what has been done in terms of recycled plastic applications.

Examples of recyclable materials and their recycle potential are given in Table 2.1 below from National Association of Recycling Industries NESS, (Barton, 1979).

Table 2.1 Types of Recyclable Materials

\begin{tabular}{|c|c|c|c|}
\hline Material & Example & Recycle Potential & $\begin{array}{c}\text { Recycle Rate, } \\
\% \text { of Potential }\end{array}$ \\
\hline $\begin{array}{l}\text { Manufacturing } \\
\text { residues }\end{array}$ & Drosses, slags, skimmings & $25-75 \%$ recoverable & Over 75 \\
\hline $\begin{array}{l}\text { Manufacturing } \\
\text { trimmings }\end{array}$ & $\begin{array}{l}\text { Machining wastes, } \\
\text { blanking and stamping } \\
\text { trimmings, casting wastes }\end{array}$ & $90 \%$ recoverable & Nearly 100 \\
\hline $\begin{array}{l}\text { Manufacturing } \\
\text { overruns }\end{array}$ & $\begin{array}{l}\text { Obsolete new parts, extra } \\
\text { parts }\end{array}$ & $\begin{array}{l}\text { Variable } \\
\text { compositions }\end{array}$ & Nearly 100 \\
\hline $\begin{array}{l}\text { Manufacturing } \\
\text { composite wastes }\end{array}$ & $\begin{array}{l}\text { Galvanized trimmings, } \\
\text { blended textile trimmings, } \\
\text { coated paper wastes }\end{array}$ & $\begin{array}{l}\text { Often not all } \\
\text { constituents } \\
\text { recovered }\end{array}$ & $0-100$ \\
\hline File dusts & $\begin{array}{l}\text { Brass mill dust, steel } \\
\text { furnace dust }\end{array}$ & $\begin{array}{l}\text { Often not economical } \\
\text { to recover }\end{array}$ & Under 25 \\
\hline Chemical wastes & $\begin{array}{l}\text { Spent plating solutions: } \\
\text { processing plant sludges, } \\
\text { residues, and sewage } \\
\end{array}$ & Often recoverable & Under 10 \\
\hline Old "pure" scrap & Cotton rags, copper tubing & $\begin{array}{l}\text { Over } 90 \% \\
\text { recoverable material }\end{array}$ & Over 75 \\
\hline $\begin{array}{l}\text { Old composite } \\
\text { scrap }\end{array}$ & $\begin{array}{l}\text { Irony die castings, auto } \\
\text { radiators, paper-based } \\
\text { laminates }\end{array}$ & $\begin{array}{l}\text { Often not economical } \\
\text { to recover valuable } \\
\text { materials }\end{array}$ & $0-100$ \\
\hline Old mixed scrap & $\begin{array}{l}\text { Auto hulks, appliances, } \\
\text { storage batteries }\end{array}$ & $\begin{array}{l}\text { Not all materials } \\
\text { recovered }\end{array}$ & Under 50 \\
\hline Solid wastes & $\begin{array}{l}\text { Municipal refuse, } \\
\text { industrial trash, } \\
\text { demolition debris }\end{array}$ & $\begin{array}{l}\text { Very low recovery } \\
\text { rates now }\end{array}$ & Under 1 \\
\hline
\end{tabular}




\subsection{Recycling Aspects}

The two principal steps on the recycling process are collection and sorting or separation.

By 1990 over 3000 curbside recycled programs existed in the USA. Campaigns promoting office and house recycling are being effective. By 1998 the number of people involved in recycling programs has risen (Powelson et al., 1992).

Many separation and sorting techniques have been developed in order to separate the collected materials (Bargo, 2000). Some existing methods include:

- Magnetic separator: Recovers ferrous material

- Eddy current device: Recovers aluminum material

- Disc screen: Separates smaller from larger material

- Trommel screen: Separates smaller from larger size particles of plastic material

- Vibrating screen: Separates material according to mesh size

- Oscillating screen: Similar to vibrating screen

- Traveling chain curtain: Separates high dense material from less dense material

- Air classifier: Separates light material from heavy material.

The amount of recyclable material collected in a certain community must meet the capacity of its separation facilities. Over collection must be avoided in order to prevent problems of recycled material storage (in small landfills). An example of an over collection problem was observed in Germany, one of the leading countries in recycling. 
Recyclable plastic waste was supplied to steel industry to serve as a reducing agent (Ackerman, 1997). This practice was economically convenient but environmentally prejudicial.

\subsection{Recycled Plastic Utilization}

Polyethylene terephthalate (PET) is one of the most extensively recycled resins. Recycling of PET beverage containers grew more than 20 times in 10 years: from 8 million pounds in 1979 to 190 million pounds in 1989 (Bennet, 1992). Potential markets for recycled PET are:

- Civil engineering: geotextile and urethane foam

- Recreational industry: skis, surfboards and sailboats

- Other industries: carpets, fence posts, fiberfill, fuel pellets, industrial paints, strapping unsaturated polyester and paintbrushes.

Recycling of High Density Polyethylene (HDPE) used in milk jugs and water jugs, increased from 58 million pounds in 1986 to 145 millions in 1989 (Bennet, 1992). Potential markets for HDPE are:

- Civil engineering: building products, curb stops, pipes, signs and trafficbarrier cones

- General industry: kitchen drain boards, milk bottle carriers, soft drink base cups

- Agricultural, recreational and gardening industries: various products

In 1989, 60 million pounds of polypropylene (PP) were recycled. They were mainly used in manufacturing of automotive battery cases (Bennet, 1992). Other uses are 
bird feeders, furniture, pails, water meter boxes, slip-sheets, bag dispensers, flowerpots, golf equipment, pallets and carpets.

In 1989, 5 million pounds of polyvinyl chloride (PVC) were recycled, amounting to $0.06 \%$ of total PVC production (Bennet, 1992). PVC is used for downspouts, fencing corrals, handrails, hose siding, landscape timbers, sewer/drain pipes, telephone cables, and many more.

Around 20 million pounds of polystyrene (PS) were recycled in 1989, representing $0.4 \%$ of total production (Bennet, 1992). Potential uses for PS are: insulation board, appliance housings and trays.

\subsection{Previous Research on Plastics in Infrastructure}

A study on Fiber Reinforced Composites (FRC) for highway safety structures was conducted on composite W-beam guardrail and recycled composite offset blocks (Dutta, 1998).

Composite W-beam guardrail: Composites included both Fiber Reinforced Polyester and composites of recycled plastics with other fibrous additives, such as sawdust. Those composites were used to manufacture the $6.1 \mathrm{ft}$ long w-beam by hand layup and vacuum bag technology. Three different thicknesses were produced and tested under bending and crash impact. Several issues were identified:

- Maximum tensile strength of 65,000 psi was obtained after producing a series of batches by trial and error, showing that this design process is more an "art than a science". 
- Expected tensile strength of 70,000 psi was not achieved by this process. However, using a commercial fabrication process like pultrusion, the tensile strength can be increased.

- Stiffness was about one third of steel's stiffness. But this was not considered as a problem, since original shape of FRP beams is effectively recovered after load application, even after fracture of specimens.

- FRP shows higher fracture initiation energy than steel. Due to the brittle nature of FRP, lower post-fracture energy absorption was observed. However, geometrical arrangement of fibers in the composite can be controlled to provide progressive crushing failure.

Conclusions of the study (Dutta, 1998) were:

- It is possible to produce FRP W-beams with a desired strength, with the same profile, but with different thickness than the AASHTO steel beam.

- Early failures in pullout tests on joints (14\% of laminate strength) showed that splicing and jointing mechanisms are a critical area of research.

- Other shapes, different from W-beams, must be considered for FRP beams, in order to identify the most efficient shape.

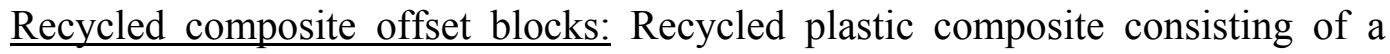
blend of 50\% sawdust and 50\% plastic waste was used to produce an offset block (Dutta, 1998). These blocks were tested under compression, tension and flexure. Several issues were identified: 
- Large compression specimens showed less variability of test results than small tension and flexure specimens.

- Moisture on the recycled composite tended to increase compressive strength and reduce flexural strength.

- At low temperatures, strain at failure decreased indicating more brittleness. Stiffness and strength decreased as well.

As a result of the research, the author concludes that relating mechanical properties of offset blocks to design of guardrail systems is not straightforward. Analytical design involves many factors including post and rail structural response, as well as soil restraining forces. Research must be carried on this topic.

In the town of New Baltimore, New York, the first recycled plastic bridge (thermoplastic) in the world was designed and built. It is a single lane bridge, $11 \mathrm{ft}$ wide with a $30 \mathrm{ft}$ span, designed for AASHTO H-15 truck. Its primary load carrying structure was made out of Fiber Reinforced Plastic Lumber (FRPL) (McLaren, et.al. 2001). Superstructure consists of two parallel bowstring trusses with transverse floor beams framing into panel points.

Design of structural members was verified with flexural and tensile tests, while bolted connections were verified with tensile tests. Monitoring program is being conducted in order to confirm design predictions.

Material used for this bridge represents the recycling of 70,000 one-gallon milk jugs. The total weight of FRPL superstructure was $11,000 \mathrm{lb}$ plus $5,400 \mathrm{lb}$ of steel connection plates. 
Another application of recycled plastics in infrastructure is railroad ties. Since 1994, Rutgers University has been developing polymer railroad ties as an alternative to wood ties (Plastics Resource, 2002). Around 700 million wooden ties are annually installed and nearly 14 million are replaced. In Altoona, PA, railroad ties made of $100 \%$ recycled high-density polyethylene (HDPE) showed a much better performance than wood ties. In addition, railroad ties installed in Pueblo, CO, with a train running 24 hours a day on a plastic railroad tie loop showed no signs of deterioration.

\subsection{Conclusions}

Recycling of plastic has been rapidly increasing in recent years. A variety of consumer applications for recycled plastics have been found. Beverage containers are believed to be one of the most widely used applications for recycled plastics. Applications in construction industry include traffic signboards, geotextiles, fence posts, and barrier cones.

Research conducted on fiber reinforced polyester (Dutta, 1998), demonstrated the suitability of plastics for use as W-beams in highway guardrail systems. Test results showed a good tensile strength $(65,000 \mathrm{psi})$. However, pullout tests showed low resistance $(8,336 \mathrm{psi})$, and may affect the splices and joints of the rails.

Compression tests as well as non-standard flexural and tensile tests were also conducted on recycled offset blocks (Dutta, 1998). Even though specimens showed satisfactory compressive strength, it was concluded that the design and development of these blocks must include laboratory testing and analysis of forces acting on the block such as rail response and post-soil interaction. 
As an example of a structural application, a recycled plastic bridge was designed and constructed (McLaren, et.al. 2001). The bridge designed for AASHTO H-15 truck is $11 \mathrm{ft}$ wide and has a $30 \mathrm{ft}$. All of the structural members were made of reinforced plastic. Another example of applications is the recycled plastic railroad ties installed in Altoona, PA. These ties showed no signs of deterioration neither on tests nor actual railroad lines.

Recycled polymers such as high density polyethylene, polypropylene, PVC and polyester are currently being recycled for infrastructure applications. Lightweight, corrosion resistance, easy transportation and installation, and durability are some of the reasons why recycled plastic products are advantageous for the construction industry.

From the three examples described, we can conclude that applications of recycled plastics in infrastructure are gradually being developed. Laboratory test and field monitoring have corroborated effectiveness of the design of structural members. In order to come up with adequate knock down factors of mechanical properties, further research has to be conducted on aging and long term evaluation of recycled thermoplastics. 


\section{CHAPTER 3}

\section{MATERIALS, PROCESS AND TEST PROCEDURE FOR CONDITIONED RECYCLED THERMOPLASTIC COUPONS}

\subsection{Introduction}

Fiber reinforced polymers (FRP) and unreinforced polymers were tested during this research to evaluate long term retention of mechanical properties and to develop structural products using these recycled polymers. ABS and PC coupon specimens evaluated for long-term retention of mechanical properties were manufactured by injection molding process.

Specimen dimensions and test procedures used for this research were in accordance with ASTM standards (Table 3.5) to evaluate mechanical properties, i.e., tension, compression, bending, impact and hardness. Different types of recycled materials used in this process are:

- Chopped glass fibers as reinforcement

- Virgin and recycled thermoplastics as matrix

The following sections include a description of the materials (polymers and glass fibers) used in this research and a summary of the manufacturing process of the coupons. Also, in this chapter, aging procedure for polymer composite specimens is described. A detailed description of tension, bending, compression, impact, hardness and creep tests are provided, including specimen geometry, preparation and procedure. 


\subsection{Materials}

$\mathrm{ABS}$ and PC resins (virgin and recycled) in the form of pellets/flakes were used to carry out the research. Chopped glass fibers were used as reinforcement to make composite test specimens for our research. Continuous fibers used to make structural shapes are described in chapter 4 .

\subsubsection{Virgin Polymers}

Based on their feasibility for commercial applications and availability, CycolacGPM5500 acrylonitrile-butadiene-styrene (ABS) with ultimate tensile strength of 7,000 psi and Lexan-101 polycarbonate (PC) with ultimate strength of 9,000 psi were chosen for manufacturing the specimens.

\subsubsection{Recycled Polymers}

Recycled polymers from discarded computers, monitors and printers in the form of pellets with a maximum diameter of about 0.25 " were supplied by MBA polymers. Purity levels of recycled polymers were above 90\%. Approach shown in Appendix I can be used to theoretically calculate the stiffness of polymers with randomly oriented chopped fibers.

\subsubsection{Glass Fibers}

Two types of glass fibers were selected based on their sizing compatibility with different resins in this research. They were manufactured for the CFC-WVU by Owens Corning (for ABS resins) and PPG (for PC resins).

Glass reinforcement compatible with ABS consisted of 4mm long 408A-14C CRATEC chopped strands with a diameter of $14 \mu$. The production process of these strands is made at a ISO 9002 register glass fiber facility, using the following steps: 
strands were chopped to a specific length from continuous glass filaments gathered together in a single bundle and then dried, screened and cleared of metallic particles.

E-glass chopped strands 3762 MaxiChop® with length varying from $3 \mathrm{~mm}$ to $13 \mathrm{~mm}$ and $13 \mu$ diameter were used for polycarbonate specimens. The production process of these strands consisted of collimating of continuous fibers into strands and either chopping directly under the bushing or winding the strands into an intermediate package for chopping in a separate operation.

\subsection{Manufacturing of Specimens}

Based on the type of fabrics, resin, melt and cure temperature, cost effectiveness and type of part, different manufacturing methods such as compression and injection molding can be used. In this research, injection molding was selected. Chopped fiber addition of $25 \%$ of total weight of the composite was selected to increase the tensile and compressive strength of some of the specimens without a considerable reduction in impact strength.

\subsubsection{Injection Molding Process}

ABS injection molding was carried out by Owens Corning Inc., using a Cincinnati Milacron injection-molding machine with a 200-ton press as described in Table 3.1. PC samples were injection molded by PPG, as described in Table 3.2. 
Table 3.1 Injection Molding Process for ABS Samples (CFC-WVU and Owens Corning Partnership)

\begin{tabular}{|l|l|}
\hline \multicolumn{1}{|c|}{ Step } & \multicolumn{1}{|c|}{ Description } \\
\hline Drying of pellets before injection process & $\begin{array}{l}\text { GPM5500 standard drying specifications } \\
\text { for general purpose: } 2-4 \text { hours drying time } \\
\text { at } 180-200^{\circ} \mathrm{F}\end{array}$ \\
\hline Melting and blending of resins & $\begin{array}{l}\text { Pellets were melted and blended together } \\
\text { at } 530 \text { to } 535^{\circ} \mathrm{F} \text { in a screw extruder with a } \\
\text { L/D ratio of } 24: 1 \text { and a compression ratio } \\
\text { of } 3.75: 1 \text { long enough to prevent non- } \\
\text { homogeneous melting and degradation or } \\
\text { discoloration (GE Plastics 1999) }\end{array}$ \\
\hline Extrusion & At 530 to $535^{\circ} \mathrm{F}$ \\
\hline
\end{tabular}

Table 3.2 Injection Molding Process for PC Samples (CFC-WVU and PPG Partnership)

\begin{tabular}{|l|l|}
\hline \multicolumn{1}{|c|}{ Step } & \multicolumn{1}{c|}{ Description } \\
\hline Drying of pellets before injection process & 5 hours drying time at $200^{\circ} \mathrm{F}$ \\
\hline Molding & $\begin{array}{l}\text { Pellets were melted at } 595^{\circ} \mathrm{F} \text { in a screw } \\
\text { with a speed of } 3.65 \mathrm{inch} / \mathrm{sec} \text { and a back } \\
\text { pressure of } 100 \mathrm{psi}\end{array}$ \\
\hline Extrusion & At $595^{\circ} \mathrm{F}$ \\
\hline
\end{tabular}

According to the type of resin (virgin, 100\% recycled or blend) and chopped glass fiber addition (reinforced or non-reinforced), six types of samples were obtained for each resin (ABS and PC) from the injection process, as shown in Tables 3.3 and 3.4. 
Table 3.3 Types of ABS Specimens

\begin{tabular}{|c|c|c|}
\hline \multirow[t]{2}{*}{ Specimens } & \multicolumn{2}{|c|}{ Description } \\
\hline & Resin & Fibers \\
\hline $\mathrm{A} 1$ & Virgin ABS polymer & Without fibers \\
\hline A2 & Virgin ABS polymer & $\begin{array}{l}\text { With } 25 \% \quad(\text { wt. } \%) \quad(12 \% \text { Fiber } \\
\text { Volume Fraction) chopped fibers }\end{array}$ \\
\hline A3 & $100 \%$ recycled ABS polymer & Without fibers \\
\hline A4 & $100 \%$ recycled ABS polymer & $\begin{array}{l}\text { With } 25 \% \quad(\text { wt. } \%) \quad(12 \% \text { Fiber } \\
\text { Volume Fraction) chopped fibers }\end{array}$ \\
\hline A5 & $\begin{array}{l}\text { Recycled ABS }(20 \%) / \text { Virgin ABS } \\
(80 \%) \text { blend }\end{array}$ & Without fibers \\
\hline A6 & $\begin{array}{l}\text { Recycled ABS }(20 \%) / \text { Virgin ABS } \\
(80 \%) \text { blend }\end{array}$ & $\begin{array}{l}\text { With } 25 \% \quad(\text { wt. } \%) \quad(12 \% \text { Fiber } \\
\text { Volume Fraction) chopped fibers }\end{array}$ \\
\hline
\end{tabular}

Table 3.4 Types of PC Specimens

\begin{tabular}{|c|c|c|}
\hline \multirow[t]{2}{*}{ Specimens } & \multicolumn{2}{|c|}{ Description } \\
\hline & Resin & Fibers \\
\hline P1 & Virgin PC polymer & Without fibers \\
\hline P2 & Virgin PC polymer & $\begin{array}{l}\text { With } 25 \% \quad(\text { wt. } \%) \quad(12 \% \text { Fiber } \\
\text { Volume Fraction) chopped fibers }\end{array}$ \\
\hline P3 & $100 \%$ recycled $\mathrm{PC}$ polymer & Without fibers \\
\hline P4 & $100 \%$ recycled PC polymer & $\begin{array}{l}\text { With } 25 \% \quad(\text { wt. } \%) \quad(12 \% \text { Fiber } \\
\text { Volume Fraction) chopped fibers }\end{array}$ \\
\hline P5 & $\begin{array}{l}\text { Recycled ABS }(20 \%) / \text { Virgin ABS } \\
(80 \%) \text { blend }\end{array}$ & Without fibers \\
\hline P6 & $\begin{array}{l}\text { Recycled ABS }(20 \%) / \text { Virgin ABS } \\
(80 \%) \text { blend }\end{array}$ & $\begin{array}{l}\text { With } 25 \% \quad(\text { wt. } \%) \quad(12 \% \text { Fiber } \\
\text { Volume Fraction) chopped fibers }\end{array}$ \\
\hline
\end{tabular}


Different shapes of coupon type samples without fibers and also with fibers (volume fraction of $12 \%$ ) were obtained and tested under tension, compression, bending impact hardness and creep. Dimensions of the specimens and test procedures according to the ASTM standards are listed in Table 3.5

Table 3.5 Coupon Dimension and Test Procedure as per ASTM Standard

\begin{tabular}{|l|l|}
\hline \multicolumn{1}{|c|}{ Test } & \multicolumn{1}{c|}{ Standard } \\
\hline Tension & ASTM D638-94b \\
\hline Compression & ASTM D695-91 \\
\hline Bending & ASTM D790-92 \\
\hline Impact & ASTM D256-93 ${ }^{\mathrm{a}}$ \\
\hline Hardness & ASTM D2240-91 \\
\hline Creep & $\begin{array}{l}\text { Sustained load, modified tension coupons } \\
\text { were used. }\end{array}$ \\
\hline
\end{tabular}

\subsection{Aging (conditioning)}

In order to evaluate the long term mechanical property retention of the recycled materials, specimens were subjected to an accelerated aging process in a salt environment.

Samples were kept immersed in plastic containers consisting of a 3\% $\mathrm{NaCl}$ (by weight) solution, placed inside an environmental chamber that runs the temperature cycle shown in Figure 3.1, throughout the year. Samples were removed for testing at 2, 4, 10 and 18 months of aging. Salt solution was selected because of its use as a deicing agent for roads and highways during winter periods. It was found that one day of aging in 3\% salt solution at an average temperature of $34^{\circ} \mathrm{F}$ is equivalent to 17 days of weathering at normal environmental conditions (Vijay et al., 1999). 
The study on creep behavior does not include aged samples. Creep tests started in February 2000 (Bargo, 2000) and data have been recorded since then.

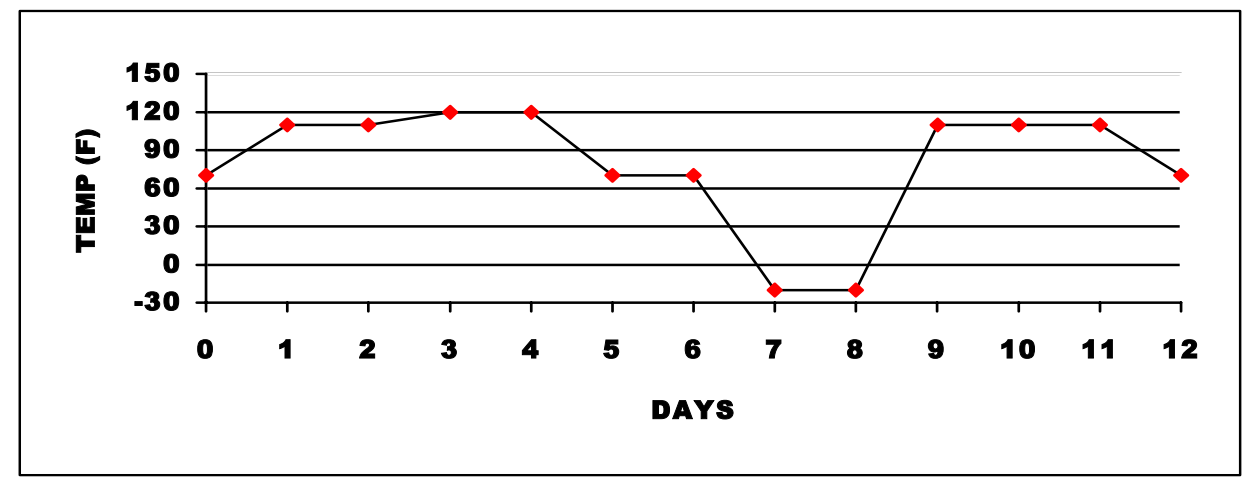

Figure 3.1 Conditioning (Aging) Temperature Cycle

\subsection{Testing}

In the following sections, a description of the tests carried out is given, which includes specimen, specimen preparation, test set up and procedure.

\subsubsection{Tension Test}

\subsubsection{Test Specimen}

For both ABS and PC, tension coupons tested were "dog-bone" shaped, as shown in Figure 3.2. Dimensions selected according to ASTM D638-94b for Type I specimen were as follows:

Width of narrow selection (W)

$0.5^{\prime \prime}$

Length of narrow section (L)

$3.0 "$

Width overall (WO)

$0.75 \%$

Length overall (LO)

$8.5^{\prime \prime}$

Gage length $(\mathrm{G})$

$1.0 "$

Distance between grips (D)

$4.5^{\prime \prime}$

Thickness (T)

$0.125 ”$ 


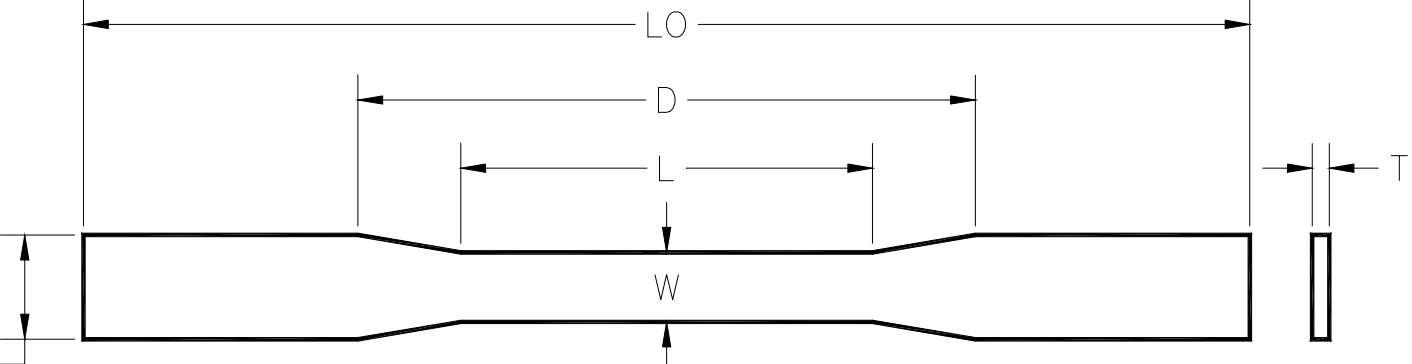

Figure 3.2 Tension Specimen Dimensions

Samples were labeled according to the type of test conducted, type of specimen tested and specimen number. For instance sample TA1-1 was a tension specimen (T), virgin ABS without fibers (A1, refer to Table 3.3), and specimen number was 1.

\subsubsection{Specimen Preparation}

In order to measure tensile strains on each tested sample, a series 2620 dynamic extensometer with a gage length of 1.00 inch was attached at the midsection of each sample as shown in Figure 3.3.

\subsubsection{Test Set-up and Procedure}

Test set-up and an Instron 8500 two-column load frame-testing machine are shown in Figures 3.3 and 3.4. The test was completely computer controlled, including the data recording of strain, deflection and testing time. "Wavemaker " software provided by the loading frame manufacturer was utilized to carry out the tests. Specimens were loaded to failure at a constant rate of $300 \mathrm{lb} / \mathrm{min}$. 


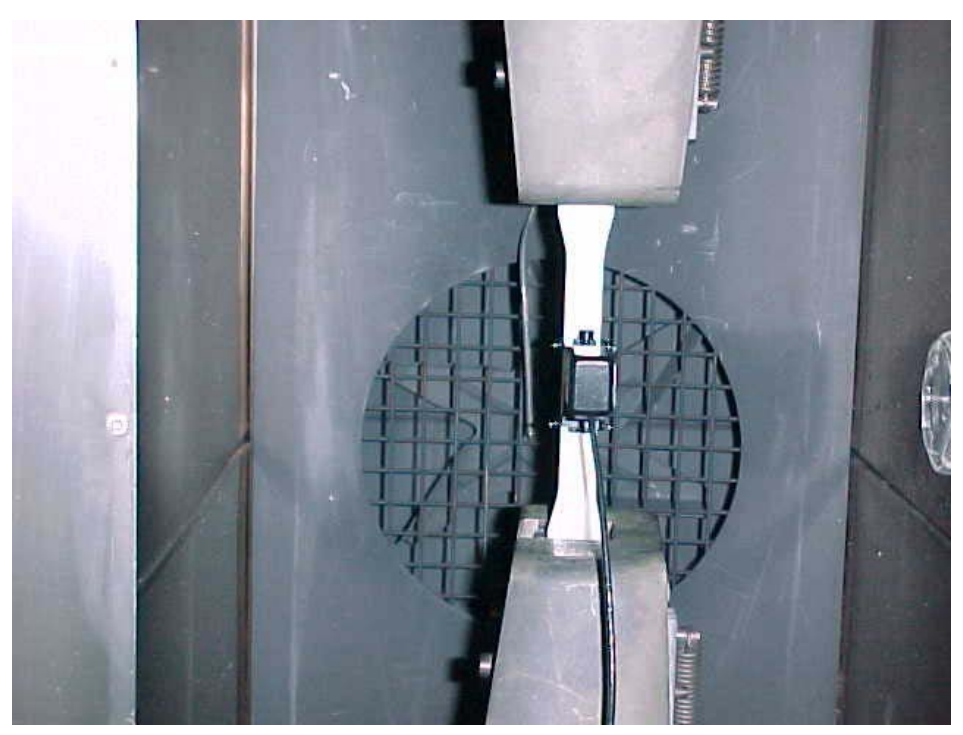

Figure 3.3 Close-up of Tension Test Set-up with Extensometer Attached

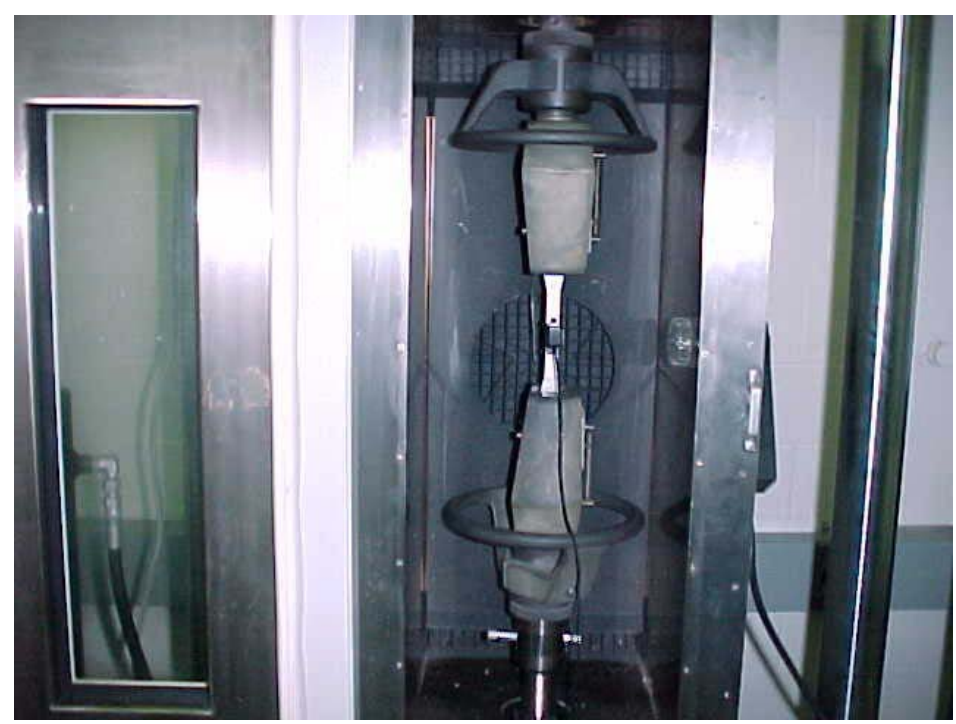

Figure 3.4 Test Set-up for Tension Test

\subsubsection{Bending Test}

\subsubsection{Test Specimen}

Rectangular specimens according to ASTM D790-92 test were manufactured for $\mathrm{ABS}$ and PC. Coupons tested under three-point bending were 5" long, 0.5" wide and $0.125 "$ thick. Samples were labeled according to the type of test conducted, type of specimen tested and specimen number. For instance sample BA1-2 was a bending 
specimen (B), virgin ABS without fibers (A1, refer to Table 3.3), and specimen number was 2. A span of 3.75" was used for bending test to match ASTM requirements of span length and overhanging length.

\subsubsection{Specimen Preparation}

A strain gage was installed on the tension side at midspan on each specimen. Surface preparation consisted of: 1) sanding with a 320 grit sandpaper, 2) cleaning with an acid surface cleaner (degreaser), 3) cleaning with water-based alkaline solutions (conditioner and neutralizer) to remove oil residues and promote adhesion, 4) attaching the gage to the specimen using M-Bond 200 adhesive followed by overnight curing with proper clamping pressure to ensure adequate bond.

\subsubsection{Test Set-up and Procedure}

An Instron 8500 two-column load frame-testing machine was used. Test setup and machine are shown in Figures 3.5 and 3.6. The test was computer controlled with the exception of recording strain data. Strain data were taken with the help of a strain indicator unit. "Wavemaker " software was utilized to conduct the tests. Specimens were loaded at a constant rate of $0.25 \mathrm{in} / \mathrm{min}$ to failure or until deflections greater than one third of the span were attained. 


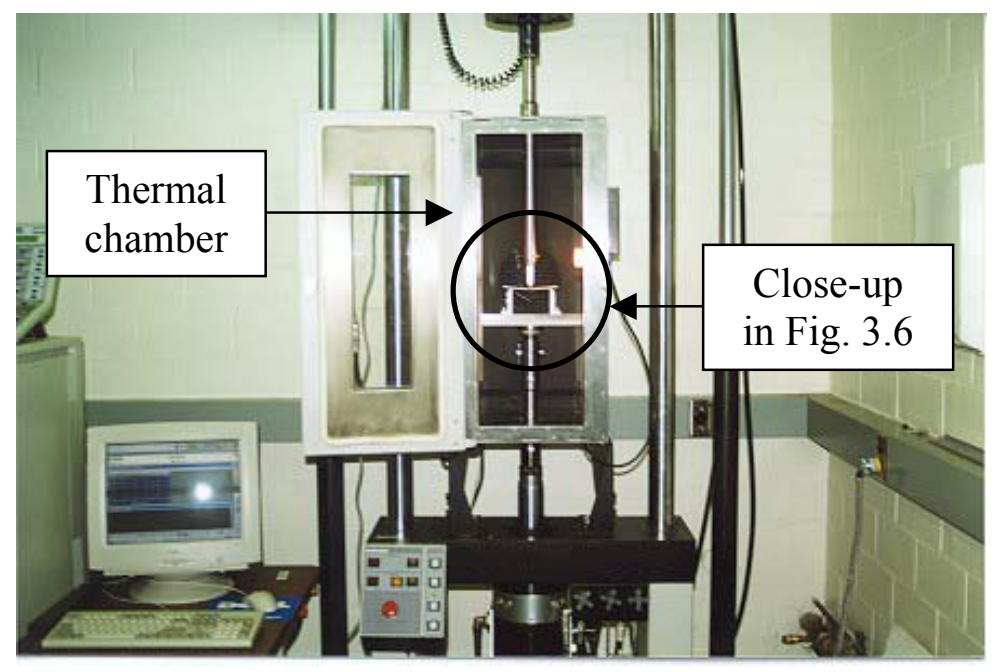

Figure 3.5 Test Set-up for Bending Test

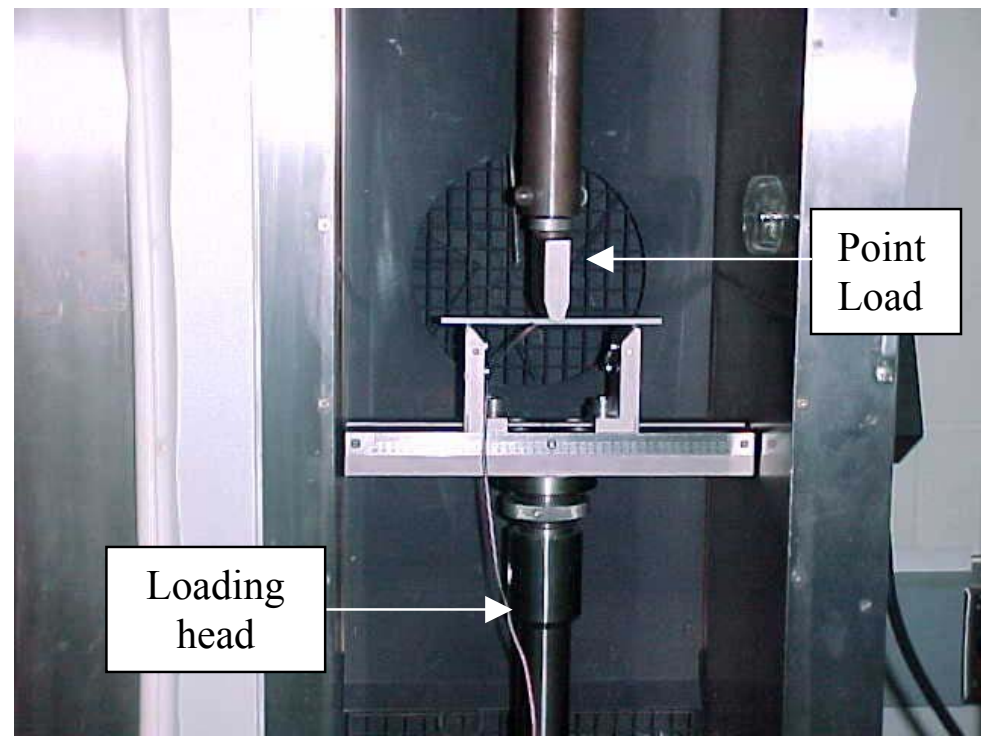

Figure 3.6 Close-up of Bending Test

\subsubsection{Compression Test}

\subsubsection{Test Specimen}

Rectangular specimens with cross section of $0.5^{\prime \prime} \times 0.25 "$, height of 0.5 ", and aspect ratio of 2 were selected as per ASTM D695-91 for both ABS and PC specimens. Samples were labeled according to the type of test conducted, type of specimen tested, and number of the specimen. For instance, sample CA1-3 was a compression specimen (C), virgin ABS without fibers (A1, refer to Table 3.3), and specimen number was 3. 


\subsubsection{Specimen Preparation}

A strain gage on one of the wider faces was installed on each specimen. Surface preparation followed the same steps as described before for bending specimens.

\subsubsection{Test Set-up and Procedure}

Test set-up and an Instron 8500 two-column load frame-testing machine are shown in Figures 3.7 and 3.8. The test was computer controlled with the exception of strain data, which was recorded with the help of a strain indicator unit. Specimens were loaded at a constant rate of $0.05 \mathrm{in} / \mathrm{min}$ to failure.

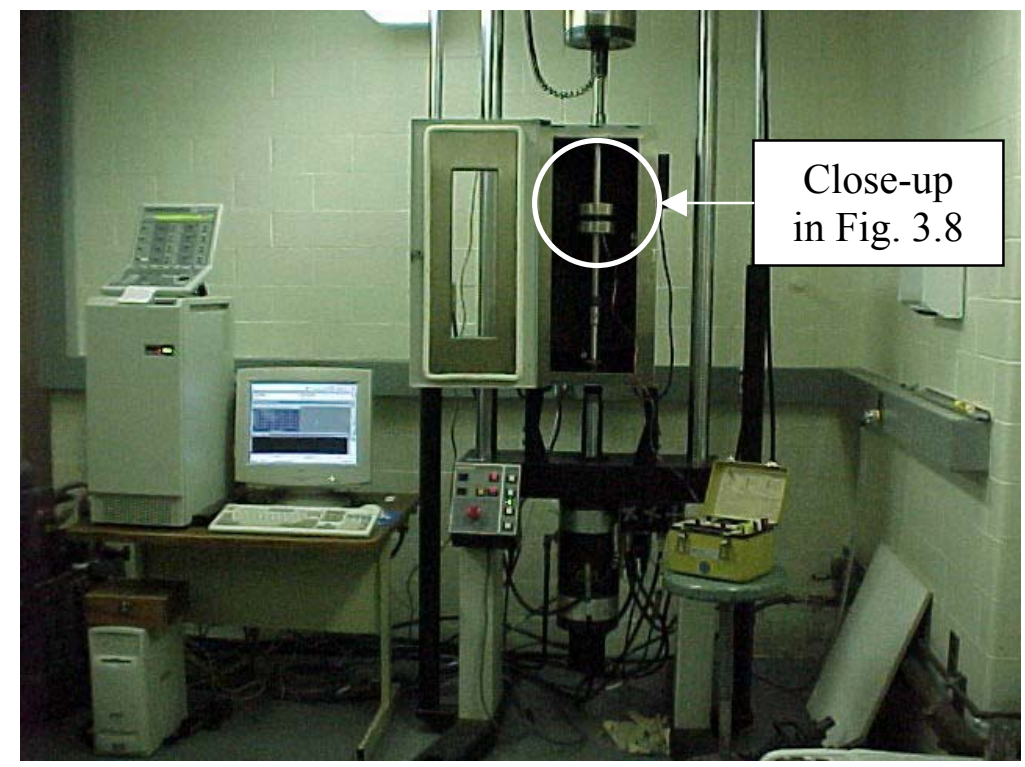

Figure 3.7 Test Set-up for Compression Test - Instron Machine 


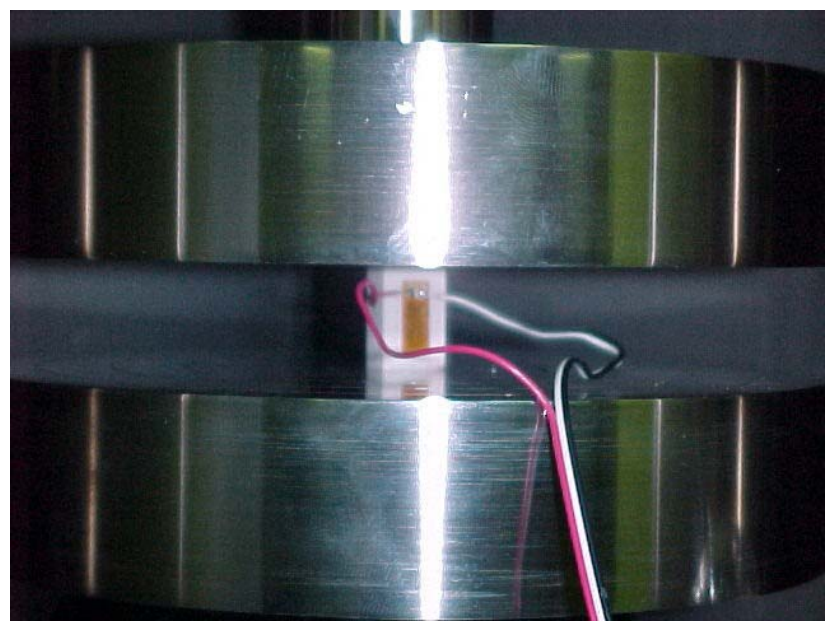

Figure 3.8 Close up for Compression Test

\subsubsection{Impact Test}

\subsubsection{Test Specimen}

Izod type test were carried out, specimen dimensions suitable for this type of test were selected according to ASTM D25-93a, as follows:

Width of notched section (A)

0.4085

Notch location (B)

$1.25 "$

Overall length $(\mathrm{C})$

$2.5 \%$

Depth of notch (D)

$0.25 \mathrm{R}$

Overall width (E)

$0.5 \%$

Thickness (F)

$0.125^{\prime \prime}$

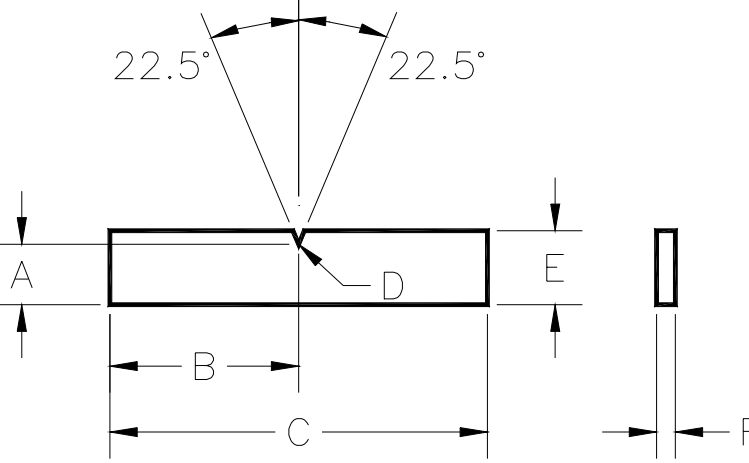

Figure 3.9 Impact Specimen Dimensions 
Samples were labeled according to the type of test conducted, type of specimen tested and specimen number. For instance, sample IA1-4 was an impact specimen (I), virgin ABS without fibers (A1, refer to table 3.3), and specimen number was 4.

\subsubsection{Specimen Preparation}

Notching of specimens was performed by Owens Corning following ASTM specifications. No further preparation of the sample was required.

\subsubsection{Test Set-up and Procedure}

A BLI Series Impact Testing Machine was used for Izod type testing of PC and ABS specimens. No additional weight was attached to the pendulum. Test set-up is shown in Figures 3.10. After pendulum was released, impact energy was directly recorded from the machine's scale. Failure was noted by observation of the remaining portion of the specimen as hinge $(\mathrm{H})$, complete $(\mathrm{C})$ or partial $(\mathrm{P})$. Readings were adjusted for pendulum friction and windage, using the standard correction chart supplied by the manufacturer (BLI Series Manual, 1999).

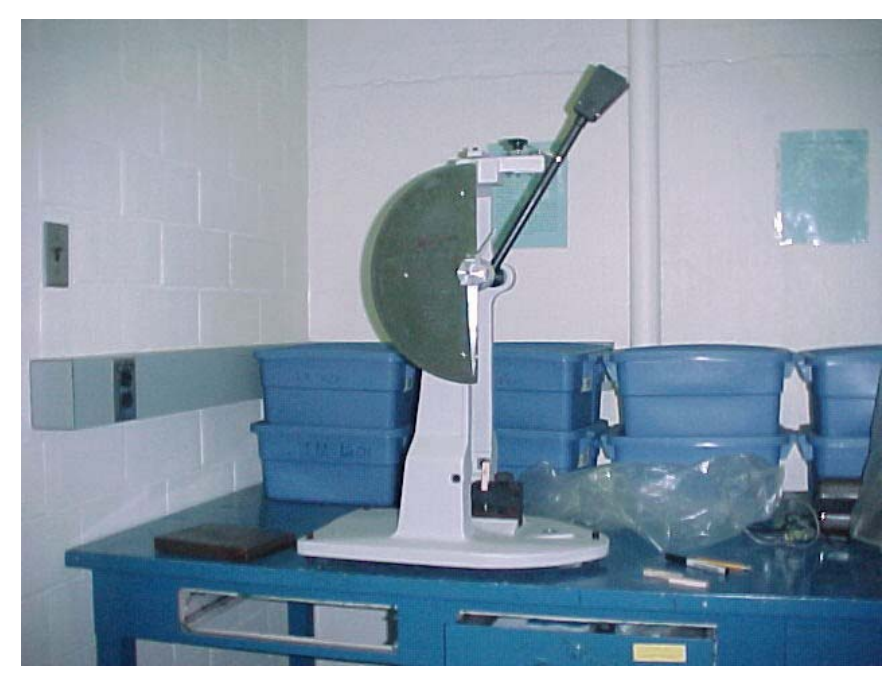

Figure 3.10 Test Set-up for Impact Test 


\subsubsection{Hardness Test}

\subsubsection{Test Specimen}

Circular specimens meeting ASTM D2240-91 for dimensions and manufacturing were used. Diameters of ABS and PC specimens were 2" and 3" respectively, and both types of specimen thicknesses were $0.125 "$. Samples were labeled according to the type of test conducted, type of specimen tested and number of the specimen. For instance, sample HA1-5 was a hardness specimen $(\mathrm{H})$, virgin ABS without fibers (A1, refer to Table 3.3), and its number was 5.

\subsubsection{Specimen Preparation}

No preparation of the sample was required for this test.

\subsubsection{Test Set-up and Procedure}

A Duotronic Model 2000 Hardness Testing Machine was used for the hardness test as shown in Figure 3.11. For all PC and ABS specimens no extra weight was added to the hammer. Series of five hardness index readings at different locations on the surface of each specimen were directly recorded from the digital display on the machine.
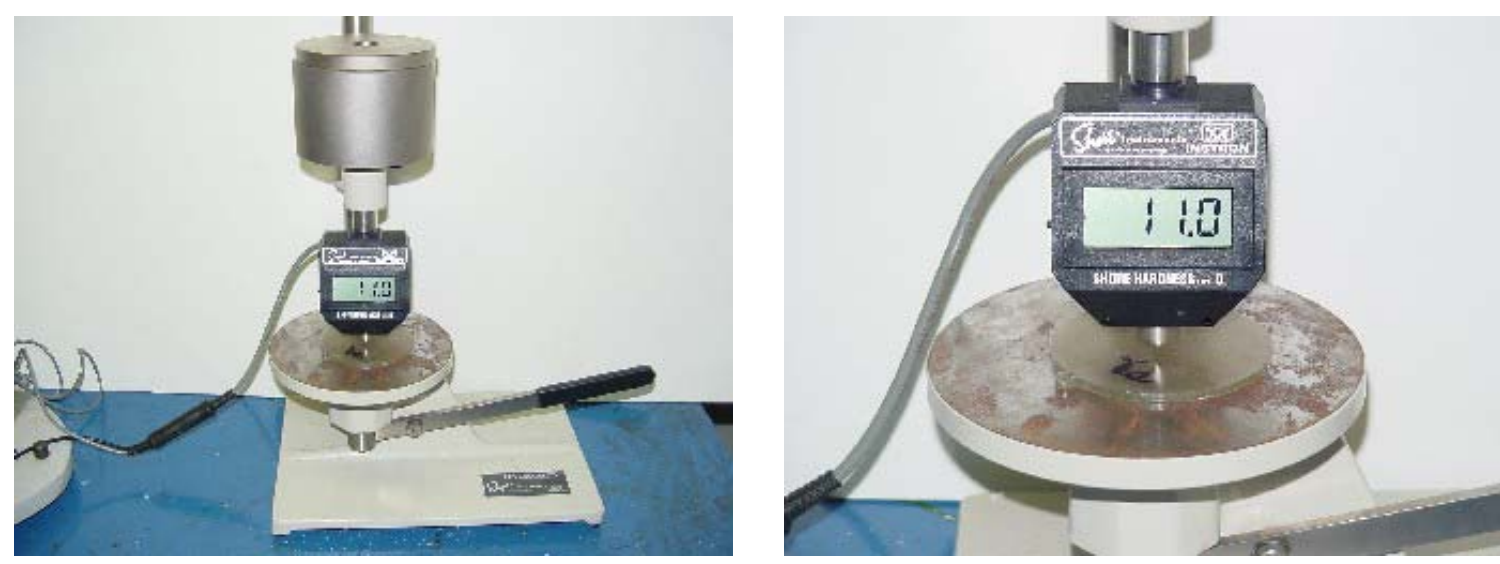

Figure 3.11 Test Set-up for Hardness Test 


\subsubsection{Creep Test}

In order to determine the deformability of reinforced and unreinforced resins (composites) under constant state of stress, creep tests were initiated by the CFC-WVU and continued under this research.

\subsubsection{Test Specimen}

Dog-bone shaped tension specimens were selected to carry out creep tests. Dimensions used were as described in section 3.5.1.1 and Figure 3.2.

\subsubsection{Specimen Preparation}

In order to apply constant tensile force to the specimens, $3 / 8^{\prime \prime}$ diameter holes were drilled on each of the grips on the samples at $1.125 "$ from the end and $0.375^{\prime \prime}$ from the sides as shown in Figure 3.12. Strain gages were attached at midlength of the specimens using the procedure described in section 3.5.2.2. Samples were suspended from the flange of a steel beam with a threaded bolt (0.375" diameter x 1.5 " length) tightened in a threaded hole in the flange of the steel beam as shown in Figures 3.13 and 3.14. Bolts were passed through supporting steel plates on both sides of the grip and through the hole drilled on the specimen. Steel plates with a known weight were hung to provide the constant dead load at the other end of the specimen. Strain readings were recorded using a strain indicator unit. 


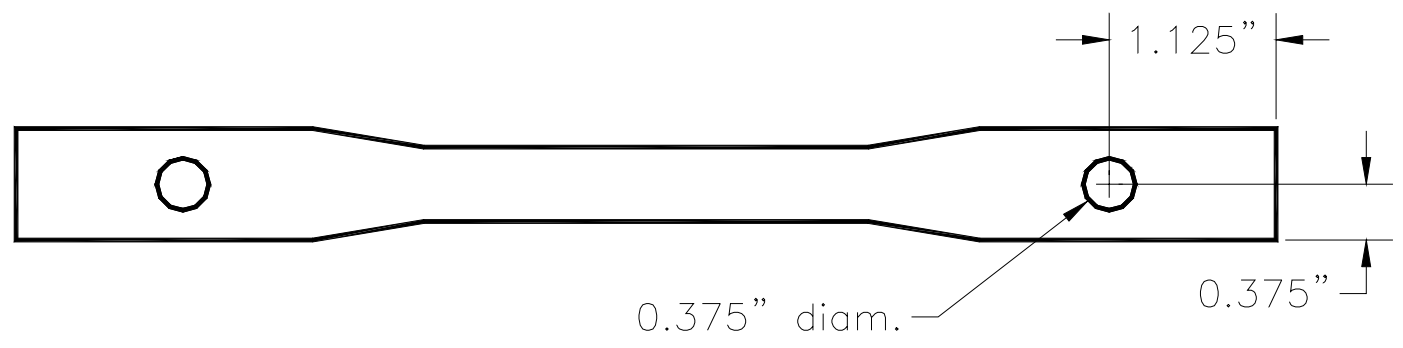

Figure 3.12 Creep Specimen
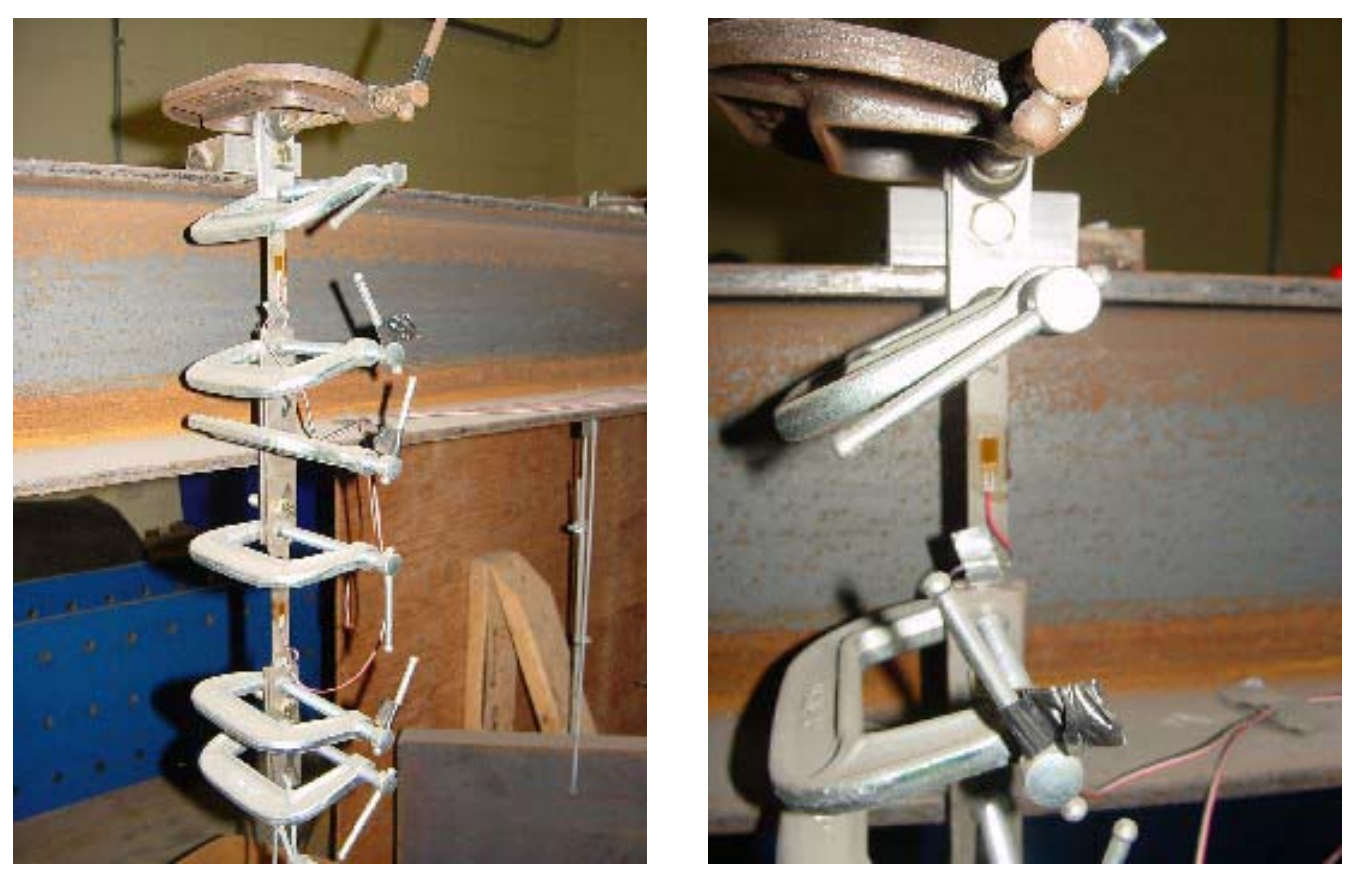

Figure 3.13 Creep Specimen Set-up 

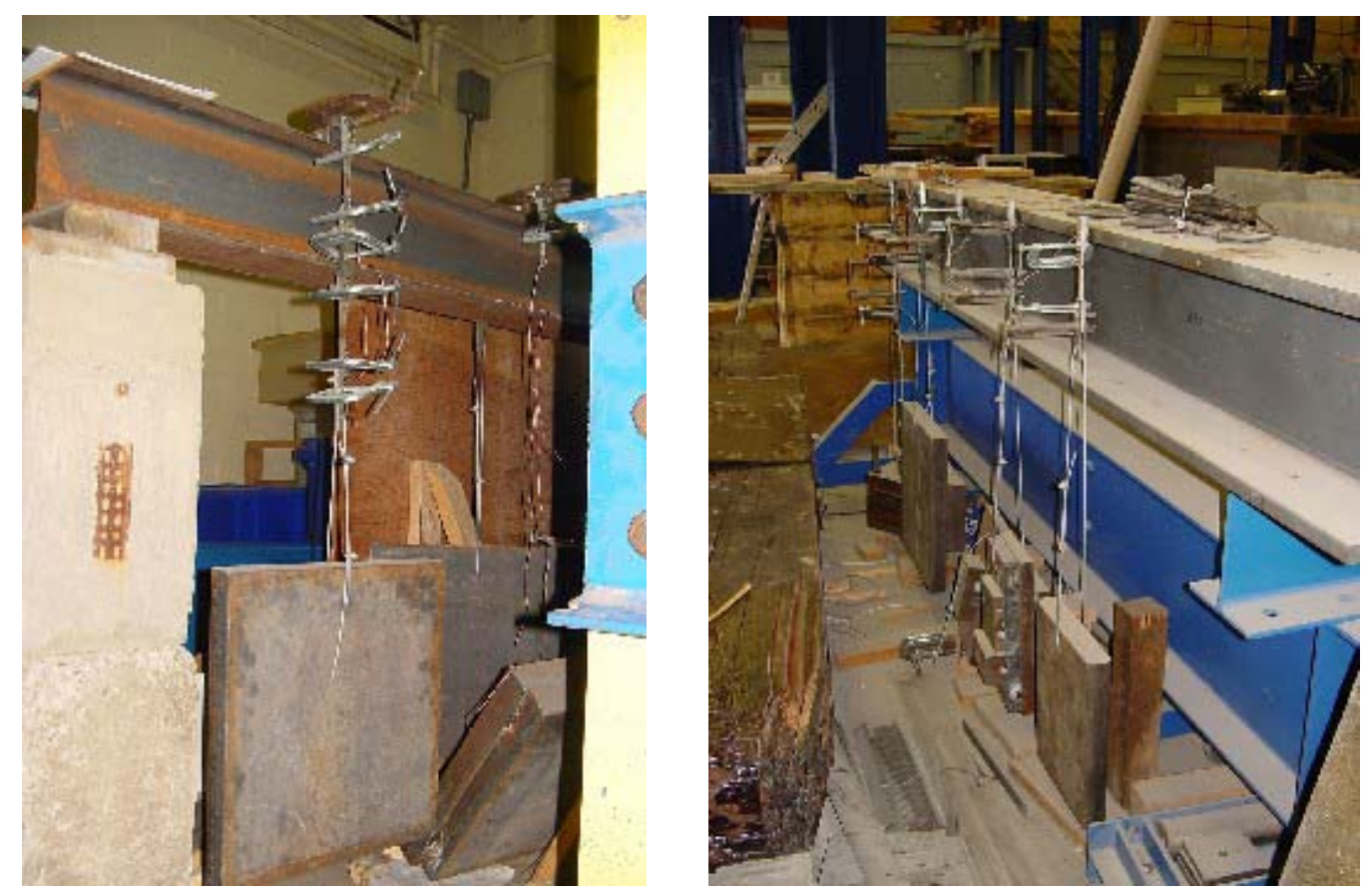

Figure 3.14 Creep Test Set-up

\subsubsection{Test Set-up and Procedure}

Strain readings were periodically recorded from a strain indicator unit connected to the strain gage. Creep coefficient for a given day of testing was calculated

$$
C c=\frac{\varepsilon t}{\varepsilon_{0}}
$$

as:

Where,

$\mathrm{Cc}=$ Creep coefficient after $\mathrm{t}$ days of loading

$\varepsilon_{\mathrm{t}}=$ Strain after $\mathrm{t}$ days of loading

$\varepsilon_{0}=$ Strain at the instance of loading 


\section{CHAPTER 4}

\section{MATERIALS, MANUFACTURING AND TEST PROCEDURE FOR GUARDRAIL POST, RAIL AND OFFSET BLOCK}

\subsection{Introduction}

Recycled fiber reinforced polymers (FRP composites) were used as main materials during this research to develop an offset block, which is a structural product for highway guardrails. Different types of materials used for the offset block are:

- Recycled thermoplastic pellets as binding material

- Glass fiber as reinforcement for the offset block

- Rubber from automobile tires as a core to absorb the vehicular impact

Post and rail shapes tested in this study were manufactured by TPI Inc, Oklahoma in collaboration with CFC-WVU. Offset blocks were manufactured at CFC-WVU, using a compression molding process.

The following section includes a description of the materials used to manufacture the offset block as well as the polymer shapes, including polymers, glass fibers and rubber tires.

A detailed description of the steps that led to manufacturing of the offset block is provided in section 4.3 , including

- Description of the compression molding procedure

- Manufacturing of the small blocks and modifications under compression molding process

- Discussion of heat transfer aspects

- Manufacturing of full size offset block modules 
Bending, tension and compression test results on polymer channel, trapezoidal and box sections are summarized and analyzed in section 4.4. Comparisons between shapes are also provided. Additional test results of bond between ABS and rubber and wood for offset blocks are also presented in this section.

\subsection{Materials}

In addition to thermoplastic resins and glass fibers used for manufacturing the components of the guardrail system (post, offset block and rail), rubber from discarded tires was used as a shock absorber and filler for the offset block.

\subsubsection{Recycled Polymers}

Two types of resins were used for the specimens tested for guardrail and post: Polypropylene specimens with trapezoidal and channel section and ABS specimens with box cross sections. In addition to these, ABS sheets were also tested at room temperature to identify the material behavior and quantify mechanical properties. All of the above specimens were pultruded using recycled thermoplastics by TPI Inc, Oklahoma and shipped to CFC-WVU before testing.

For the offset block, recycled ABS pellets with a maximum diameter of $1 / 2$ " were used as shown in Figure 4.1 ESR pellets were made available from MBA Polymer Inc, with a purity level above $90 \%$. 


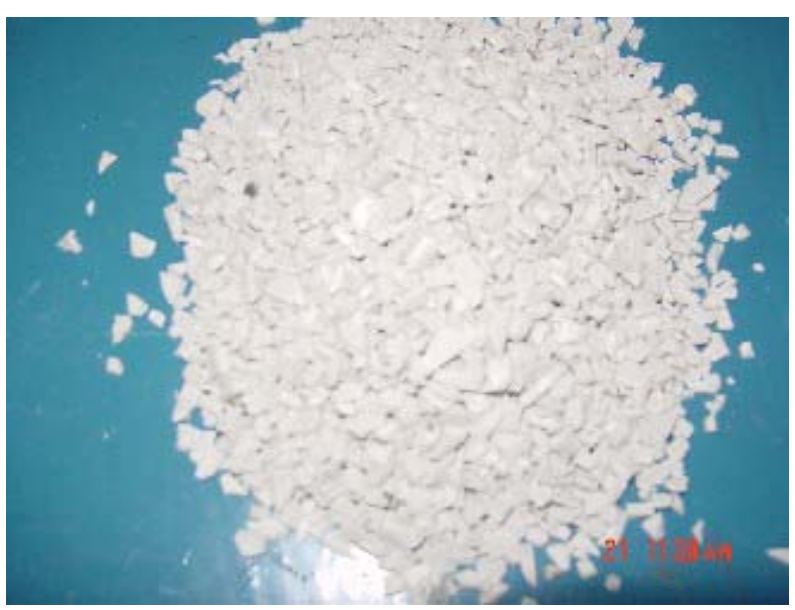

Figure 4.1 ABS Recycled Pellets for Offset Block Manufacturing

\subsubsection{Glass Fibers}

For the offset block, several types of glass fabrics were used in the form of a continuous wrap inside the block itself.

\subsubsection{Rubber Tires}

To take advantage of the shock absorption property of rubber materials, discarded rubber tires were used to manufacture offset blocks. They were obtained from automobile and tire shops and cut to convenient shape and size, with a reciprocating saw or a high speed vertical saw, according to dimensional requirements. Art of steel belted tires was perfected by trial and error during this research.

\subsection{Manufacturing of Offset Block}

Compression molding process was selected for manufacturing the offset block for the guardrail system, based on availability of the equipment and suitability of glass fabric usage as reinforcement. A PHI molding machine model SO-230H with a maximum molding force of 30 ton was used. The molding machine consists of two hot platens (top and bottom) that apply pressure to a mold placed in between them. Machine and mold schematic is shown in Figure 4.2. 


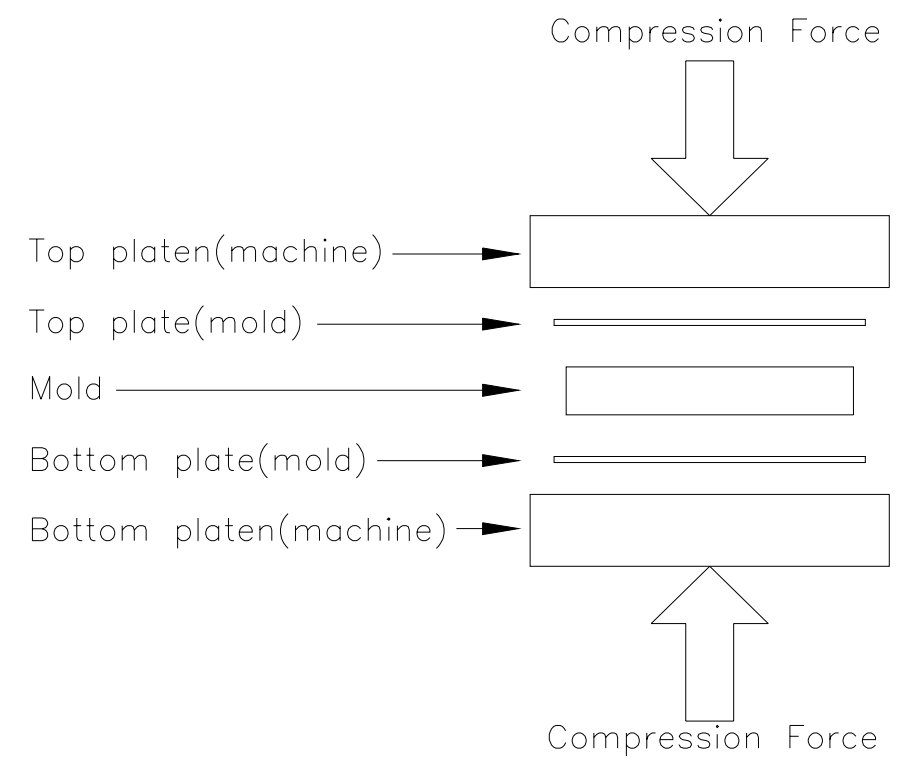

Figure 4.2 Schematic of Compression Molding

\subsubsection{Molding Procedure}

Different approaches were tried during this study in order to get an adequate homogeneity of the resin, adhesion of the fabric and final finish. The molds used in this study were made of steel and consisted of a closed rectangular box having opened bottom and top surfaces where the pellets were placed. Two pressure plates at the top and bottom surfaces close the mold and transfer the load applied by the hot platens of the molding machine. The main steps of a pressure molding process are:

- Hot platen set up: By means of a digital selector, hot plates are heated up to the desired molding temperature for the thermoplastic. In our case ABS was molded at $400^{\circ} \mathrm{F}$ and this process required about 40 minutes.

- Mold set-up: Top and bottom plates were wrapped with aluminum foil or a similar wrap for easy cleaning of the plates after molding. During the molding stage, some of the resin dripped on the plates and adhered to 
them, making it difficult to clean. Mold and plates can be oiled to de-mold the pressure-molded product after cooling.

- Positioning of pellets in the mold: Mold was placed over the bottom plate. Fillers such as wooden or rubber blocks and fabrics were placed in position inside the mold and pellets were poured into the mold filling as many voids as possible. Depending upon the desired final density of the molded product, the pellets will stand out of the mold, forming a pile. This pile will be forced into the mold during the pressure application stage (Figure 4.3).

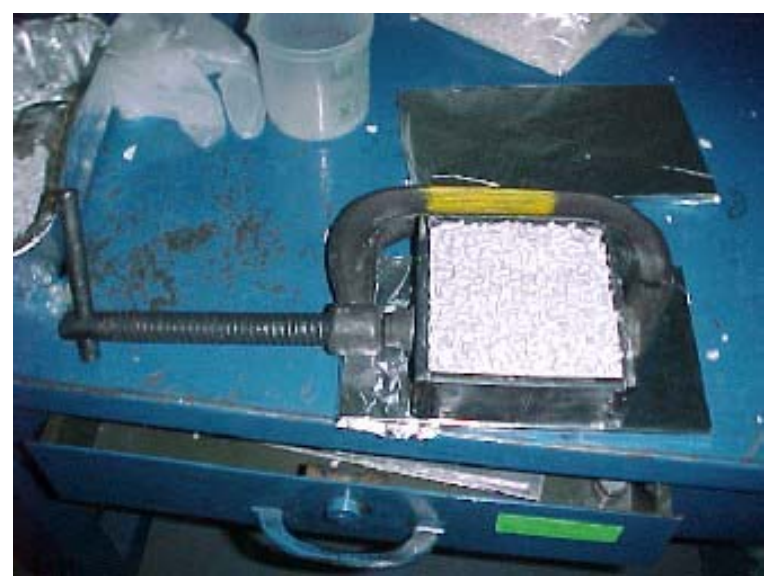

\section{Figure 4.3 Mold and Pellets for Pressure Molding}

- Molding set-up: Top plate was placed over the pellet pile and aligned with the bottom plate. Mold and plates were placed and centered over the bottom hot platen (Figure 4.4). Pumping the machine jack with the lever brings hot platens together. Molding clock was set to the selected molding time, which varied depending upon the thickness of the mold. 


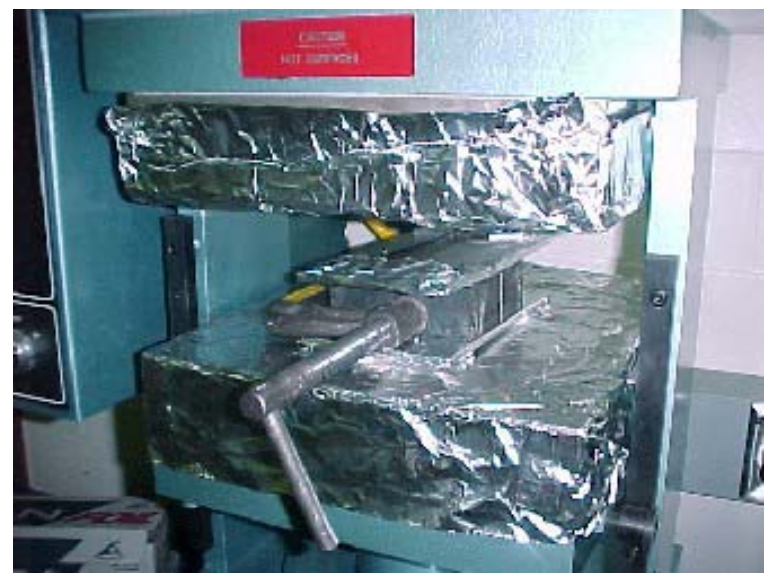

Figure 4.4 Molding Set-up

- Pressure molding: Desired pressure was applied with the hydraulic pump, in our case, i.e. 20 Tons. The pressure was held constant during the molding time. Usually it took a few minutes for the pressure to stabilize due to air flowing out of the mold. In order to maintain the pressure constant, the hydraulic pump had to be activated until the pressure reached a steady state.

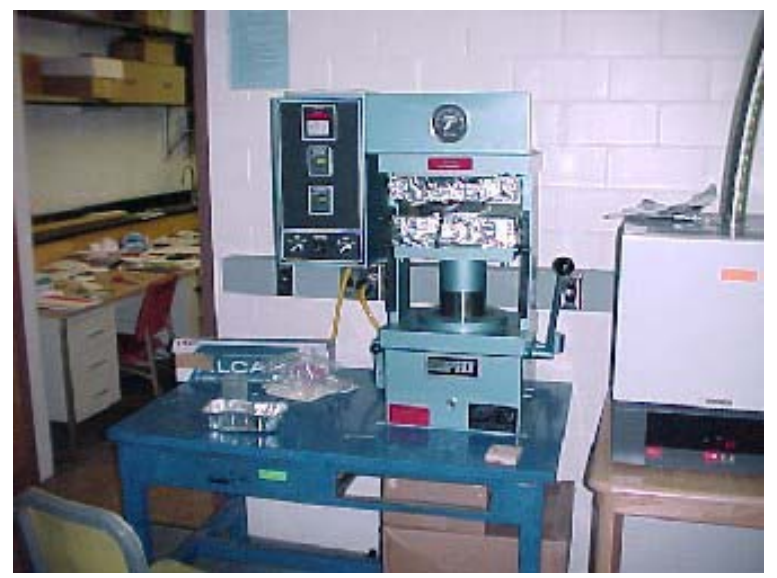

Figure 4.5 Pressure Molding Machine During Molding Process

- Cool down and de-mold: Once the molding time was over, pressure was released and mold was pulled out of the machine and placed in an isolated space for cooling. Cooling time was usually 20 to 25 minutes. A fan was 
used to reduce the cooling time, in our case to 15 minutes. Once the mold and product cooled down, top and bottom plates were removed. Excess resin on the edges of the mold was cleaned and the molded product was extracted by disassembling the mold or by hammering the final product out in the case of one-piece molds.

\subsubsection{Small Block Molding}

Small blocks were molded with and without core (rubber and wood), and also with and without fabric, to get a feel on the pressure molding process, manufacturing issues and compatibility of the resins with the core material (rubber and wood) and fabric.

Specimens and dimensions are described next:

- Solid recycled ABS small blocks: Neither fillers nor fabric were used in this block, measuring 3" x 3" and 0.5" thick. About 20 of these small blocks were produced with varying molding times and molding pressures in order to determine the optimum combination. Times varied between 2 and 5 minutes and pressures between 15 and 20 Ton. Finally, it was established that the optimum molding configuration for these small blocks was 4 minutes under 20 Ton pressure over the 3" x 3" area. Optimum parameters were determined in terms of homogeneity of the molded resin and final finish of the product. Small block specimens are shown in Figure 4.6. 


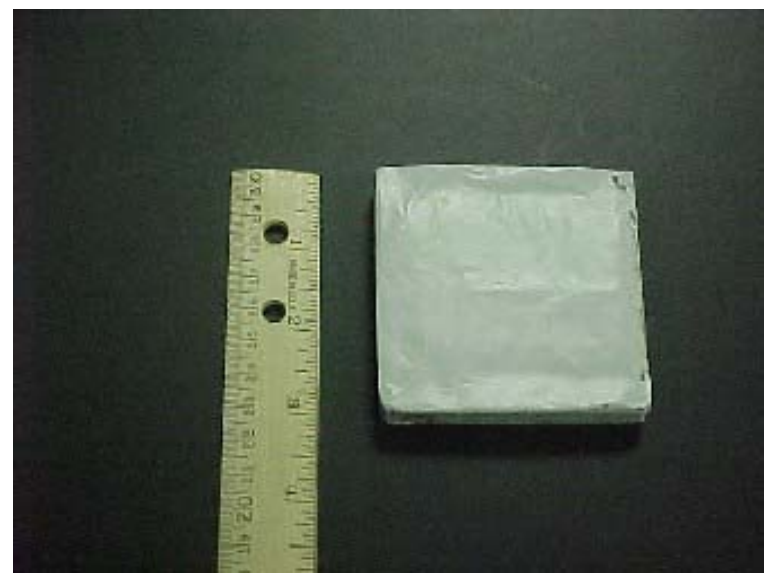

Figure 4.6 Solid Recycled ABS Small Block

- Filled recycled ABS small blocks: Next step consisted of using a filler material inside the ABS block. Filler material consisted of neoprene, wooden and discarded rubber strips. In addition, glass fabric was also used to improve properties of the block. Primer was used to improve bond between rubber and glass fabric. A number of pull-out bond tests between rubber and ABS interface were conducted as described in section 4.4.6. Dimensions of the blocks were 3" x 3.5" with a thickness of 1". Specimens are shown in Figure 4.7

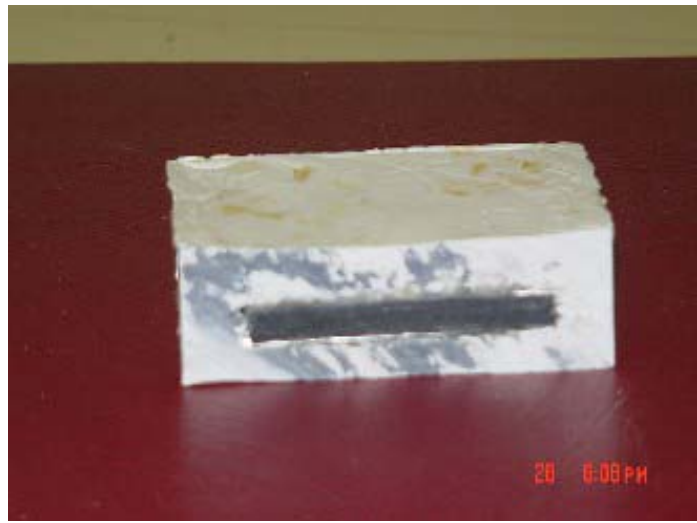

a) Neoprene Core

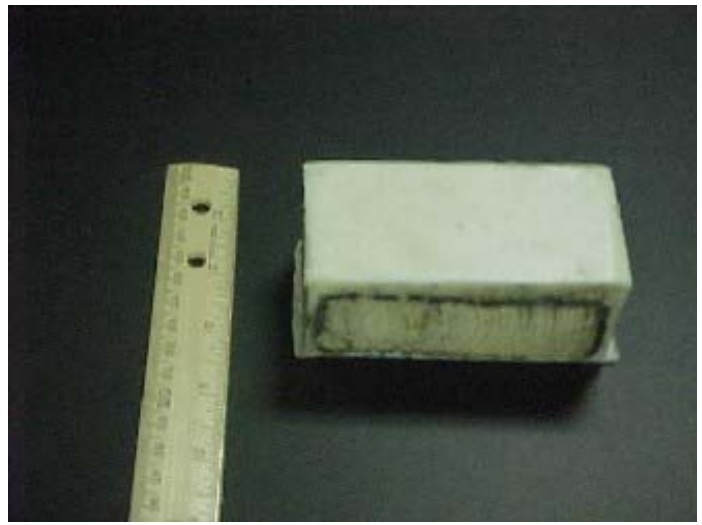

b) Wood Core 


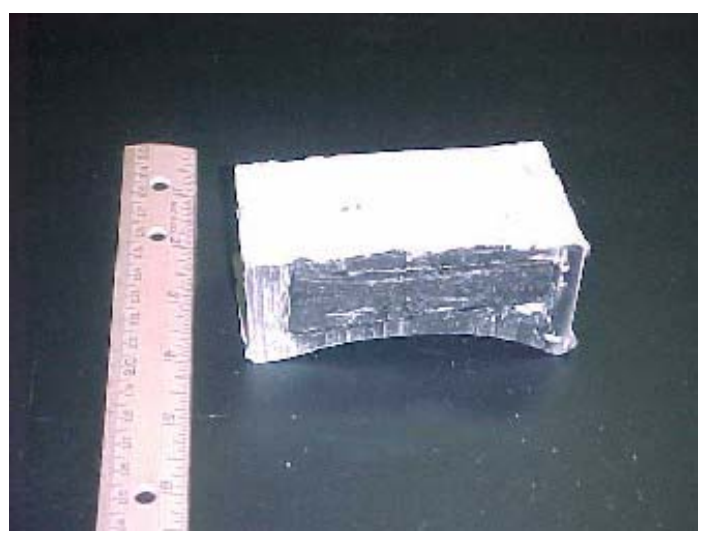

c) Rubber tire Core

Figure 4.7 Filled Small Blocks

- Two stage molded small blocks: In order to properly embed the glass fabric in the resin and facilitate the positioning of pellets between the rubber core and the fabric, a two stage molding process was used as described below:

1. An inner small block 3" x 3" x 1" with uneven rubber core and resin shell was molded to obtain a regular shape.

2. Small block described in step 1 was wrapped with glass fabric. This step minimized the voids inside the specimens. The wrapped small block was then used as filler for the final product manufacturing.

3. The filler described above was molded with ABS pellets in a bigger mold to obtain the final product. This final product was 4 " $\mathrm{x}$ 4" and 1.5" thickness. The process is shown in Figure 4.8. 
The two stage procedure was very important in this study because we found out that the heat transfer to the resin inside the wrap was not adequate during the second stage molding.

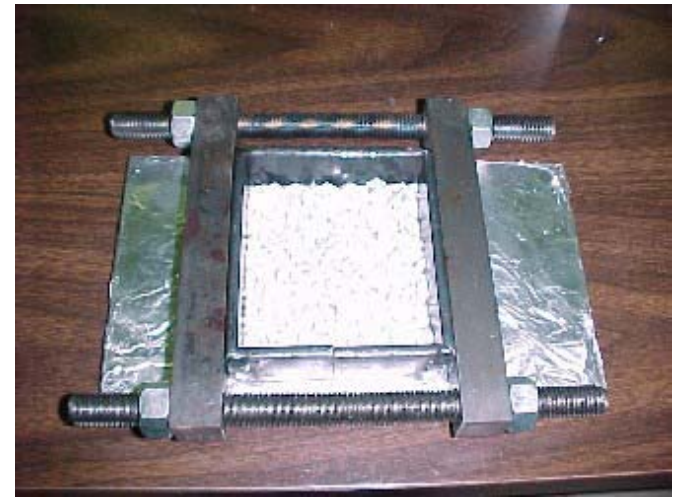

a) ABS Pellets For First Stage

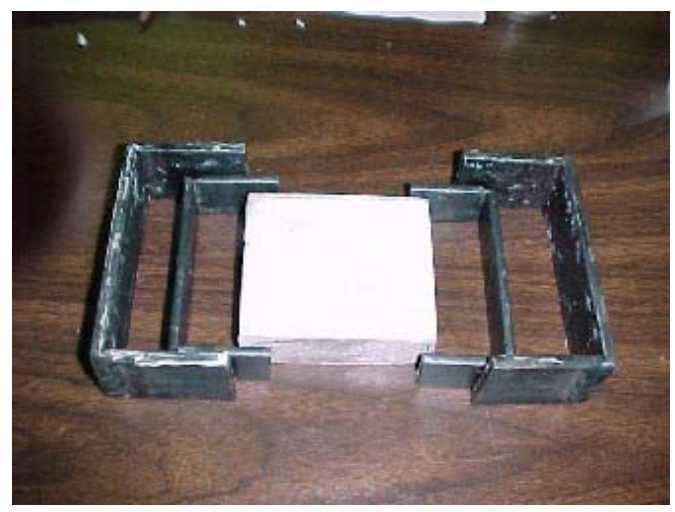

c) First Stage Inner Small Block and Molds

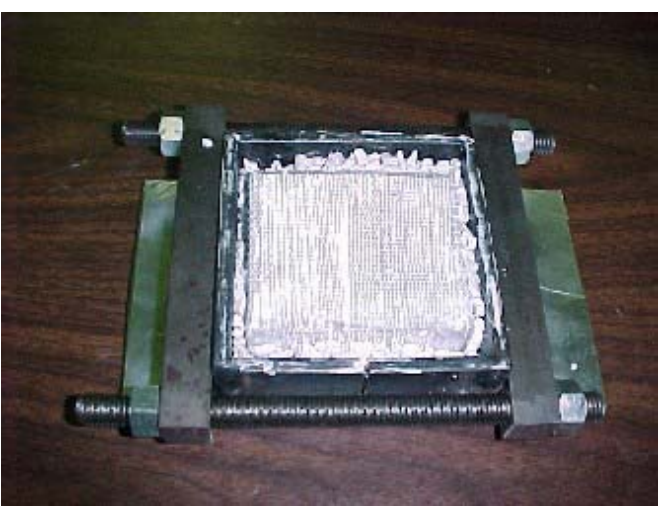

e) ABS Pellets for Second Stage

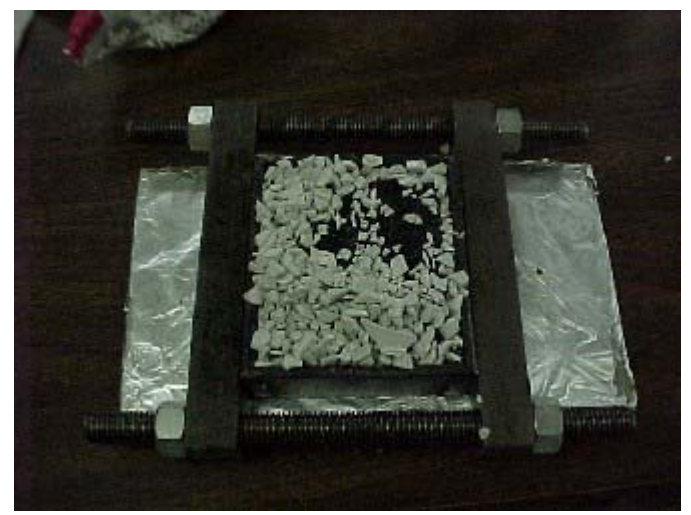

b) Rubber Core Partially Covered With ABS Pellets

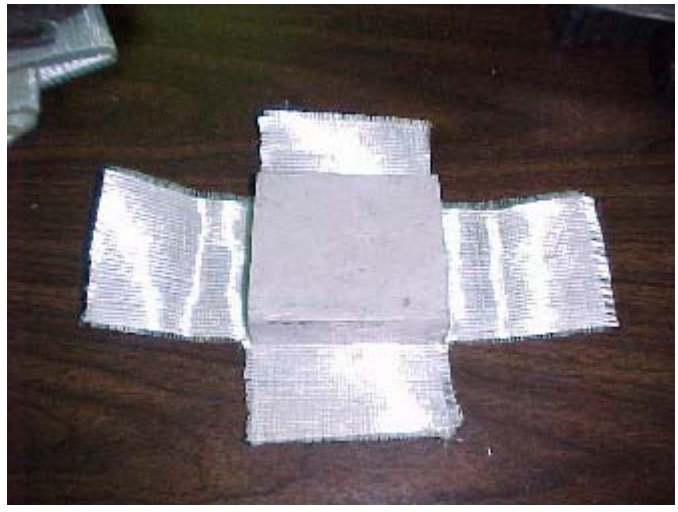

d) Fabric Wrapping of the Inner Small Block

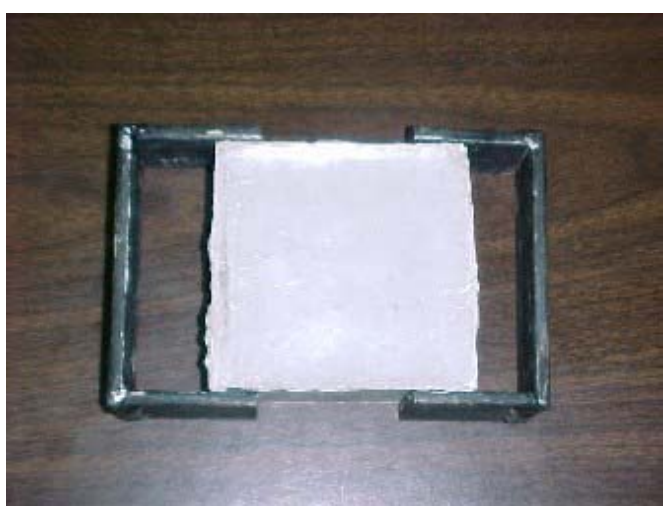

f) Final Two Stage Small Block Molding

Figure 4.8 Two Stage Molding Process 
- Rubber-Wood Block: An additional block made out of three rubber tire strips assembled between two wooden blocks with slots to hold the strips in place was also developed and tested. When tested under compression, this test specimen showed good compression strength but excessive deformation. This model demonstrated the need for a solid wrapping shell to prevent buckling of the core elements. Overall dimensions of the assembled block were 91/2" × 6" × 83/4". Dimensions of wooden blocks were $91 / 2$ " x 6 " x 2"(thick) and slots were 1" deep. Dimensions of rubber strips were approximately $81 \frac{1}{2} " \times 7 " \times 3 / 4 "$ (thick). Block is shown in Figure 4.9 .

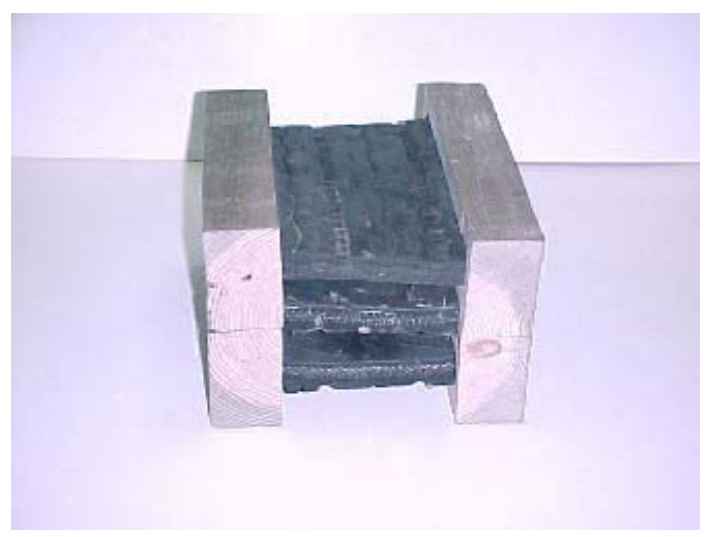

Figure 4.9 Rubber-Wood Model

\subsubsection{Heat Transfer Aspects}

One of the major concerns while compression molding an offset block was heat transfer along the depth of the member. Temperature measurements using thermocouples attached to the sides, top, and bottom plates of the steel molds revealed that the entire mold heated up to its uniform temperature in less than a minute. However, it was evident that inner pellets along the depth of the block were not heated up to thermoplastic melting temperatures. Two possibilities were considered: 
1- Increase in molding time, which led to over heating of the outside pellets causing the resin to lift up the upper plate and resin overflow during the release of the mold.

2- Pre heating of the mold with pellets, rubber and fabric properly positioned in the mold using an oven at the desired mold temperature and proceed with the molding stage with the whole product already at melting temperature. It is important to note that due to its high viscosity and low coefficient of thermal expansion, $\mathrm{ABS}$ at melting temperature does not easily flow without pressure application. Hence, after pre-heating, pellets become soft and sticky making it easier for them to bind together. This second approach was later refined and led us to a successful process.

\subsubsection{Final Product - Small Scale}

Same 4" x 4" mold with 1.5" thickness described in section 4.3.2 and used for the second stage of the two stage molding process was used to obtain small scale final product. Several runs were done following the pre-heating approach. In order to reduce voids and improve fabric wettability, virgin ABS tabs obtained from injection molding process (with a thickness of $0.125 "$ ) were cut to dimensions needed to cover the rubber core in all directions. These tabs were placed on to the rubber core using instant glue to hold them in place and to facilitate wrapping as shown in Fig. 4.10. 


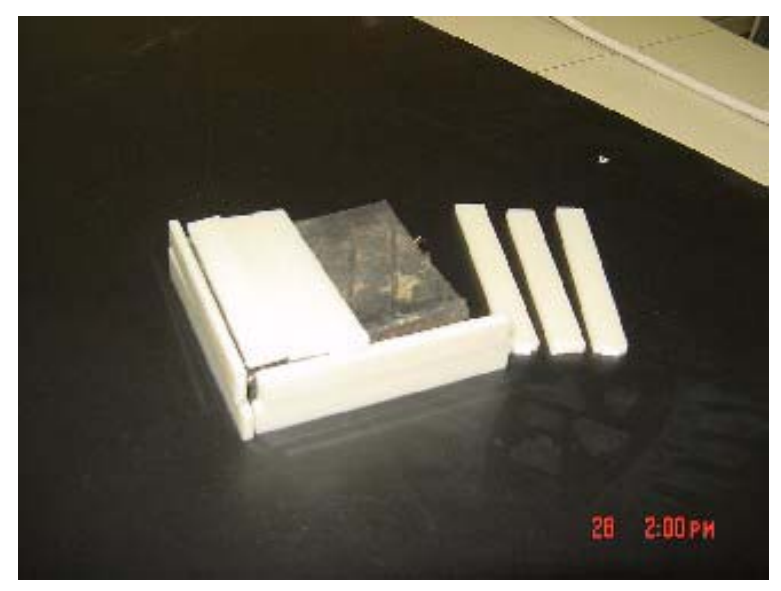

\section{Figure 4.10 Pre-molded ABS Tabs and Rubber Core}

Once the rubber core with tabs was assembled, it was placed in the mold and gaps between the core and the sides of the mold were filled with recycled ABS pellets. Additional pellets were heaped up on the top surface to provide sufficient amount of resin to fill the mold. Following items were noted:

- It is very important to provide enough pellets; otherwise during melting under pressure, pellets will not have sufficient volume to fuse together and produce a solid block. An example of insufficient resin amount is shown in Fig. 4.11. Calculation of ABS weight that can be packed in must be done to provide sufficient amount of pellets. For our ABS, we took the maximum density of packed pellets to be $67 \mathrm{pcf}$.

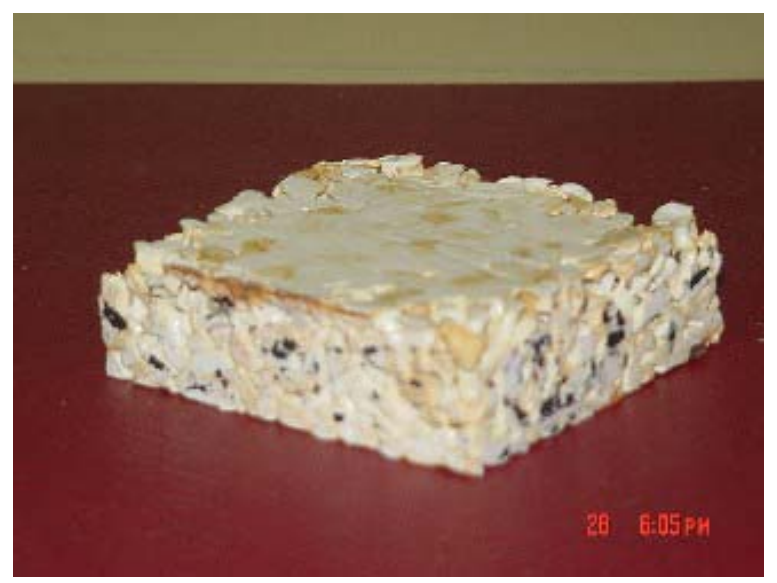

Figure 4.11 Specimen Molded With Inadequate Amount of Pellets and Solidified After Melting Under Pressure 
After placing pellets, the filled mold with top and bottom plates were placed in an oven at a temperature of $400^{\circ} \mathrm{F}$ for 15 minutes of preheating. Following preheating, the mold was placed in the compression molding machine, and 20 Tons of pressure was applied for 6 minutes. Figure 4.12 shows a cross section of one of the manufactured blocks, where pellets were totally melted together with good fiber-resin adhesion.

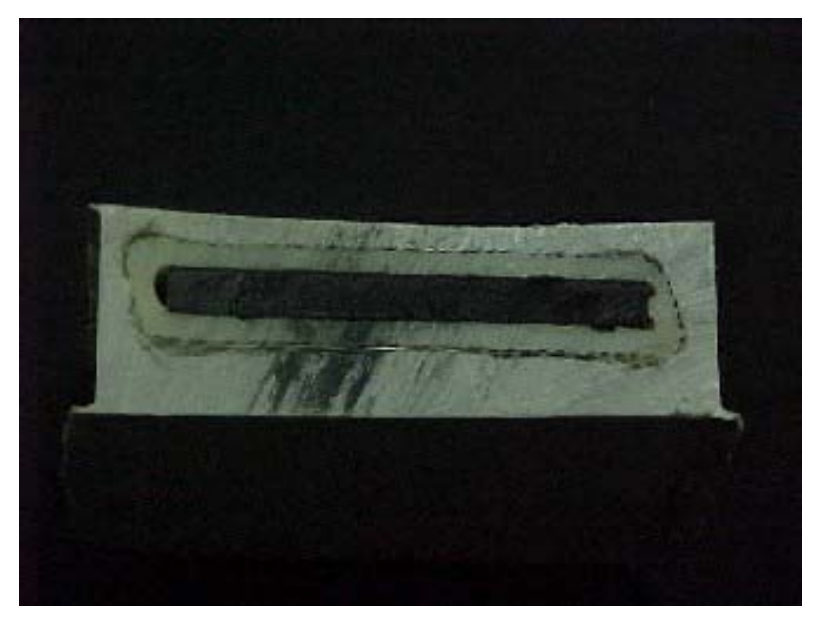

Figure 4.12 Pre-heated Molded Block

\subsubsection{Final Product - Prototype}

Schematic of standard dimension offset blocks being used in highways is shown in Figure 4.13. Manufactured offset blocks conformed to those dimensions.

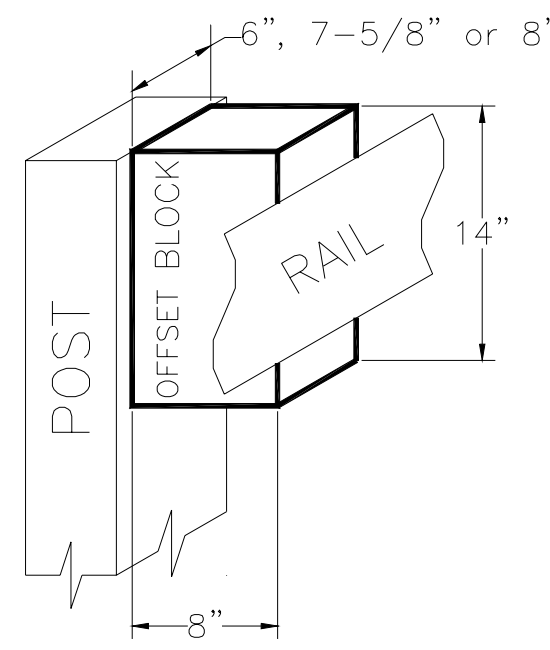

Figure 4.13 Offset Block Dimensions 
While molding the offset block, two drawbacks were noted. Hot platen dimensions were $12.5^{\prime \prime} \times 12.5 "$ and maximum opening between hot platens was 5". Obviously, two of the dimensions were smaller than the required $14 "$ x 8 " x $(6 "$ or $75 / 8$ " or $8 ")$. To overcome this problem following approach was used

1. The offset block was divided into four $14 "$ x $8 "$ × 2" modules, to get a final product (14" x 8" x 8" standard dimension for highway use). These blocks will be interlocked with bolts passing through holes drilled after molding the element.

2. The hot platen length was 1.5 " shorter than the desired final product. By rotating the mold $180^{\circ}$, a second preheating and molding cycle would be carried out on the molded product in order to provide adequate molding pressure to the $1.5^{\prime \prime}\left(14 " \mathrm{x} 12.5^{\mathrm{N}}\right.$ available in machine) portion projected out of the hot platens during the first molding phase.

For mass production of offset block, proper molding of the module in a single step can be accomplished using larger hot platens. Figure 4.14 shows the final design for the modular offset block

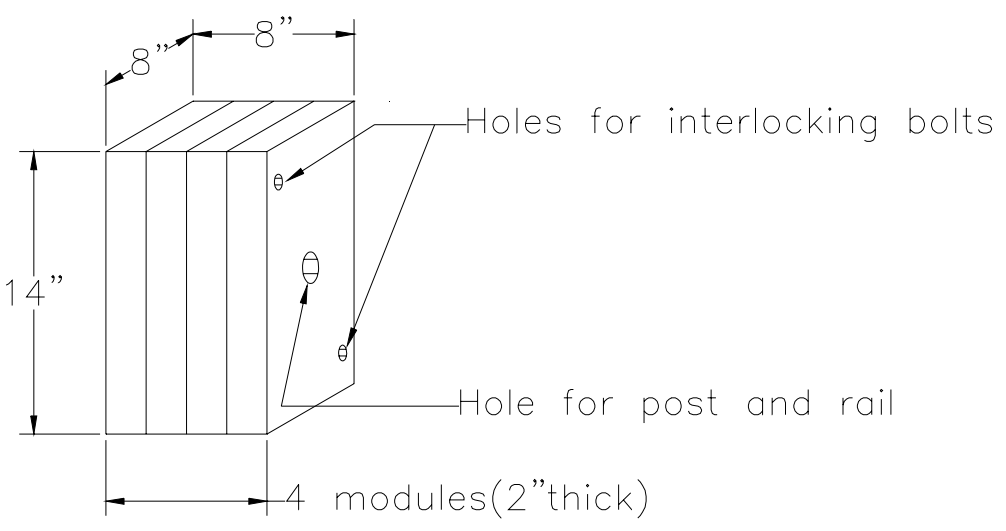

Figure 4.14 Modular Offset Block 


\subsubsection{Final Molding Procedure}

In this section, final molding procedure for module is described. Two main molding stages were identified: molding of tabs and molding of module. Molds used in this research were manufactured at CFC-WVU laboratories.

\subsubsection{Molding of Tabs}

Tab mold preparing: The mold for manufacturing tabs consisted of a $9^{1 / 4 "} \mathrm{x}$ 10 " and $1 / 4$ " thick steel plate, with three slots machined in it: two for the side tabs $\left(6 \frac{3}{4} " \mathrm{x}\right.$ $1 "$ and 7" $x$ 1") and the other one for the bottom and top tabs (63/4" x 71/2"). Top and bottom steel plates were 101/4" x 11" and 1/4" thick. Mold was cleaned and top and bottom plates were wrapped with aluminum foil or covered with a sheet of aluminum flashing (which gives a better finish). Figure 4.15 shows the mold preparation for tabs.

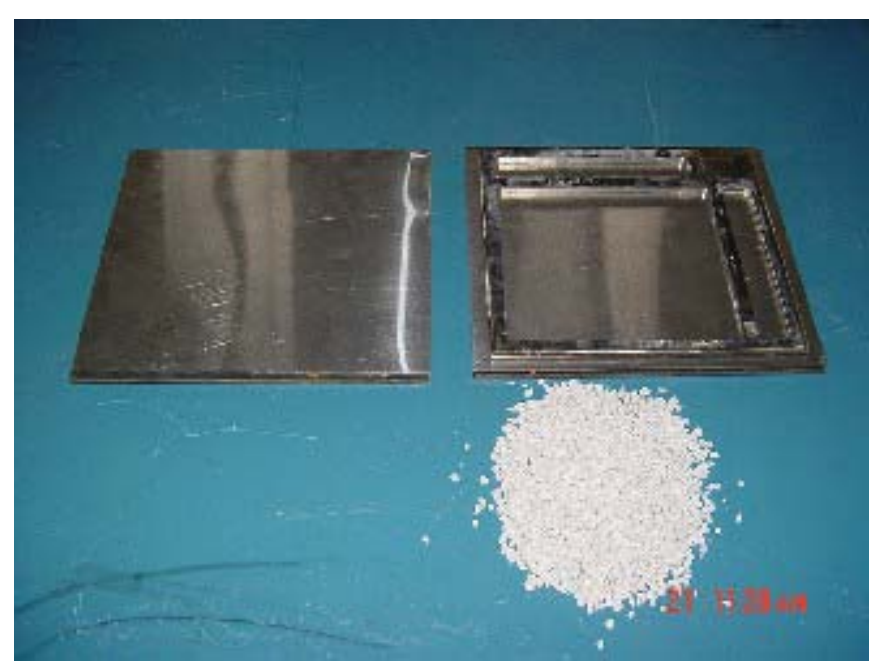

Figure 4.15 Tab Mold, Prepared for Molding

Placing of ABS pellets: For a target density of $67 \mathrm{pcf}$ of compression molded resin, 29 grams (1.02 oz.) of ABS pellets were placed in each of the side slots and 212 grams (7.48 oz.) in the bigger square (Figure 4.16). 


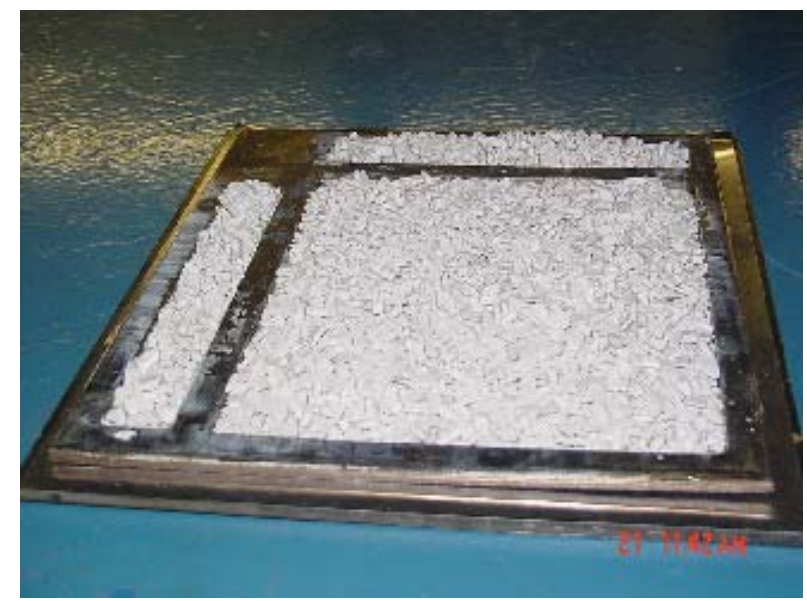

Figure 4.16 Mold for Tab Manufacturing With Recycled Pellets

Molding of Tabs: Mold with pellets and plates were placed on the bottom hot platen of the molding machine, heated to $400^{\circ} \mathrm{F}$. Top plate was then positioned over the pellet pile and bottom platen was pumped until mold made contact with the upper hot platen. At this point pressure began to be applied to the pellets that forced the plates into the mold slots, pressure application was continued until 20 Ton pressure was applied and retained at that pressure for 5 minutes. After 5 minutes of pressure application mold was taken out for cooling for 15 minutes and a fan was used to accelerate cooling. Finally, top and bottom plates were removed and ABS molded tabs were taken out. Figure 4.17 shows the molding process

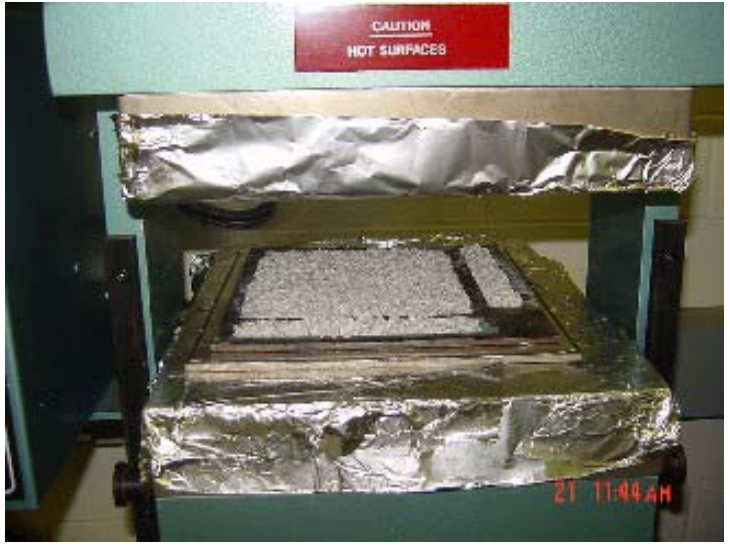

a) Mold in molding machine

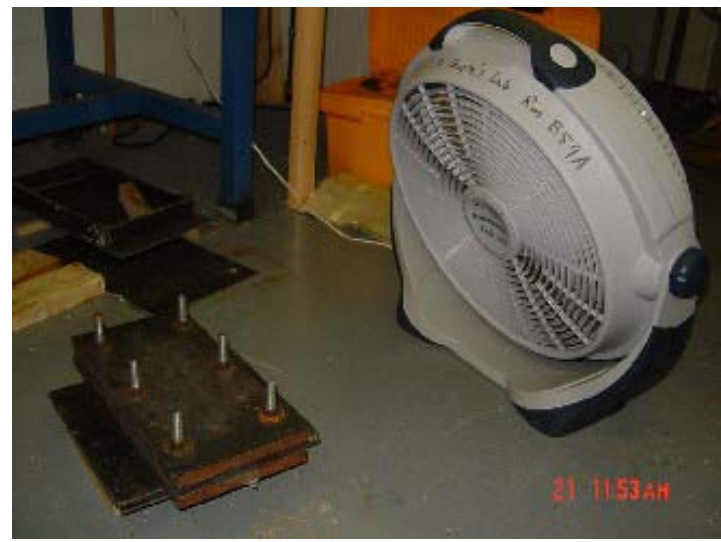

b) Cool Down 


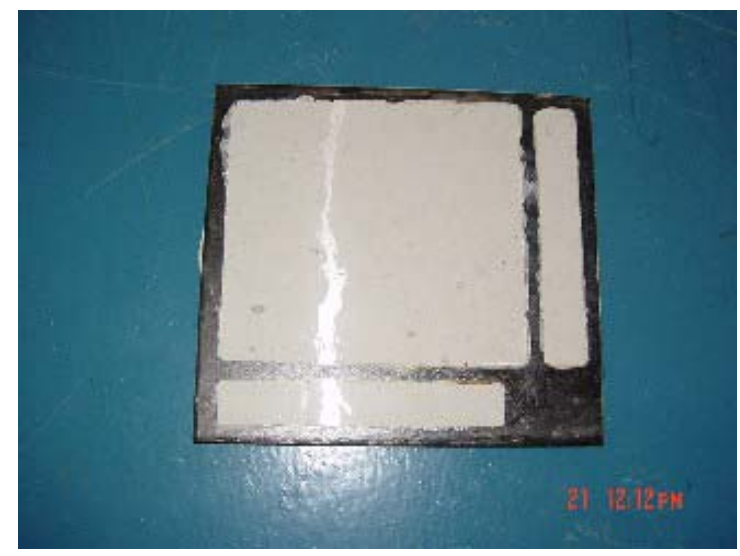

c) Molded tabs before de-molding

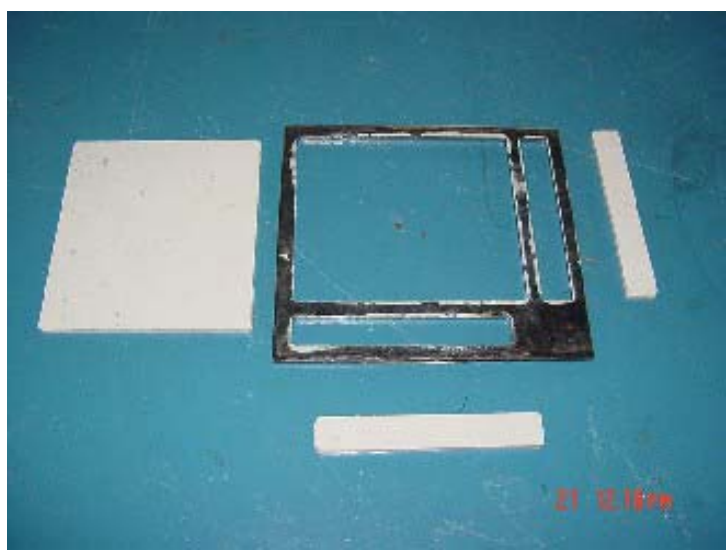

d) Molded tabs taken out of the mold

Figure 4.17 Molding of Tabs

For each module, the manufacturing operation has to be repeated 4 times to get enough ABS tabs to put all around the rubber tire core before wrapping with fabric.

\subsubsection{Molding of Offset Block Module}

Preparation of mold: A square steel frame with inner dimensions of 14" x 8", and a height of 2" was used. Thickness of mold walls was $1 / 2$ ". The four walls were detachable to make it easy to de-mold the product after curing, by just releasing the bolts. Mold was cleaned and coated with oil on the inner walls for easy de-molding of the product. Top and bottom plates were covered with an aluminum flashing to protect plates against extra resin flow and provide a smooth finish to the final product. Mold prepared for module manufacturing is shown in figure 4.18 . 


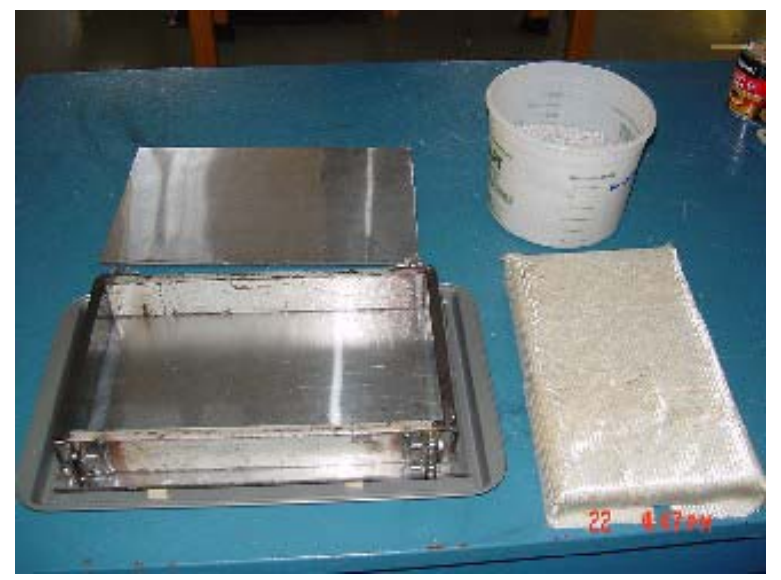

\section{Figure 4.18 Mold Prepared for Module Manufacturing (Wrapped Core Also Shown)}

Preparation of rubber core: Using a reciprocating saw with a blade capable of cutting through high resistance steel, rectangular rubber strips (of size approximately 13" x 7") were cut from discarded rubber tires. Depending upon the tire selected, thickness usually varies between $1 / 2$ " and $1 \frac{1}{2}$ ". During the cutting operation, dimensions can vary $\pm 1 / 2$ " along the edges of the strips. In order to have a top and bottom clear cover of $1 / 2$ " of resin, one or two rubber strips can be used based on the thickness, as shown in Figure 4.19. Volume of the rubber strips with varying thickness was calculated using the average dimensional measurements in order to estimate required amount of $\mathrm{ABS}$ pellets to completely fill the mold.

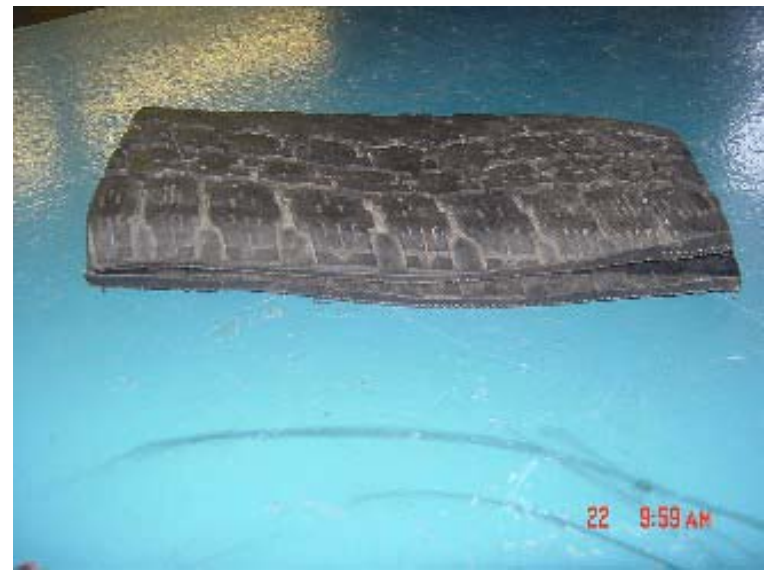

Figure 4.19 Rubber Tire Strips for Module Core 
ABS molded tabs were cut as necessary to completely cover the rubber strips. Bottom tabs were placed first, followed by lateral plates and upper plates. Compatible glue was used for holding the tabs in place while assembling the core as shown in Figure 4.20. It is very important to use as many as required tabs to get a homogeneous melted resin inside the fabric wrap. An approximate volume of the total tabs used was measured in order to estimate the amount of pellets to fill rest of the mold.
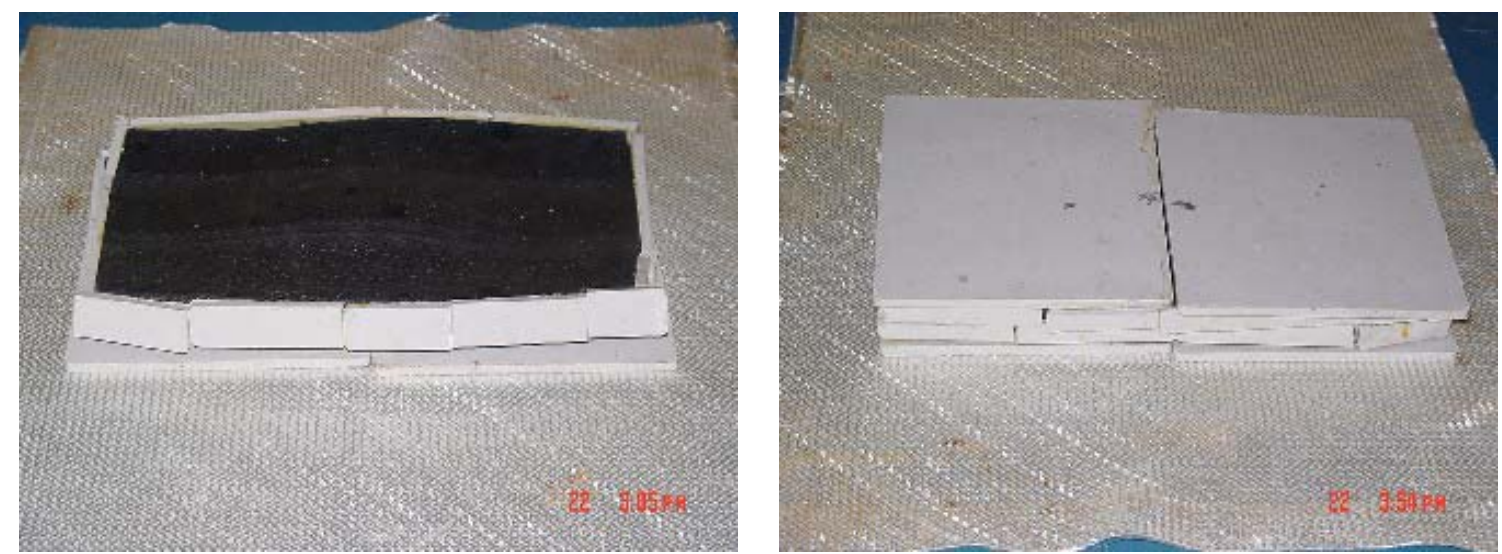

Figure 4.20 ABS Tabs Covering Rubber Core

Glass fabric was then cut as needed and wrapped around the rubber-ABS tabs core. Instant glue was used to hold the fabric in place during this operation (Figure 4.21)

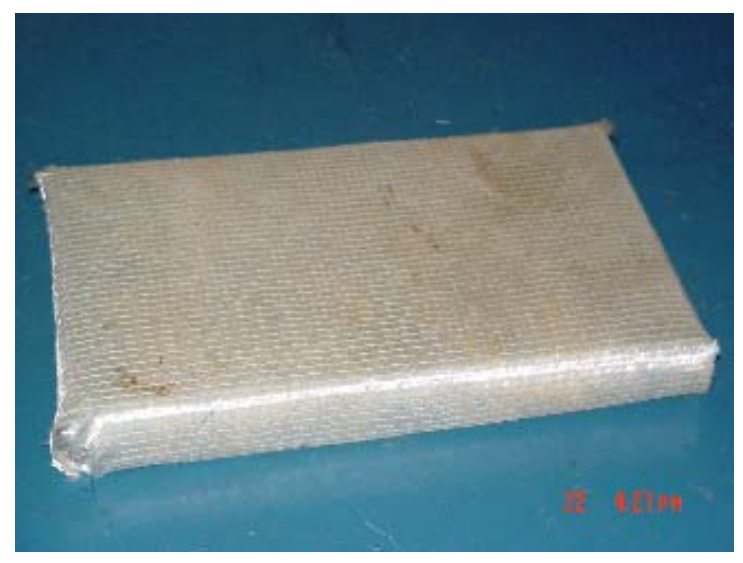

Figure 4.21 Wrapped Rubber-ABS Core

Filling of mold: Wrapped block was placed inside the mold and centered with respect to the walls of the mold. With a target density of $67 \mathrm{pcf}$ for compression molded 
ABS, weight of pellets to be poured in to the mold was calculated by subtracting the volume of the wrapped core from the volume of the mold. Gaps were carefully filled with pellets and remaining pellets were piled on top of the wrapped block as shown in figure 4.22 .

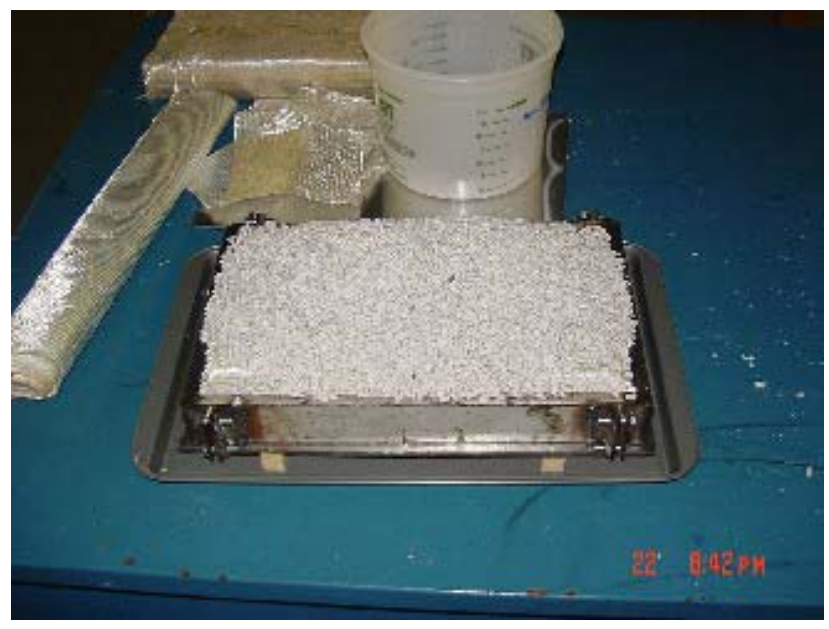

Figure 4.22 Filled Mold

Pre-heating: Pre-heating operation can be described as follows:

- Oven was preheated to $400^{\circ} \mathrm{F}$.

- Using proper protective gloves tray containing mold and bottom plate was carefully transferred to the oven, top plate was separately put inside the oven (Figure 4.23) for pre-heating as well.

- Mold and specimen were left for 25 minutes until melting temperature of $400^{\circ} \mathrm{F}$ was reached. At this temperature pellets mantain their original shape but become soft and sticky and act as a single unit.

- After molding temperature was reached, mold was taken out of oven and placed in molding machine. 


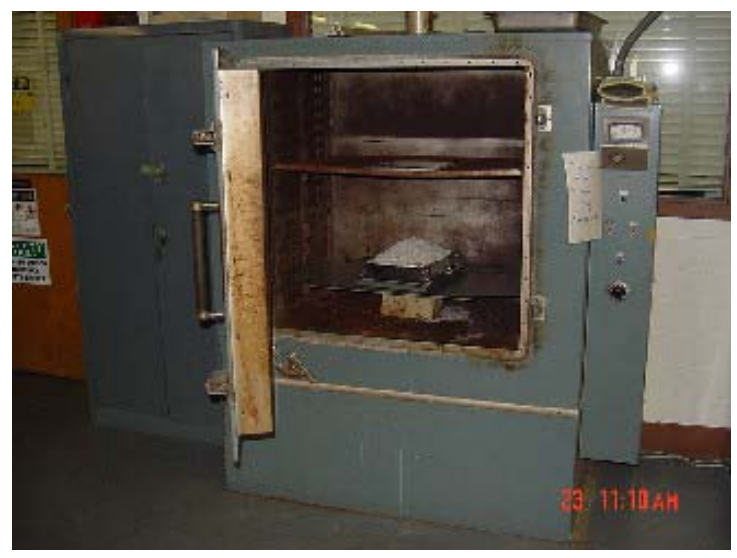

Figure 4.23 Pre-heating Procedure

Compression molding: Top mold plate was put on top of the pellet pile and aligned with respect to the bottom mold plate. Whole of the mold and plate assembly was carefully placed and centered on the bottom hot platen. Bottom platen was pumped up until top mold plate made contact with the upper hot platen. Pumping was continued until 20 Ton pressure was applied and the pressure was held constant for 10 minutes. Following the molding, pressure was released and mold was taken out, placed over wooden supports on the floor, and cooled with the help of a fan for 15 minutes. Finally, top and bottom mold plates were removed, and the mold was disassembled. Offset block module as shown in Figure 4.24.

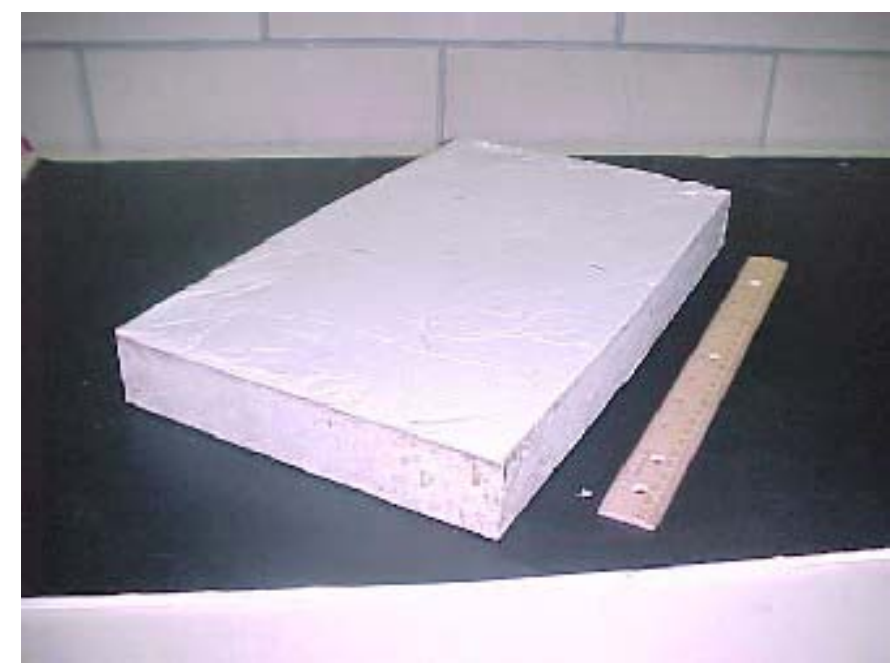

Figure 4.24 Molded Offset Block Module 


\subsection{Testing}

In the following sections, a description of tests carried out is given, including specimen, specimen preparation, test set up and procedure.

\subsubsection{Bending Test}

\subsubsection{Test Specimens}

Five types of FRP specimens were tested under bending as described in Table 4.1. All of the samples were made of recycled resins with the exception of the virgin vinylester box section.

Table 4.1 Types of Bending Specimens for Guardrail Systems

\begin{tabular}{|c|c|c|c|c|}
\hline Section & $\begin{array}{c}\text { Recycled } \\
\text { Resin }\end{array}$ & $\begin{array}{c}\text { Fiber } \\
\text { volume } \\
\text { fraction } \\
\mathbf{( \% )}\end{array}$ & Length & Test- Type \\
\hline Channel & Polypropylene & 43 & $16^{1 / 2}$ & 3-point bending \\
\hline Trapezoidal & Polypropylene & 41 & $16^{1 / 2}$ & 3-point bending \\
\hline Box & ABS & 25 & 20 & 3-point bending \\
\hline Box & Virgin & 41 & 20 & 3-point bending \\
\hline $\begin{array}{c}\text { Fabric wrapped } \\
\text { Box }\end{array}$ & ABS & 22 & 20 & $\begin{array}{c}\text { 3-point bending } \\
\text { 4-point bending }\end{array}$ \\
\hline $\begin{array}{c}\text { Flat specimens } \\
\text { cut from sheets }\end{array}$ & ABS & 25 & $\begin{array}{c}4 \text { (small coupons) } \\
12.75 \text { (big coupons) }\end{array}$ & 3-point bending \\
\hline
\end{tabular}

* Not a recycled resin

For channel and trapezoidal beams a span of 14" was used and for box beams a span of 16" was used as recommended on ASTM D790-92. Figures 4.25, 4.26 and 4.27 show cross section for these specimens. Two types of flat specimens cut from recycled ABS sheets (12.75 in $x 3$ in $x$ 3/8 in) were used as shown in figure 4.28. Dimensions of these specimens were: 
- Plates: 4" long x $3 / 4 "$ " wide x $3 / 8$ " thick

- Half sheet: $12.75^{\prime \prime}$ long x $1.5^{\prime \prime}$ wide x $3 / 8^{\prime \prime}$ thick

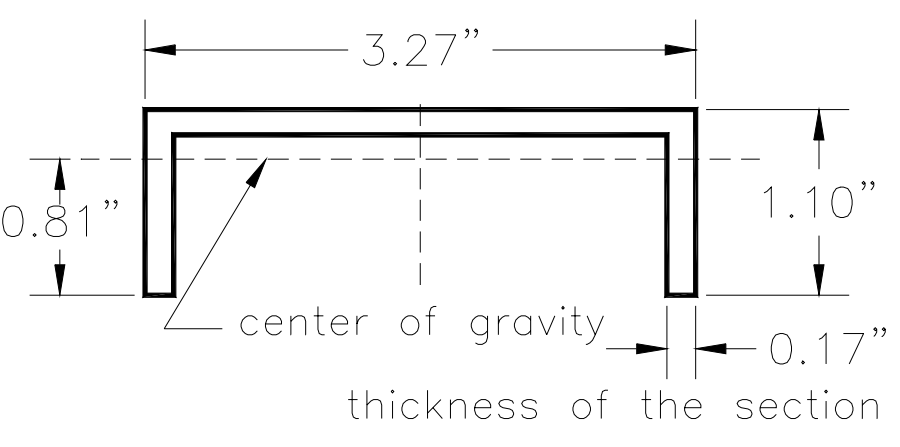

Figure 4.25 Channel Section Dimensions of Propylene Specimen

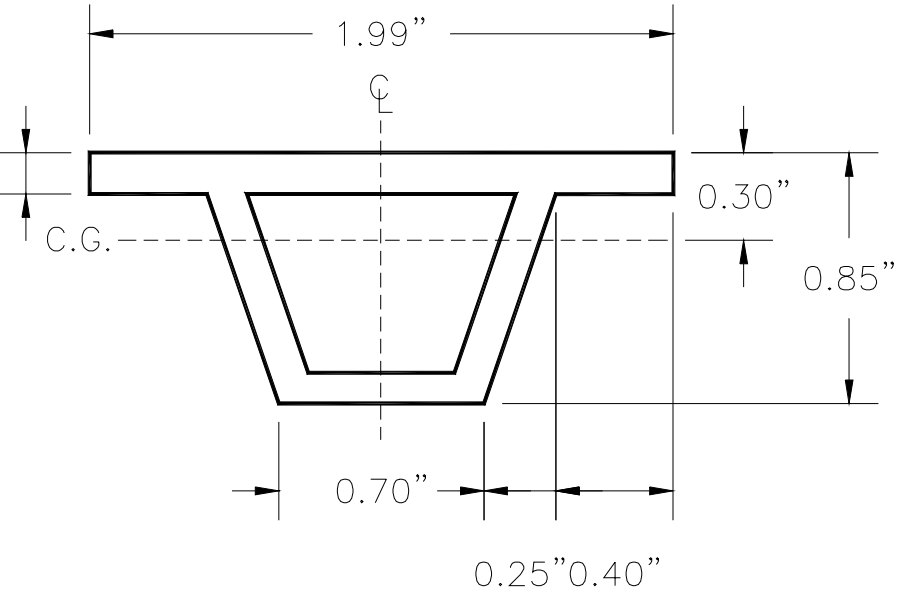

Figure 4.26 Trapezoidal Section Dimensions of Propylene Specimen

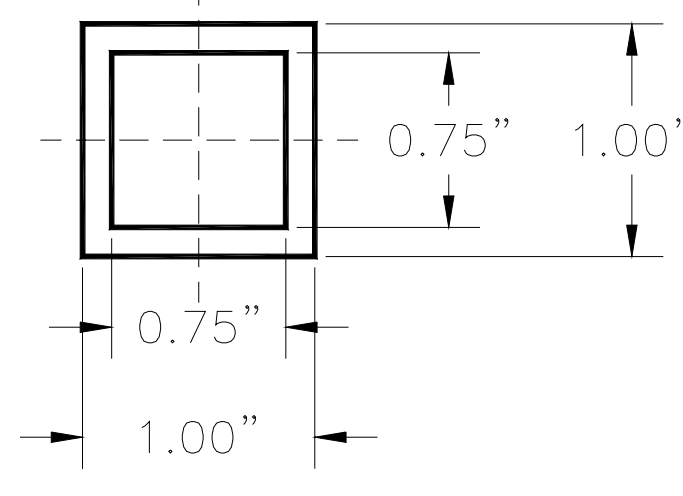

Figure 4.27 Box Section Dimensions of ABS Specimen 


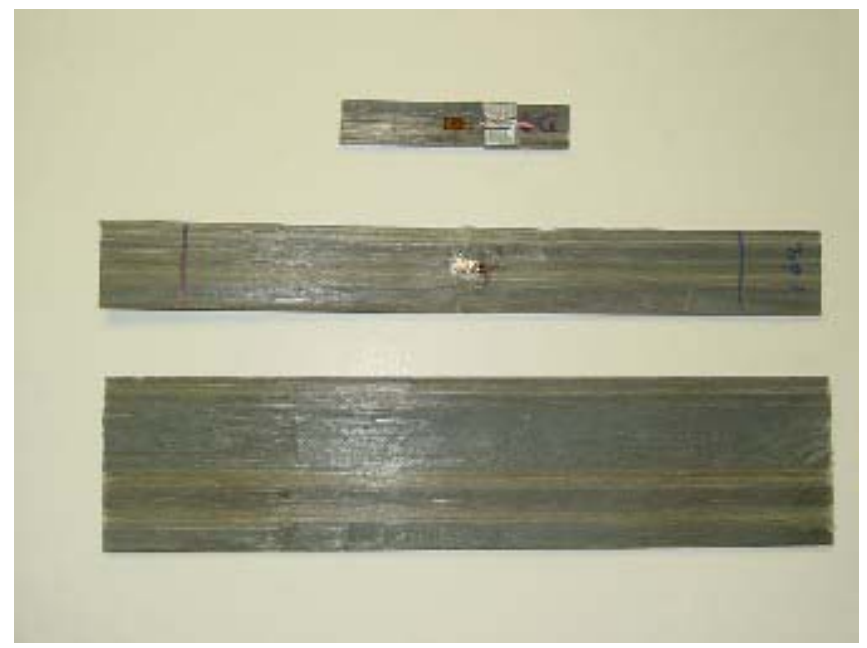

Figure 4.28 Short Plate and Long Plate Bending Specimens (With Strain Gage) Cut from Recycled ABS Sheets (Also Shown)

\subsubsection{Specimen Preparation}

For each of the shapes tested, strain gages were installed as follows:

- 5 gages for channel section (Fig. 4.29)

- 4 gages for trapezoidal section (Fig. 4.30)

- 2 gages for box section (Fig 4.31)

- One gage for ABS small plates on the compression side at midspan (Figure 4.28)

- 2 gages for ABS long plates at midspan one on the compression and one on the tension side. (Figure 4.28 shows only one side) 


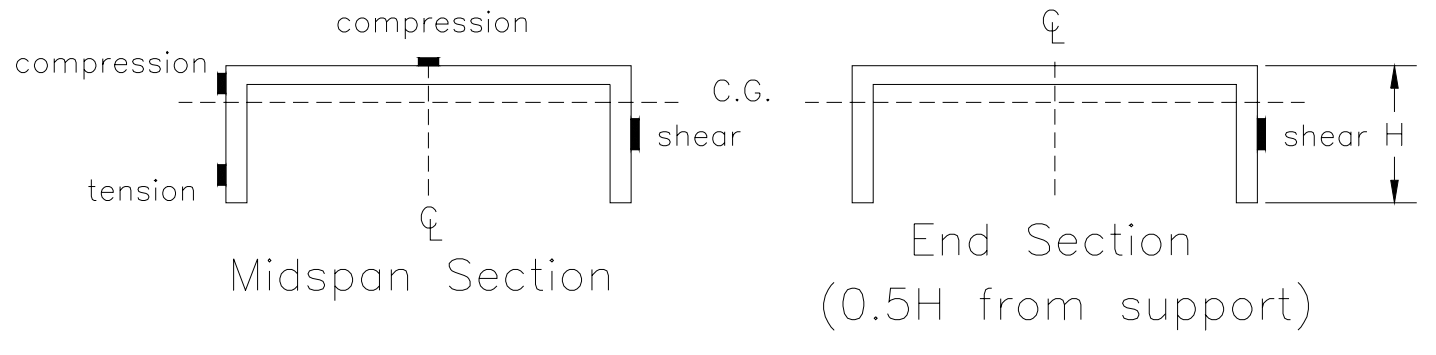

Figure 4.29 Strain Gage Locations for Bending Test on Channel Section

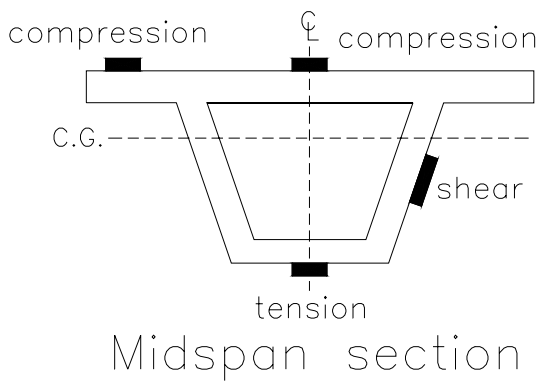

Figure 4.30 Strain Gage Locations for Bending Test on Trapezoidal Section

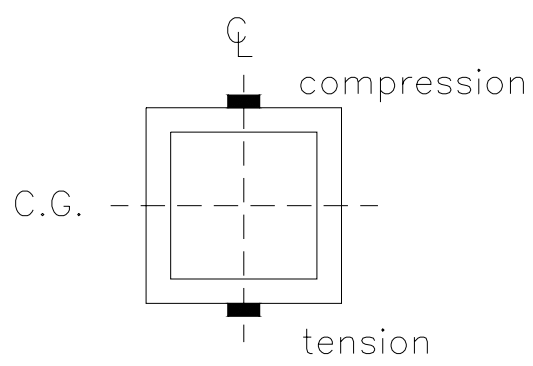

Figure 4.31 Strain Gage Locations for Bending Test on Box Sections (Midspan)

Surface preparation for strain gage bonding consisted of the following steps:

1) Sanding with a 320 grit sandpaper

2) Cleaning with an acid surface cleaner (degreaser)

3) Cleaning with water-based conditioner and neutralizer solutions to remove oil residues and help in the adhesion of the strain gage

4) Bonding the gage to the required specimen surface using M-Bond 200 adhesive 
5) Allowing the adhesive cure overnight with proper clamping pressure to ensure adequate bond

Box sections were tested with and without glass fabric wrapping. Box sections required an additional surface preparation for glass fabric wrap installation as described below:

1) To ensure good adhesion, all four surfaces were lightly ground to remove the glossy finishing given during manufacturing process

2) Mbrace Primer supplied by Master Builders Technologies was applied on all of the surfaces

3) Resin was cured for 48 hours

4) A thin layer of Mbrace Saturant resin was applied

5) Fabric was coated with resin and wrapped around the specimens

6) A final coat of resin was applied
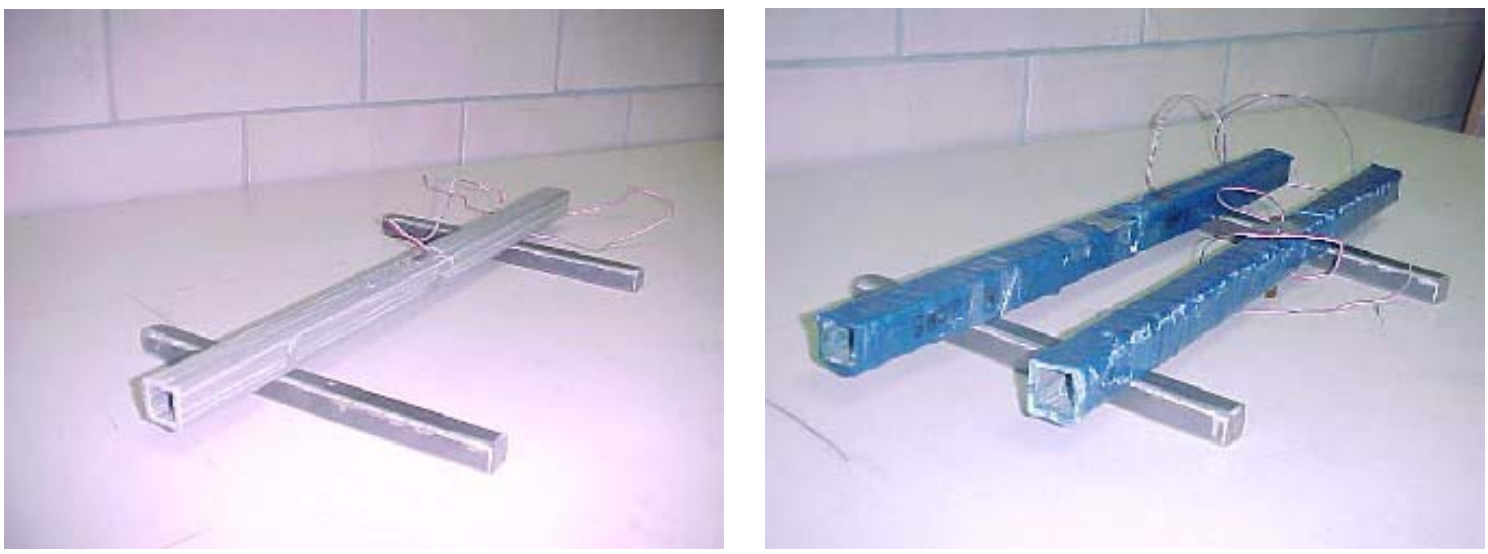

Figure 4.32 Non Wrapped and Wrapped Box Specimens for Bending Test

A wooden stiffener was placed inside the channel section (Figure 4.33) at both ends of the specimen, to prevent shear/punching failures at support points. 


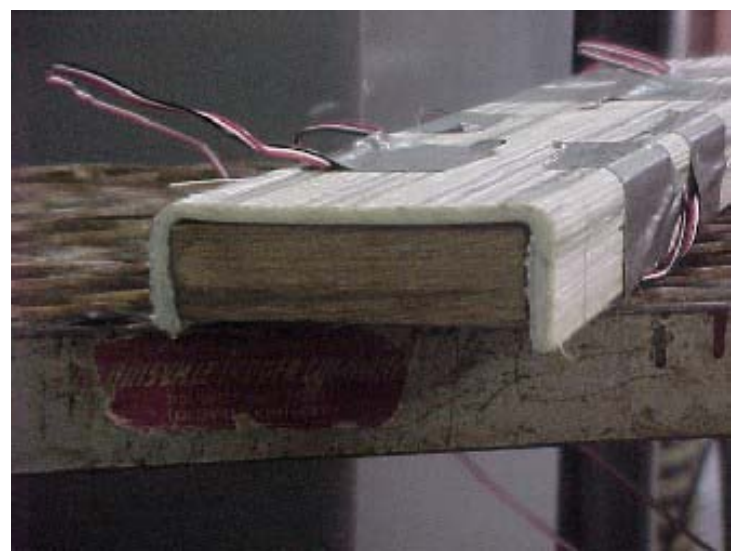

Figure 4.33 Wooden Stiffeners at Ends of Channel Section

\subsubsection{Test Set-up and Procedure}

Universal testing machine (UTM) shown in figure 4.34, was used to conduct the bending tests in channel, trapezoidal and box sections. ABS strips were tested as described on section 3.5.2.3. UTM was set to a low loading range of 0 to $10 \mathrm{kips}$. Test was carried out at a constant rate of $\approx 0.25 \mathrm{in} / \mathrm{min}$ until the test specimen failed. Deflections were manually recorded from a dial gage placed at midspan of the specimens, whereas load and strain readings were automatically recorded with a data acquisition system. Bending test set-up and specimens are shown in Figures 4.35 to 4.39.

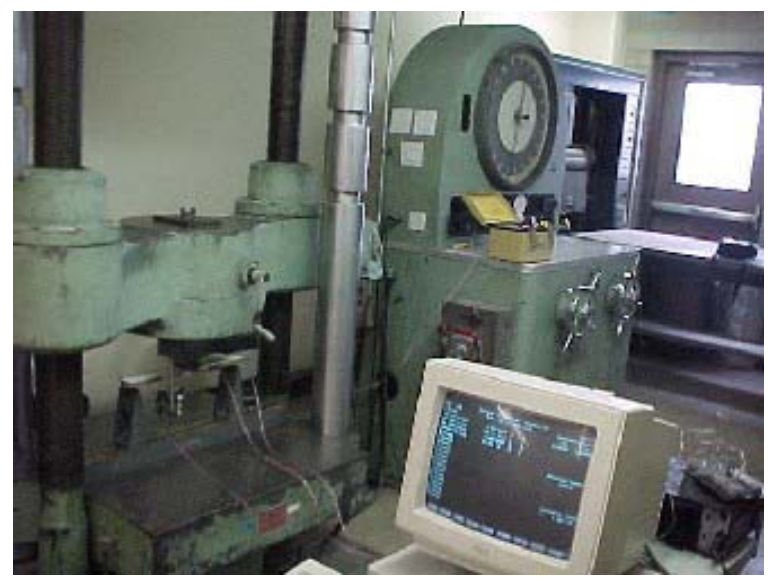

Figure 4.34 Bending Test Set-up: Universal Testing Machine and Data Acquisition System 

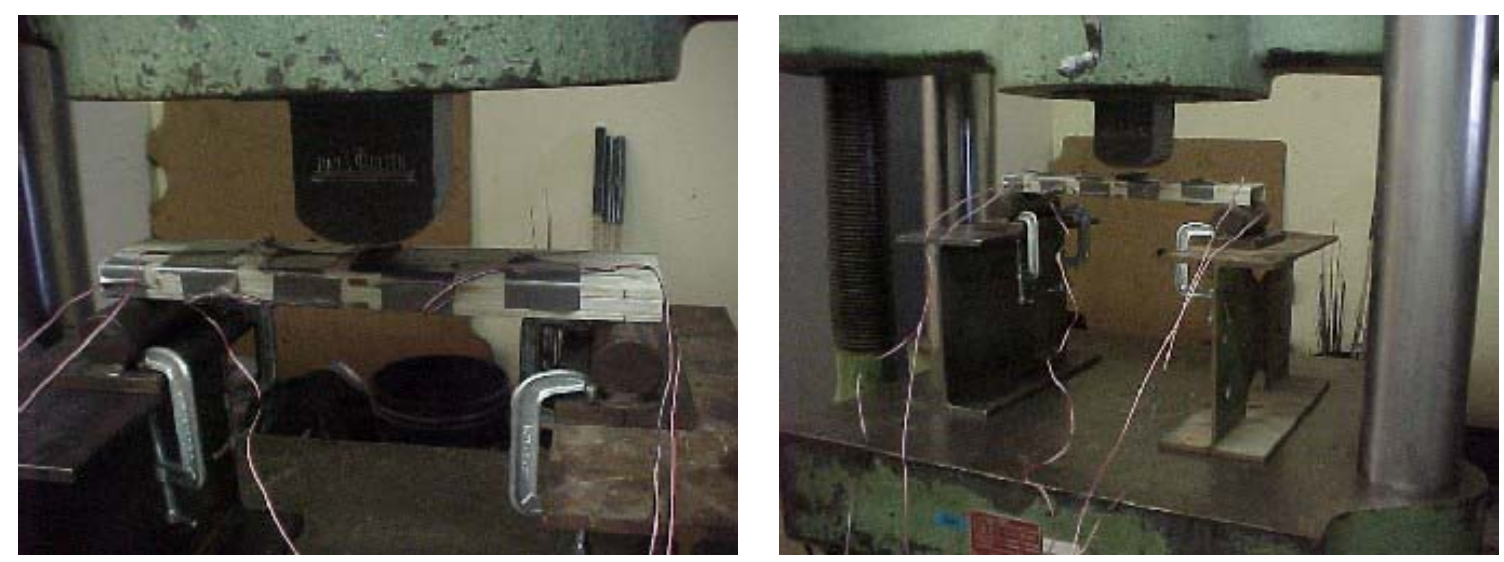

Figure 4.35 Channel Section Set-up for Bending Test
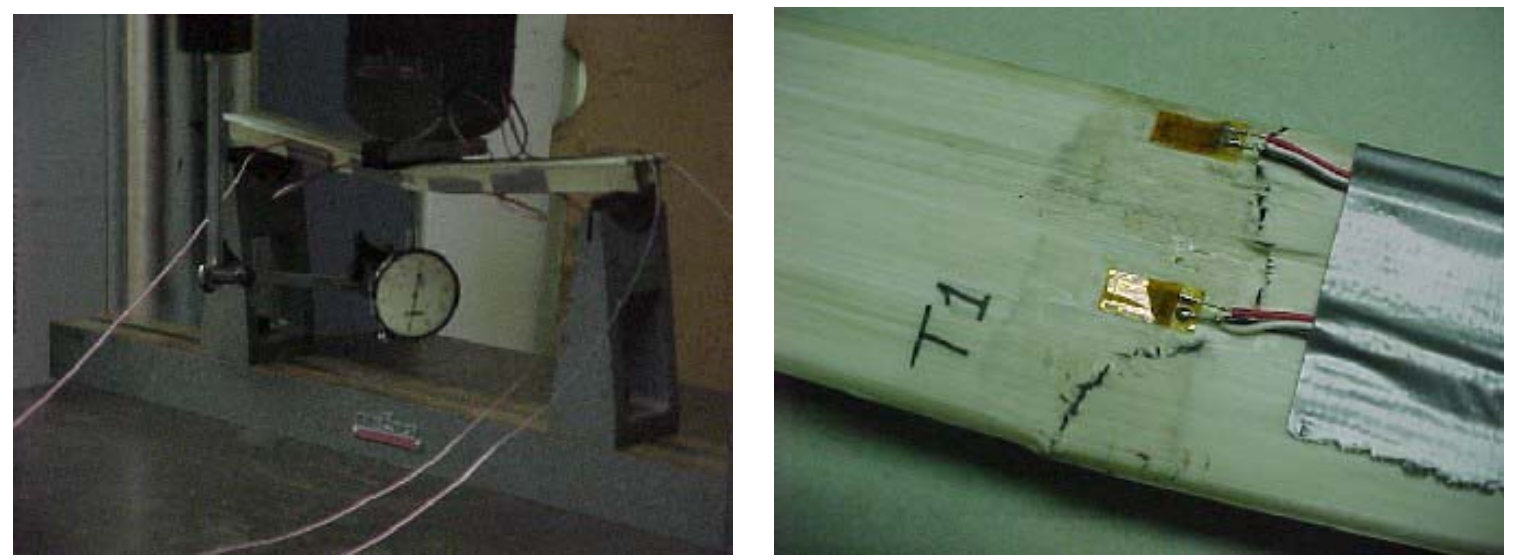

Figure 4.36 Trapezoidal Section Bending Test Set-up and Failure Mode
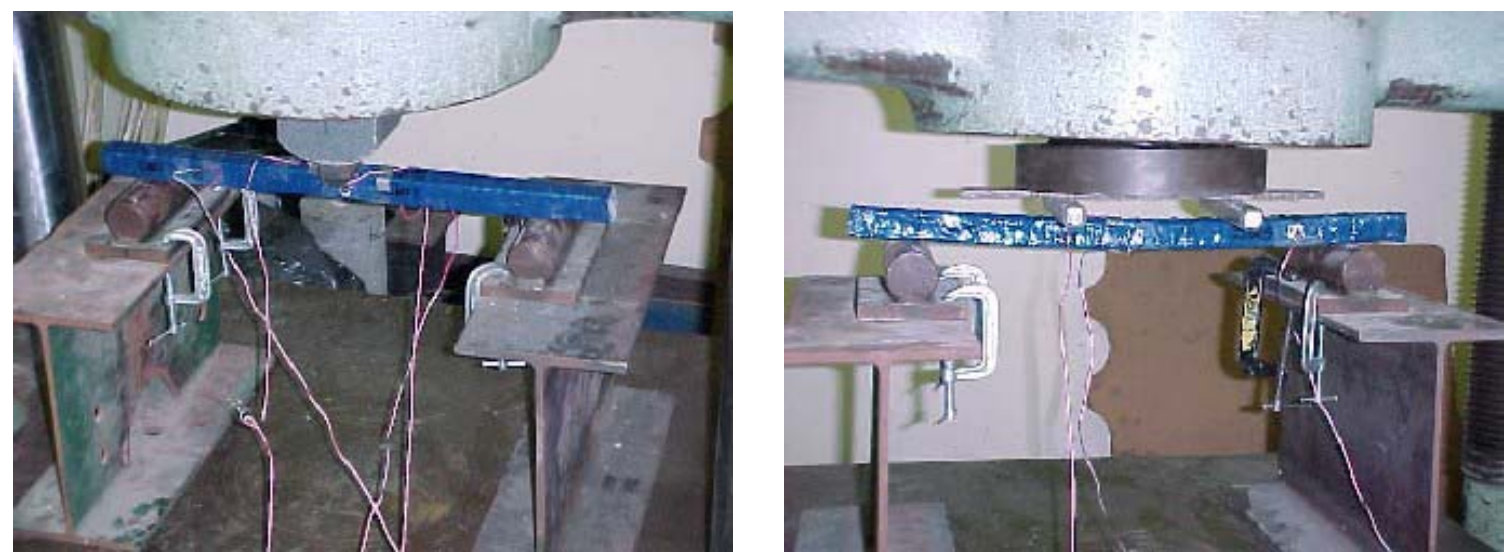

Figure 4.37 Wrapped Box Section Three and Four Point Bending Test Set-up 


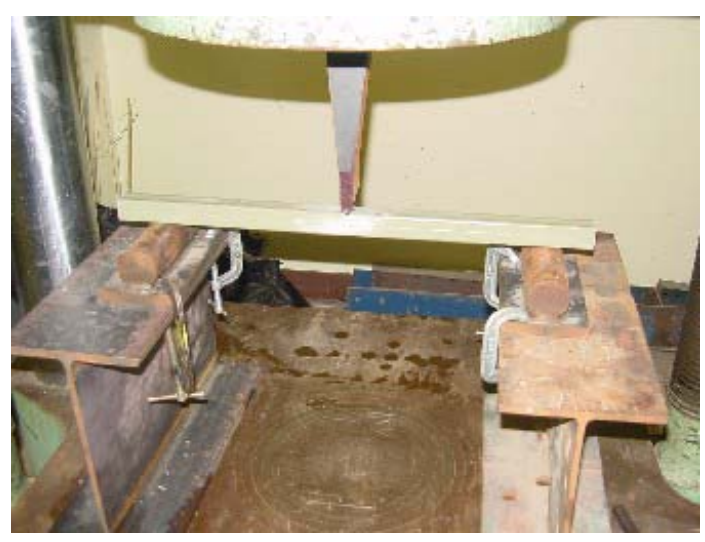

Figure 4.38 Virgin Vinylester Box Section Three Point Bending Test Set-up

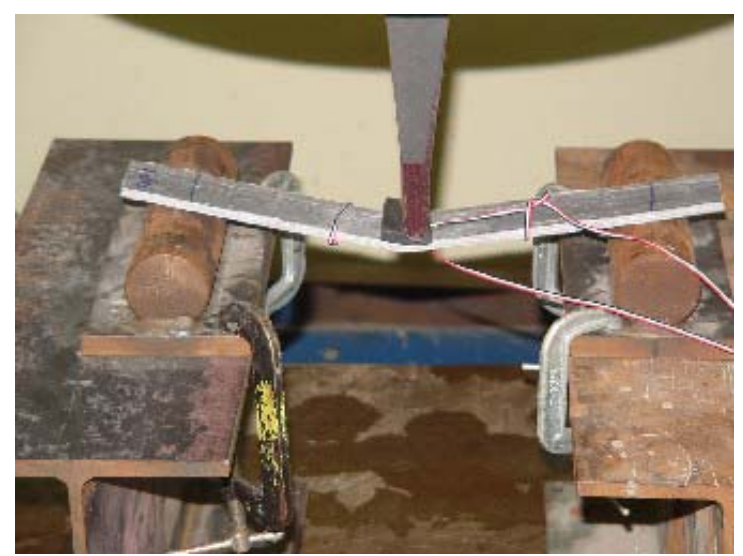

Figure 4.39 Recycled ABS Half Sheet Specimen Three Point Bending Test Set-up

\subsubsection{Tension Test}

\subsubsection{Test Specimen}

Strips cut from trapezoidal sections, ABS sheets, ABS belt-type specimens (flexible) and ABS box sections were tested under tension. For polypropylene samples from trapezoidal sections (Fig. 4.40), typical strip dimensions were 7" x 1/2" with variable thickness from 0.08 " to $0.135 "$ depending upon its location on the section from where it was extracted. For strips from ABS sheets (Fig 4.41), dimensions were 7 " x 1/2" and thickness of 3/8". For strips from ABS belt-type specimens (flexible) (Fig 4.41 and 4.42), dimensions were 7" x 1/2" and thickness was around 0.01 ". For ABS box specimen, length 
was $42 "$ and cross sectional dimensions were as shown in Fig. 4.3. Dimensions were selected based on ASTM D638-94b recommendation.

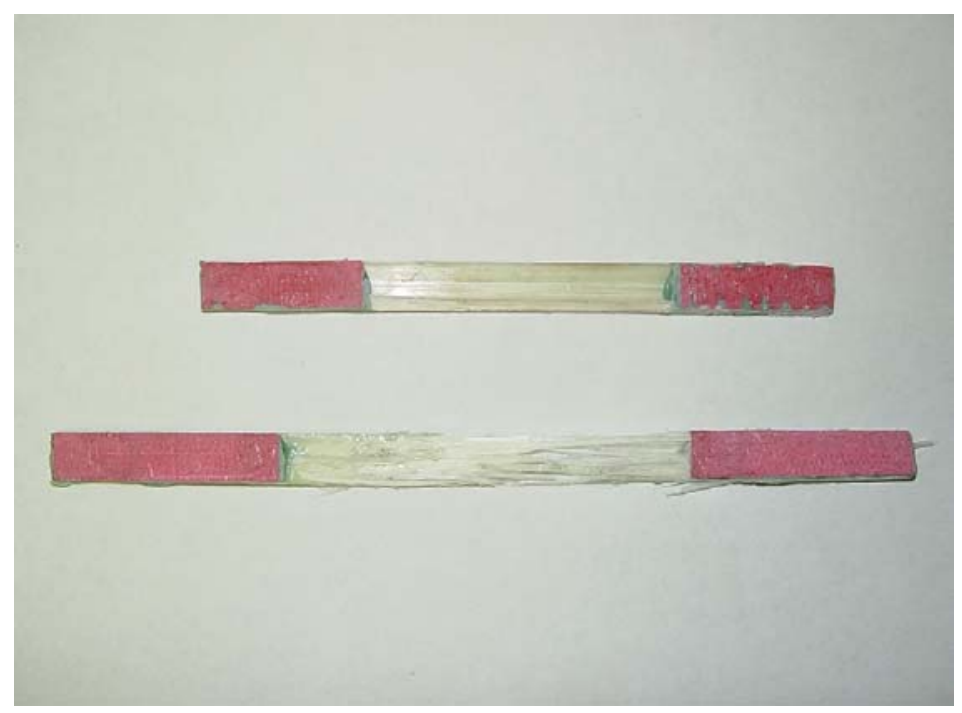

Figure 4.40 Polypropylene Strip Specimen from Trapezoidal Sections Used for Tension Tests (Before and After Testing)

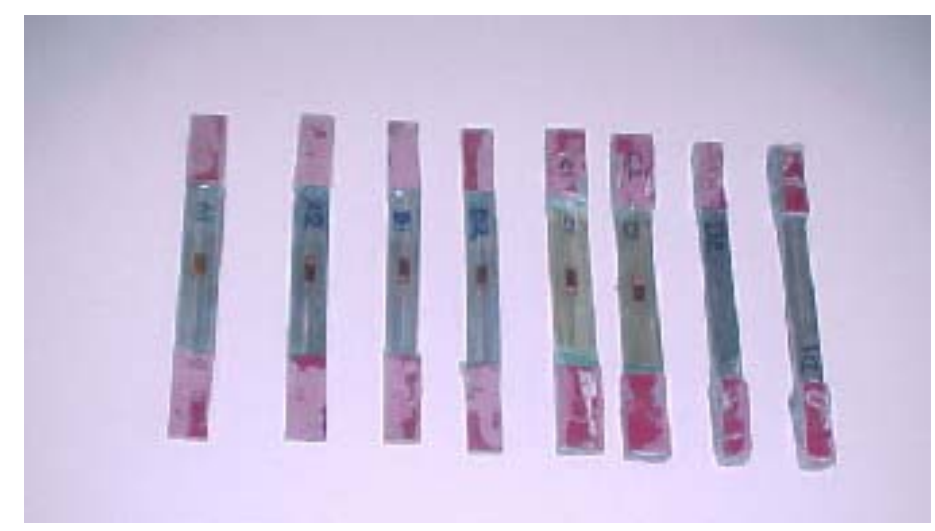

Figure 4.41 ABS Strip Tension Specimen from Belt Type (Flexible) Product and from Sheets (Last Two on the Right) 


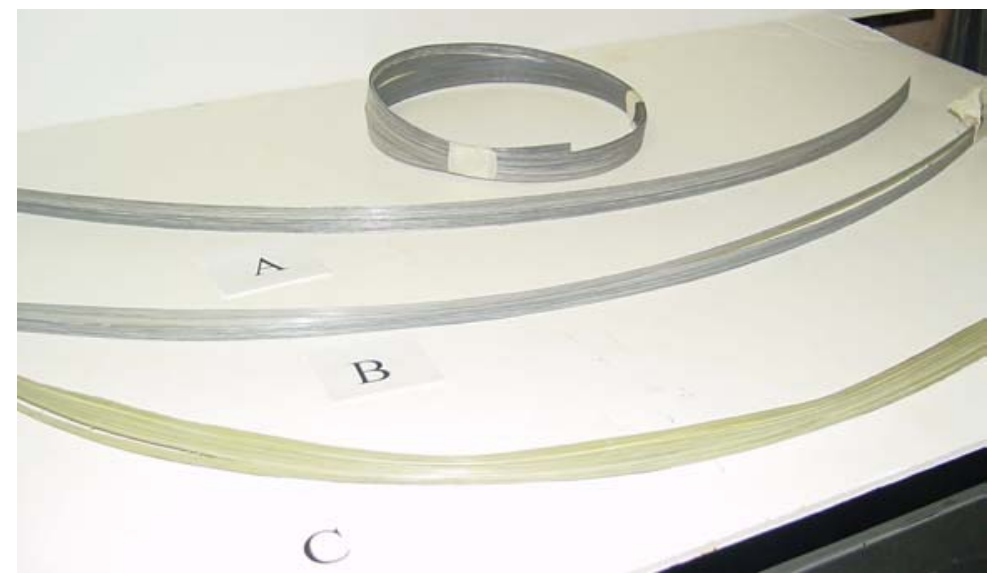

Figure 4.42 ABS Belt Type (Flexible) Product

\subsubsection{Specimen Preparation}

To prevent slipping of the specimen from the grips of testing machine and for effective force transfer during tension testing, plastic grips were attached to the specimens. Each of the grips was 2" long for strips and 12" long for box specimens. Details of grip preparation are as follows:

1) Grip length was lightly ground and grooves were cut on bonding area of both specimens and plastic tabs using a mechanical grinder or a file.

2) Tabs (grips) were bonded to specimens using Ashlands Plyogrip adhesive. The glue was applied and allowed to cure for at least 48 hours under pressure application by means of weights (for strips) or clamps (for box specimens).

In order to measure the tensile strains on each specimen, a series 2620 dynamic extensometer with a gage length of 1.00 inch was attached using rubber bands at the midsection as shown in Figure 3.3. Due to the flexibility of belt specimens, it was not possible to attach extensometer. Instead, strain gages were used to measure strains on these specimens. For box specimens, two strain gages were attached at midlength (Fig. 
4.44), rectangular stiffeners were inserted at the grip length of the box section to prevent crushing of the hollow section.

\subsubsection{Test Set-up and Procedure}

Computer controlled Instron 8500 two-column load frame-testing machine was used to test the strip type specimens. Strain readings were taken with a strain indicator unit. "Wavemaker " software was utilized to carry out the tests. Specimens were loaded to failure or until deflections greater than one third of the span were attained at a constant rate of $0.25 \mathrm{in} / \mathrm{min}$. Test set up for tensile strip specimens is shown in Figure 4.41

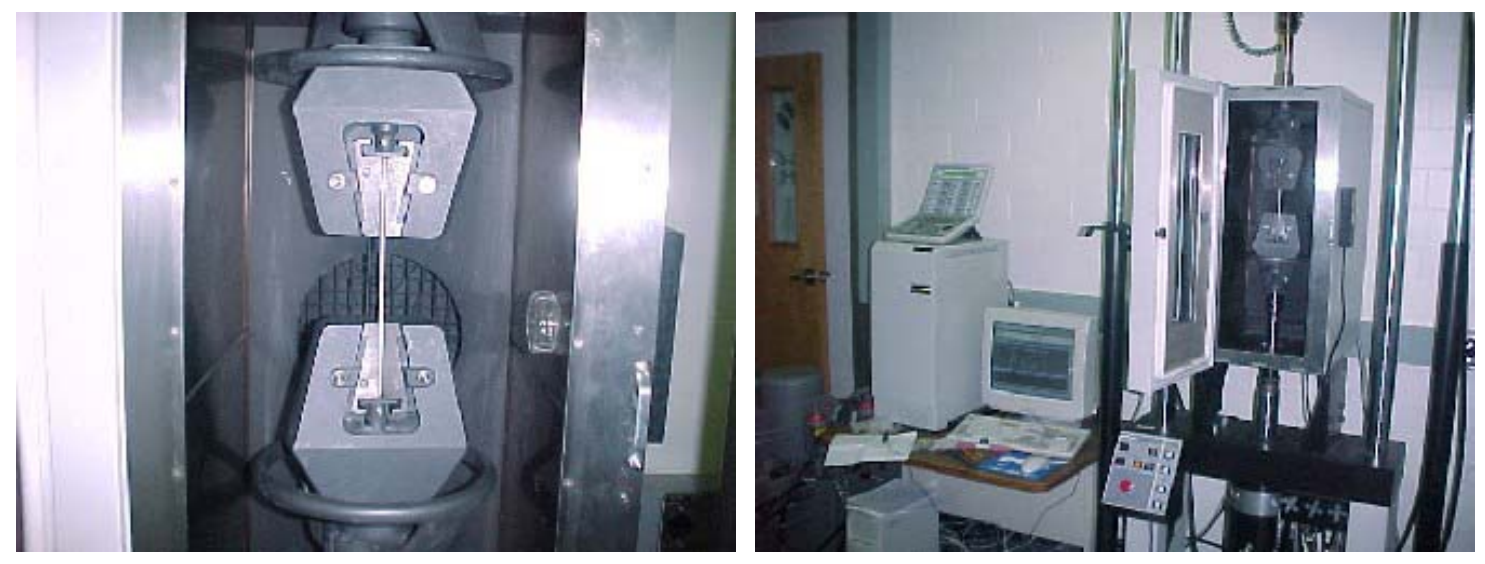

Figure 4.43 Strip Type Specimens Tension Test Set-up

Baldwin testing machine was used to test the box section (Figure 4.44). It was set to medium range loading mode of 0 to 50 kips. Test was carried to failure of the specimen at a constant rate of $\approx 0.25 \mathrm{in} / \mathrm{min}$, load and strain readings were recorded using a data acquisition system. 

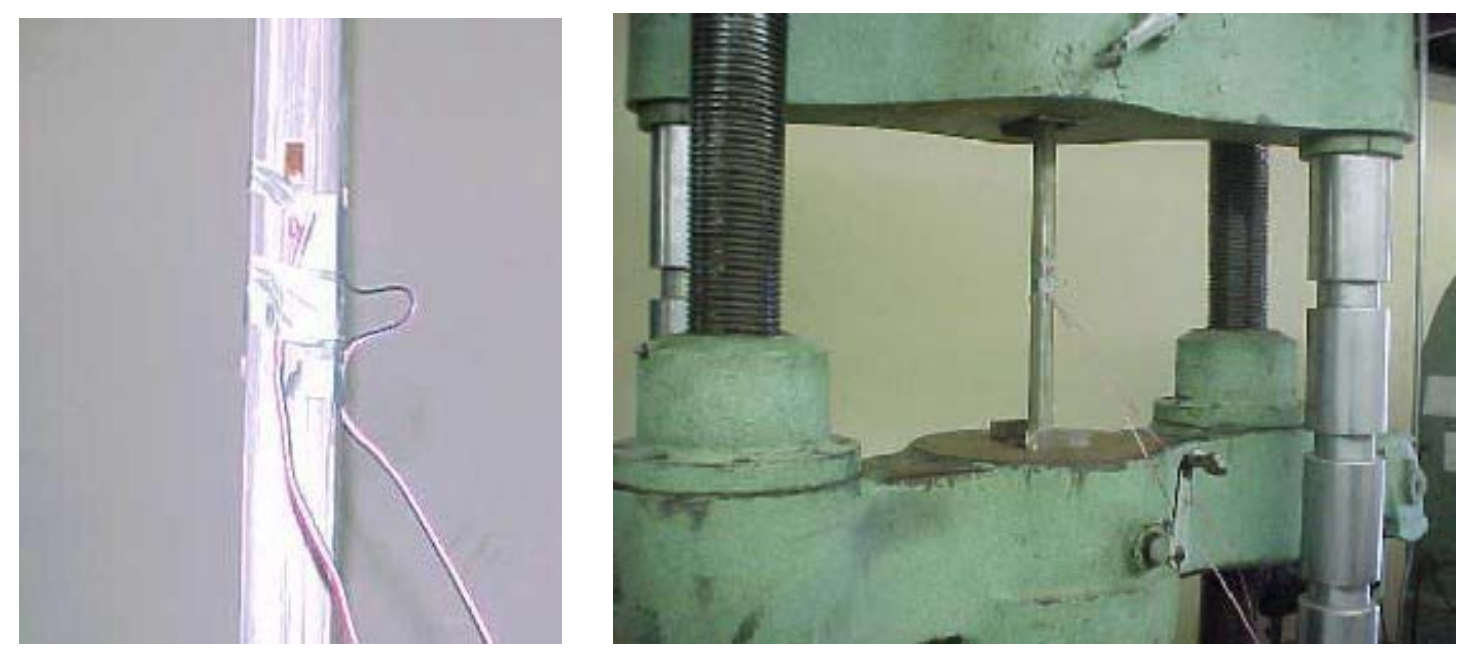

Figure 4.44 Box Specimen Tension Test Set-up

\subsubsection{Compression Test}

\subsubsection{Test Specimen}

Rectangular specimens cut from ABS sheets with cross section of 0.5 " $x$ 3/8" and height of 0.5" were used as per ASTM D695-91.

\subsubsection{Specimen Preparation}

No specimen preparation was necessary for these specimens.

\subsubsection{Test Set-up and Procedure}

Same Instron 8500 two-column load frame-testing machine was used. The set up is shown in Figures 3.7. Specimens were loaded to failure at a constant rate of 0.05 in/min.

\subsubsection{Compression Test of Rubber-Wood Offset Block Model}

\subsubsection{Test Specimen}

As discussed in section 4.3.2, this model consisted of rubber tires and wood. Dimensions of wooden blocks were 91/2" x 6" with a thickness of 2 ", slots were 1" deep. Dimensions of rubber strips were approximately $8 \frac{1}{2} "$ x 7 " with an approx. thickness of $3 / 4$ ". Overall dimensions of the block were $91 / 2 "$ x $6 "$ x $83 / 4 "$ 


\subsubsection{Specimen Preparation}

No specimen preparation was necessary for this test specimen.

\subsubsection{Test Set-up and Procedure}

Baldwin machine (UTM) was used to test the box section. Tests were carried out at an approximate deformation rate of $0.25 \mathrm{in} / \mathrm{min}$. Load readings were manually recorded. The model was loaded until buckling of rubber strips and beyond. Test set-up is shown in Figure 4.45 .

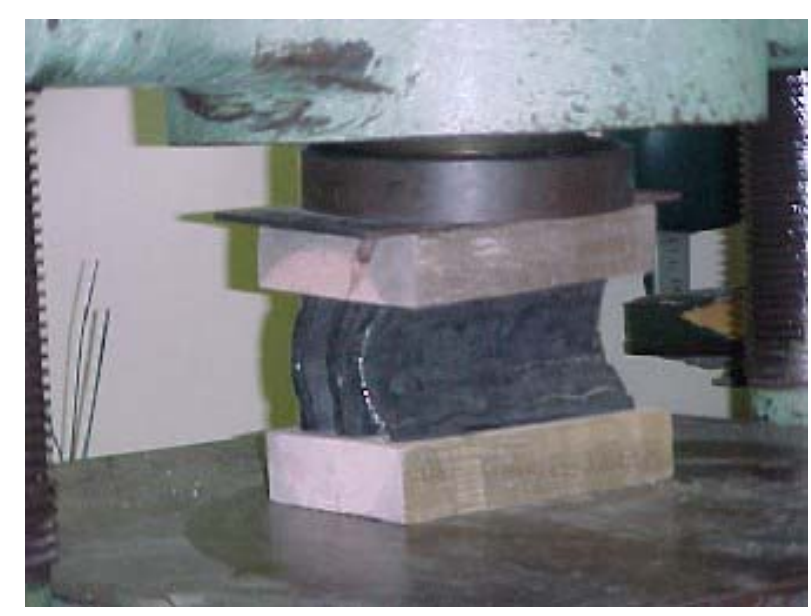

\section{Figure 4.45 Compression Test on Rubber-Wood Offset Block Model}

\subsubsection{Tests on Wood from Rubber -Wood Block Model}

In order to evaluate the strength of wooden blocks used for the rubber-wood model, compression tests parallel to grain and perpendicular to grain (as per ASTM D143-94 procedures) and impact tests were performed on wooden specimens cut from actual prototype rubber -wood offset block.

\subsubsection{Test Specimen}

Compression parallel to grain: Blocks with a cross section of $2 "$ x 2" and a length of 8", placed on the 2" x 2" section were tested as per ASTM D-143-94 
Compression perpendicular to grain: Blocks with a cross section of 2" x 2" and a length of 6", placed on a 2" x 6" face were tested as per ASTM D-143-94

Impact test: Specimens with dimensions described on section 3.5.4.1 and Figure 3.9 were manufactured as shown in Figure 4.46 and tested using the BLI machine described on section 3.5.4.3. Objective of tests is to compare results to those obtained for recycled thermoplastic resins (ABS and PC).

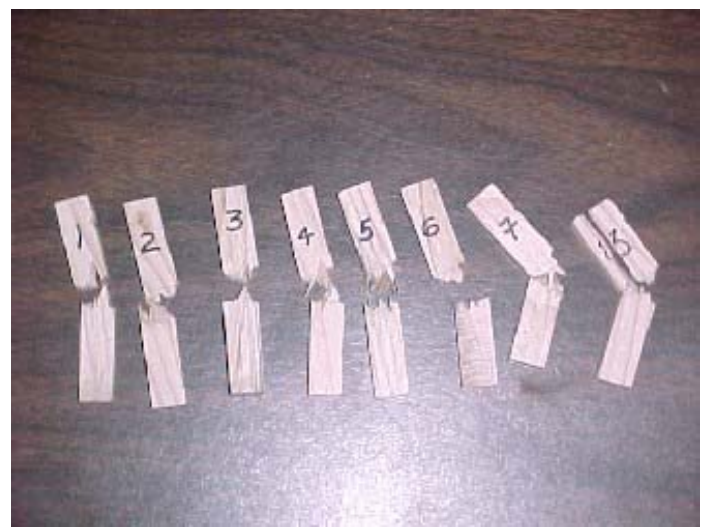

Figure 4.46 Tested Wooden Impact Specimens

\subsubsection{Specimen Preparation}

Strain gage was attached as described on section 4.4.1.2 on each of the compression specimens to measure strains in the direction of the compressive force that was applied on the specimens. Preparation was made following the procedures described on section 4.4.1.2.

Impact specimens were provided with V-notches as per ASTM D25-93a (Figure 3.9).

\subsubsection{Test Set-up and Procedure}

Compression parallel to grain: Initially, Instron 8500 two-column load frametesting machine was used to conduct the compression as shown in Figure 4.47. The test 
was computer controlled with exception of strain readings. The strain readings were recorded by using a strain indicator unit. Specimens were loaded up to $16 \mathrm{kips}$, within the Instron's maximum loading capacity of 21 kips. Tests were continued in Baldwin machine until failure, at a rate of approximately $0.10 \mathrm{in} / \mathrm{min}$.

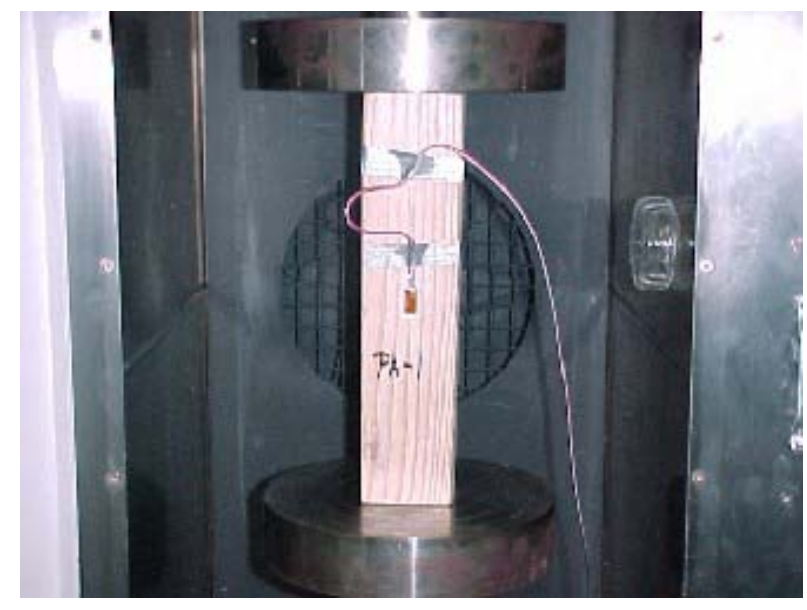

Figure 4.47 Compression Parallel to Grain Test Set-up

Compression perpendicular to grain: Instron 8500 two-column load frametesting machine was used to conduct the compression test as shown in Figure 4.48. The test was computer controlled with the exception of strain readings. The strain readings were recorded using a strain indicator unit. Tests were loaded until failure, at a rate of approximately $0.10 \mathrm{in} / \mathrm{min}$.

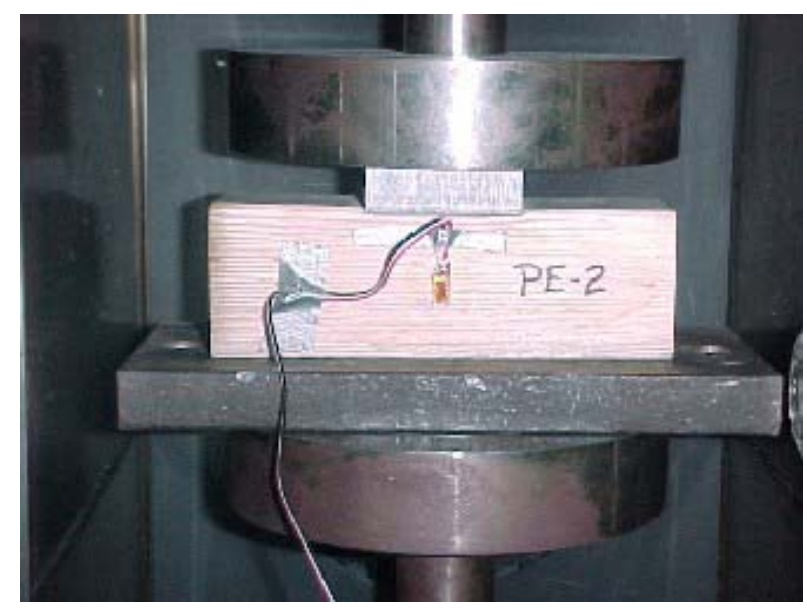

Figure 4.48 Compression Perpendicular to Grain Test Set-up 
Impact test: test set-up and procedure was the same described in section

\subsubsection{3}

\subsubsection{Bond Strength Test for Rubber- ABS Interface}

While manufacturing the offset block for highway guardrail systems, we developed a compression molding process using ABS and rubber. In order to evaluate the bonding strength between rubber and molded ABS, pull out tests were performed. Additional details are given below.

\subsubsection{Test Specimen}

An aluminum apparatus shown in Figure 4.49a was designed and constructed at CFC-WVU to facilitate molding of recycled thermoplastic pellets and samples required for testing. Specimens consisted of ABS molded strip 7" x 11/2" and 1/4" thick with a rubber block from discarded tires $2 \frac{1}{2} "$ × $1 \frac{1}{2}$ " and $1 / 2$ " thick. The mold consisted of a hollow space where the rubber block was placed during molding phase (Fig. 4.49b). A primer was coated on the rubber at the contact surface to improve bonding between ABS and rubber. Inner layer of pellets was first positioned in the hollow space (Fig. 4.49c) and a glass fabric was positioned over this layer (Fig. 4.49d). A final layer of pellets was piled over the fabric (Fig 4.49e). Top plate was placed over the pile of pellets and the specimen was compression molded under 20 Tons for 5 minutes, following the procedure described on section 4.3.1. The specimens are shown in Figure 4.49f and 4.50. 


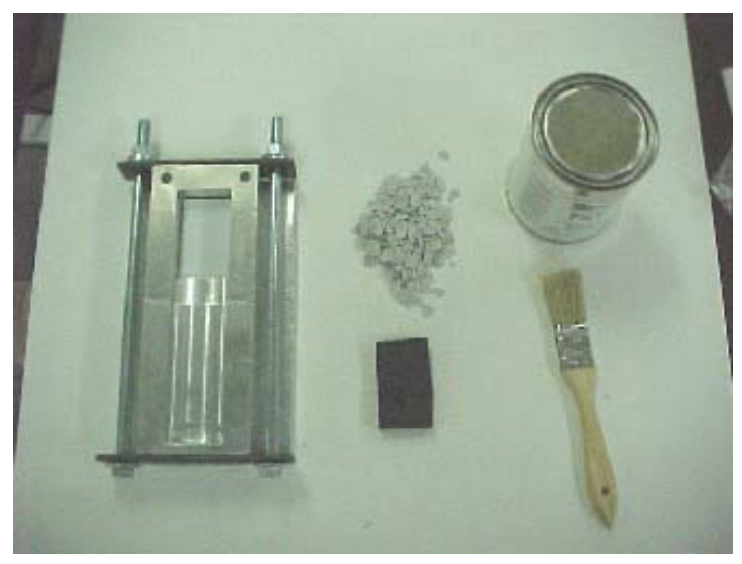

a) Mold and Materials

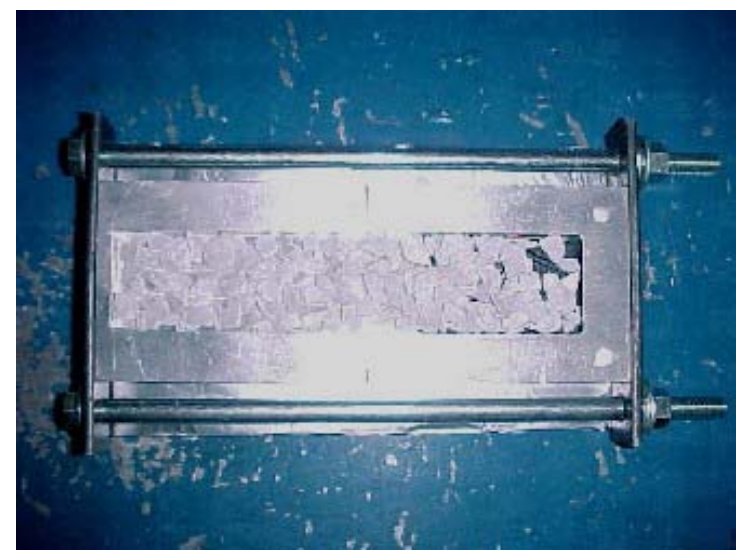

c) Pellet Inner Layer

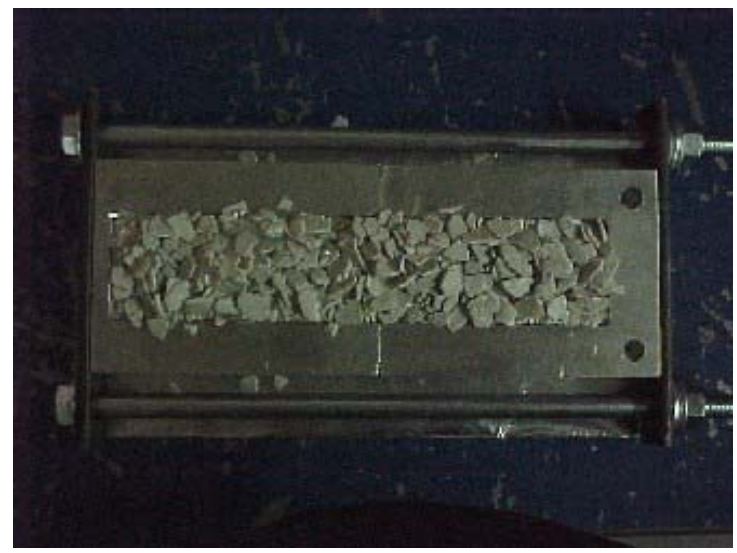

e) Pellet Outer Pile

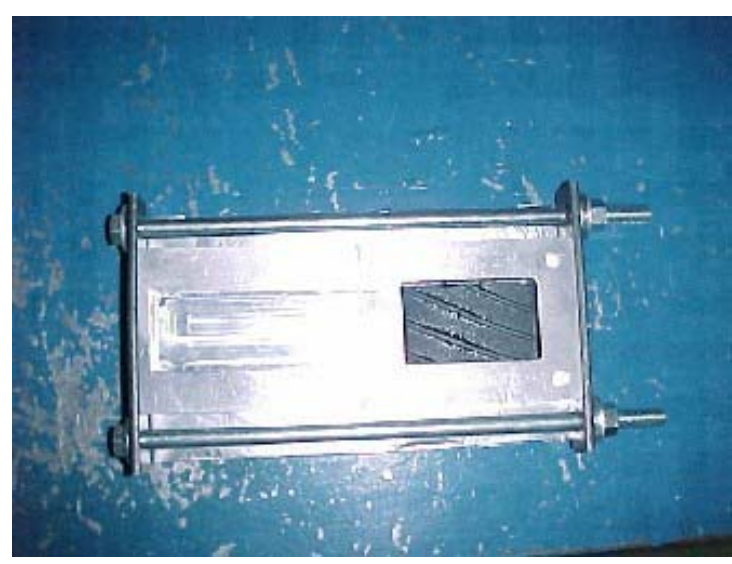

b) Positioning of Rubber Block Coated With Primer

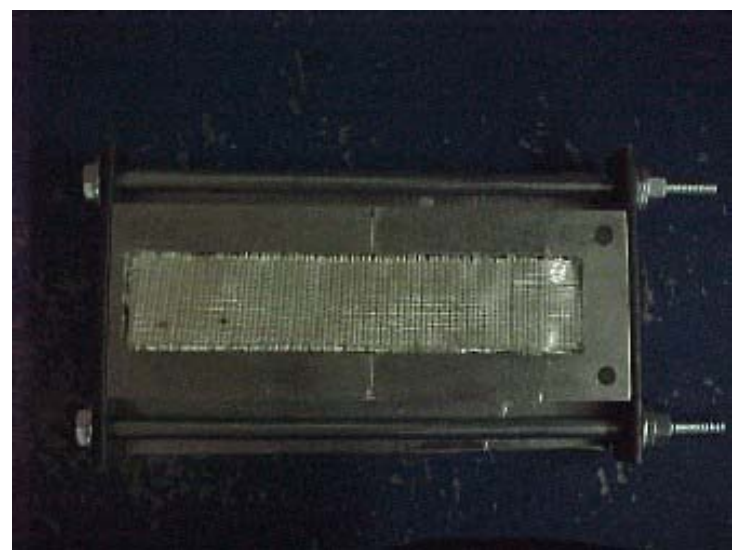

d) Glass Fabric on Inner Pellet Layer

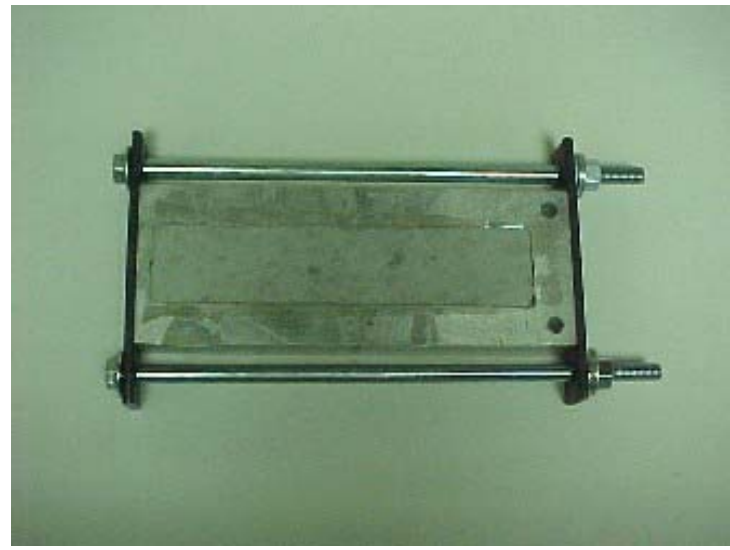

f) Molded Specimen in the Aluminum Apparatus

Figure 4.49 Compression Molding of Bond Specimen 

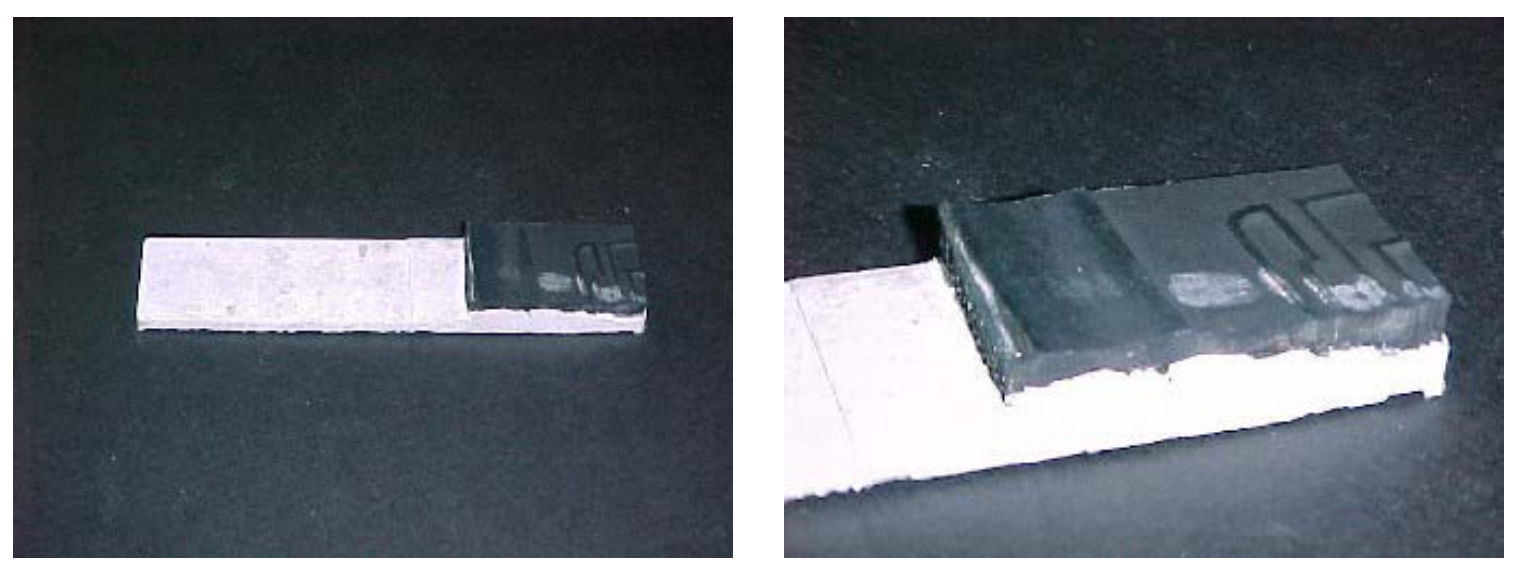

Figure 4.50 Rubber Tire-ABS Bond Specimen

\subsubsection{Specimen Preparation}

Excess resin from molding process on the ABS-rubber bond specimen was removed and edges were filed to prevent frictional resistance during pull out test. Inner walls of the mold were oiled to prevent undesired frictional resistance and the test sample was positioned in the mold (Fig. 4.51). The mold incidentally works as testing apparatus as well. Pulling grips were bolted on to the mold and attached with a hinged steel arm through a drilled hole on the ABS based specimen (Figure 4.51).
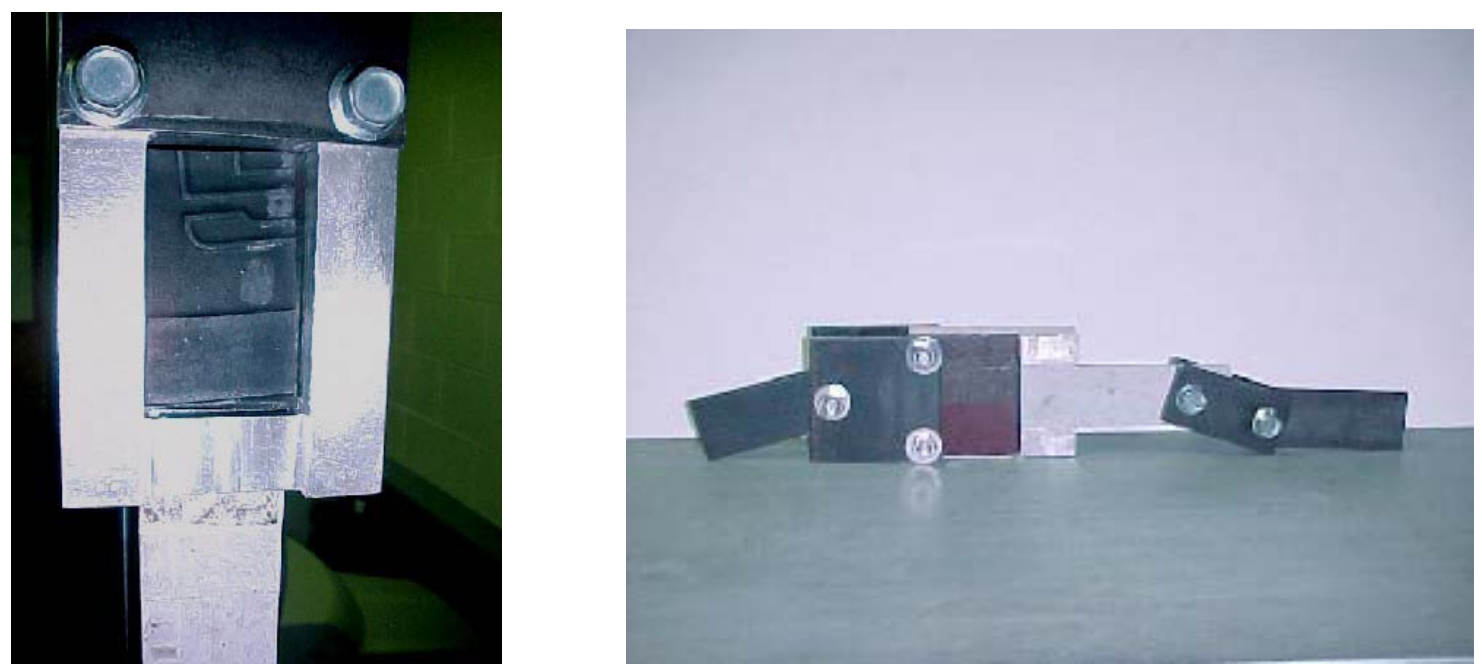

Figure 4.51 Bond Specimen Apparatus for Pull-out Test 


\subsubsection{Test Set-up and Procedure}

Instron 8500 two-column load frame-testing machine was used to conduct the bond pull-out test as shown in Figure 4.52. The test was computer controlled, including gathering of load and displacement data. Specimens were loaded at a constant rate of 300 lb/min until bond resistance dropped down to near zero and rubber block had de bonded from ABS or considerably deformed in addition to debonding.
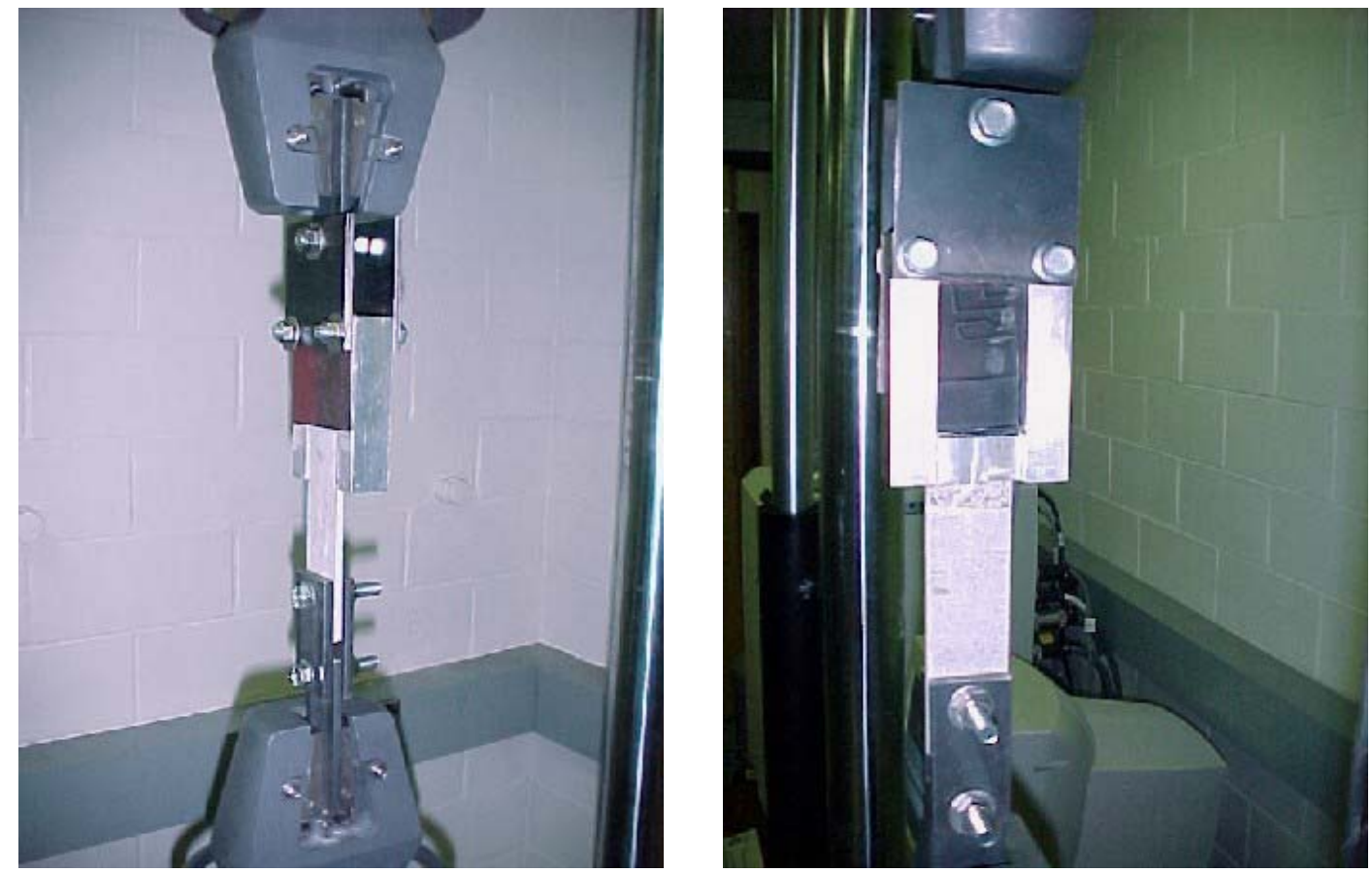

Figure 4.52 Bond Test Set-up

\subsection{Conclusions}

After trying different compression molding procedures and performing adequate modifications to the compression molding technique, an offset block for highway guardrails was successfully manufactured. Use of premolded tabs and preheat of offset block specimens prior to compression molding were necessary in order to obtain a satisfactory final product. Additional conclusions and recommendations are given in chapter 8 . 


\section{CHAPTER 5}

\section{TEST RESULTS, ANALYSIS AND DISCUSSIONS ON CONDITIONED RECYCLED COUPONS}

\subsection{Introduction}

As described in chapter 3, several static tests were performed on coupon specimens to determine their mechanical properties under tension, bending, compression, impact and hardness, for conditioned (aged) FRP specimens. In this section, test results are presented, discussed and compared to existing data from previous research (Bargo, 2000).

Characterization of aged coupon specimens will provide a better understanding about reduction in mechanical properties of the polymers due to environmental exposure. Based on the test results, it will be possible to determine knock down factors for tension, compression, bending, impact, and hardness that will be used for designing structural members.

\subsection{Tension Test}

\subsubsection{Results on ABS}

Tables 5.1, 5.2, F.1, and F.2 show strength and stiffness results for ABS specimens under tension. Maximum tensile strength and stiffness are provided for each type of ABS and PC specimens (virgin, 100\% recycled and virgin/recycled blend) and each aging period (2, 4, 10 and 18 months). Figures 5.1 and 5.2 show strength and stiffness variations, respectively, for different specimens and aging periods. 
Table 5.1 Tensile Strength Variations in Aged ABS Specimens

\begin{tabular}{|c|c|c|c|c|c|c|}
\hline \multirow{3}{*}{$\begin{array}{l}\text { Specimen } \\
\text { Type }\end{array}$} & \multicolumn{5}{|c|}{ Max. Tensile Stress (psi) } & $\%$ Change \\
\hline & \multicolumn{5}{|c|}{ Months of aging } & \multirow{2}{*}{$\begin{array}{c}\text { Maximum reduction in } \\
\text { period }\end{array}$} \\
\hline & $\mathbf{0}$ & 2 & 4 & 10 & 18 & \\
\hline $\mathrm{A} 1$ & 6174 & 5866 & - & 6118 & 5809 & -5.9 \\
\hline A2 & 10299 & 9683 & 10510 & 9410 & 10678 & -8.6 \\
\hline A3 & 5578 & 5285 & 5988 & 5549 & 5360 & -5.3 \\
\hline A4 & 8800 & 8802 & 9388 & 7602 & 8637 & -13.6 \\
\hline A5 & 5916 & 5558 & - & 5790 & 5683 & -6.1 \\
\hline A6 & 8911 & 9607 & 10619 & 9123 & 8916 & No reduction \\
\hline \multicolumn{6}{|c|}{ Average reduction without fibers (A1,A3,A5) } & -5.7 \\
\hline \multicolumn{6}{|c|}{ Average reduction with fibers (A2,A4,A6) } & -11.1 \\
\hline
\end{tabular}

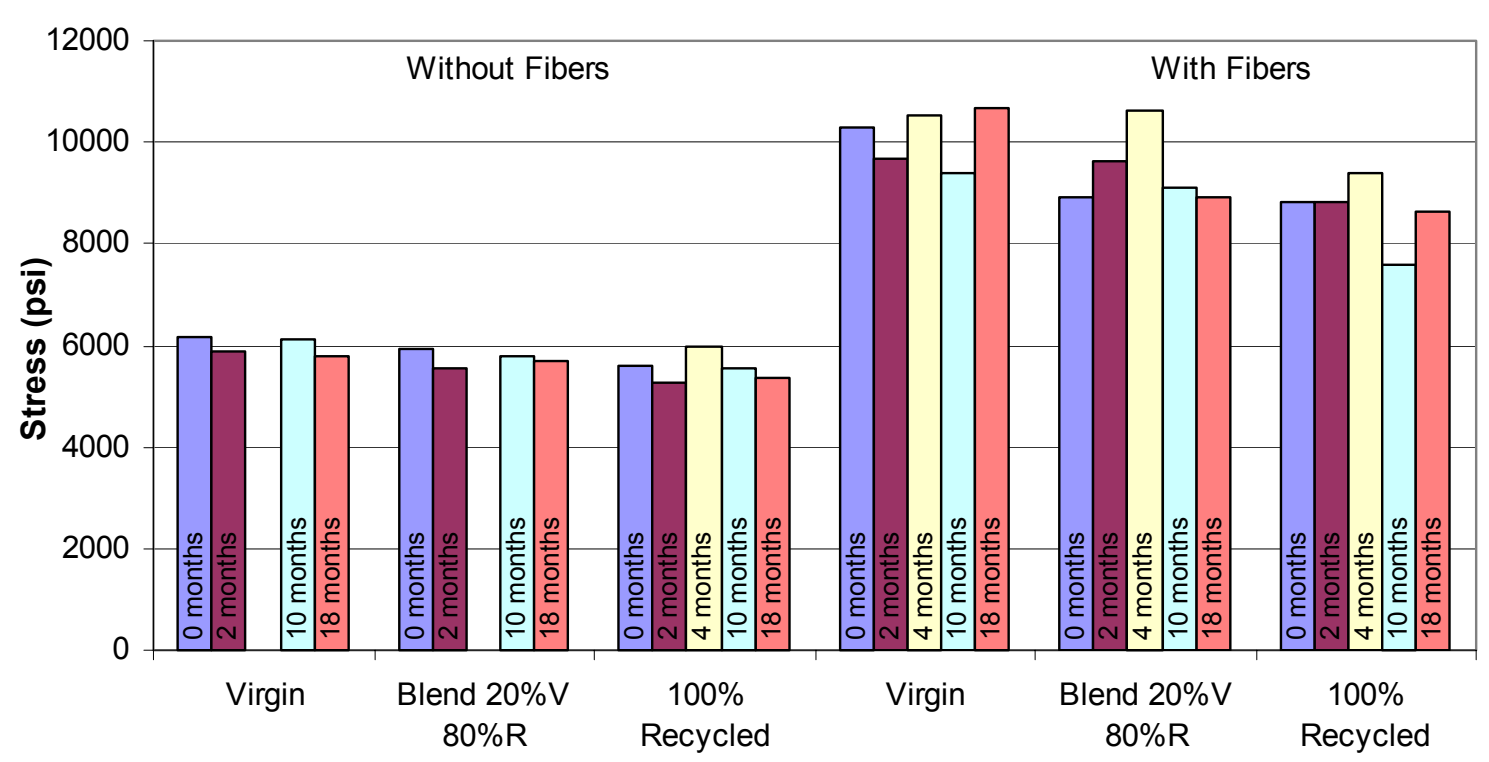

Note: Some intermittent results betw een 0 and 18 months may not be present due to no testing during that period

Figure 5.1 Tensile Strength in Aged ABS Specimens

Table 5.2 Tensile Stiffness Variations in Aged ABS Specimens

\begin{tabular}{|c|c|c|c|c|c|c|}
\hline \multirow{3}{*}{\begin{tabular}{|c|} 
Specimen \\
Type
\end{tabular}} & \multicolumn{5}{|c|}{ Tensile Stiffness (psi x10 ${ }^{6}$ ) } & \multirow{3}{*}{$\begin{array}{c}\text { \% Change } \\
\begin{array}{c}\text { Maximum reduction in } \\
\text { period }\end{array}\end{array}$} \\
\hline & \multicolumn{5}{|c|}{ Months of aging } & \\
\hline & $\mathbf{0}$ & 2 & 4 & 10 & 18 & \\
\hline A1 & 0.321 & - & 0.510 & 0.360 & 0.362 & No reduction \\
\hline A2 & 0.976 & - & 1.019 & 1.047 & 1.027 & No reduction \\
\hline A3 & 0.333 & - & - & 0.337 & 0.331 & -0.6 \\
\hline A4 & 0.964 & - & - & 0.909 & 1.012 & -5.7 \\
\hline A5 & 0.359 & - & 0.360 & 0.347 & 0.351 & -3.3 \\
\hline A6 & 0.941 & - & 1.050 & 0.993 & 1.041 & No reduction \\
\hline \multicolumn{6}{|c|}{ Average reduction without fibers (A1,A3,A5) } & -2.0 \\
\hline \multicolumn{6}{|c|}{ Average reduction with fibers $(\mathrm{A} 2, \mathrm{~A} 4, \mathrm{~A} 6)$} & -5.7 \\
\hline
\end{tabular}




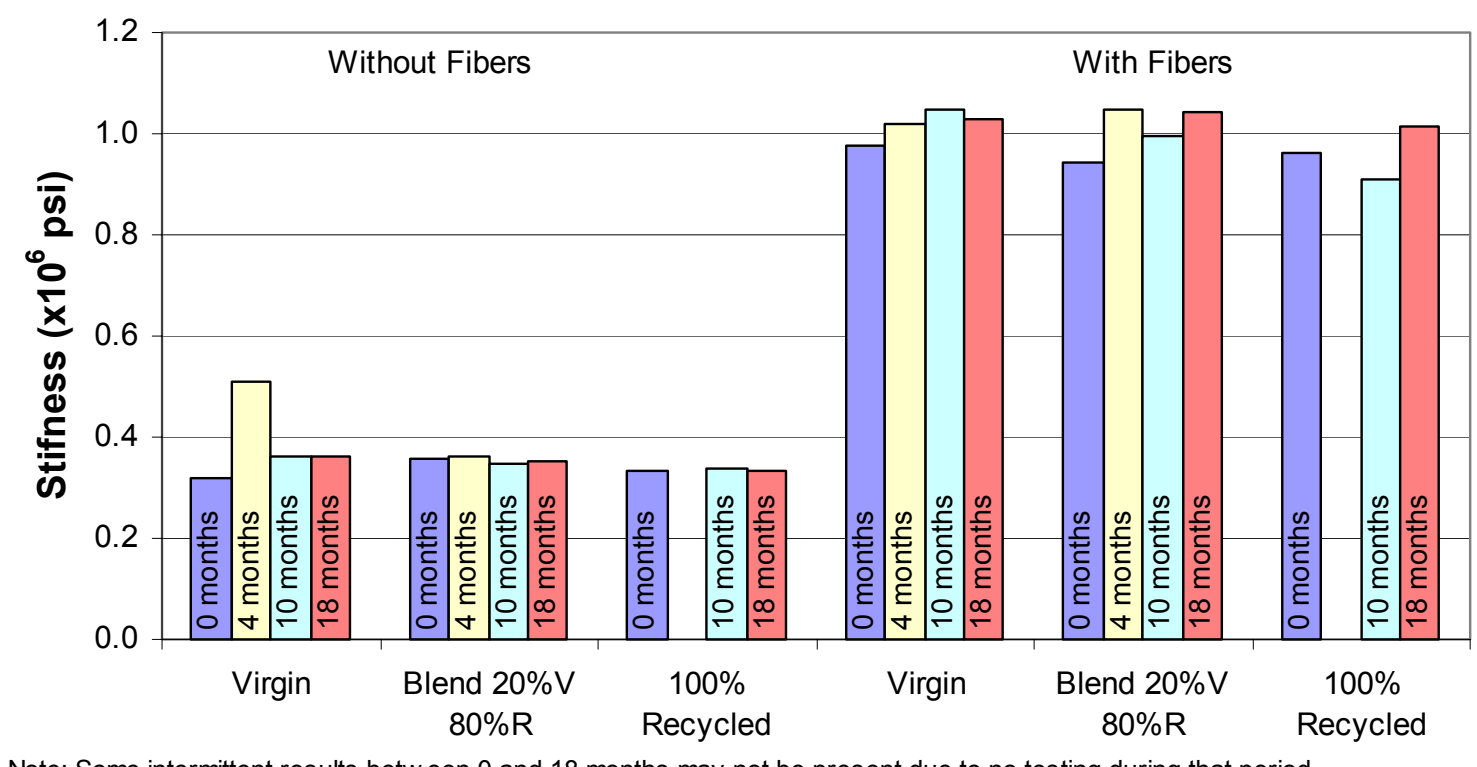

Note: Some intermittent results betw een 0 and 18 months may not be present due to no testing during that period

Figure 5.2 Tensile Stiffness in Aged ABS Specimens

\subsubsection{Analysis and Discussion on ABS}

Tables 5.1 and F.1 (in Appendix) show average reduction and average change in tensile strength for ABS samples.

Tensile Strength Variation in ABS at the End of the Aging Period:

- Reduction in tensile strength under conditioning varied from 3.9\% to 5.9\% for specimens without fibers and $1.9 \%$ to zero for specimens with fibers.

- Strength gain of $3.7 \%$ was observed in fiber reinforced specimen A2 having virgin resin.

- Average reduction in tensile strength after 18 months of conditioning for samples without fibers was $4.6 \%$ as compared to $1.9 \%$ in samples with fibers.

Tensile Strength Variation in ABS During the Aging Period:

- Maximum reductions were observed to range from $5.3 \%$ and $6.1 \%$ for specimens without fibers and 8.6 to $13.6 \%$ for specimens with fibers. 
- Specimen A3, blend of recycled and virgin resins, was the only unreinforced specimen that showed a gain in strength with a maximum of $7.4 \%$ at 4 months of aging.

- Reinforced specimens showed gains ranging from 3.7\% to $19.2 \%$. Average reduction during the aging period was $5.7 \%$ for specimens without fibers as compared to $11.1 \%$ for specimens with fibers.

Tables 5.2 and F.2 show the average reduction and average change in tensile stiffness for ABS samples.

Tensile Stiffness Variation in ABS at the End of the Aging Period:

- Reduction in tensile stiffness under conditioning varied from $0.6 \%$ to $2.2 \%$ for specimens without fibers and no reductions were observed for specimens with fibers.

- Stiffness gain of $12.8 \%$ was observed for unreinforced specimen A1 with virgin resin, whereas reinforced specimens showed gain of $5.0 \%$ to $10.6 \%$.

- After 18 months of conditioning, samples without fibers indicated a reduction of $1.4 \%$ and no reduction in specimens with fibers.

\section{Tensile Stiffness Variation in ABS During the Aging Period:}

- Reductions ranging from $0.6 \%$ to $3.3 \%$ for specimens without fibers, and $5.7 \%$ to zero for specimens with fibers were observed.

- Specimens without fibers showed a gain ranging from $0.3 \%$ to $58.9 \%$. Reinforced specimens showed maximum gain from $5.0 \%$ to $11.6 \%$.

- Average reduction during the aging period was $2.0 \%$ for specimens without fibers as compared to $5.7 \%$ for specimens with fibers. 
Gains observed in some of the specimens may be possibly due to secondary curing effects and improvement in force transfer across the resin/fiber interface. Additional comparisons to other mechanical properties are discussed in chapter 7.

\subsubsection{Results on PC}

Tables 5.3, 5.4, F.3, and F.4 show tensile strength and stiffness results for PC specimens. Maximum tensile strength and stiffness are provided for each type of specimen and each aging period. Figures 5.3 and 5.4 show maximum strength and stiffness, respectively, for each of the specimen types and aging periods..

Table 5.3 Tensile Strength Variations in Aged PC Specimens

\begin{tabular}{|c|c|c|c|c|c|c|}
\hline \multirow{2}{*}{$\begin{array}{c}\text { Specimen } \\
\text { Type }\end{array}$} & \multicolumn{5}{|c|}{ Max. Tensile Stress (psi) } & \% Change \\
\cline { 2 - 7 } & $\mathbf{7}$ & $\mathbf{2}$ & $\mathbf{4}$ & $\mathbf{1 0}$ & $\mathbf{1 8}$ & $\begin{array}{c}\text { Maximum reduction in } \\
\text { period }\end{array}$ \\
\cline { 2 - 7 } & $\mathbf{0}$ & 8210 & 9261 & 8817 & 8352 & -5.2 \\
\hline P1 & 8664 & 15101 & 15721 & 12867 & 11892 & -27.8 \\
\hline P2 & 16479 & 9233 & 8983 & 8329 & -8.8 \\
\hline P3 & 8566 & 7813 & 14205 & 12525 & 10920 & -28.2 \\
\hline P4 & 15213 & 14203 & 1420 & -9.6 \\
\hline P5 & 8926 & 8072 & 9163 & 8589 & 8185 & -28.4 \\
\hline P6 & 16555 & 14831 & - & 12877 & 11848 & -7.9 \\
\hline Average reduction without fibers (A1,A3,A5) \\
\hline Average reduction with fibers (A2,A4,A6) \\
\hline
\end{tabular}




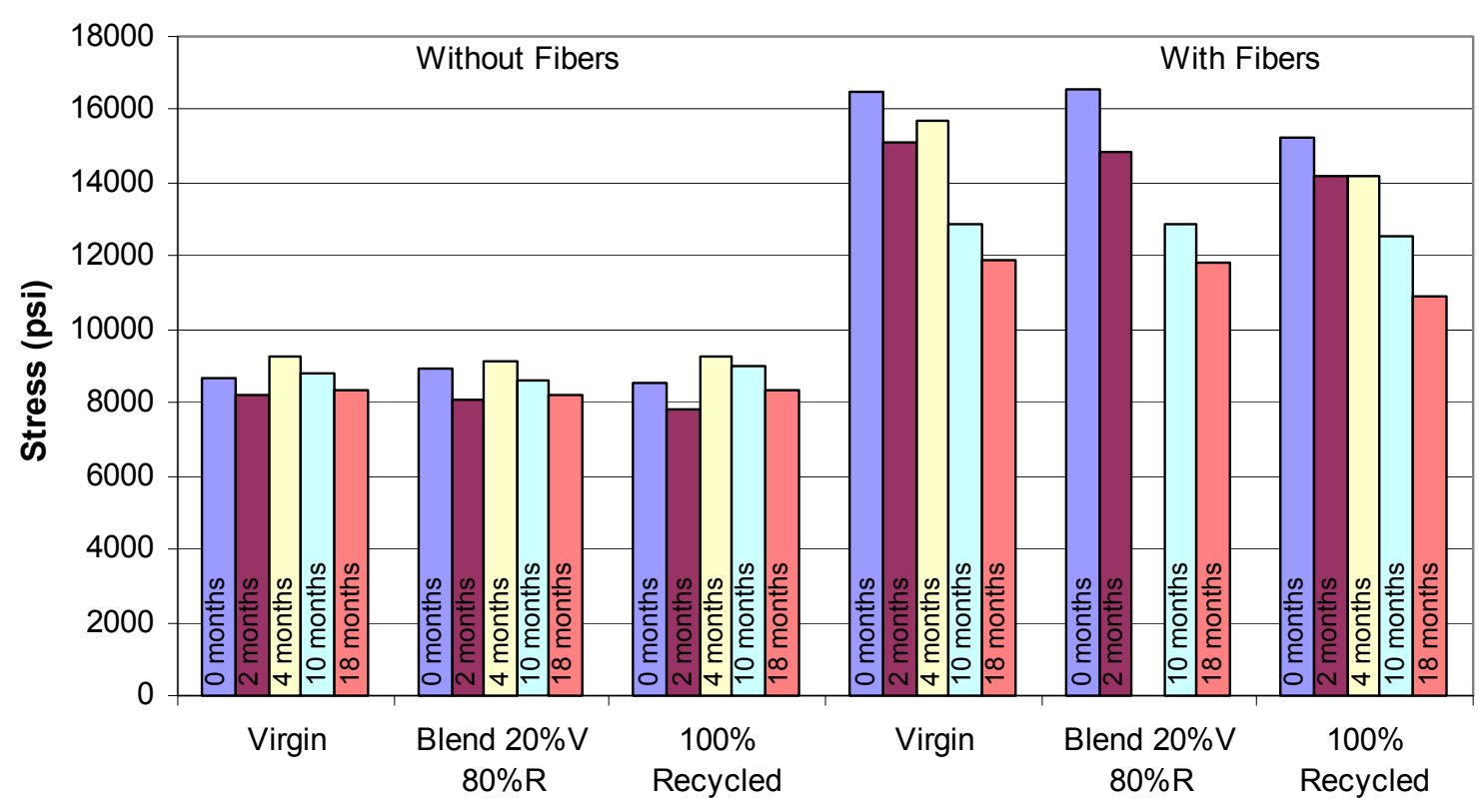

Note: Some intermittent results betw een 0 and 18 months may not be present due to no testing during that period

Figure 5.3 Tensile Strength in Aged PC Specimens

Table 5.4 Tensile Stiffness Variations in Aged PC Specimens

\begin{tabular}{|c|c|c|c|c|c|c|}
\hline \multirow{3}{*}{$\begin{array}{l}\text { Specimen } \\
\text { Type }\end{array}$} & \multicolumn{5}{|c|}{ Tension Stiffness (psi x10 ${ }^{6}$ ) } & \% Change \\
\hline & \multicolumn{5}{|c|}{ Months of aging } & \multirow{2}{*}{$\begin{array}{c}\text { Maximum reduction ir } \\
\text { period }\end{array}$} \\
\hline & $\mathbf{0}$ & 2 & 4 & 10 & 18 & \\
\hline $\mathrm{P} 1$ & 0.300 & - & 0.309 & 0.364 & 0.350 & No reduction \\
\hline P2 & 1.029 & - & 1.008 & 1.076 & 1.068 & -2.0 \\
\hline P3 & 0.293 & - & 0.315 & 0.364 & 0.343 & No reduction \\
\hline $\mathrm{P} 4$ & 0.993 & - & 0.964 & 1.061 & 1.073 & -2.9 \\
\hline P5 & 0.329 & - & 0.337 & 0.347 & 0.349 & No reduction \\
\hline P6 & 1.046 & - & - & 1.099 & 1.091 & No reduction \\
\hline \multicolumn{6}{|c|}{ Average reduction without fibers (A1,A3,A5) } & No reduction \\
\hline \multicolumn{6}{|c|}{ Average reduction with fibers $(\mathrm{A} 2, \mathrm{~A} 4, \mathrm{~A} 6)$} & -2.5 \\
\hline
\end{tabular}




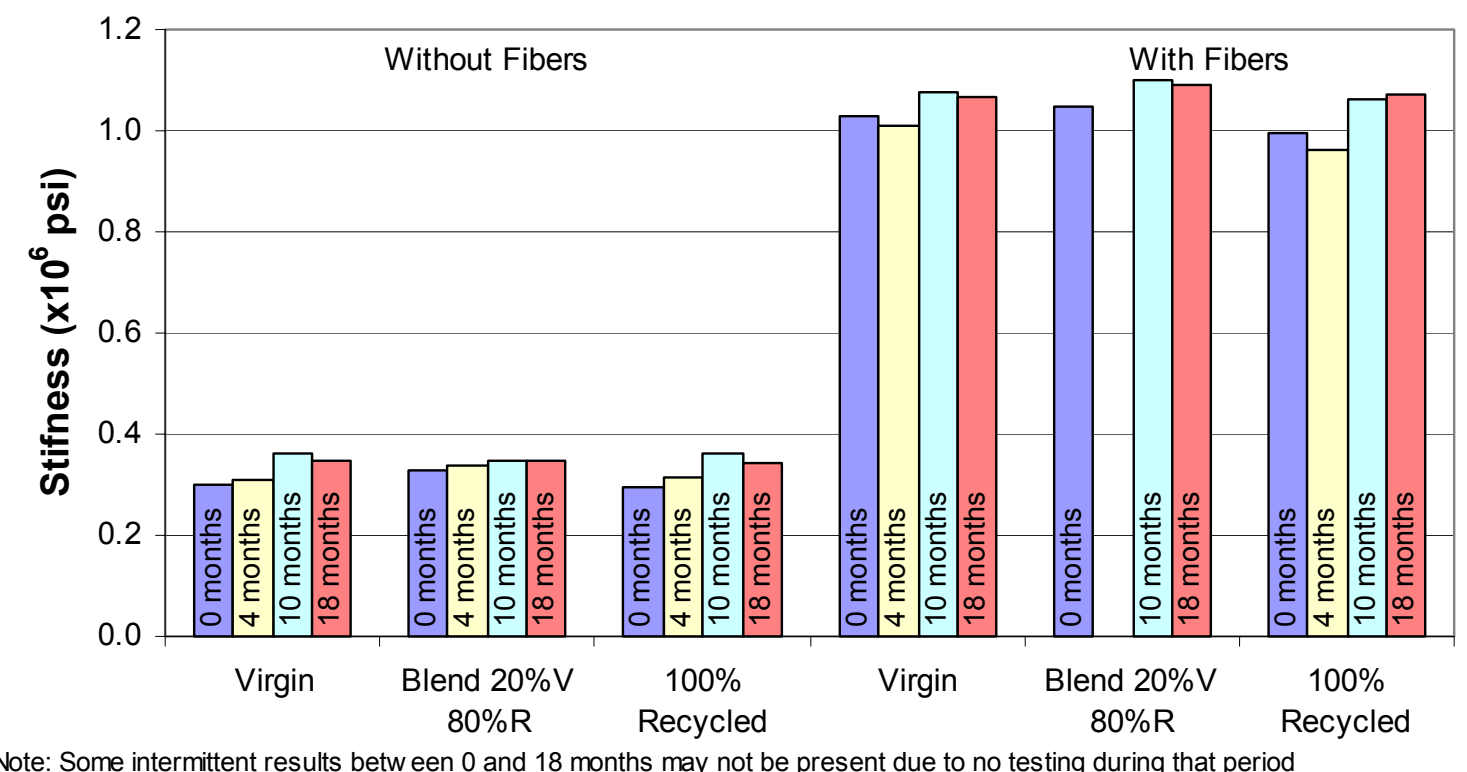

Figure 5.4 Tensile Stiffness in Aged PC Specimens

\subsubsection{Analysis and Discussion on PC}

Tables 5.3 and F.3 (Appendix) show the change in tensile strength for PC specimens.

\section{Tensile Strength Variation in PC at the End of the Aging Period}

- Reduction in tensile strength under conditioning varied from $2.8 \%$ to $8.3 \%$ for specimens without fibers and $27.8 \%$ to $28.4 \%$ for specimens with fibers.

- Average reduction in tensile strength after 18 months of conditioning for samples without fibers was $4.9 \%$ as compared to $28.2 \%$ in samples with fibers. 


\section{Tensile Strength Variation in PC During the Aging Period}

- Maximum tensile strength reductions between $5.2 \%$ and $9.6 \%$ for specimens without fibers and $27.8 \%$ to $28.4 \%$ for specimens with fibers were observed.

- Specimens without fibers showed maximum strength gains ranging from $2.7 \%$ to $7.8 \%$. No gain was observed for specimens without fibers.

- Maximum reduction due to aging was $7.9 \%$ for specimens without fibers as compared to $28.2 \%$ for specimens with fibers.

Tables 5.4 and F.4 show the average reduction in tensile stiffness for PC samples.

\section{Tensile Stiffness Variation in PC at the End of the Aging Period}

No reduction was noted in tensile stiffness under conditioned state for specimens without fibers or even for specimens with fibers.

\section{Tensile Stiffness Variation in PC During the Aging Period}

- No stiffness reductions for specimens without fibers were observed, whereas specimens with fibers showed maximum reductions of $2.0 \%$ to $2.9 \%$.

- Specimens without fibers showed maximum gain of $6.1 \%$ to $24.2 \%$ and specimens with fibers showed maximum gain of $4.6 \%$ to $8.1 \%$

- Maximum reduction during the aging period was $2.5 \%$ for specimens with fibers.

Gains observed in some of the specimens may be possibly due to secondary curing effects and improvement in force transfer across the resin/fiber interface. 


\subsection{Bending Test}

\subsubsection{Results on ABS}

Tables 5.5, 5.6, F.5, and F.6 show strength and stiffness results for aged ABS specimens under bending. Maximum bending strength and stiffness values are provided for each type of specimen and each aging period. Figures 5.5 and 5.6 show maximum strength and stiffness, respectively, for each of the specimen types and aging periods.

Table 5.5 Bending Strength Variations in Aged ABS Specimens

\begin{tabular}{|c|c|c|c|c|c|c|}
\hline \multirow{2}{*}{$\begin{array}{c}\text { Specimen } \\
\text { Type }\end{array}$} & \multicolumn{5}{|c|}{ Max. Bending Stress (psi) } & \% Change \\
\cline { 2 - 6 } & $\mathbf{0}$ & $\mathbf{2}$ & $\mathbf{4}$ & $\mathbf{1 0}$ & $\mathbf{1 8}$ & period \\
\cline { 2 - 7 } & $\mathbf{0}$ & - & 6728 & 10747 & 8971 & -25.9 \\
\hline A1 & 9080 & 16033 & 15211 & 14631 & 14080 & -20.8 \\
\hline A2 & 17771 & 8931 & 7862 & 8923 & 8511 & -14.4 \\
\hline A3 & 9187 & 15522 & 13886 & 12162 & 12605 & -27.3 \\
\hline A4 & 16740 & 9295 & 8412 & 10446 & 9419 & -12.0 \\
\hline A5 & 9558 & 16509 & -30.9 \\
\hline A6 & 16509 & 17793 & 14043 & 11400 & 13034 & -17.4 \\
\hline Average reduction without fibers (A1,A3,A5) \\
\hline
\end{tabular}

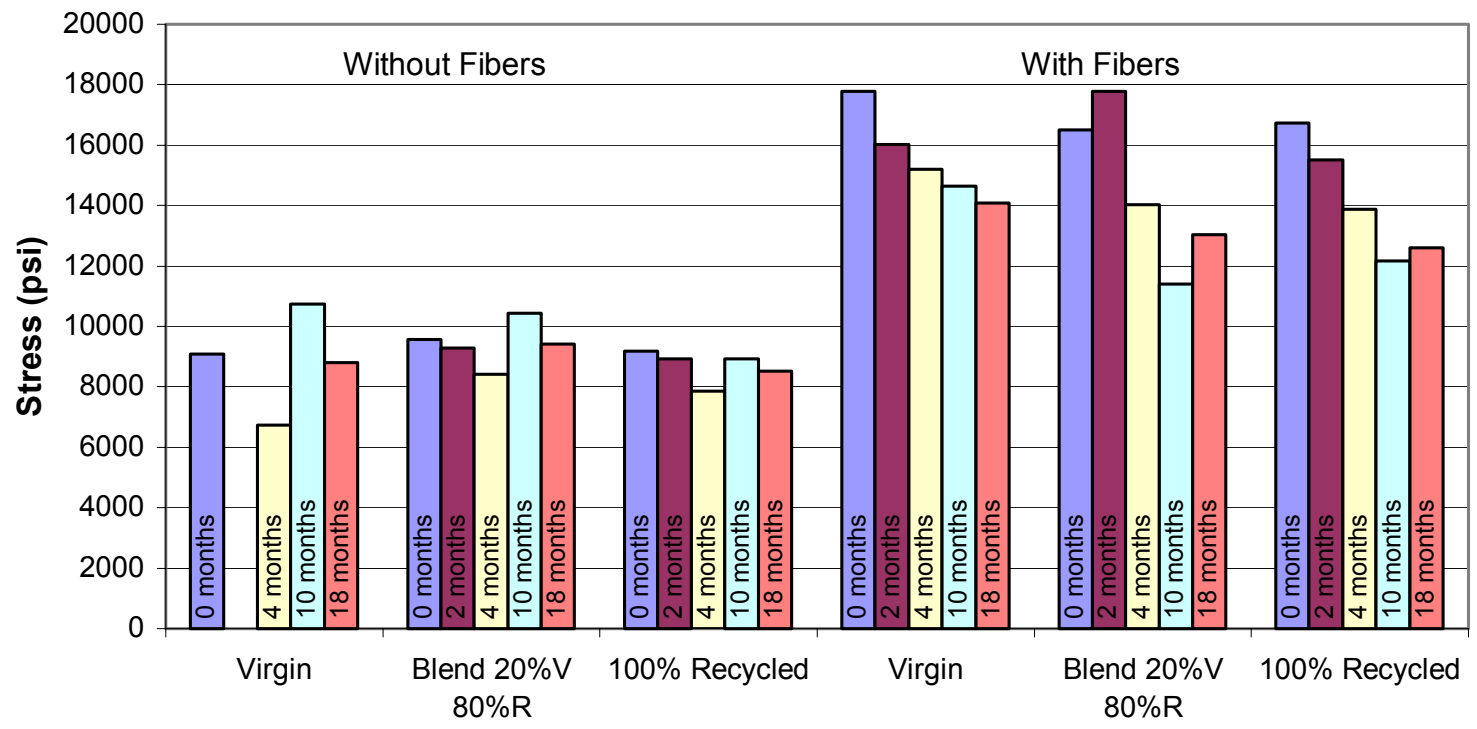

Note: Some intermittent results between 0 and 18 months may not be present due to no testing during that period

Figure 5.5 Bending Strength in Aged ABS Specimens 
Table 5.6 Bending Stiffness Variations in Aged ABS Specimens

\begin{tabular}{|c|c|c|c|c|c|c|}
\hline \multirow{2}{*}{$\begin{array}{c}\text { Specimen } \\
\text { Type }\end{array}$} & \multicolumn{5}{|c|}{ Bending Stiffness (psi x10 ${ }^{\mathbf{}}$ ) } & \% Change \\
\cline { 2 - 6 } & $\mathbf{7}$ & $\mathbf{2}$ & $\mathbf{4}$ & $\mathbf{1 0}$ & $\mathbf{1 8}$ & \\
\cline { 2 - 6 } & $\mathbf{0}$ & - & 0.334 & 0.699 & 0.381 & -16.5 \\
\hline A1 & 0.40 & - & 0.932 & 1.215 & 0.960 & -8.6 \\
\hline A2 & 1.02 & - & 0.364 & 0.408 & 0.391 & -9.0 \\
\hline A3 & 0.40 & - & 0.930 & 0.998 & 0.996 & -13.9 \\
\hline A4 & 1.08 & - & 0.346 & 0.385 & 0.395 & -13.5 \\
\hline A5 & 0.40 & - & 0.917 & 0.925 & 0.962 & -17.4 \\
\hline A6 & 1.11 & - & & -13.0 \\
\hline Average reduction without fibers (A1,A3,A5) \\
\hline Average reduction with fibers (A2,A4,A6) \\
\hline
\end{tabular}

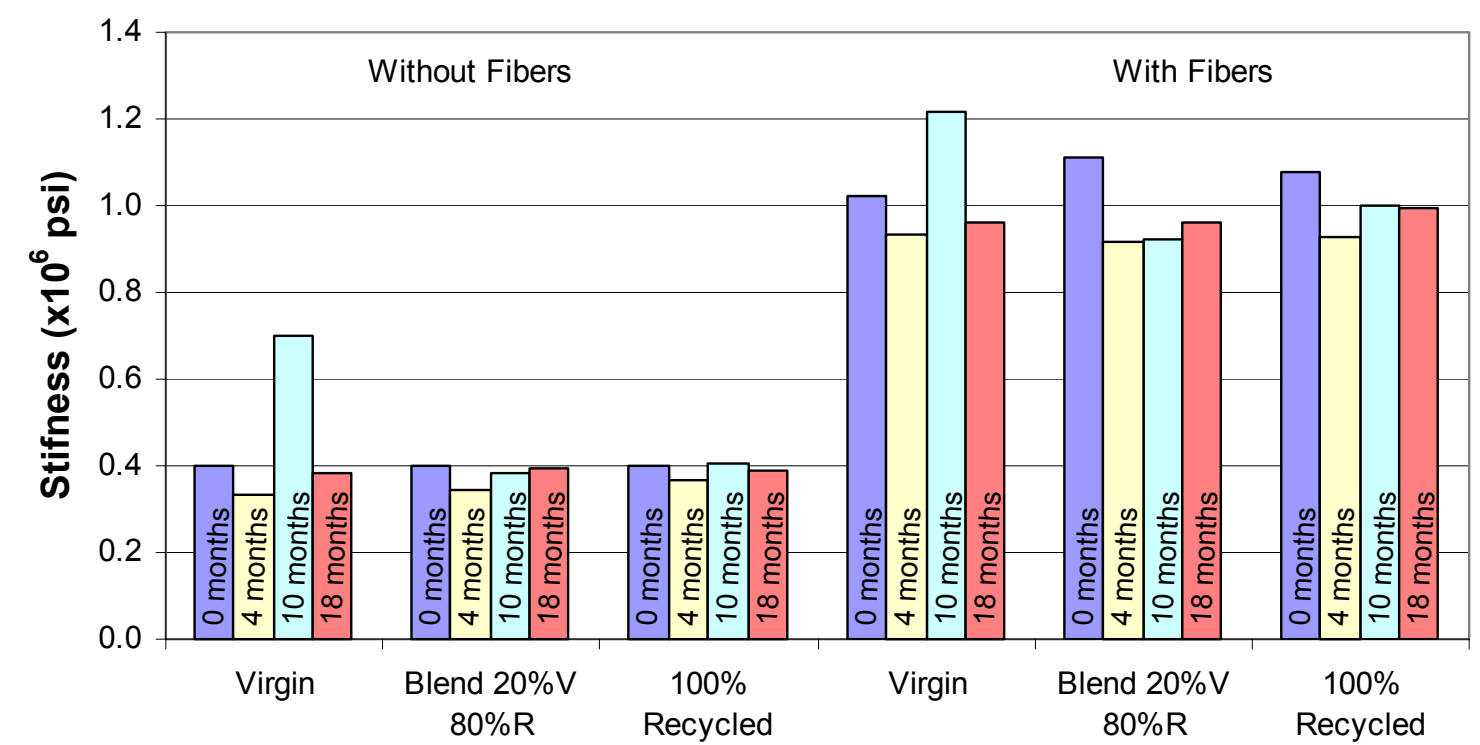

Note: Some intermittent results betw een 0 and 18 months may not be present due to no testing during that period

Figure 5.6 Bending Stiffness in Aged ABS Specimens

\subsubsection{Analysis and Discussion on ABS}

Tables 5.5 and F.5 shows the average reduction in bending strength for ABS samples. 


\section{Bending Strength Variation in ABS at the End of the Aging Period:}

- Reduction in bending strength under conditioning varied from $1.5 \%$ to $7.4 \%$ for specimens without fibers and $20.8 \%$ to $24.7 \%$ for specimens with fibers.

- Average reduction in bending strength after 18 months of conditioning for specimens without fibers was $4.0 \%$ as compared to $22.2 \%$ for samples with fibers.

\section{Bending Strength Variation in ABS During the Aging Period:}

- Maximum reductions were noted between $12.0 \%$ and $25.9 \%$ for specimens without fibers and $20.8 \%$ to $30.9 \%$ for specimens with fibers.

- Specimens without fibers showed maximum gains of $9.3 \%$ to $18.4 \%$.

- Average maximum reduction during the aging period was $17.4 \%$ for specimens without fibers as compared to $26.4 \%$ for specimens with fibers.

Tables 5.6 and F.6 show the average reduction in bending stiffness for ABS samples.

\section{Bending Stiffness Variation in ABS at the End of the Aging Period:}

- Reduction in bending stiffness is noted under conditioning which varied from $1.3 \%$ to $4.8 \%$ for specimens without fibers while the reductions were raging from $5.9 \%$ to $13.3 \%$ for specimens with fibers.

- Average reduction in bending stiffness after 18 months of conditioning for samples without fibers was $2.8 \%$ as compared to $9.0 \%$ for specimens with fibers. 


\section{Bending Stiffness Variation in ABS During the Aging Period:}

- Maximum stiffness reductions were noted between $9.0 \%$ and $16.5 \%$ for specimens without fibers and $8.6 \%$ to $17.4 \%$ for specimens with fibers.

- Specimens without fibers showed maximum stiffness gains of $2 \%$ to $74 \%$. Such high variation might be attributed to randomly oriented fibers in the specimens and possible manufacturing imperfections.

- Specimen A2 having virgin resin, was the only specimen with fibers that showed any gain, with a maximum of $19.1 \%$.

- Average maximum reduction during the aging period was $13.0 \%$ for specimens without fibers as compared to $13.3 \%$ for specimens with fibers.

Gains observed in some of the specimens may be possibly due to secondary curing effects and improvement in force transfer across the resin/fiber interface. Additional comparisons to other mechanical properties are discussed in chapter 7.

\subsubsection{Results on PC}

Tables 5.7, 5.8, F.7, and F.8 show strength and stiffness results for PC specimens tested. Maximum bending strength and stiffness are provided for each type of specimen and each aging period. Figures 5.7 and 5.8 show maximum strength and stiffness for each of the specimen types and aging periods, respectively. 
Table 5.7 Bending Strength Variations in Aged PC Specimens

\begin{tabular}{|c|c|c|c|c|c|c|}
\hline \multirow{2}{*}{$\begin{array}{c}\text { Specimen } \\
\text { Type }\end{array}$} & \multicolumn{5}{|c|}{ Max. Bending Stress (psi) } & \% Change \\
\cline { 2 - 6 } & \multicolumn{5}{|c|}{$\begin{array}{c}\text { Maximum reduction in } \\
\text { period }\end{array}$} \\
\cline { 2 - 5 } & $\mathbf{0}$ & $\mathbf{2}$ & $\mathbf{4}$ & $\mathbf{1 0}$ & $\mathbf{1 8}$ & -26.8 \\
\hline P1 & 10242 & 7494 & 9283 & 14945 & 12506 & -19.0 \\
\hline P2 & 24566 & 20228 & 21336 & 25370 & 19908 & -20.5 \\
\hline P3 & 10363 & 8235 & 8398 & 17327 & 12117 & -26.2 \\
\hline P4 & 23198 & 18830 & 17117 & 28586 & 19798 & -9.2 \\
\hline P5 & 11168 & 10136 & 10602 & 15023 & 12366 & -21.2 \\
\hline P6 & 24886 & 19606 & 21159 & 23909 & 20441 & -18.9 \\
\hline Average reduction without fibers (A1,A3,A5) & -22.1 \\
\hline Average reduction with fibers (A2,A4,A6) & & \\
\hline
\end{tabular}

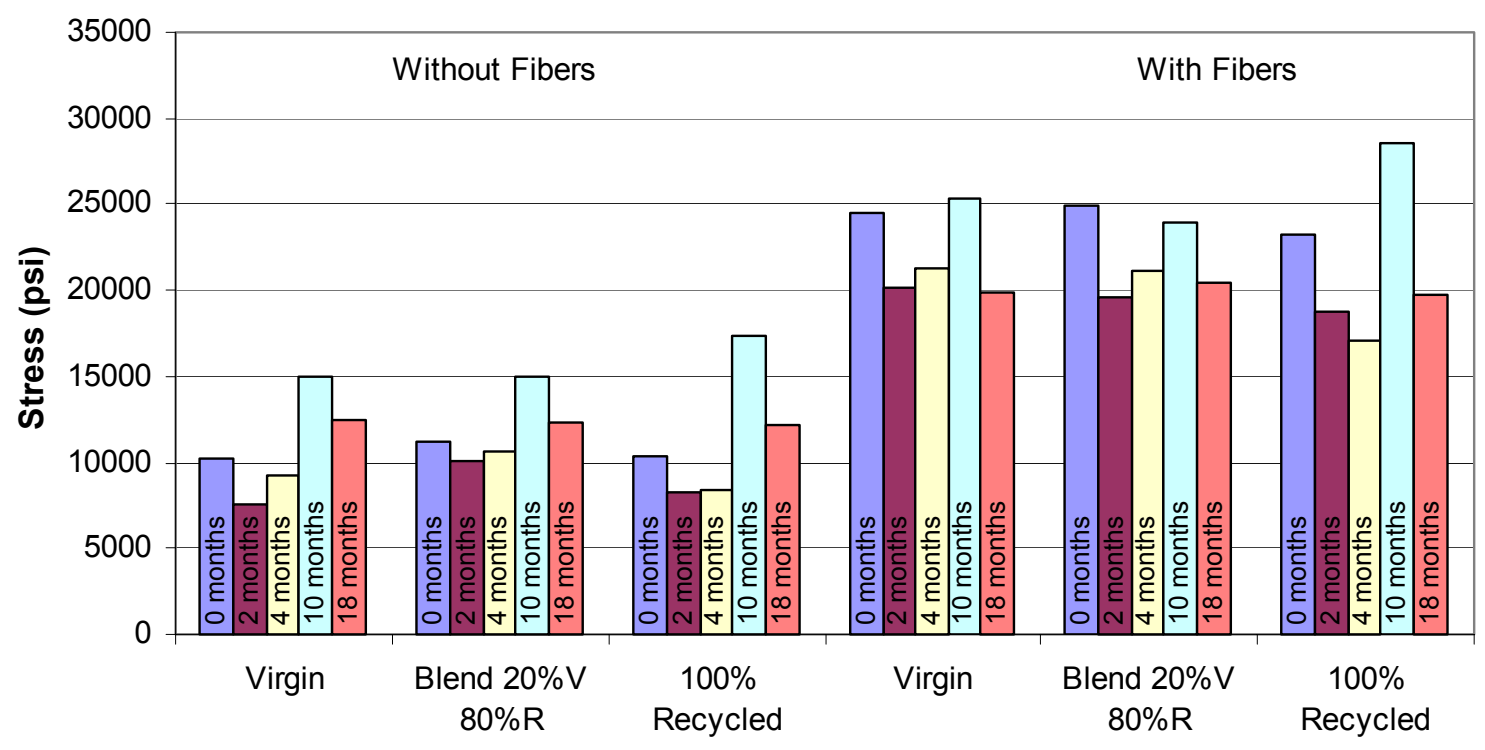

Figure 5.7 Bending Strength in Aged PC Specimens

Table 5.8 Bending Stiffness Variations in Aged PC Specimens

\begin{tabular}{|c|c|c|c|c|c|c|}
\hline \multirow{3}{*}{$\begin{array}{c}\text { Specimen } \\
\text { Type }\end{array}$} & \multicolumn{5}{|c|}{ Bending Stiffness $\left(p s i \times 10^{6}\right)$} & $\%$ Change \\
\hline & \multicolumn{5}{|c|}{ Months of aging } & \multirow{2}{*}{$\begin{array}{c}\text { Maximum reduction in } \\
\text { period }\end{array}$} \\
\hline & $\mathbf{0}$ & 2 & 4 & 10 & 18 & \\
\hline P1 & 0.332 & - & 0.341 & 0.416 & 0.408 & No reduction \\
\hline $\mathrm{P} 2$ & 0.967 & - & 1.001 & 1.089 & 1.031 & No reduction \\
\hline $\mathrm{P} 3$ & 0.291 & - & 0.354 & 0.445 & 0.450 & No reduction \\
\hline P4 & 0.916 & - & 0.994 & 1.001 & 1.037 & No reduction \\
\hline P5 & 0.327 & - & 0.406 & 0.452 & 0.430 & No reduction \\
\hline P6 & 0.993 & - & 1.062 & 1.168 & 1.027 & No reduction \\
\hline \multicolumn{6}{|c|}{ Average reduction without fibers (A1,A3,A5) } & No reduction \\
\hline \multicolumn{6}{|c|}{ Average reduction with fibers $(\mathrm{A} 2, \mathrm{~A} 4, \mathrm{~A} 6)$} & No reduction \\
\hline
\end{tabular}




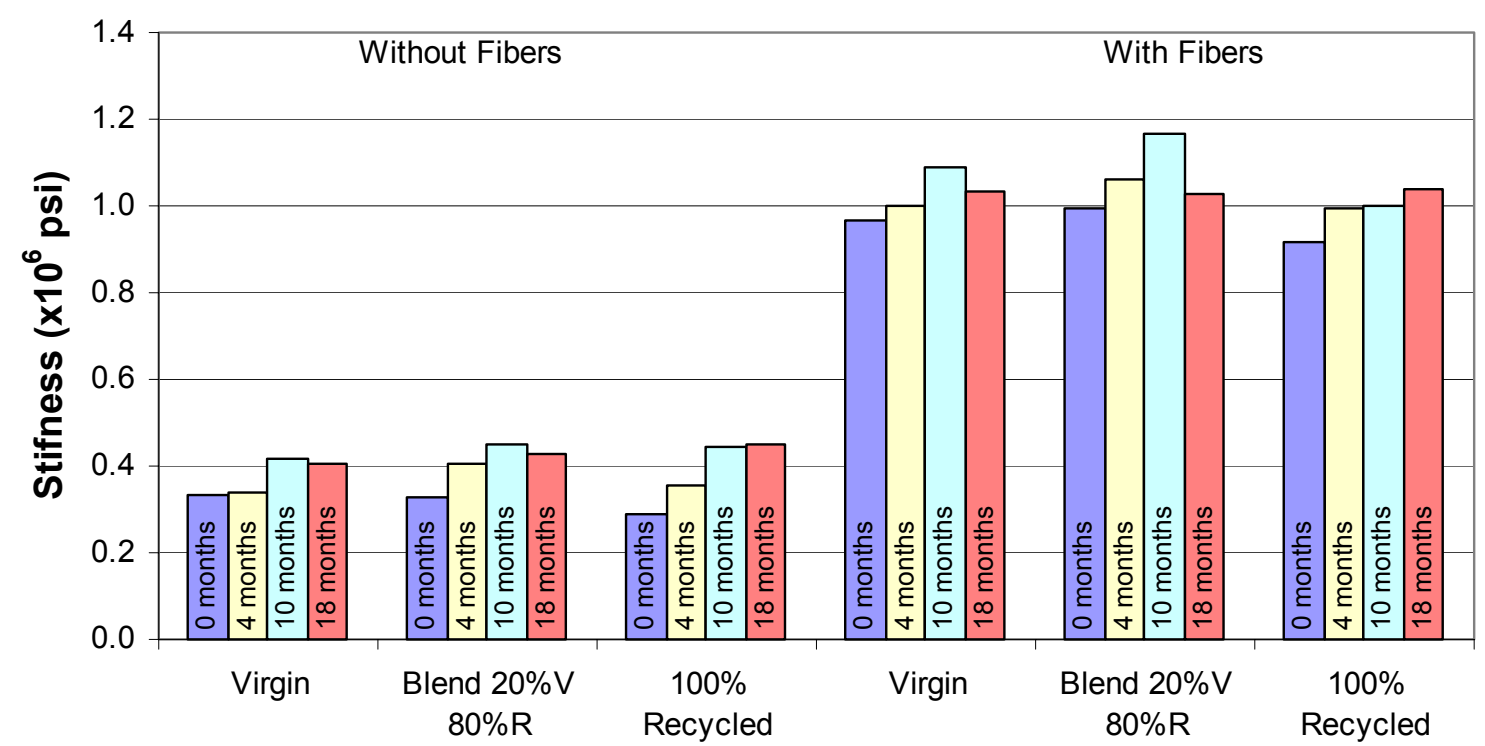

Note: Some intermittent results betw een 0 and 18 months may not be present due to no testing during that period

Figure 5.8 Bending Stiffness in Aged PC Specimens

\subsubsection{Analysis and Discussion on PC}

Table 5.7and F.7 shows the average reduction in bending strength for PC samples.

\section{Bending Strength Variation in PC at the End of the Aging Period:}

- No reduction in bending strength was noted under conditioning for specimens without fibers, whereas $14.7 \%$ to $19.0 \%$ reduction was obtained for specimens with fibers.

- Average reduction of $17.2 \%$ was noted in bending strength after 18 months of conditioning for samples with fibers.

\section{Bending Strength Variation in PC During the Aging Period:}

- Maximum bending strength reductions ranging between $9.2 \%$ and $26.8 \%$ were observed for specimens without fibers and $19.0 \%$ to $26.2 \%$ for specimens with fibers. 
- Specimens without fibers showed maximum gains of $34.5 \%$ to $67.2 \%$, whereas specimens with fibers showed maximum gains of $3.3 \%$ to $23.2 \%$.

- Maximum average bending strength reduction during the aging period was $18.9 \%$ for specimens without fibers as compared to $22.1 \%$ for specimens with fibers.

Tables 5.8 and F.7 show the average reduction in bending stiffness for PC samples.

Bending Stiffness Variation in PC at the End of the Aging Period:

- No reductions in bending stiffness were observed under conditioning for specimens both without and with fibers.

- Stiffness gain ranging between $22.9 \%$ to $54.6 \%$ was observed for specimens without fibers and $3.4 \%$ to $13.2 \%$ for specimens with fibers.

\section{Bending Stiffness Variation in PC During the Aging Period:}

No reductions in bending stiffness were observed during the entire period of aging, whereas gains of $25.3 \%$ to $54.6 \%$ was observed for specimens without fibers and $12.6 \%$ to $17.6 \%$ for specimens with fibers.

Gains observed in some of the specimens may be possibly due to secondary curing effects and improvement in force transfer across the resin/fiber interface. However, it should be noted that the bending strength gains after 10 months of conditioning are high and the tests may have to be repeated to ascertain such high gains 


\subsection{Compression Test}

\subsubsection{Results on ABS}

Tables 5.9, 5.10, F.9, and F.10 show strength and stiffness results for ABS specimens tested. Maximum compressive strength and stiffness are provided for each type of specimen and each aging period. Figures 5.9 and 5.10 show maximum strength and stiffness for each of the specimen types and aging periods, respectively.

Table 5.9 Compressive Strength Variations in Aged ABS Specimens

\begin{tabular}{|c|c|c|c|c|c|c|}
\hline \multirow{2}{*}{$\begin{array}{c}\text { Specimen } \\
\text { Type }\end{array}$} & \multicolumn{5}{|c|}{ Max. Compressive Stress (psi) } & \% Change \\
\cline { 2 - 6 } & $\mathbf{7}$ & $\mathbf{2}$ & $\mathbf{4}$ & $\mathbf{1 0}$ & $\mathbf{1 8}$ & $\begin{array}{c}\text { Maximum reduction in } \\
\text { period }\end{array}$ \\
\cline { 2 - 6 } & $\mathbf{0}$ & - & 11698 & - & 10376 & -8.1 \\
\hline A1 & 8966 & 9208 & 9886 & - & 8244 & -21.0 \\
\hline A2 & 13135 & - & 8670 & - & 8381 & -8.7 \\
\hline A3 & 8792 & 8027 & 12440 & - & 10120 & -32.4 \\
\hline A4 & 14891 & 10061 & 120 & -7.3 \\
\hline A5 & 8972 & 8318 & 9287 & - & 9011 & -25.9 \\
\hline A6 & 13920 & 10308 & 11151 & - & 11265 & -8.0 \\
\hline Average reduction without fibers (A1,A3,A5) \\
\hline Average reduction with fibers (A2,A4,A6) \\
\hline
\end{tabular}

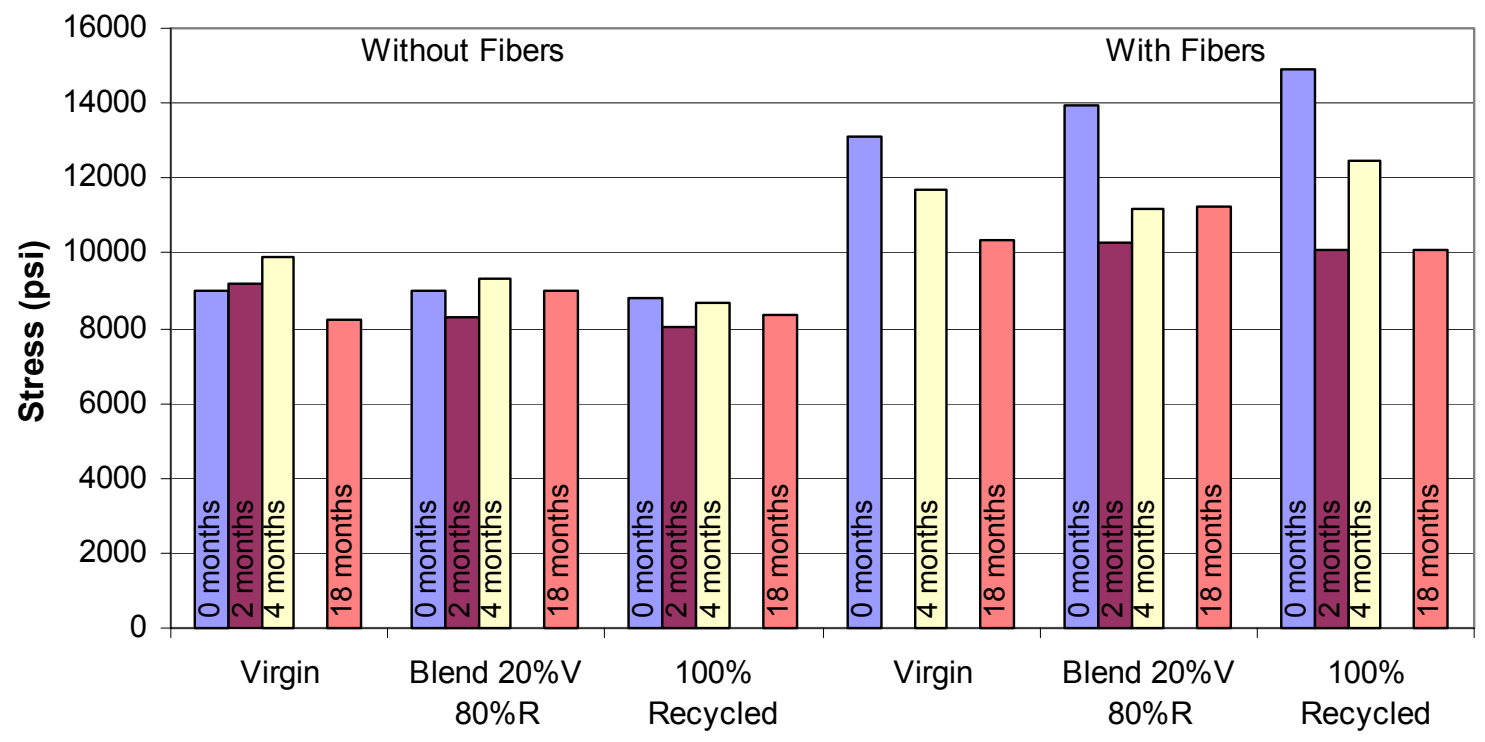

Note: Some intermittent results betw een 0 and 18 months may not be present due to no testing during that period

Figure 5.9 Compressive Strength in Aged ABS Specimens 
Table 5.10 Compressive Stiffness Variations in Aged ABS Specimens

\begin{tabular}{|c|c|c|c|c|c|c|}
\hline \multirow{3}{*}{$\begin{array}{c}\text { Specimen } \\
\text { Type }\end{array}$} & \multicolumn{5}{|c|}{ Compressive Stiffness (psi) } & \multirow{3}{*}{\begin{tabular}{|c|}
$\%$ Change \\
$\begin{array}{c}\text { Maximum reduction in } \\
\text { period }\end{array}$
\end{tabular}} \\
\hline & \multicolumn{5}{|c|}{ Months of aging } & \\
\hline & $\mathbf{0}$ & 2 & 4 & $\mathbf{1 0}$ & 18 & \\
\hline$\overline{\mathrm{A} 1}$ & 0.34 & - & - & - & 0.397 & No reduction \\
\hline A2 & 0.91 & - & - & - & 0.943 & No reduction \\
\hline $\mathrm{A} 3$ & 0.42 & - & - & - & 0.583 & No reduction \\
\hline A4 & 0.92 & - & - & - & 0.600 & -34.8 \\
\hline A5 & 0.41 & - & - & - & 0.330 & -19.5 \\
\hline A6 & 1.02 & - & - & - & 0.709 & -30.5 \\
\hline \multicolumn{6}{|c|}{ Average reduction without fibers $(\mathrm{A} 1, \mathrm{~A} 3, \mathrm{~A} 5)$} & -19.5 \\
\hline \multicolumn{6}{|c|}{ Average reduction with fibers $(\mathrm{A} 2, \mathrm{~A} 4, \mathrm{~A} 6)$} & -32.6 \\
\hline
\end{tabular}

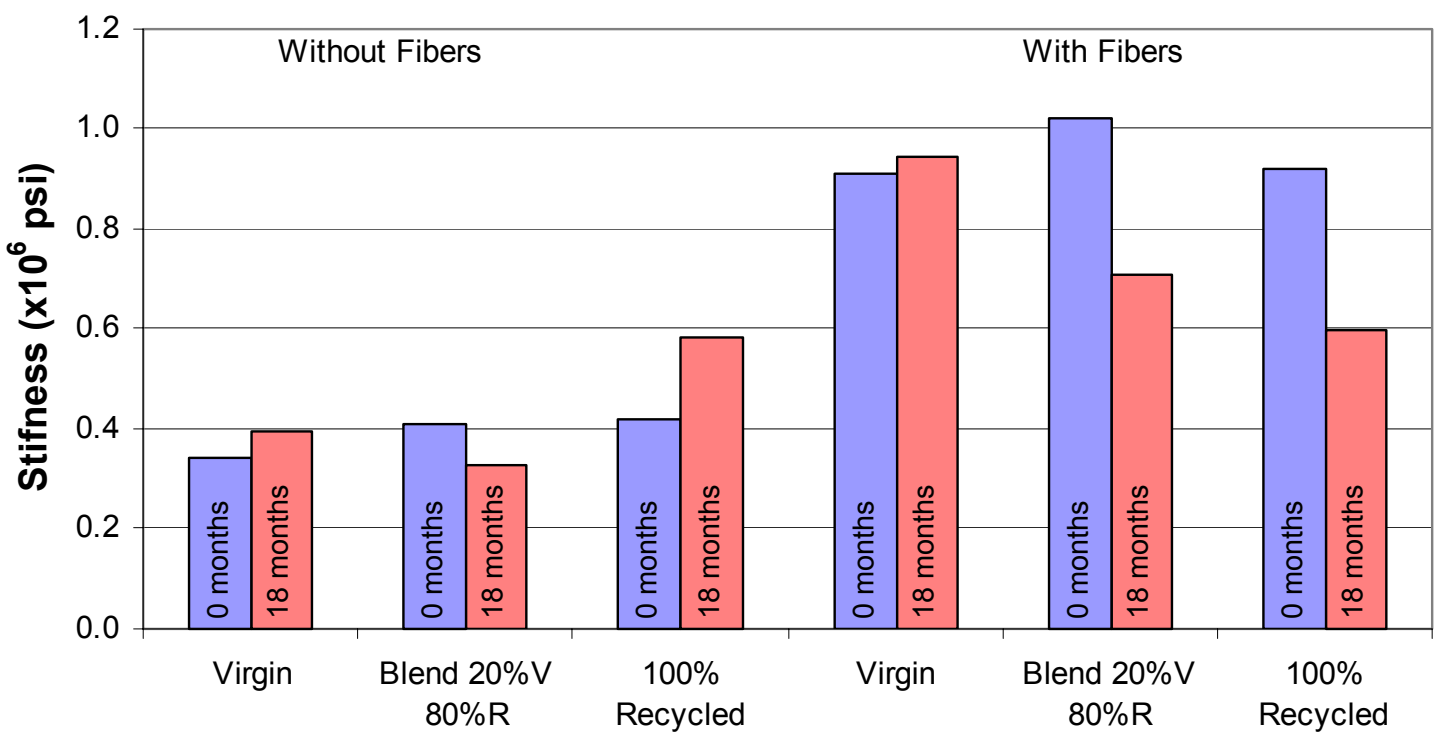

Note: Some intermittent results betw een 0 and 18 months may not be present due to no testing during that period

Figure 5.10 Compressive Stiffness in Aged ABS Specimens

\subsubsection{Analysis and Discussion on ABS}

Tables 5.9 and F.9 show average reduction in compressive strength for ABS samples. 


\section{Compressive Strength Variation in ABS at the End of the Aging Period:}

- Reduction in compressive strength under conditioning varied from $4.7 \%$ to $8.1 \%$ for specimens without fibers and $19.1 \%$ to $32.0 \%$ for specimens with fibers.

- Average reduction in compressive strength after 18 months of conditioning for samples without fibers was $6.4 \%$ as compared to $24.0 \%$ in samples with fibers.

\section{Compressive Strength Variation in ABS During the Aging Period:}

- Maximum reductions were noted to range from $7.3 \%$ to $8.7 \%$ for specimens without fibers and $21.0 \%$ to $32.4 \%$ for specimens with fibers.

- Specimens without fibers showed maximum gains of $3.5 \%$ to $10.3 \%$, whereas no gain was observed for specimens with fibers.

- Maximum average reduction during the aging period was $8.0 \%$ for specimens without fibers as compared to $26.5 \%$ for specimens with fibers.

Tables 5.10 and F.10 show the average reduction in compressive stiffness for ABS samples.

\section{Compressive Stiffness Variation in ABS at the End of the Aging Period:}

- Reduction in compressive stiffness under conditioning varied from $19.8 \%$ to zero for specimens without fibers whereas reductions of $30.5 \%$ to $34.8 \%$ were observed for specimens with fibers. 
- Average reduction in bending stiffness after 18 months of conditioning of specimens without fibers was $19.8 \%$ as compared to $32.6 \%$ for specimens with fibers.

- Average gain in maximum compressive stiffness observed for specimens without fibers was $27.8 \%$ and $3.6 \%$ for specimens with fibers.

Gains observed in some of the specimens may be possibly due to secondary curing effects and improvement in force transfer across the resin/fiber interface.

It should be noted that the compressive strength was not measured after 10 months of conditioning and tests may have to be conducted to obtain further results.

\subsubsection{Results on PC}

Tables 5.11, 5.12, F.11, and F.12 show strength and stiffness results for PC specimens. Maximum compressive strength and stiffness are provided for each type of specimen and aging period. Figures 5.11 and 5.12 show maximum strength and stiffness, respectively, for different specimen types and aging period.

Table 5.11 Compressive Strength Variations in Aged PC Specimens

\begin{tabular}{|c|c|c|c|c|c|c|}
\hline \multirow{3}{*}{$\begin{array}{c}\text { Specimen } \\
\text { Type }\end{array}$} & \multicolumn{5}{|c|}{ Max. Compressive Stress (psi) } & $\%$ Change \\
\hline & \multicolumn{5}{|c|}{ Months of aging } & \multirow{2}{*}{$\begin{array}{c}\text { Maximum reduction in } \\
\text { period }\end{array}$} \\
\hline & $\mathbf{0}$ & 2 & 4 & $\mathbf{1 0}$ & 18 & \\
\hline P1 & 10423 & 10234 & 10259 & - & 10344 & -1.8 \\
\hline $\mathrm{P} 2$ & 20332 & 12408 & 13539 & - & 12587 & -39.0 \\
\hline P3 & 10311 & 9894 & 11348 & - & 10296 & -4.0 \\
\hline $\mathrm{P} 4$ & 20747 & 11463 & 13509 & - & 13749 & -44.7 \\
\hline P5 & 10523 & 9931 & 10979 & - & 10221 & -5.6 \\
\hline P6 & 21124 & 12412 & 17500 & - & 12422 & -41.2 \\
\hline \multicolumn{6}{|c|}{ Average reduction without fibers (A1,A3,A5) } & -3.8 \\
\hline \multicolumn{6}{|c|}{ Average reduction with fibers $(\mathrm{A} 2, \mathrm{~A} 4, \mathrm{~A} 6)$} & -41.7 \\
\hline
\end{tabular}




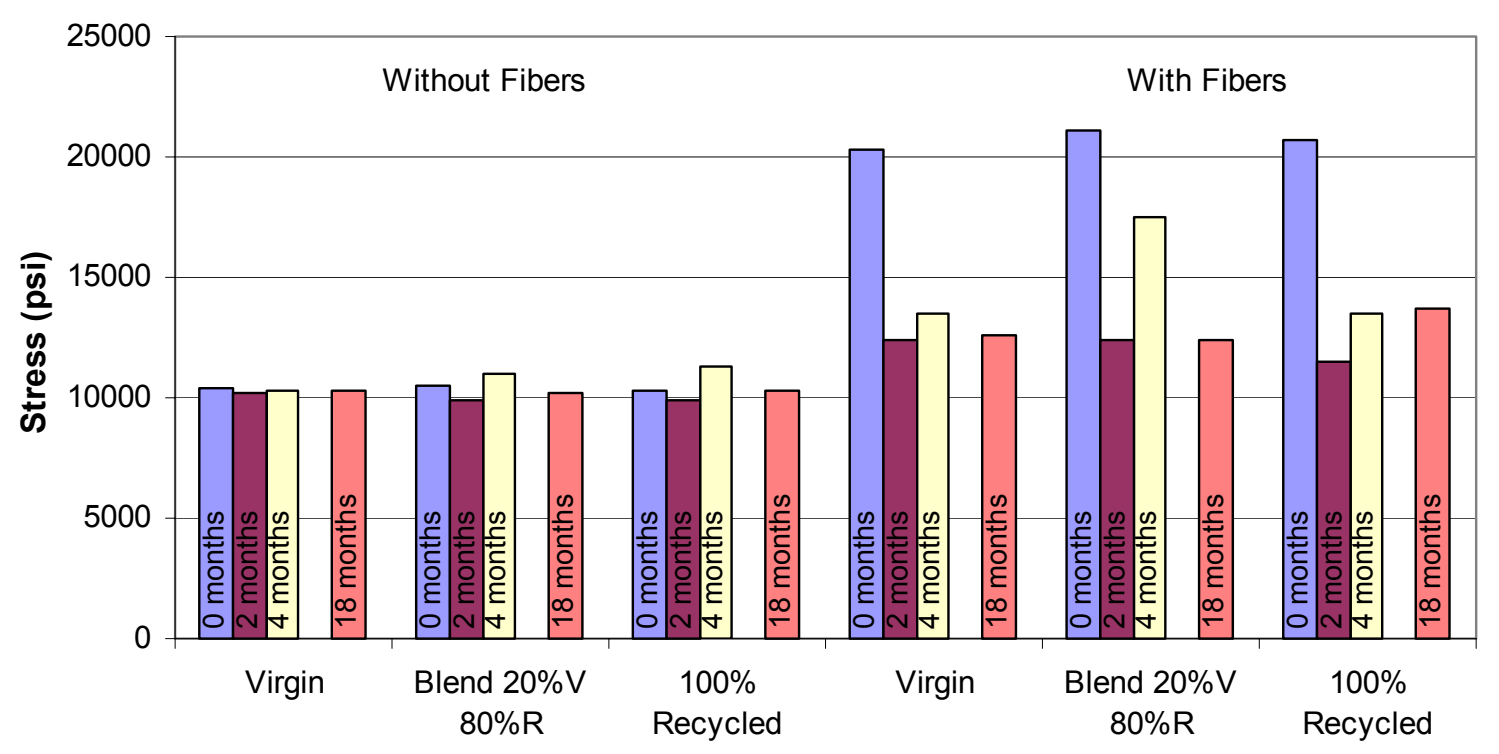

Note: Some intermittent results betw een 0 and 18 months may not be present due to no testing during that period

Figure 5.11 Compressive Strength in Aged PC Specimens

Table 5.12 Compressive Stiffness Variations in Aged PC Specimens

\begin{tabular}{|c|c|c|c|c|c|c|}
\hline \multirow{3}{*}{$\begin{array}{c}\text { Specimen } \\
\text { Type }\end{array}$} & \multicolumn{5}{|c|}{ Compressive Stiffness (psi) } & \% Change \\
\hline & \multicolumn{5}{|c|}{ Months of aging } & \multirow{2}{*}{$\begin{array}{c}\text { Maximum reduction in } \\
\text { period }\end{array}$} \\
\hline & $\mathbf{0}$ & 2 & 4 & $\mathbf{1 0}$ & 18 & \\
\hline P1 & 0.21 & - & 0.283 & - & 0.320 & No reduction \\
\hline P2 & 0.41 & - & 0.481 & - & 0.511 & No reduction \\
\hline P3 & 0.20 & - & 0.302 & - & 0.344 & No reduction \\
\hline P4 & 0.43 & - & 0.463 & - & 0.494 & No reduction \\
\hline P5 & 0.21 & - & 0.355 & - & - & No reduction \\
\hline P6 & 0.43 & - & 0.481 & - & 0.477 & No reduction \\
\hline \multicolumn{6}{|c|}{ Average reduction without fibers (A1,A3,A5) } & No reduction \\
\hline \multicolumn{6}{|c|}{ Average reduction with fibers $(\mathrm{A} 2, \mathrm{~A} 4, \mathrm{~A} 6)$} & No reduction \\
\hline
\end{tabular}




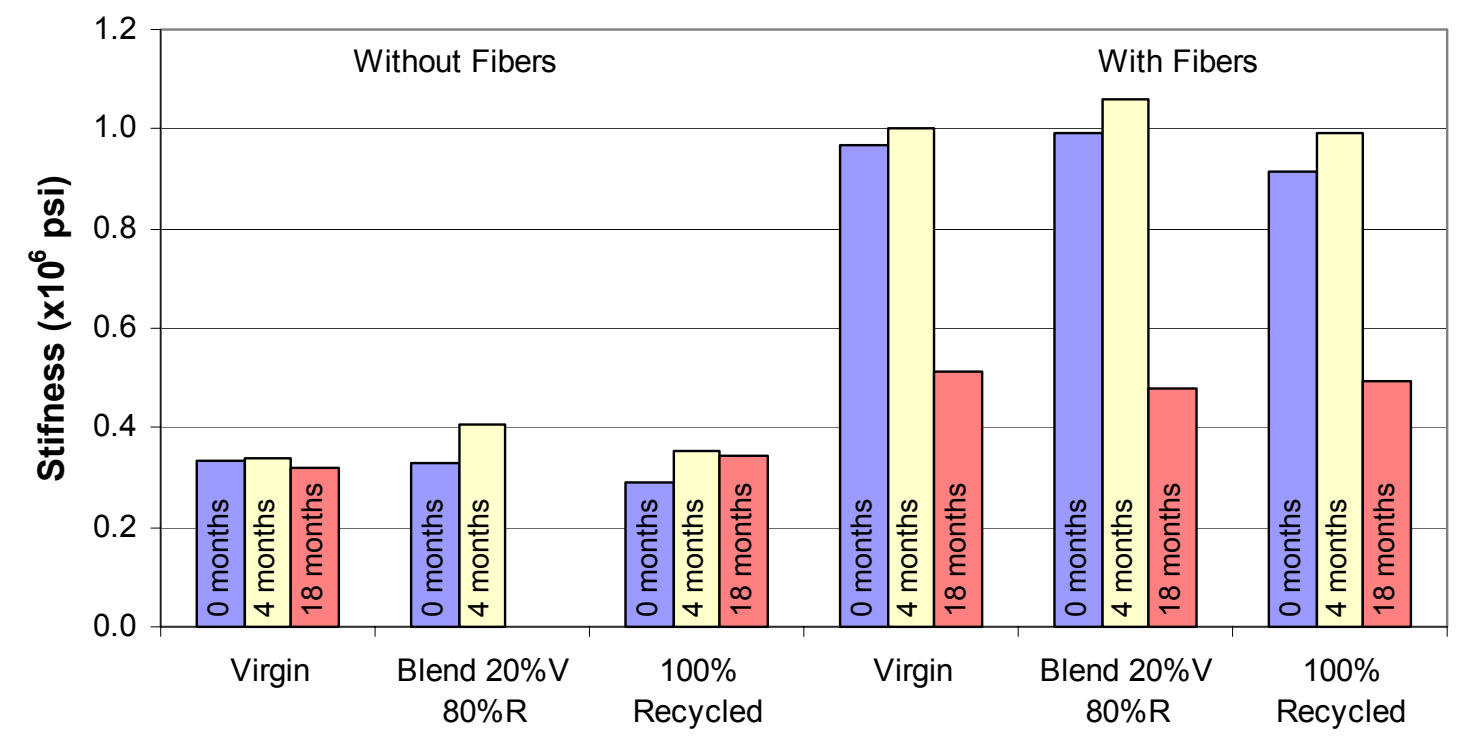

Note: Some intermittent results betw een 0 and 18 months may not be present due to no testing during that period

Figure 5.12 Compressive Stiffness in Aged PC Specimens

\subsubsection{Analysis and Discussion on PC}

Tables 5.11 and F.11 show the average reduction in compressive strength for PC specimens.

\section{Compressive Strength Variation in PC at the End of the Aging Period:}

- Reduction in compressive strength under conditioning varied from $0.1 \%$ to $2.9 \%$ for specimens without fibers and $33.7 \%$ to $41.2 \%$ for specimens with fibers.

- Average reduction in compressive strength after 18 months of conditioning for specimens without fibers was $1.3 \%$ as compared to $37.7 \%$ in specimens with fibers.

\section{Compressive Strength Variation in ABS During the Aging Period:}

- Maximum reductions were noted to be between $1.8 \%$ and $5.6 \%$ for specimens without fibers and $39.0 \%$ to $44.7 \%$ for specimens with fibers. 
- Specimens without fibers showed maximum gains of $4.3 \%$ to $10.1 \%$ whereas no gain was observed for specimens with fibers.

- Average maximum reduction during the aging period was $3.8 \%$ for specimens without fibers as compared to $41.7 \%$ for specimens with fibers.

Tables 5.12, F.12 show average reduction and average change in compressive stiffness for PC samples.

\section{Compressive Stiffness Variation in PC at the End of the Aging Period:}

- No reduction in compressive stiffness under conditioned state was observed for specimens without fibers or specimens with fibers.

- Compressive stiffness gains of $52.4 \%$ to $72.0 \%$ were observed for specimens without fibers and $10.9 \%$ to $24.6 \%$ for specimens with fibers.

\section{Compressive Stiffness Variation in PC During the Aging Period:}

No reductions in bending stiffness were observed. Stiffness gain ranging from $52.4 \%$ to $72.0 \%$ was noted for specimens without fibers as compared to $11.9 \%$ to $24.6 \%$ for specimens with fibers.

Gains observed in some of the specimens may be possibly due to secondary curing effects and improvement in force transfer across the resin/fiber interface.

It should be noted that the compressive stiffness was not measured after 10 months of conditioning and tests may have to be conducted to obtain further results. 


\subsection{Impact Test}

\subsubsection{Results on ABS}

Tables 5.13 and 5.14 show impact strength results for aged ABS specimens. Impact strength is provided for each type of specimen and aging period. Figure 5.13 shows impact strength variation for each of the specimen types under aging.

Table 5.13 Impact Strength Variations in ABS Specimens

\begin{tabular}{|c|c|c|c|c|c|c|}
\hline \multirow{3}{*}{\begin{tabular}{|} 
Specimen \\
Type
\end{tabular}} & \multicolumn{5}{|c|}{ Impact Strength $\left(\mathbf{f t}-\operatorname{lbs}_{\mathrm{f}}\right)$} & \% Change \\
\hline & \multicolumn{5}{|c|}{ Months of aging } & \multirow{2}{*}{$\begin{array}{c}\text { Maximum reduction in } \\
\text { period }\end{array}$} \\
\hline & $\mathbf{0}$ & 2 & 4 & 10 & 18 & \\
\hline A1 & 3.49 & 3.67 & 3.76 & 3.44 & 3.41 & -2.3 \\
\hline A2 & 1.58 & 1.31 & 1.40 & 1.32 & 1.24 & -21.5 \\
\hline A3 & 2.17 & 1.91 & 1.24 & 0.83 & 0.87 & -61.6 \\
\hline A4 & 0.96 & 0.90 & 0.83 & 0.75 & 0.75 & -21.7 \\
\hline A5 & 2.38 & 2.86 & 2.54 & 1.77 & 1.46 & -38.8 \\
\hline A6 & 1.20 & 1.04 & 1.08 & 1.00 & 0.87 & -27.2 \\
\hline \multicolumn{6}{|c|}{ Average reduction without fibers $(\mathrm{A} 1, \mathrm{~A} 3, \mathrm{~A} 5)$} & -34.2 \\
\hline \multicolumn{6}{|c|}{ Average reduction with fibers $(\mathrm{A} 2, \mathrm{~A} 4, \mathrm{~A} 6)$} & -23.5 \\
\hline
\end{tabular}

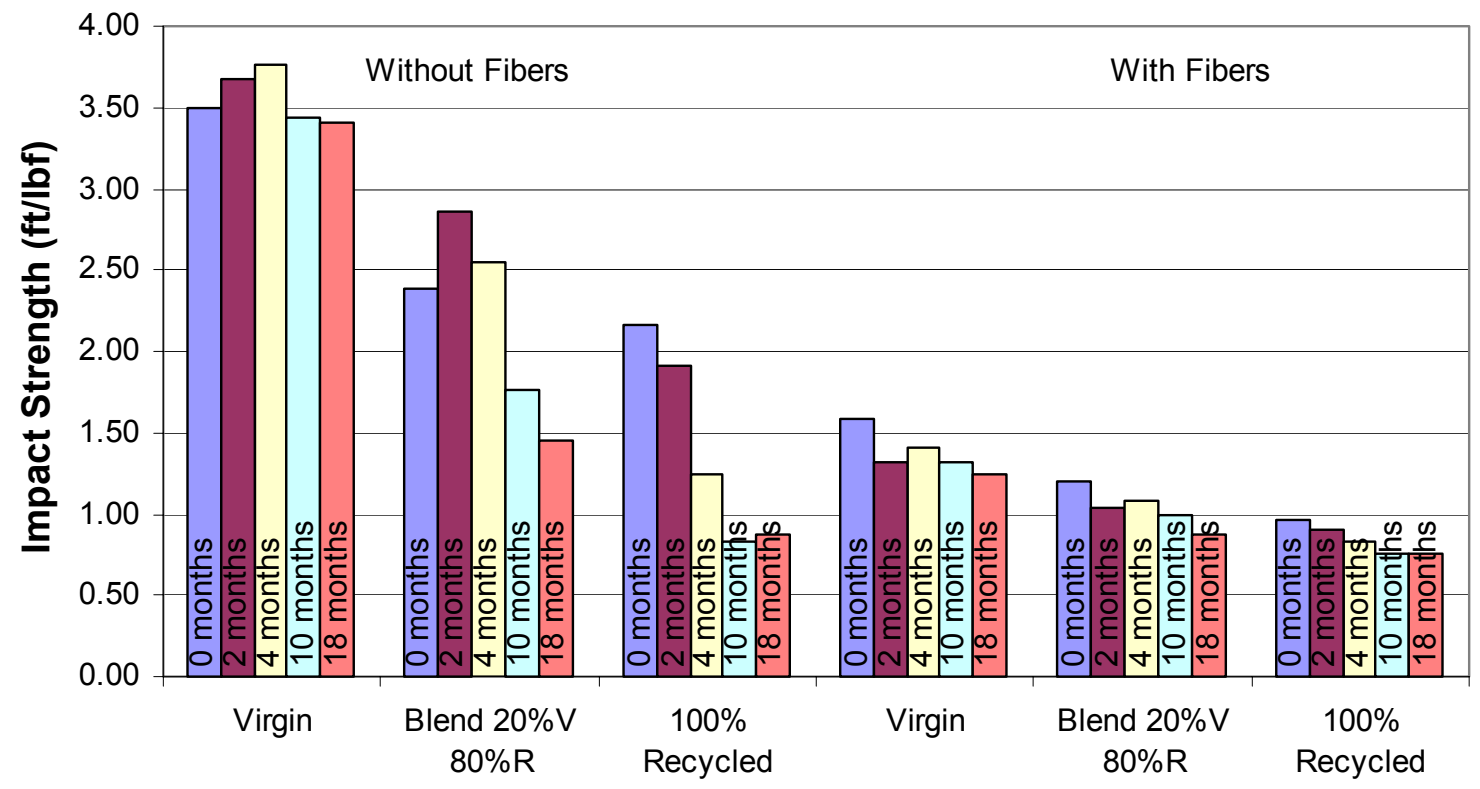

Figure 5.13 Impact Strength in Aged ABS Specimens 


\subsubsection{Analysis and Discussion on ABS}

Tables 5.13 and F.13 show the average reduction in impact strength for ABS specimens.

\section{Impact Strength Variation in ABS at the End of the Aging Period:}

- Reduction in impact strength under conditioning varied from $2.3 \%$ to $59.7 \%$ for specimens without fibers and $21.5 \%$ to $21.7 \%$ for specimens with fibers.

- Average reduction in impact strength after 18 months of conditioning for specimens without fibers was $33.6 \%$ as compared to $23.5 \%$ for specimens with fibers.

\section{Impact Strength Variation in ABS During the Aging Period:}

- Maximum reductions were noted to be ranging from $2.3 \%$ to $61.6 \%$ for specimens without fibers, and $21.5 \%$ to $27.2 \%$ for specimens with fibers.

- Specimens without fibers showed maximum gains of $7.8 \%$ to $20.3 \%$, whereas no gain was observed for specimens with fibers.

- Average maximum reduction during the aging period was $34.2 \%$ for specimens without fibers as compared to $23.5 \%$ for specimens with fibers.

Gains observed in some of the specimens may be possibly due to secondary curing effects and improvement in force transfer across the resin/fiber interface.

\subsubsection{Results on PC}

Tables 5.14 and F.14 show impact strength results for aged PC specimens. Impact strength is provided for each type of specimen and each aging period. Figure 5.14 shows impact strength for aged PC specimens. 
Table 5.14 Impact Strength Variations in Aged PC Specimens

\begin{tabular}{|c|c|c|c|c|c|c|}
\hline \multirow{3}{*}{$\begin{array}{c}\text { Specimen } \\
\text { Type }\end{array}$} & \multicolumn{5}{|c|}{ Impact Strength $\left(\mathbf{f t}-\mathrm{lbs}_{\mathrm{f}}\right)$} & \% Change \\
\hline & \multicolumn{5}{|c|}{ Months of aging } & \multirow{2}{*}{$\begin{array}{c}\text { Maximum reduction in } \\
\text { period }\end{array}$} \\
\hline & $\mathbf{0}$ & 2 & 4 & 10 & 18 & \\
\hline P1 & 14.15 & - & 14.67 & 14.59 & 15.56 & No reduction \\
\hline P2 & 3.01 & - & 1.48 & 1.40 & 1.48 & -53.4 \\
\hline P3 & 13.53 & - & 13.77 & 15.65 & 14.99 & No reduction \\
\hline P4 & 1.81 & - & 0.91 & 0.91 & 0.79 & -56.2 \\
\hline P5 & 14.80 & - & 14.58 & 14.91 & 15.65 & -1.5 \\
\hline P6 & 2.67 & - & 1.24 & 1.32 & 1.20 & -55.1 \\
\hline \multicolumn{6}{|c|}{ Average reduction without fibers (A1,A3,A5) } & -1.5 \\
\hline \multicolumn{6}{|c|}{ Average reduction with fibers $(\mathrm{A} 2, \mathrm{~A} 4, \mathrm{~A} 6)$} & -54.9 \\
\hline
\end{tabular}

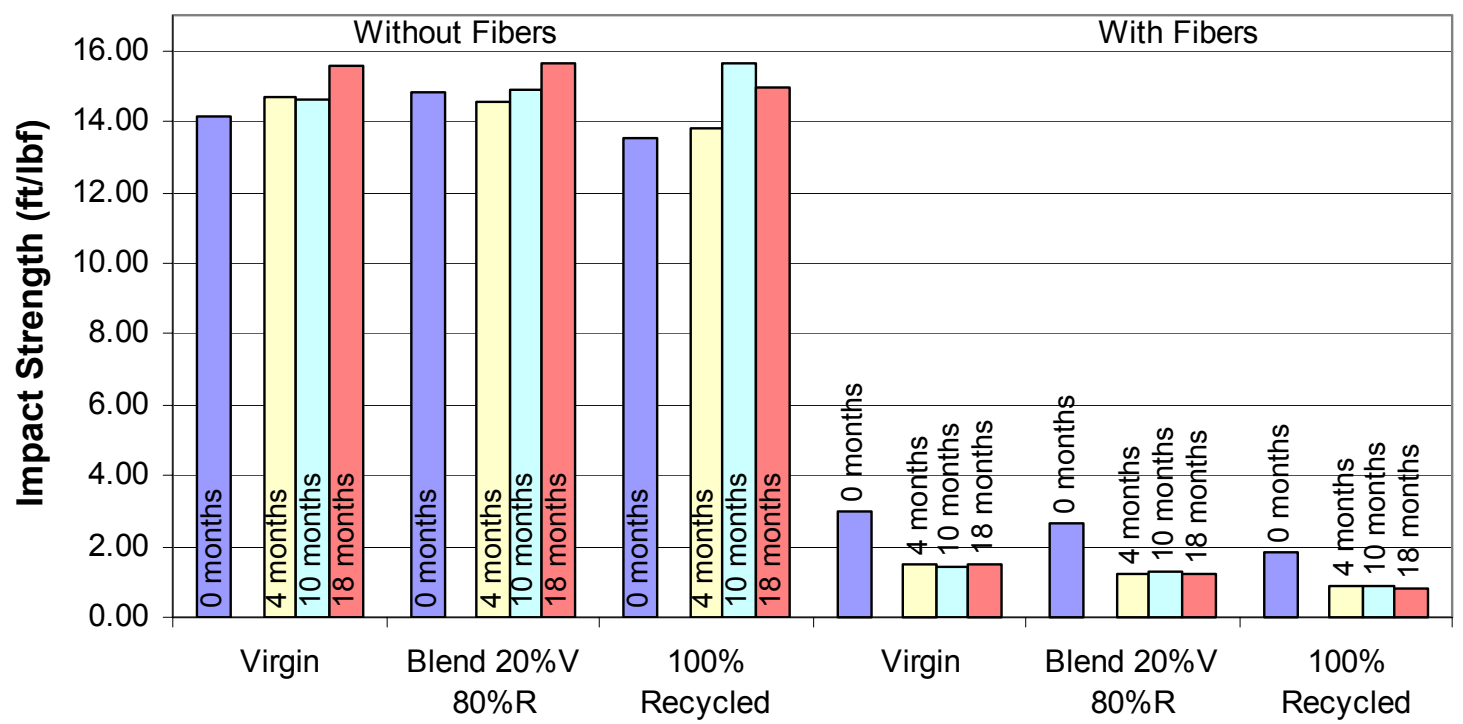

Note: Some intermittent results betw een 0 and 18 months may not be present due to no testing during that period

Figure 5.14 Impact Strength in Aged PC Specimens

\subsubsection{Analysis and Discussion on PC}

Tables 5.14 and F.14 show the average reduction in impact strength for PC samples. 


\section{Impact Strength Variation in PC at the End of the Aging Period:}

- No reduction in impact strength under conditioning was observed for specimens without fibers and a reduction of $50.7 \%$ to $56.2 \%$ was noted for specimens with fibers.

- Average reduction in impact strength was 54.0\% after 18 months of conditioning for samples with fibers.

\section{Impact Strength Variation in PC During the Aging Period:}

- Maximum reductions were noted to be ranging from $1.5 \%$ to zero for specimens without fibers and $53.4 \%$ to $56.2 \%$ for specimens with fibers.

- Specimens without fibers showed maximum gains of $5.7 \%$ to $15.6 \%$ whereas no gain was observed for specimens with fibers.

- Maximum average reduction after aging was $1.5 \%$ for specimens without fibers as compared to $54.9 \%$ for specimens with fibers.

Gains observed in some of the specimens may be possibly due to secondary curing effects and improvement in force transfer across the resin/fiber interface.

\subsection{Hardness Test}

\subsubsection{Results on ABS}

Tables 5.15 and F.15 show hardness test data for ABS specimens. Hardness index is provided for each type of specimen and each aging period. Figure 5.15 shows hardness index for each of the specimen types and aging periods, respectively. 
Table 5.15 Hardness Index Variations in Aged ABS Specimens

\begin{tabular}{|c|c|c|c|c|c|c|}
\hline \multirow{2}{*}{$\begin{array}{c}\text { Specimen } \\
\text { Type }\end{array}$} & \multicolumn{5}{|c|}{ Hardness Index } & \% Change \\
\cline { 2 - 6 } & \multicolumn{5}{|c|}{ Months of aging } & $\begin{array}{c}\text { Maximum reduction in } \\
\text { period }\end{array}$ \\
\cline { 2 - 6 } & $\mathbf{0}$ & $\mathbf{2}$ & $\mathbf{4}$ & $\mathbf{1 0}$ & $\mathbf{1 8}$ & No reduction. \\
\hline $\mathrm{A} 1$ & 10.90 & 12.44 & 11.70 & 12.10 & 12.00 & -3.5 \\
\hline $\mathrm{A} 2$ & 11.30 & 12.80 & 11.50 & 11.00 & 10.90 & -0.9 \\
\hline A3 & 11.10 & 12.58 & 11.40 & 11.10 & 11.00 & -5.4 \\
\hline A4 & 11.20 & 11.76 & 11.30 & 10.80 & 10.60 & -3.6 \\
\hline A5 & 11.20 & 12.44 & 10.80 & 11.10 & 10.90 & -3.6 \\
\hline A6 & 11.20 & 12.16 & 11.00 & 10.90 & 10.80 & -2.2 \\
\hline Average reduction without fibers (A1,A3,A5) & -4.2 \\
\hline
\end{tabular}

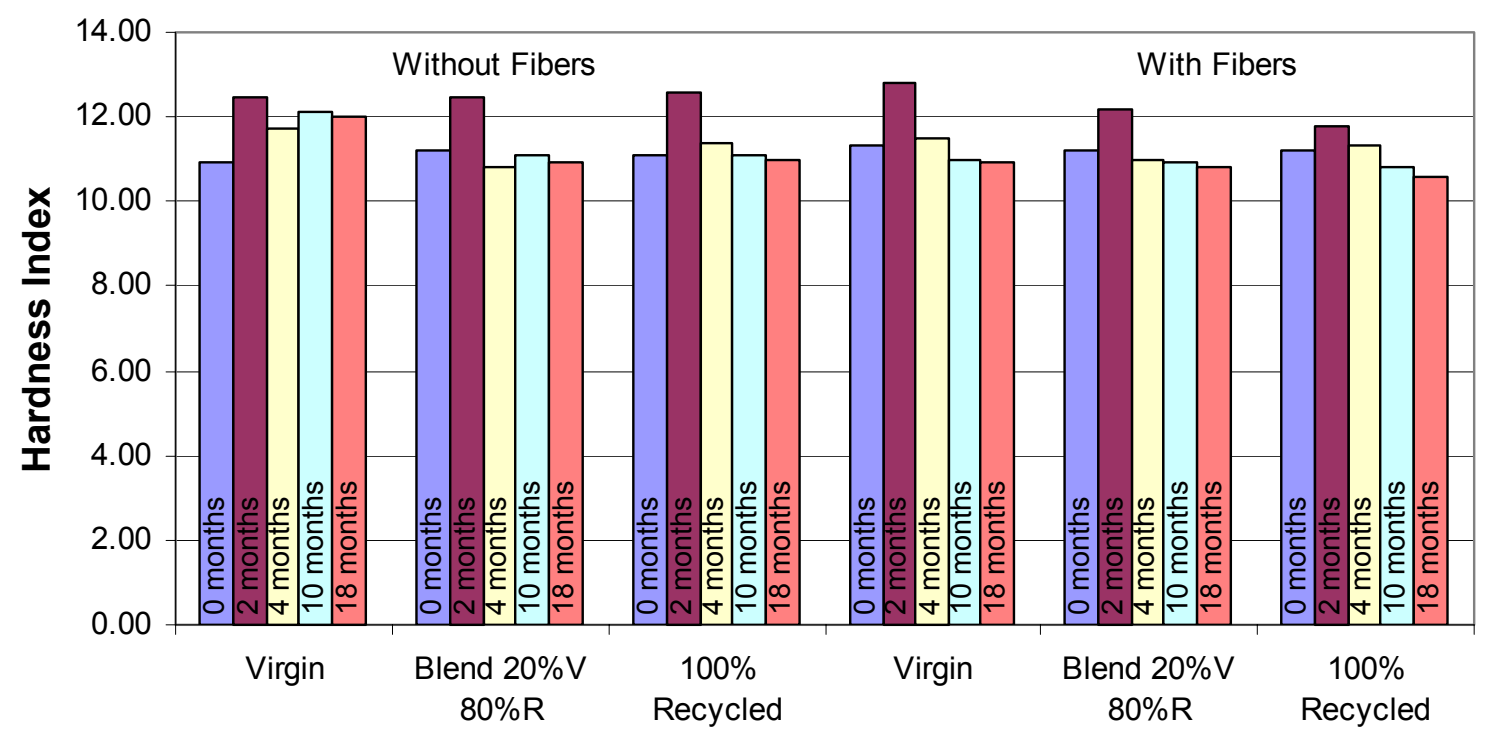

Figure 5.15 Hardness Index in Aged ABS Specimens

\subsubsection{Analysis and Discussion on ABS}

Tables 5.15 and F.15 show average reduction in hardness index for ABS samples.

\section{Hardness Index Variation in ABS at the End of the Aging Period:}

- Reduction in hardness index under conditioning varied from $0.9 \%$ to $2.7 \%$ for specimens without fibers and $3.5 \%$ to $5.4 \%$ for specimens with fibers. 
- Average reduction in impact strength after 18 months of conditioning for specimens without fibers was $1.8 \%$ as compared to $4.2 \%$ for specimens with fibers.

\section{Hardness Index Variation in ABS During the Aging Period:}

- Maximum reductions ranged from $0.9 \%$ to $3.6 \%$ for specimens without fibers and $3.5 \%$ to $5.4 \%$ for specimens with fibers.

- Specimens without fibers showed maximum gains of $11.1 \%$ to $14.1 \%$, whereas gains of $5.0 \%$ to $13.3 \%$ were observed for specimens with fibers.

- Maximum average reduction during the aging period was $2.2 \%$ for specimens without fibers as compared to $4.2 \%$ for specimens with fibers.

Gains observed in some of the specimens may be possibly due to secondary curing effects and improvement in force transfer across the resin/fiber interface.

\subsubsection{Results on PC}

Tables 5.16 and F.16 show hardness results for aged PC specimens. Hardness index is provided for each type of specimen and each aging period. Figure 5.16 shows hardness index for each of the specimen types and aging periods, respectively.

Table 5.16 Hardness Index Variations in Aged PC Specimens

\begin{tabular}{|c|c|c|c|c|c|c|}
\hline \multirow{2}{*}{$\begin{array}{c}\text { Specimen } \\
\text { Type }\end{array}$} & \multicolumn{5}{|c|}{ Hardness Index } & \% Change \\
\cline { 2 - 7 } & \multicolumn{7}{|c|}{ Months of aging } & $\begin{array}{c}\text { Maximum reduction in } \\
\text { period }\end{array}$ \\
\cline { 2 - 7 } & $\mathbf{0}$ & $\mathbf{2}$ & $\mathbf{4}$ & $\mathbf{1 0}$ & $\mathbf{1 8}$ & No reduction \\
\hline P1 & 10.60 & 12.00 & 11.30 & 11.10 & 11.00 & No reduction \\
\hline P2 & 10.70 & 11.10 & 11.20 & 11.30 & 11.30 & No reduction \\
\hline P3 & 10.60 & 11.50 & 10.80 & 11.10 & 11.00 & No reduction \\
\hline P4 & 10.70 & 11.44 & 10.80 & 10.90 & 10.80 & No reduction \\
\hline P5 & 10.70 & 10.74 & 11.20 & 10.90 & 10.90 & No reduction \\
\hline P6 & 10.70 & 11.38 & 11.40 & 10.90 & 10.80 & No reduction \\
\hline Average reduction without fibers (A1,A3,A5) \\
\hline Average reduction with fibers (A2,A4,A6)
\end{tabular}




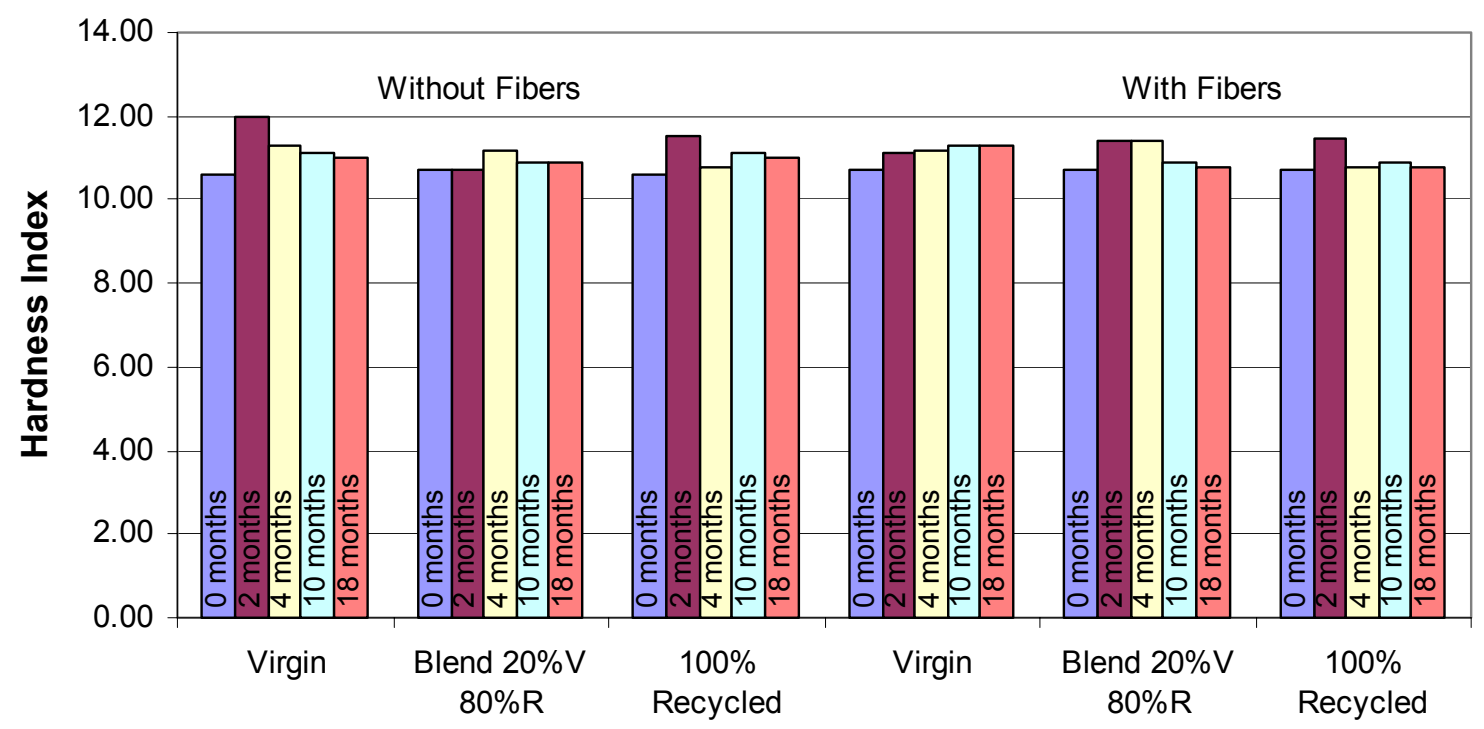

Figure 5.16 Hardness Index in Aged PC Specimens

\subsubsection{Analysis and Discussion on PC}

Tables 5.16 and F.16 show the average reduction in hardness index for PC specimens.

\section{Hardness Index Variation in PC at the End of the Aging Period:}

- Hardness index under conditioning did not vary either for specimens without fibers or for specimens with fibers.

- Gain in hardness index for specimens without fibers varied from $1.9 \%$ to $3.8 \%$ and from $0.9 \%$ to $5.6 \%$ for specimens with fibers.

\section{Hardness Index Variation in PC During the Aging Period:}

No reductions in hardness index were observed for any types of specimens. Specimens without fibers showed maximum gains of $4.7 \%$ to $13.2 \%$, whereas gains of $5.6 \%$ to $6.9 \%$ were observed for specimens with fibers. 
Gains observed in some of the specimens may be possibly due to secondary curing effects and improvement in force transfer across the resin/fiber interface.

\section{$5.7 \quad$ Creep Test}

\subsubsection{Results on ABS}

Tables 5.17 and 5.18 show creep results for ABS specimens tested at sustained load levels of $20 \%$ and $50 \%$ of ultimate (failure) load. Creep coefficient (creep strain / initial strain) is provided for each type of specimen for the testing period. Figures 5.17 and 5.18 show creep coefficient for each of the specimen types during the testing period for sustained loads of $20 \%$ and $50 \%$ of ultimate load, respectively.

Table 5.17 Creep Test Results for 20\% Sustained Load on ABS Specimens

\begin{tabular}{|c|c|c|}
\hline Type of Specimen & Days & $\begin{array}{c}\text { Creep } \\
\text { Coefficient }\end{array}$ \\
\hline A1 - Virgin without fibers & 896 & 1.62 \\
\hline A2 - Virgin with 25\%(wt.\%) chopped fibers & 896 & 0.82 \\
\hline A3 $-100 \%$ recycled without fibers & 924 & 1.73 \\
\hline A4 $-100 \%$ recycled with $25 \%(w t . \%)$ chopped fibers & 896 & 0.72 \\
\hline A5 - Recycled (20\%) / virgin $(80 \%)$ blend without fibers & 924 & 2.07 \\
\hline $\begin{array}{l}\text { A6 - Recycled (20\%) / virgin }(80 \%) \text { blend with } 25 \%(\text { wt. } \%) \\
\text { chopped fibers }\end{array}$ & 896 & 0.95 \\
\hline
\end{tabular}




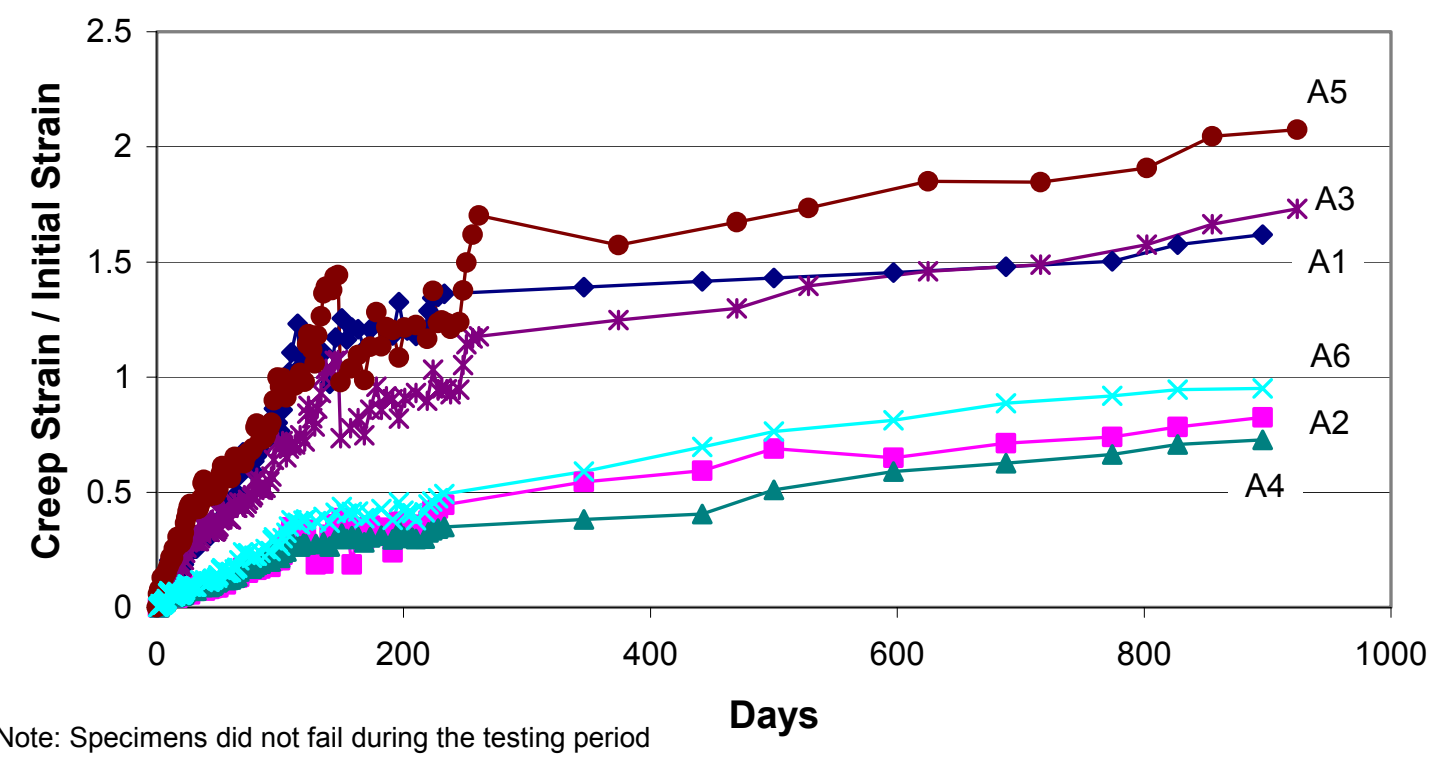

Figure 5.17 Creep Test on ABS Specimens for 20\% Sustained Load

Table 5.18 Creep Test Results for 50\% Sustained Load on ABS Specimens

\begin{tabular}{|l|c|c|}
\hline \multicolumn{1}{|c|}{ Type of Specimen } & Days & $\begin{array}{c}\text { Creep } \\
\text { Coefficient }\end{array}$ \\
\hline A1 - Virgin without fibers & 44 (Failed) & 0.99 (Failed) \\
\hline A2 - Virgin with 25\%(wt.\%) chopped fibers & 944 & 1.59 \\
\hline A3 - 100\% recycled without fibers & 39 (Failed) & 0.94 (Failed) \\
\hline A4 - 100\% recycled with 25\%(wt.\%) chopped fibers & 944 & 2.18 \\
\hline A5 - Recycled (20\%) / virgin (80\%) blend without fibers & 39 (Failed) & 1.18 (Failed) \\
\hline A6 - Recycled (20\%) / virgin (80\%) blend with & 944 & 1.70 \\
25\%(wt.\%) chopped fibers & & \\
\hline
\end{tabular}




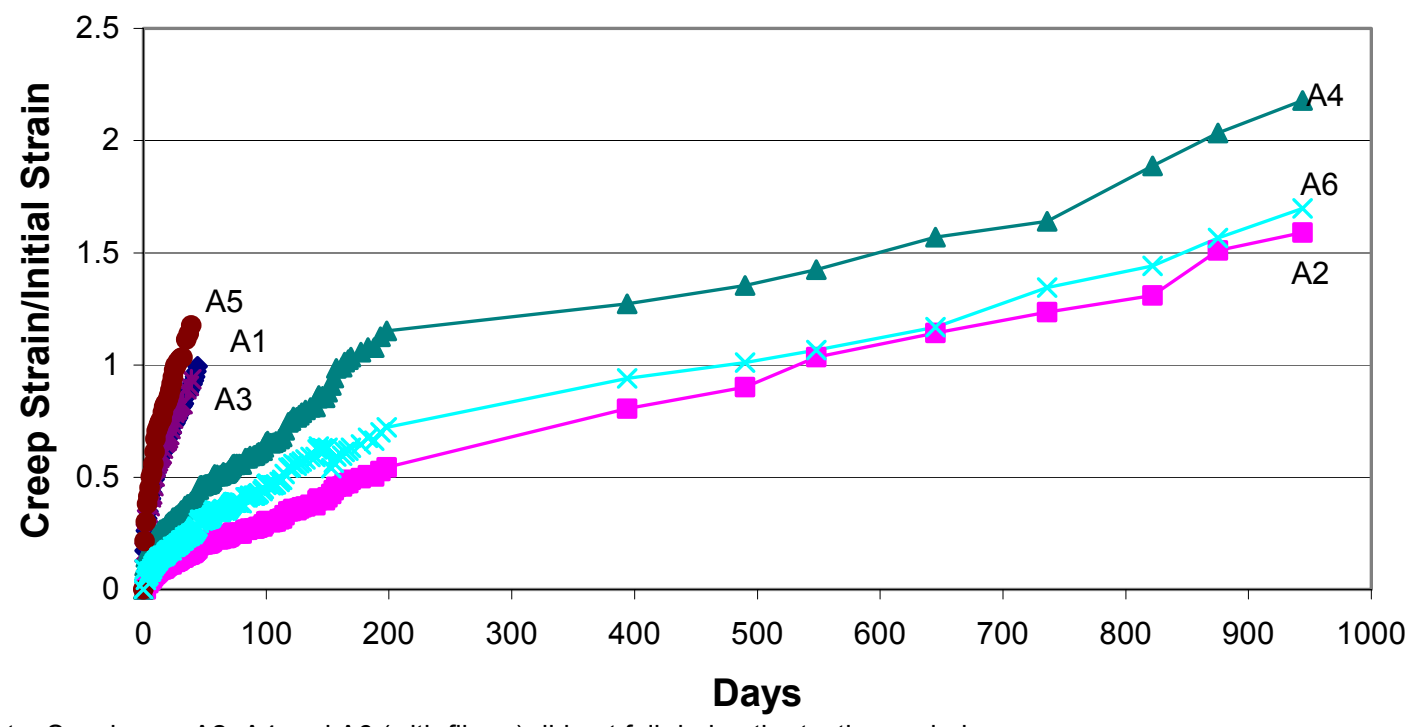

Note: Specimens A2, A4 and A6 (with fibers) did not fail during the testing period

Figure 5.18 Creep Test on ABS Specimens for 50\% Sustained Load

\subsubsection{Analysis on ABS}

For ABS specimens tested under $20 \%$ sustained load, creep coefficient varied from 1.62 to 2.07 for specimens without fibers on 924th day and 0.72 to 0.95 for specimens with fibers on 896th day of testing. Average creep coefficient after 924 days of testing for samples without fibers was 1.81 as compared to 0.83 for samples with fibers after 896 days.

ABS specimens without fibers tested at 50\% sustained load failed with a maximum creep coefficient of 0.94 and 1.18 on 39 th day for $100 \%$ recycled and blend specimens, respectively, and 0.99 on $44^{\text {th }}$ day for virgin ABS specimens.

Specimens with fibers tested at 50\% sustained load, showed a creep coefficient between 1.59 and 2.18 on the $944^{\text {th }}$ day. Average creep coefficient of 1.82 was observed for ABS specimens with fibers. 
For ABS specimens with fibers, average creep coefficient after 924 days increased from 0.83 when tested under $20 \%$ sustained load to 1.82 for $50 \%$ sustained load.

ABS specimens without fabrics failed within a short period (39 to 44 days) when tested under $50 \%$ sustained load. However, ABS specimens without fabrics tested under $20 \%$ sustained load showed an average creep coefficient of 1.81 after 924 days without fail.

During the testing period, higher creep strains were observed for specimens without fibers, indicating that the addition of fibers decreases the long-term deformation of ABS specimens under sustained loads.

\subsubsection{Results on PC}

Tables 5.19 and 5.20 show creep results for PC specimens tested at sustained load levels of $20 \%$ and $50 \%$ of ultimate load. Creep coefficient (creep strain / initial strain) is provided for each type of specimen for the testing period. Figures 5.19 and 5.20 show creep coefficient for each of the specimen types during the testing period for sustained loads of $20 \%$ and $50 \%$ of ultimate load, respectively.

Table 5.19 Creep Test Results for 20\% Sustained Load on PC Specimens at 809

Days

\begin{tabular}{|l|c|}
\hline \multicolumn{1}{|c|}{ Type of Specimen } & Creep Coefficient \\
\hline P1 - Virgin without fibers & 0.50 \\
\hline P2 - Virgin with 25\%(wt.\%) chopped fibers & 0.35 \\
\hline P3 - 100\% recycled without fibers & 0.50 \\
\hline P4 - 100\% recycled with 25\%(wt.\%) chopped fibers & 0.22 (failed on $23^{\text {rd }}$ day) \\
\hline P5 - Recycled (20\%) / virgin (80\%) blend without fibers & 0.51 \\
\hline $\begin{array}{c}\text { P6 - Recycled (20\%)/ virgin (80\%) blend with 25\%(wt.\%) } \\
\text { chopped fibers }\end{array}$ & 0.37 \\
\hline
\end{tabular}




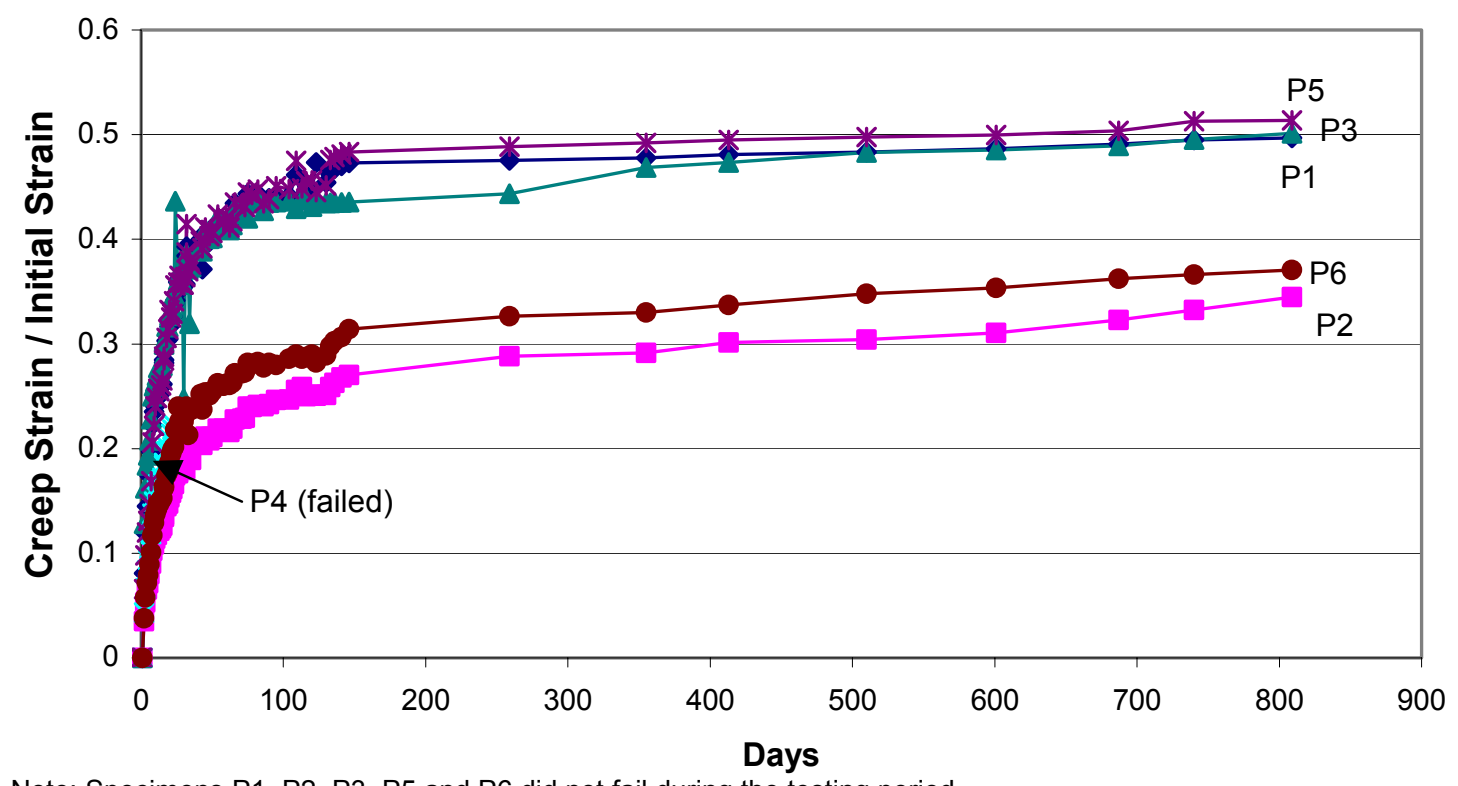

Note: Specimens P1, P2, P3, P5 and P6 did not fail during the testing period

Figure 5.19 Creep Test on PC Specimens for 20\% Sustained Load

Table 5.20 Creep Test Results for 50\% Sustained Load on PC Specimens at 809

Days

\begin{tabular}{|l|c|}
\hline \multicolumn{1}{|c|}{ Type of Specimen } & Creep Coefficient \\
\hline P1 - Virgin without fibers & 0.77 \\
\hline P2 - Virgin with 25\%(wt.\%) chopped fibers & 0.47 \\
\hline P3 - 100\% recycled without fibers & 0.86 \\
\hline P4 - 100\% recycled with 25\%(wt.\%) chopped fibers & 0.44 \\
\hline P5 - Recycled (20\%) / virgin (80\%) blend without fibers & 0.80 \\
\hline $\begin{array}{l}\text { P6 - Recycled (20\%) / virgin (80\%) blend with 25\%(wt.\%) } \\
\text { chopped fibers }\end{array}$ & 0.40 \\
\hline
\end{tabular}




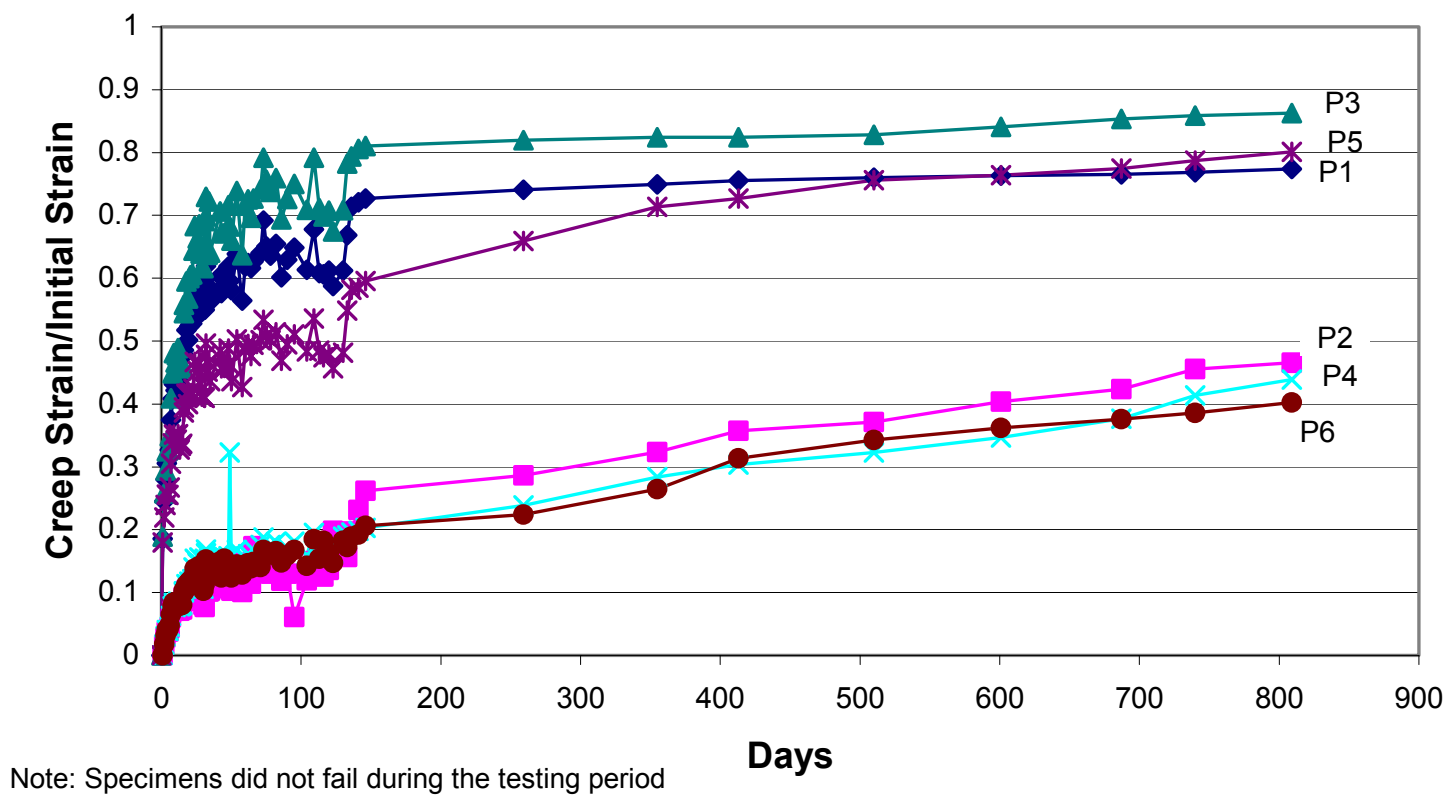

Figure 5.20 Creep Test on PC Specimens for 50\% Sustained Load

\subsubsection{Analysis on PC}

Specimen P4 (100\% recycled with chopped fibers) tested under $20 \%$ sustained load failed with a maximum creep coefficient of 0.22 on 23 rd day.

On the $809^{\text {th }}$ day of testing, specimens P2 and P6 (remaining specimens with fibers) tested at $20 \%$ sustained load showed a creep coefficient between 0.35 and 0.37 as compared to 0.50 to 0.51 for specimens without fibers at $20 \%$ sustained load. Average creep coefficient of 0.36 was observed for PC specimens with fibers and 0.50 for specimens without fibers.

For PC specimens tested under $50 \%$ sustained load, creep coefficient on $809^{\text {th }}$ day of testing varied from 0.77 to 0.86 for specimens without fibers and 0.40 to 0.47 for specimens with fibers. Average creep coefficient after 809 days of testing for samples without fibers was 0.81 as compared to 0.44 for samples with fibers. 
For PC specimens with fibers, average creep coefficient after 809 days increased from 0.36 when tested under $20 \%$ sustained load to 0.44 for $50 \%$ sustained load.

For PC specimens without fibers, average creep coefficient after 809 days increased from 0.50 when tested under $20 \%$ sustained load to 0.81 for $50 \%$ sustained load.

Similar to ABS specimens, higher creep strains were observed for PC specimens without fibers, indicating that the addition of fibers decreases the long-term deformation under sustained loads.

At $20 \%$ of sustained load, PC specimens without fibers showed one-third the creep coefficient of ABS specimens (0.50 vs. 1.81). With fiber addition, creep coefficient in PC was near one half the creep coefficient in ABS specimens at $20 \%$ load, i.e., ( 0.36 vs. 0.83$)$.

Sustained load level on ABS specimens is suggested to be limited to $20 \%$. At $50 \%$ of sustained load, PC specimens showed better capacity to carry sustained load than ABS specimens, either with or without fibers.

For reinforced concrete, creep coefficient at a given time with respect to initial loading time can be computed as (Nilson, 1986):

$$
C_{c t}=\frac{t^{0.60}}{10+t^{0.60}} C_{c u}
$$

Where,

Cct $=$ Creep coefficient after $t$ days of loading

$\mathrm{t}=$ time in days after loading

$\mathrm{Ccu}=$ Ultimate creep coefficient (2.9 for a $4,000 \mathrm{psi}$ reinforced concrete) 
Calculated creep coefficients for reinforced concrete at the $809^{\text {th }}, 896^{\text {th }}$, and $924^{\text {th }}$ day after loading are 2.46, 2.48, and 2.49, respectively. Compared to ABS and PC specimens tested on this research, concrete creep coefficient is at least $13 \%$ higher than that observed for ABS and PC specimens without fibers and more than 1.5 times higher than that of ABS and PC specimens with fibers. 


\section{CHAPTER 6}

\section{TEST RESULTS, ANALYSIS AND DISCUSSIONS ON GUARDRAIL POST, RAIL AND OFFSET BLOCK}

\subsection{Introduction}

As described in chapter 4, several static tests such as tension, compression and bending were performed on coupon and prototype specimens made of recycled polymer and discarded rubber tires to determine their suitability for highway guardrail system. In this chapter, test results on different types of specimens including shapes are presented, discussed and compared.

\subsection{Recycled Polypropylene Channel Section}

\subsubsection{Test Results on Recycled Polypropylene Channel Section}

Compressive and tensile gage locations and stress-strain variations for the channel section are shown in Figures 6.1 and 6.2, respectively. The response shown in Figure 6.2 is for a 3 point bending test on the recycled polypropylene channel section. Maximum experimental stress and stiffness values of the section are shown in Table 6.1. Punching failure occurred on the top member. Fiber volume fraction of the section was found to be $43 \%$ based on ignition test. Stiffnesses were computed from the slope of strain versus stress plots for tensile and compressive stress (Figure 6.2) using linear curve fit. Computation of stresses was made as follows:

$$
\sigma=\frac{M c}{I}
$$

Where,

$\sigma=$ Compressive or tensile bending stress

$\mathrm{M}=$ Moment at midspan for 3-point bending 


$$
\begin{aligned}
M= & \frac{P L}{4} \\
& \begin{array}{l}
\mathrm{P}=\text { Applied load } \\
\mathrm{L}=\text { Span length }
\end{array}
\end{aligned}
$$

$c=$ Distance from section centroid to point of stress calculation (Figure 6.1).

$\mathrm{I}=$ Moment of inertia about centroid of section $=0.0841 \mathrm{in}^{4}$

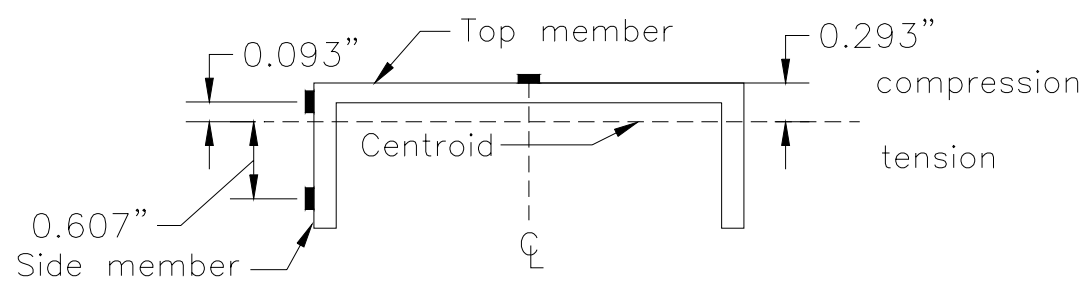

Figure 6.1 Distances from Centroid of Channel Section to Locations of Strain Gages

Table 6.1 Maximum Tensile and Compressive Stresses and Stiffnesses of Polypropylene Channel Section Under Three Point Bending Test

\begin{tabular}{|c|c|c|c|c|}
\hline Location & $\begin{array}{c}\text { Type of } \\
\text { stress }\end{array}$ & $\begin{array}{c}\text { Stiffness, E } \\
\text { (psi) }\end{array}$ & $\begin{array}{c}\text { Maximum } \\
\text { Stress } \\
\text { (psi) }\end{array}$ & Failure mode \\
\hline $\begin{array}{c}\text { Top @ midspan and } \\
\text { center of channel width }\end{array}$ & Compression & $2.45 \times 10^{6}$ & 6,686 & $\begin{array}{c}\text { Punching on } \\
\text { compression side }\end{array}$ \\
\hline $\begin{array}{c}\text { Bottom @ midspan on } \\
\text { bottom-most fiber on the } \\
\text { side of the channel }\end{array}$ & Tension & $2.55 \times 10^{6}$ & 13,831 & \\
\hline
\end{tabular}




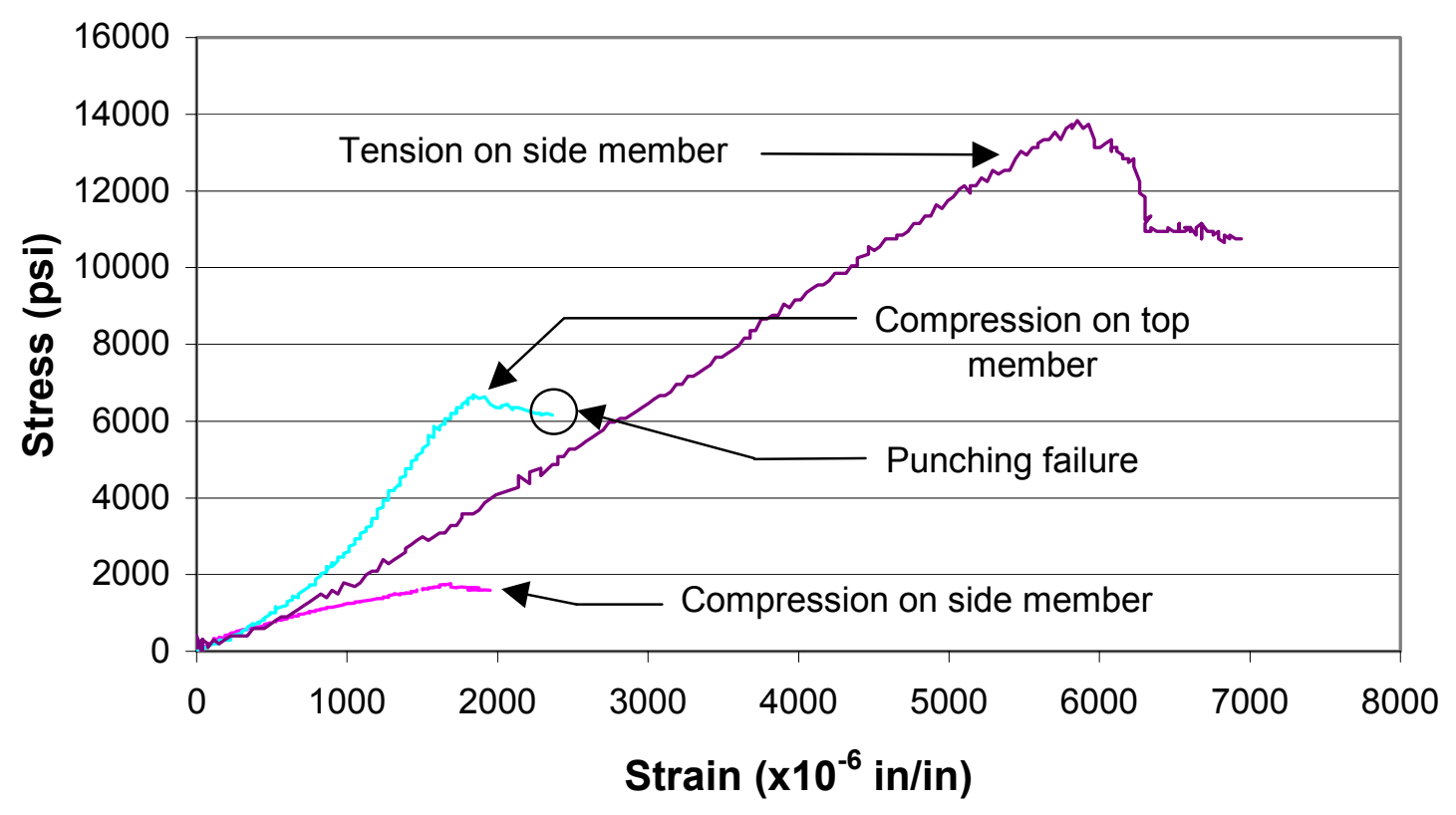

Figure 6.2 Compressive and Tensile Stresses in Recycled Polypropylene Channel Section Under Three Point Bending Test

\subsubsection{Analysis and Discussion of Test Results on a Recycled Polypropylene Channel Section}

Figure 6.2 shows the stress versus strain for a channel section under bending loads. Tensile stresses on the bottom were found to be higher than the top compressive stresses. This is due to the fact that the fiber area in tension zone is less than the fiber area in the compression zone, which consists of both horizontal portion and vertical legs (Figure 6.1). Failure occurred on the top of the channel, when compressive stress reached 6,686 psi, and tensile stress was 13,831 psi. Considering that the entire section was uniformly reinforced, it was evident from the failure type that the section with a thickness of 0.17 " was punched through under the loading point. 


\subsection{Recycled Polypropylene Trapezoidal Section}

\subsubsection{Test Results on Recycled Polypropylene Trapezoidal Section}

Trapezoidal section with recycled polypropylene is shown in Figure 6.3. This section had $43 \%$ fiber volume fraction as determined by ignition test.

Bending: Compressive and tensile stresses at the locations shown in Figure 6.3 during 3 point bending tests are plotted in Figure 6.4. Bending tests were conducted on the trapezoidal section and tension tests were conducted on the plates cut from trapezoidal section. Maximum stress and stiffness values are shown in Table 6.2. A combination of compression and punching failure was observed on the top member. Stiffnesses were computed from the slope of the linear curve fit of the stress versus strain plots for compressive and tensile stress (Figure 6.4). Moment of inertia of the section (I) was computed and the value is $0.0433 \mathrm{in}^{4}$. Bending stresses were calculated as described for the channel section. Outer fiber distances from neutral axis for stress calculations are given in Figure 6.3.

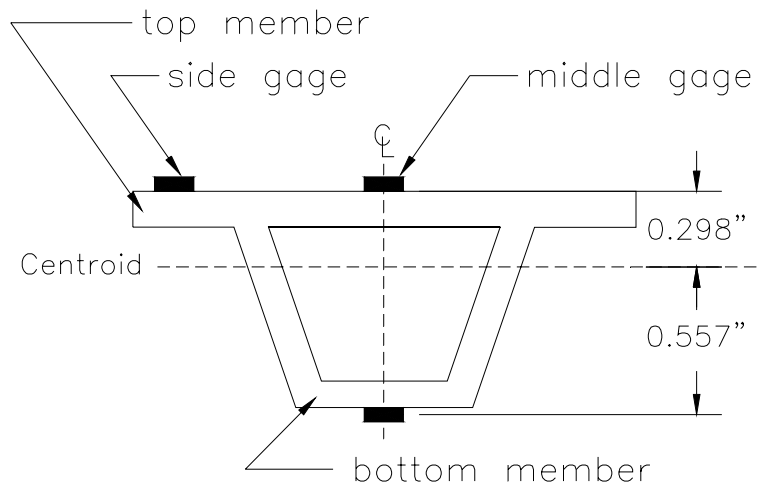

Figure 6.3 Distances from Centroid of Trapezoidal Section to Locations of Strain Gages 
Table 6.2 Maximum Tensile and Compressive Stresses and Stiffnesses of Polypropylene Trapezoidal Section Under Three Point Bending Test

\begin{tabular}{|c|c|c|c|c|}
\hline Location & Type of stress & $\begin{array}{c}\text { Stiffness, E } \\
(\mathbf{p s i})\end{array}$ & $\begin{array}{c}\text { Maximum } \\
\text { Stress } \\
(\mathbf{p s i})\end{array}$ & Failure mode \\
\hline $\begin{array}{c}\text { Top member @ center } \\
\text { of the section }\end{array}$ & Compression & $3.04 \times 10^{6}$ & 23,722 & Punching \\
\hline $\begin{array}{c}\text { Bottom member @ } \\
\text { center of the section }\end{array}$ & Tension & $3.16 \times 10^{6}$ & 45,049 & \\
\hline
\end{tabular}

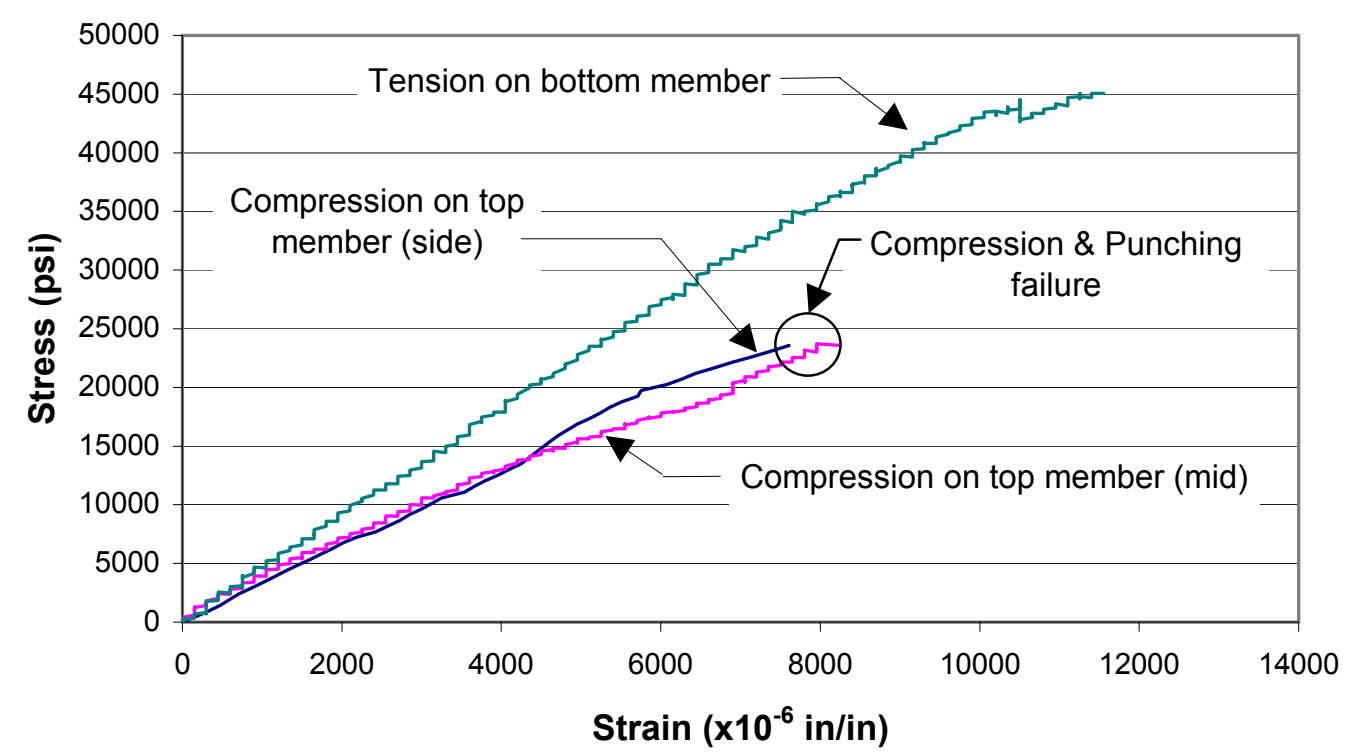

Figure 6.4 Compressive and Tensile Bending Stresses on Recycled Polypropylene Trapezoidal Section Under Three Point Bending Test

Tension tests on plates cut from trapezoidal section: For the tension specimens described in section 4.4.1, tension tests were carried out using three different grip configurations and lengths: 1) no grips, 2) $1 \frac{3 / 4}{4}$ in, and 3) $2 \frac{1}{2}$ in. Results of the tension tests are summarized in Table 6.3. Stiffnesses were computed from the slope of the linear curve fit of the stress vs. strain curves (Figure 6.5). Tensile stresses were computed as follows: 
$\sigma=\frac{P}{A}$

Where

$\sigma=$ Tensile stress

$\mathrm{P}=$ Applied load

$A=$ Area of cross section

Table 6.3 Maximum Tensile Stresses and Stiffnesses on Polypropylene Plates from Trapezoidal Section - Tension Test

\begin{tabular}{|c|c|c|c|}
\hline $\begin{array}{c}\text { Location from } \\
\text { where the plate was } \\
\text { cut }\end{array}$ & Grips & Stiffness, E (psi) & $\begin{array}{c}\text { Maximum Stress } \\
\text { (psi) }\end{array}$ \\
\hline Upper Flange - Side & NO & $5.00 \times 10^{6}$ & 53,240 \\
\hline $\begin{array}{c}\text { Upper Flange }- \\
\text { Center }\end{array}$ & $134^{\prime \prime}$ & $3.30 \times 10^{6}$ & 39,124 \\
\hline Web & $21 / 2^{\prime \prime}$ & $3.83 \times 10^{6}$ & 82,897 \\
\hline Web & NO & $4.40 \times 10^{6}$ & 55,000 \\
\hline Bottom Flange & $13 / 4^{\prime \prime}$ & $3.73 \times 10^{6}$ & 72,811 \\
\hline
\end{tabular}

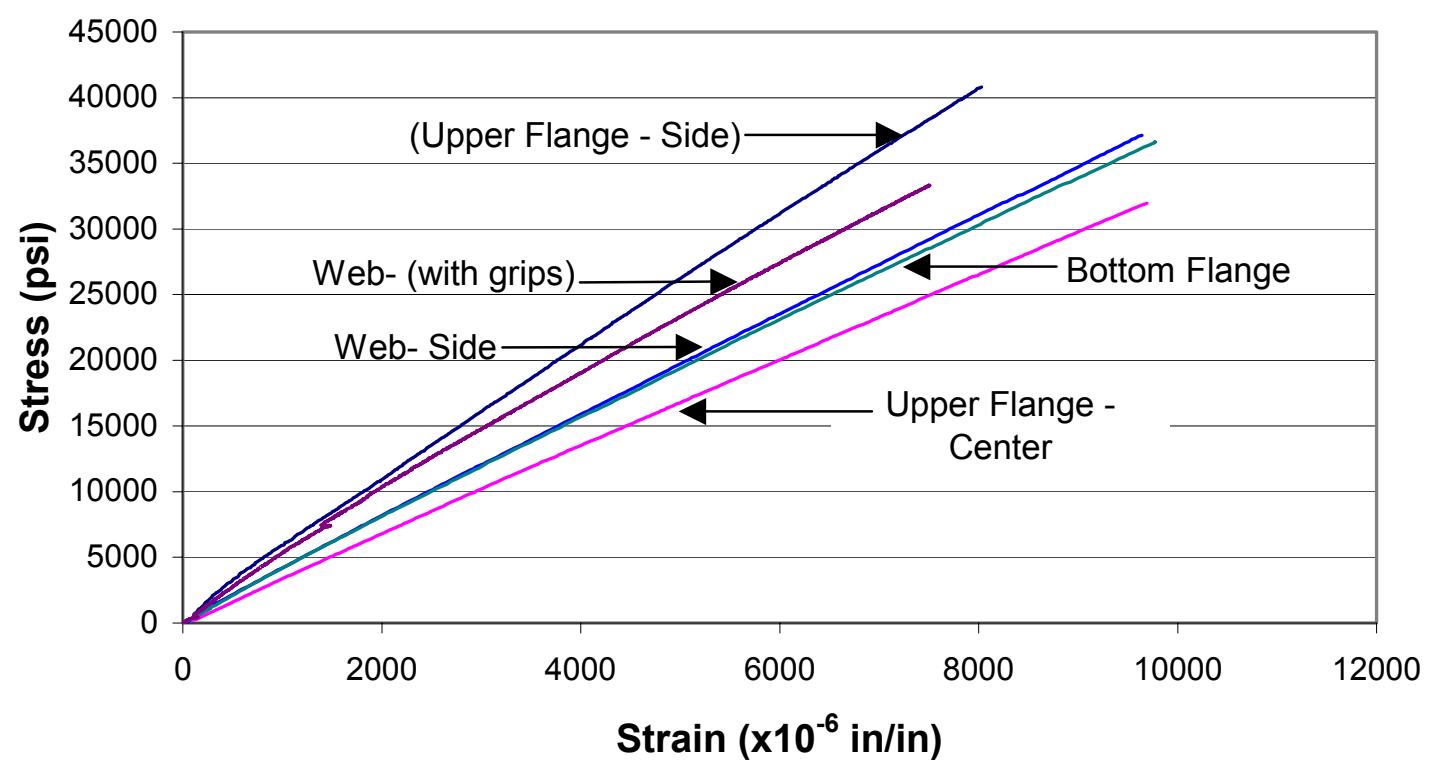

Note: Failure point not shown

Figure 6.5 Linear Portion of Stress vs. Strain for Tension Tests on Recycled Polypropylene Plates from Trapezoidal Section 


\subsubsection{Analysis and Discussion of Test Results on Recycled Polypropylene Trapezoidal Section}

Bending: Figure 6.4 shows compressive and tensile stresses in the trapezoidal section. Similar to channel section, tensile stress on the bottom is higher than the compressive stress for any given load level. Again, this is due to the fact that higher compressive area results in lower compressive stress, i.e., satisfying force equilibrium requirements. Failure occurred in the top of the member with failure compressive stress being 23,722 psi while the tensile stress was 45,049 psi. Overall average thickness of the section was 0.14 inches. This small thickness has contributed to the observed punching failure.

Tension tests on plates cut from trapezoidal section: Tension coupon plates cut from the trapezoidal section were tested with and without grips on the end. Lower strength values were obtained for the specimens tested without grips due to premature failure near the gripping areas. Tension failure at 39,124 psi for specimens with $13 / 4$ in grips was caused by bond problems at the interface between the composite and grip. This value was discarded and additional tests were conducted. Maximum tensile stress value of 82,897 psi was obtained for a grip length of $2 \frac{1}{2}$ in. This value can be considered as the absolute maximum tensile strength for this type of material. It can be seen that the section under bending did not reach its full tensile strength value due to premature failure. 


\subsection{Recycled ABS Box Sections}

\subsubsection{Test Results on Recycled ABS Box Section}

Bending: Tables 6.4 and 6.5 show the box dimensions and test results, respectively, under three and four point bending tests for FRP wrapped and unwrapped box sections. Both recycled and virgin polymer specimens were tested and evaluated. For all the test configurations, stiffness and ultimate strength values are shown in Table 6.6 and Figure 6.7, respectively. Punching failure occurred on the top member at loading points for recycled ABS specimens. Tensile and compressive stiffnesses were computed from the stress versus strain plots (Figure 6.7). Bending stresses were calculated as described for channel section. The distance from the neutral axis to strain gage location for non-wrapped specimens, wrapped specimens, and virgin vinylester non wrapped specimens were 0.487 in, 0.586 in, and 0.496 in, respectively. Moment of inertia of non wrapped section was the section was $I=0.044 \mathrm{in}^{4}$, for wrapped specimens was $\mathrm{I}=0.084 \mathrm{in}^{4}$ and for virgin Vinylester specimens $\mathrm{I}=0.056 \mathrm{in}^{4}$. Specimen cross sections are shown in Figure 6.6. Fiber content and dimensional properties of the specimens are summarized in Table 6.4. For 4-point bending tests maximum moment (between loads) was computed as follows:

$$
M=P a
$$

Where

$\mathrm{M}=$ Moment in the middle third zone

$\mathrm{P}=$ Applied load (Max load/2)

$\mathrm{a}=$ Distance from supports to each point of load application $=5$ in. 
Table 6.4 Fiber Volume Fraction and Dimensional Properties for ABS Box Sections

\begin{tabular}{|c|c|c|c|c|}
\hline Specimen & $\begin{array}{c}\text { Fiber } \\
\text { Volume } \\
\text { Fraction } \\
(\mathbf{\%})\end{array}$ & $\begin{array}{c}\text { Average } \\
\text { thickness }\end{array}$ & $\begin{array}{c}\text { Area of cross } \\
\text { section }\end{array}$ & $\begin{array}{c}\text { Moment of } \\
\text { Inertia }\end{array}$ \\
\hline $\begin{array}{c}\text { Non-wrapped } \\
\text { recycled }\end{array}$ & 25 & 0.10 & 0.348 & 0.0440 \\
\hline Wrapped recycled & 22 & 0.16 & 0.586 & 0.0837 \\
\hline $\begin{array}{c}\text { Non-wrapped } \\
\text { virgin* }\end{array}$ & 41 & 0.13 & 0.437 & 0.0562 \\
\hline
\end{tabular}

* Vinylester Specimen

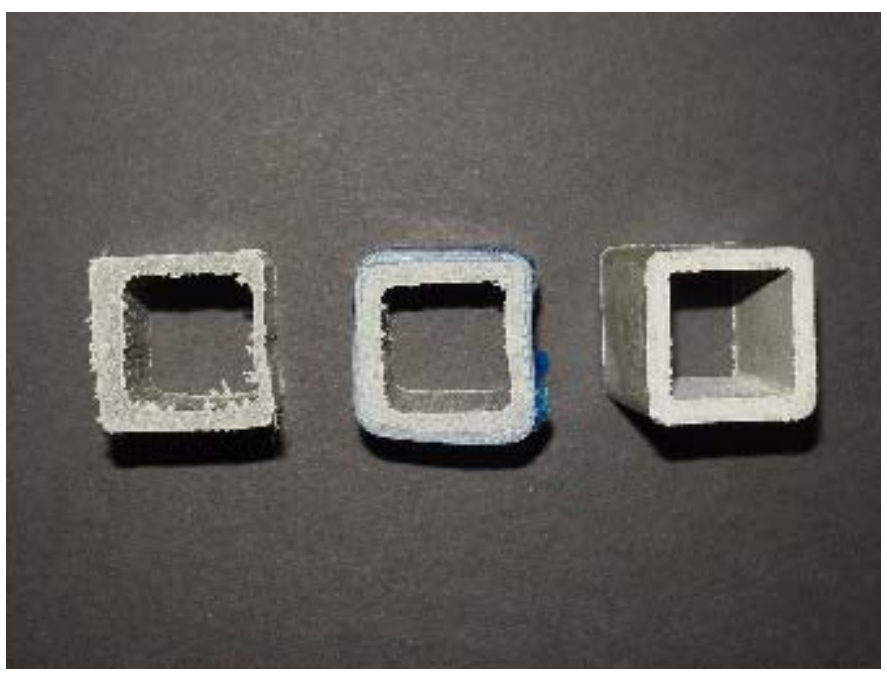

Figure 6.6 Box Sections (from Left to Right) Recycled ABS, Wrapped Recycled ABS and Virgin Vinylester

Table 6.5 Maximum Tensile and Compressive Stresses and Stiffnesses in Box

Sections

\begin{tabular}{|c|c|c|c|c|c|}
\hline Specimen & Type of test & $\begin{array}{c}\text { Tensile } \\
\text { Stiffness, } \\
\mathbf{E}(\mathbf{p s i})\end{array}$ & $\begin{array}{c}\text { Compressive } \\
\text { Stiffness, E } \\
\text { (psi) }\end{array}$ & $\begin{array}{c}\text { Maximum } \\
\text { Stress } \\
\text { (psi) }\end{array}$ & $\begin{array}{c}\text { Load on } \\
\text { loading point } \\
\text { at failure. } \\
\text { (lb) }\end{array}$ \\
\hline $\begin{array}{c}\text { Non-wrapped } \\
\text { recycled ABS }\end{array}$ & 3-point & $3.86 \times 10^{6}$ & $2.28 \times 10^{6}$ & 12,845 & 280 \\
\hline $\begin{array}{c}\text { Wrapped recycled } \\
\text { ABS }\end{array}$ & 3-point & $3.24 \times 10^{6}$ & $1.90 \times 10^{6}$ & 12,953 & 504 \\
\hline $\begin{array}{c}\text { Wrapped recycled } \\
\text { ABS }\end{array}$ & 4-point & $3.42 \times 10^{6}$ & $2.94 \times 10^{6}$ & 19,733 & 589 \\
\hline $\begin{array}{c}\text { Non-wrapped } \\
\text { virgin Vinylester }\end{array}$ & 3-point & $3.60 \times 10^{6}$ & $2.52 \times 10^{6}$ & 29,972 & 850 \\
\hline
\end{tabular}




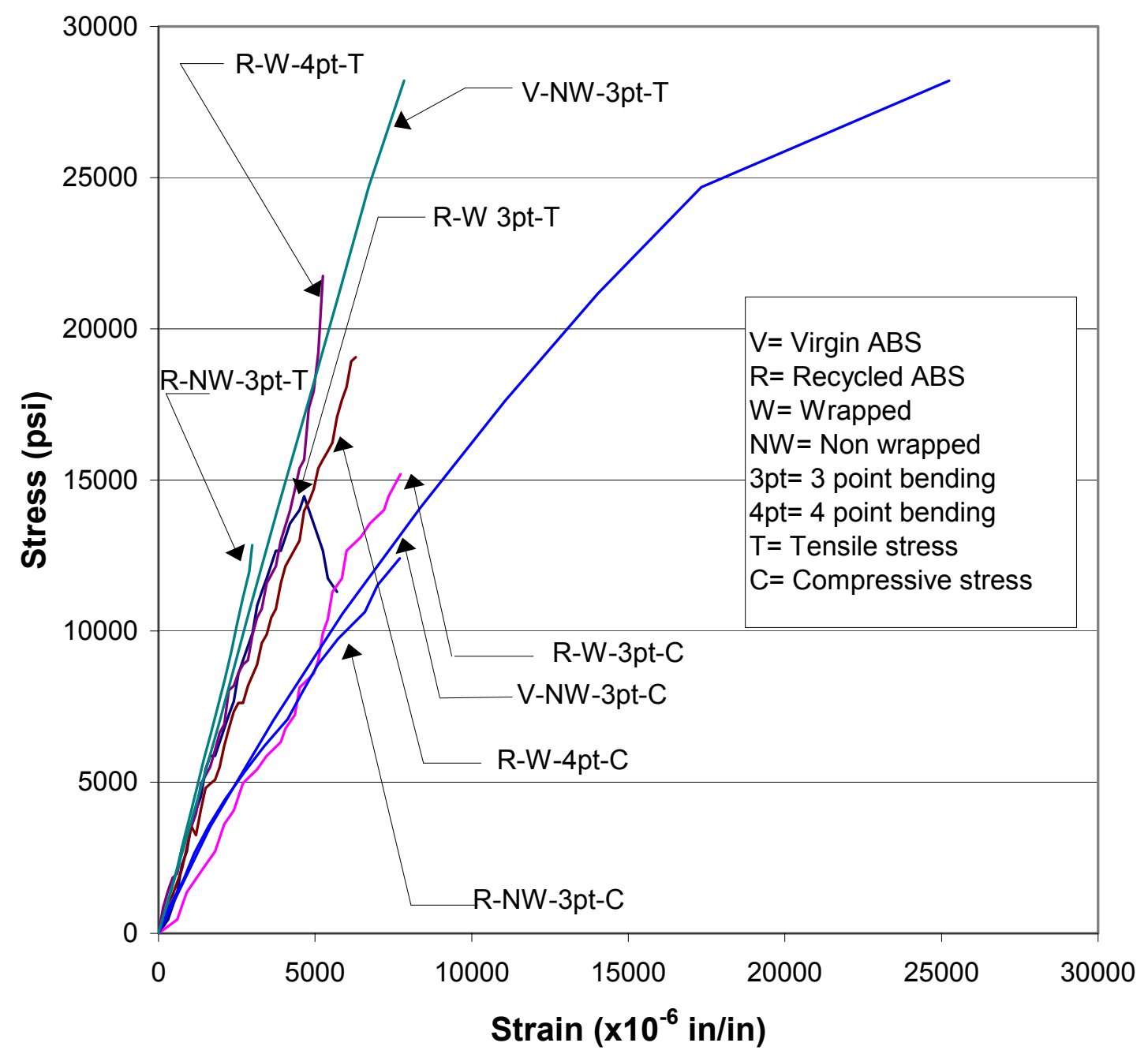

Note: Recycled ABS specimens failed in compression and punching

Virgin PP specimens failed in compression

Figure 6.7 Compressive and Tensile Bending Stresses in Recycled ABS Box Section Under Three and Four Point Bending Test

Tension: ABS box section as described in section 4.4.1 was tested under tension. Results of ultimate strength and stiffness are summarized in Table 6.6. Tensile stresses were calculated by dividing the applied force by the area of cross section. Stiffness was calculated from the stress versus strain curve (Figure 6.8). 
Table 6.6 Maximum Tensile Stress and Stiffness for Recycled ABS Box Section - Tension Test

\begin{tabular}{|c|c|}
\hline $\begin{array}{c}\text { Stiffness, } \mathbf{E} \\
(\mathbf{p s i})\end{array}$ & $\begin{array}{c}\text { Maximum Stress } \\
(\mathbf{p s i})\end{array}$ \\
\hline $3.21 \times 10^{6}$ & 19,960 \\
\hline
\end{tabular}

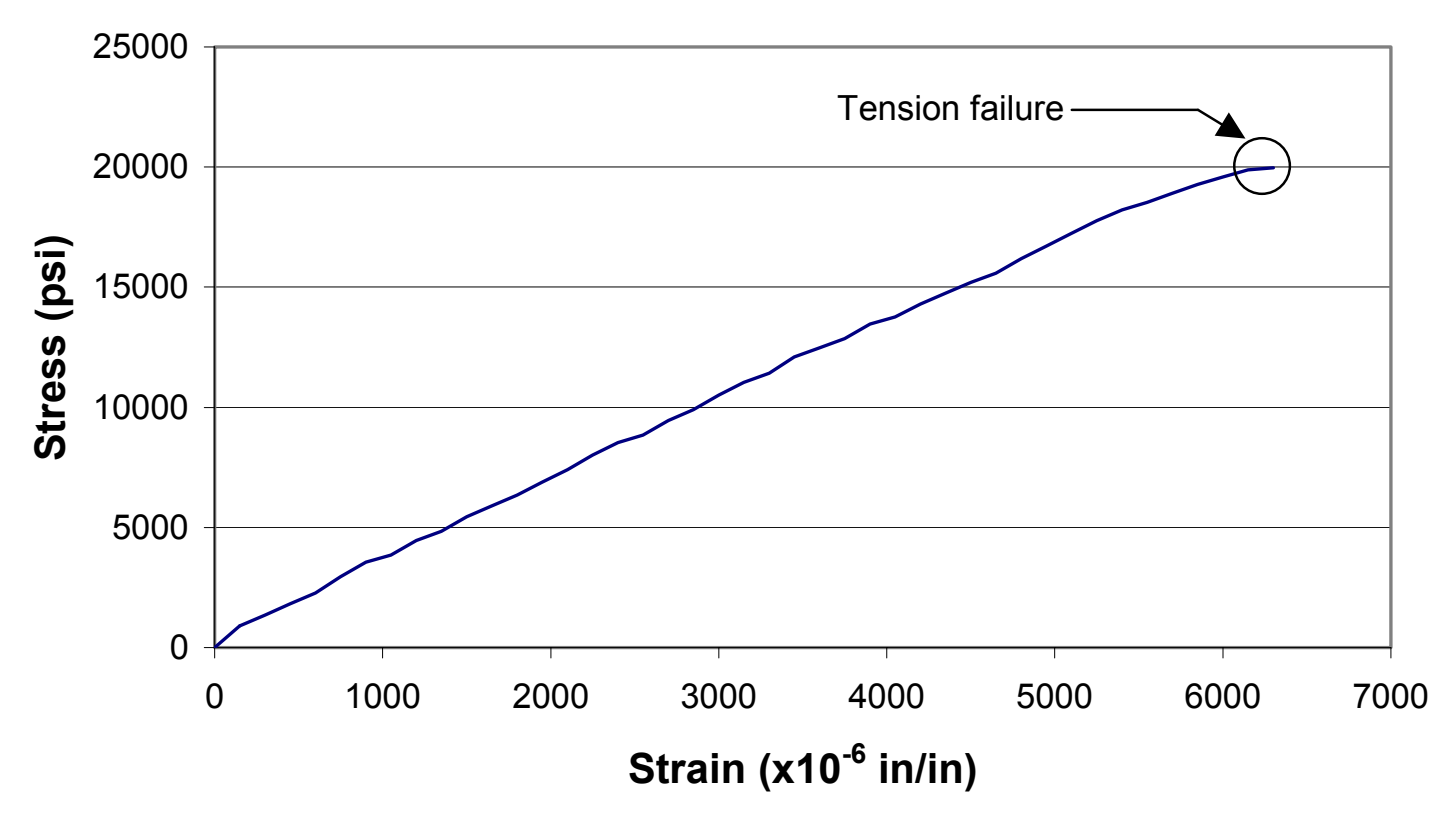

Figure 6.8 Tensile Stress vs. Strain for Tension Tests on Recycled ABS Box Section

\subsubsection{Analysis and Discussion of Test Results on Recycled ABS Box Section}

Bending: Tensile strains were observed to be higher than the compressive strains in box sections as seen in Figure 6.7. For the unwrapped specimens with a thickness of 0.10 in, ultimate stress under 3 -point bending was 12,845 psi. For wrapped section with a thickness of 0.16 in, ultimate stress under 3 point bending was 12,953 psi. Ultimate stress for 4-point bending test was 19,733 psi. For virgin box section with a thickness of 0.13 in, 3 point bending ultimate stress was 29,972 psi. 
Failure mode observed for the recycled specimens was a compression failure coupled with punching at the loading point. Both 3-point and 4-point bending stresses were well below the maximum stress values. However, punching ultimate stress under single location in 3-point bending was more critical than that under two locations in 4point bending. Test results indicate the effectiveness of wrap in terms of strength increase.

Virgin vinylester box specimen failed in compression at a much larger strain than the recycled ABS box sections. Higher strength of the virgin vinylester composite box section is attributed to the higher fiber volume fraction ( $41 \%$ versus $25 \%)$. It has to be noted that the fiber reinforced virgin vinylester specimen was an optimized design for a thermoset that can be looked as a final objective for recycled thermoplastic shapes.

From the bending results of three sections tested under this research, trapezoidal section showed better bending resistance and higher stress levels. Reinforcement at loading points in a prototype (i.e., junction of post and railing) is also a critical issue that needs to be properly accounted for when designing an actual size of a highway post or rail.

Tension: Ultimate tensile strength of 19,960 psi was obtained for the box section when tested as a single unit. Stiffness obtained was $3.21 \times 10^{6}$ psi. Tension value shows that box specimens under bending failed before they reached their maximum tensile stress capacity. 


\subsection{Plates from Recycled ABS Sheets}

\subsubsection{Test Results on Plates with Recycled ABS Sheets}

Bending: Table 6.7 shows the results for recycled ABS sheets tested under three point bending. Fiber volume fraction of $25 \%$ for recycled ABS sheets was determined by ignition test. Failure mode observed for short plates was longitudinal shearing delamination of the composite at an interlaminar shear stress of 874 psi. This low value is attributed to a poor wet out of the fabric during manufacturing process. For long plates, crushing on the compression surface of the plate was observed. Stiffnesses were computed from the stress versus strain plots (Figure 6.9). Bending stresses were calculated as described for the channel section. For short plates, distance from the neutral axis to top of the plate was 0.113 in, and moment of inertia was $\mathrm{I}=0.00072 \mathrm{in}^{4}$. For long plates, distance value was $0.100 \mathrm{in}$. and $\mathrm{I}=0.00101 \mathrm{in}^{4}$. Interlaminar shear stress due to bending was calculated as follows:

$$
\begin{aligned}
& \tau=\frac{V Q}{I} \\
& \text { Where } \\
& \tau=\text { Interlaminar shear stress } \\
& \mathrm{V}=\text { Shear force at point of stress calculation } \\
& \mathrm{Q}=\text { Moment of area about centroid of shear area } \\
& \mathrm{I}=\text { Moment of inertia }
\end{aligned}
$$$$
\text { For a rectangular section, the interlaminar shear stress can be calculated as: }
$$$$
\tau=1.5 \frac{V}{A}
$$ 
Where

$\mathrm{V}=0.5 \mathrm{P}=$ Shear at midspan for a simply supported beam under point load $\mathrm{P}$

$\mathrm{A}=$ total area

Table 6.7 Maximum Tensile and Compressive Stresses and Stiffnesses in Recycled ABS Plates - Three Point Bending Test

\begin{tabular}{|l|c|c|c|c|c|}
\hline Specimen & $\begin{array}{c}\text { Tensile } \\
\text { Stiffness, E } \\
\text { (psi) }\end{array}$ & $\begin{array}{c}\text { Compressive } \\
\text { Stiffness, E } \\
\text { (psi) }\end{array}$ & $\begin{array}{c}\text { Maximum } \\
\text { stress } \\
\text { (psi) }\end{array}$ & $\begin{array}{c}\text { Load at } \\
\text { failure } \\
\text { (lb) }\end{array}$ & $\begin{array}{c}\text { Interlaminar } \\
\text { shear stress } \\
\text { (psi) }\end{array}$ \\
\hline Short plates & $2.59 \times 10^{6}$ & $2.33 \times 10^{6}$ & 27,991 & 197 & $874^{*}$ \\
\hline Long plates & $3.025 \times 10^{6}$ & $2.91 \times 10^{6}$ & 39,518 & 160 & $400^{* *}$ \\
\hline
\end{tabular}

* Separation of fabric from resin (interlaminar shear failure)

** Not an interlaminar shear failure

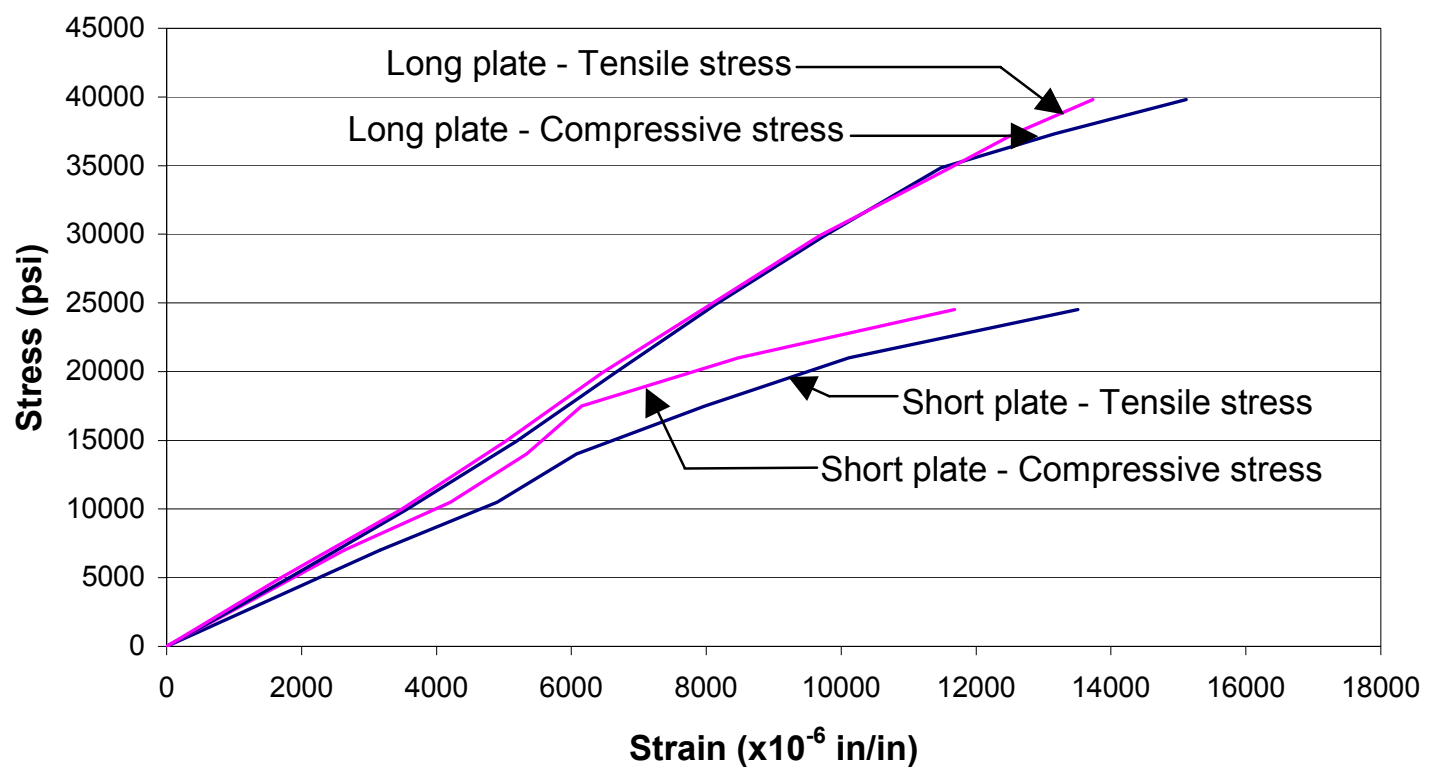

Note: Long plates failed in compression - Short plates failed in interlaminar shear

Figure 6.9 Tensile and Compressive Bending Stress in Recycled ABS Plates and Under Three Point Bending Test

Tension: Results of ultimate strength and stiffness on ABS plates cut from sheets are provided in Table 6.8. Stiffnesses were computed from the stress versus strain curves (Figure 6.10). Tensile stresses were computed as described for plates cut from trapezoidal section (Section 6.3.1) 
Table 6.8 Maximum Tensile Stress and Stiffness on Plates from ABS Sheets Tension Test

\begin{tabular}{|c|c|}
\hline $\begin{array}{c}\text { Stiffness, } \mathbf{E} \\
\text { (psi) }\end{array}$ & $\begin{array}{c}\text { Maximum Stress } \\
\text { (psi) }\end{array}$ \\
\hline $2.68 \times 10^{6}$ & 21,950 \\
\hline
\end{tabular}

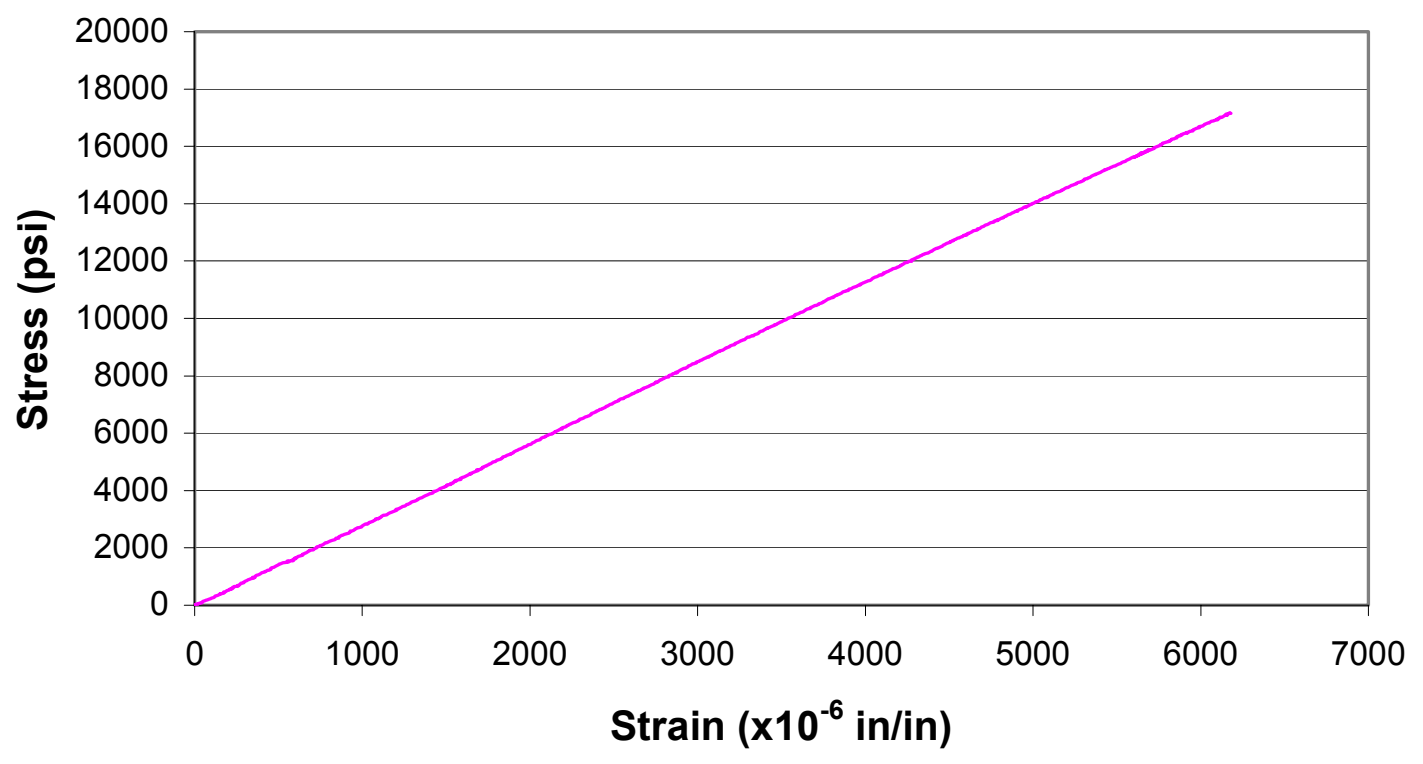

Note: Failure point not shown

Figure 6.10 Linear Portion of Stress vs. Strain for Tension Tests on ABS Plates from Recycled ABS Sheets

Compression: Small rectangular coupons cut from ABS sheets with cross section of $0.5 " \mathrm{n} 3 / 8 \mathrm{"}$ and height of $0.5 \mathrm{"}$ as per ASTM D695-91 were tested under compression. Results are shown in Table 6.9. Stiffnesses were computed from the stress versus strain curves (Figure 6.11). Compressive stresses were computed as follows:

$$
\sigma=\frac{P}{A}
$$


Where

$\sigma=$ Compressive stress

$\mathrm{P}=$ Applied load

$A=$ Area of cross section

Table 6.9 Maximum Compressive Stress and Stiffness on Plates from ABS Sheets

\begin{tabular}{|c|c|}
\hline $\begin{array}{c}\text { Stiffness, E } \\
\text { (psi) }\end{array}$ & $\begin{array}{c}\text { Maximum Stress } \\
\text { (psi) }\end{array}$ \\
\hline $3.58 \times 10^{6}$ & 6,900 \\
\hline
\end{tabular}

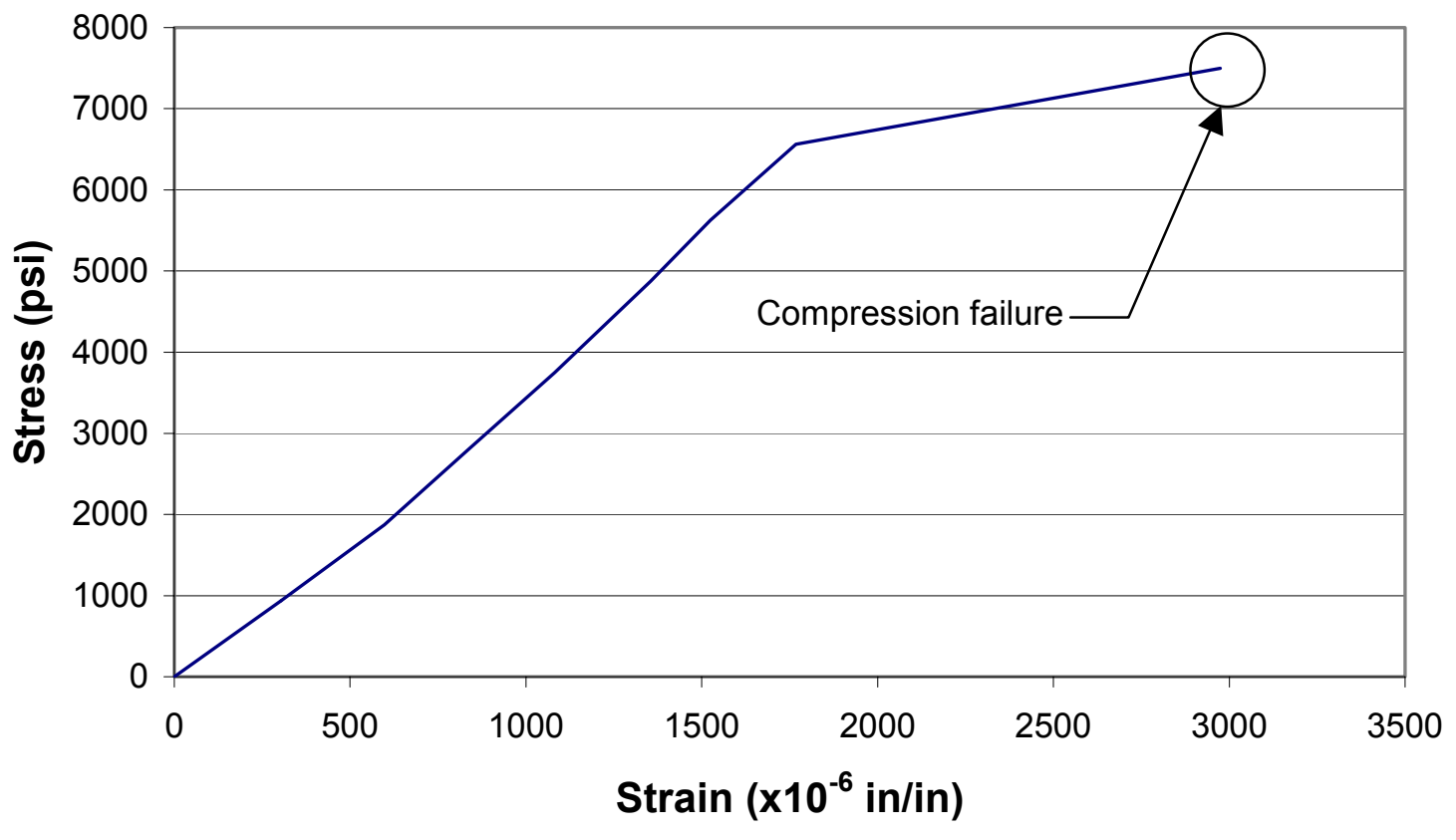

Figure 6.11 Compressive Stress vs. Strain for Compression Tests on Plates from Recycled ABS Sheets 


\subsubsection{Analysis and Discussion of Plate Test Results from Recycled ABS Sheets}

Bending: Ultimate bending strength of recycled ABS plates with a thickness of 3/8 in. was 39,518 psi. . Failure consisted of crushing on the compression surface, which is different from the observed punching of the other shapes described before. Short plate mode of failure was shear delamination at a maximum interlaminar shear stress of 874 psi, indicating a poor wet out of the fabric during manufacturing process.

Tension: Maximum average tensile strength of 21,950 was observed for ABS plates cut from sheets.

Compression: Under compression, specimen split open, showing buckling delamination and thus a low compression strength value of 6,900 psi was obtained. Most likely, delamination was caused because of the small thickness of $3 / 8$ of the specimens tested. It should be noted that ASTM recommends a thickness of at least $1 / 2 \mathrm{in}$. In addition, some fiber wet out problems were also noted.

\subsection{Strips from Recycled ABS Belt-Type Specimens}

\subsubsection{Test Results on Strips from Recycled ABS Belt-Type Specimens}

Tension: Three different belt-type specimens were received for testing. Results of tension tests on strips cut from the belt type specimens are shown in Table 6.10. Stiffnesses were computed from the slope of the stress vs. strain curves (Figure 6.12). Tensile stresses were computed as described for plates from trapezoidal section (Section 6.3.1). 
Table 6.10 Maximum Stress and Stiffness of Strips from ABS Belt -Type Specimens - Tension Test

\begin{tabular}{|c|c|c|c|}
\hline Type of Specimen & $\begin{array}{c}\text { Fiber volume } \\
\text { fraction } \\
\mathbf{( \% )}\end{array}$ & Stiffness, E & Maximum Stress \\
\hline A & 20 & (psi) & (psi) \\
\hline B & 24 & $2.92 \times 10^{6}$ & 29,800 \\
\hline C & 19 & $2.65 \times 10^{6}$ & 37,150 \\
\hline
\end{tabular}

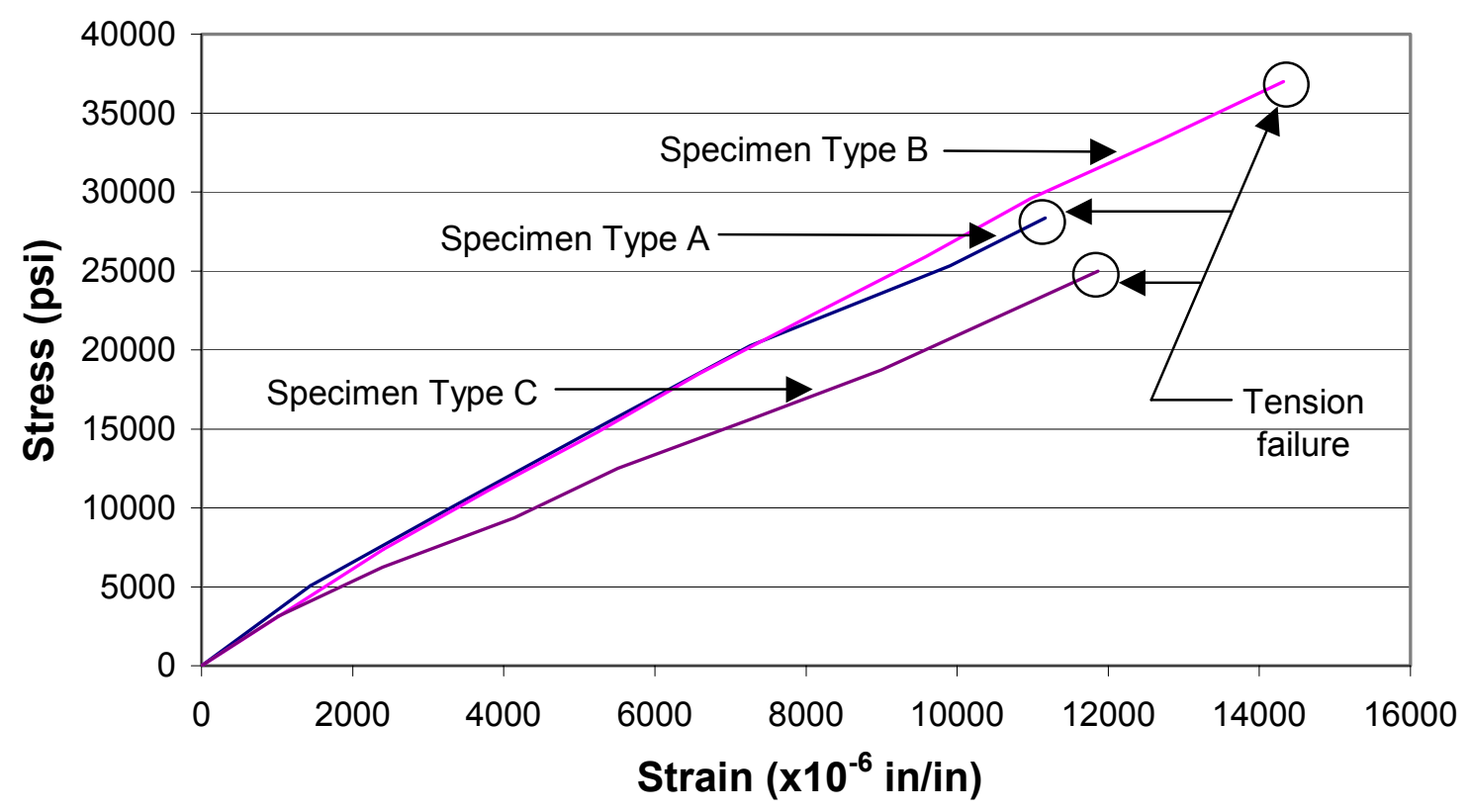

Figure 6.12 Tensile Stress vs. Strain of Recycled ABS Strips from Belt-Type Specimens

\subsubsection{Analysis and Discussion of Test Results on Strips from Recycled ABS Belt-} Type Specimens

Tension: Tensile strength of specimens varied from 24,450 psi for type C specimens up to 37,150 psi for Type B specimens. Type A specimens showed an ultimate strength of 29,800 psi. Lowest stiffness of $2.09 \times 10^{6}$ psi was obtained for Type C specimens as compared to $2.65 \times 10^{6}$ psi for Type B specimens and $2.92 \times 10^{6}$ psi for Type A samples. 


\subsection{Compression Tests of Rubber-Wood Offset Block Model}

\subsubsection{Results on Compression Test of Rubber-Wood Offset Block Model}

A maximum load of 5,250 $\mathrm{lb}$ was applied to the joint block. Test was stopped because of excessive deformation (buckling) of the rubber strips. This buckling was due to the fact that the strips were tested about weak axis with a high slenderness ratio. However, when compression tests were performed on the rubber block (3" $\mathrm{x} 3$ ") compressive stress in excess of $50 \mathrm{ksi}$ was noted. Compressive stress on the block at this point was 595 psi.

\subsubsection{Analysis and Discussion of Compression Test of Rubber-Wood Offset Block Model}

As expected, block without lateral support buckled at a very low applied load (Figure 4.45). Tests showed good performance of the materials (rubber and wood). However, applied load was well below the maximum value for individual components of the block. Interaction of the parts was satisfactory, none of the parts suffered any damage. In addition, strips were not placed one over the other in a stack, which would have enormously increased the total compressive load.

\subsection{Tests on Wood from Rubber - Wood Block Model}

\subsubsection{Results of Tests on Wood from Rubber - Wood Block Model}

In order to evaluate the strength of wood used for the rubber-wood prototype and compare to polymer strength, compression and impact tests were carried out.

Compression parallel to grain: Ultimate strength and stiffness of wood samples tested in compression with load applied parallel to grain are shown in Table 6.11. 
Stiffnesses were computed from the slope of the stress vs. strain curves (Figure 6.13).

Compressive stresses parallel to grain were computed as follows:

$\sigma=\frac{P}{A}$

Where

$\sigma=$ Compressive stress

$\mathrm{P}=$ Applied load

$A=$ Area of cross section parallel to grain

Table 6.11 Maximum Compressive Stress and Stiffness of Wooden Blocks from

Rubber - Wood Block Model - Compression Parallel to Grain Test

\begin{tabular}{|c|c|}
\hline $\begin{array}{c}\text { Stiffness, } \mathbf{E} \\
(\mathbf{p s i})\end{array}$ & $\begin{array}{c}\text { Maximum Stress } \\
(\mathbf{p s i})\end{array}$ \\
\hline $1.63 \times 10^{6}$ & 8,399 \\
\hline
\end{tabular}

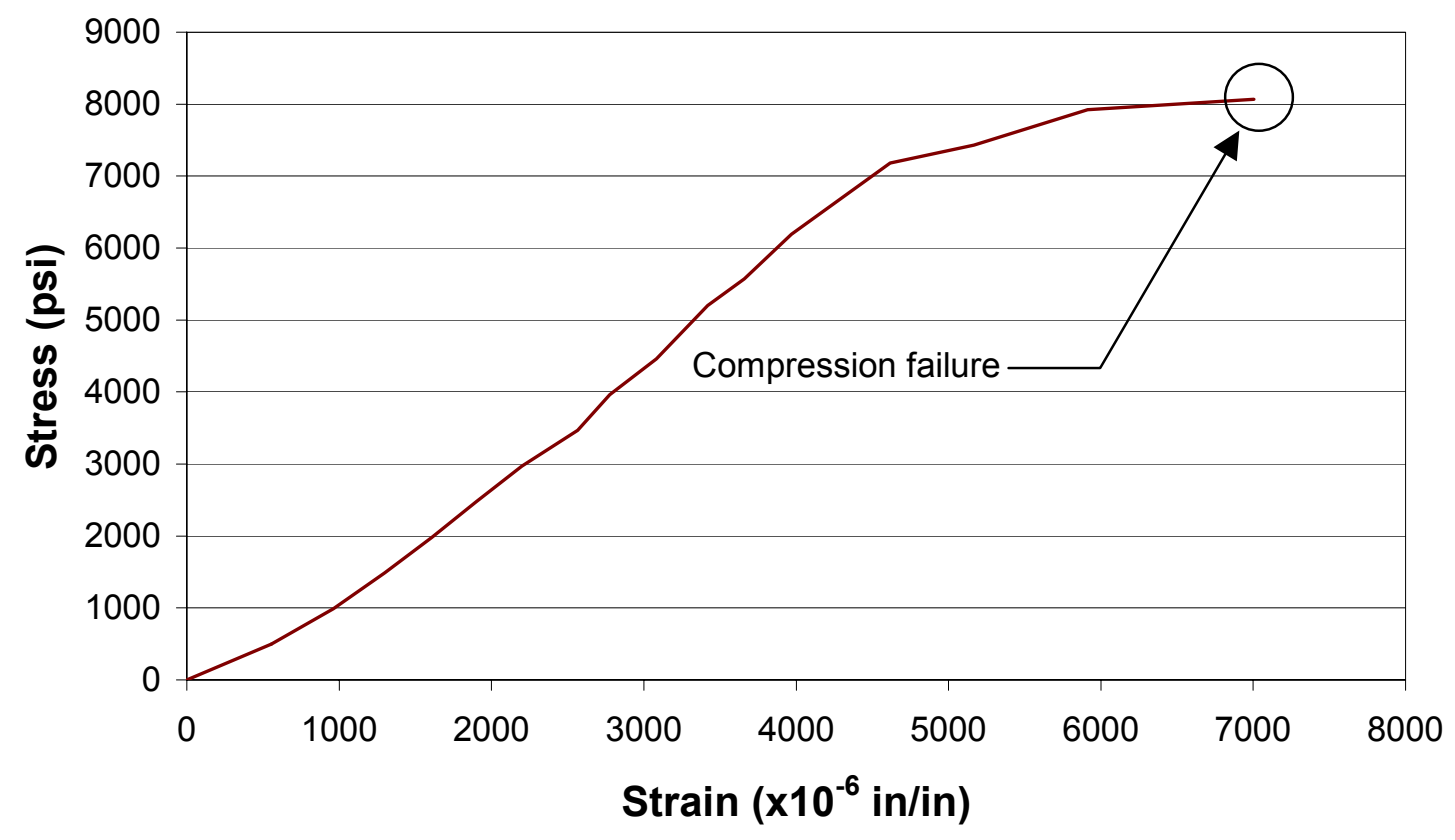

Figure 6.13 Compressive Stress vs. Strain for Compression Parallel to Grain Tests on Wooden Blocks from Rubber - Wood Block Model 
Compression perpendicular to grain: Ultimate strength and stiffness of wood samples tested in compression with load applied perpendicular to grain are shown in Table 6.12. Stiffnesses were computed from the stress versus strain curves (Figure 6.14). Compressive stresses parallel to grain were computed as follows:

$\sigma=\frac{P}{A}$

Where

$\sigma=$ Compressive stress

$\mathrm{P}=$ Applied load

$A=$ Area of cross section perpendicular to grain

Table 6.12 Maximum Compressive Stress and Stiffness of Wood Blocks from

Rubber - Wood Block Model - Compression Perpendicular to Grain Test

\begin{tabular}{|c|c|}
\hline $\begin{array}{c}\text { Stiffness, } \mathbf{E} \\
(\mathbf{p s i})\end{array}$ & $\begin{array}{c}\text { Maximum Stress } \\
(\mathbf{p s i})\end{array}$ \\
\hline $0.197 \times 10^{6}$ & 1,472 \\
\hline
\end{tabular}

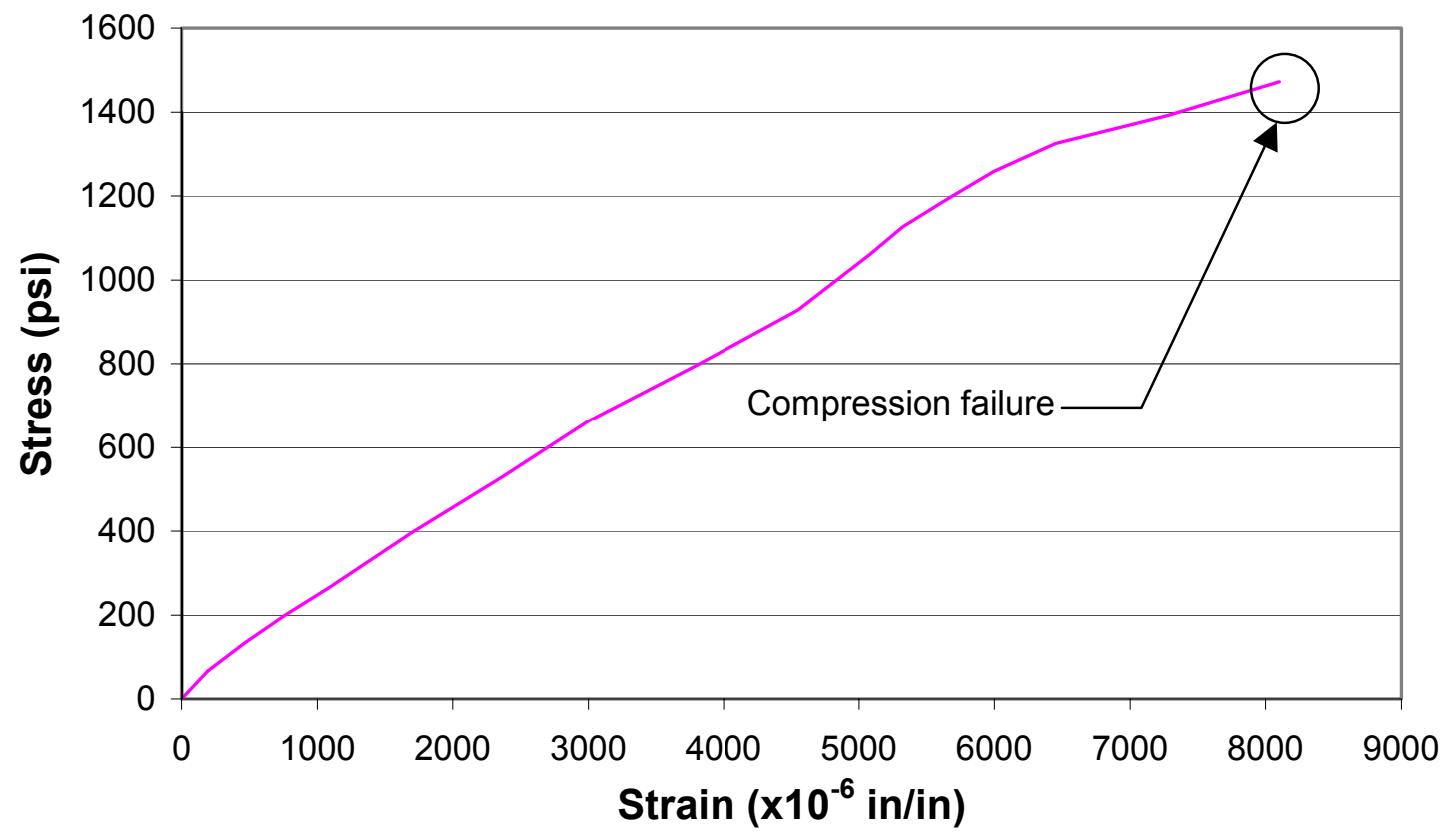

Figure 6.14 Stress vs. Strain for Compression Perpendicular to Grain Tests on Wooden Blocks from Rubber - Wood Block Model 
Impact: Average value of Izod impact strength obtained from the notched specimens was $1.82 \mathrm{ft}-\mathrm{lb} / \mathrm{in}$. Values varied from 0.07 to $4.31 \mathrm{ft}-\mathrm{lb} / \mathrm{in}$. Impact strength was calculated as follows:

$$
S=\frac{I}{b}
$$

Where

$S=$ Impact strength

$\mathrm{I}=$ Wind and friction corrected impact strength from Izod impact apparatus $\mathrm{b}=$ Thickness of the specimen

\subsubsection{Analysis and Discussion of Tests on Wood from Rubber - Wood Block Model}

Compression: As expected, compression resistance parallel to grain of the wood used for the rubber block is 5.7 times greater than compression perpendicular to grain. Stiffness parallel to grain was 8.3 times greater than the stiffness perpendicular to grain. Values obtained from these tests will be used only as a reference for later comparison to polymer composite offset blocks.

Impact: Lower values for impact resistance were obtained for wood samples than virgin and recycled polymers such as $\mathrm{ABS}$ or PC. Figure 6.15 shows a comparison of impact strength between wood, ABS and PC (both virgin and recycled). 


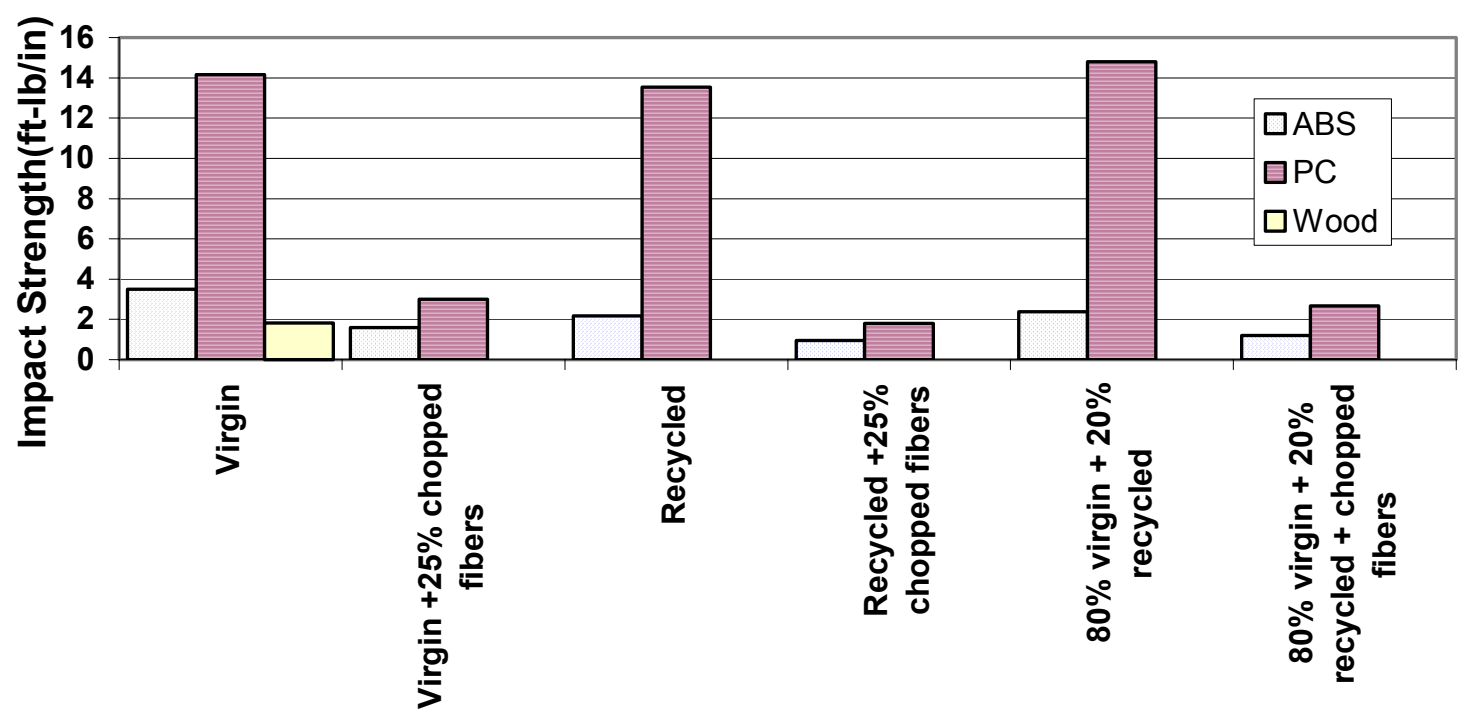

Type of Composite/Resin

Figure 6.15 Comparison of Impact Strength Between Virgin and Combinations of Recycled ABS and PC, and Wood

As shown in Figure 6.15, wood impact strength of $1.82 \mathrm{ft}-\mathrm{lb} / \mathrm{in}$ is about 8 times less than virgin PC (14.15 ft-lb/in) and about 2 times less than virgin ABS (3.19 ft-lb/in). When compared to recycled and blended (virgin/recycled) resins, wood impact strength is at least 7.4 times less than that of $\mathrm{PC}$ and at least 1.2 times less than that of $\mathrm{ABS}$ specimens. Impact strength in all three kinds of PC specimens with fiber addition was equal to or higher than impact stress in wood (1.81 to $3.01 \mathrm{ft}-1 \mathrm{~b} / \mathrm{in}$ vs. $1.82 \mathrm{ft}-\mathrm{lb} / \mathrm{in})$. However impact strength in wood was higher than that in chopped fiber reinforced ABS specimens ( 0.96 to $1.59 \mathrm{ft}-\mathrm{lb} / \mathrm{in}$ vs. $1.82 \mathrm{ft}-\mathrm{lb} / \mathrm{in})$. This gives us an idea on the efficiency of some polymer composites for future impact strength designs, which is one of the main concerns when designing guardrail systems. 


\subsection{Bond Strength Test on Rubber-ABS Interface}

As part of the development of an offset block for highway guardrail systems, a number of tests were performed to characterize the bond strength between rubber tire surface and molded recycled ABS. In this section test results are presented and discussed.

\subsubsection{Results of Bond Strength Test on Rubber-ABS Interface}

Bond pull out tests were conducted with and without applying primer to the surface of rubber prior to molding the recycled ABS sample to obtain good interfacial contact between ABS and rubber. Figure 6.16 shows stress vs. deflection during bond pull out test conducted for different curing-bonding configurations. Test results are summarized in Table 6.13 and illustrated in Figure 6.17. Bond stress was calculated as follows:

$$
\tau=\frac{P}{A}
$$

Where

$\tau=$ Bond stress

$\mathrm{P}=$ Pulling load

$\mathrm{A}=$ Bond area (rubber surface in contact with $\mathrm{ABS}$ ) 


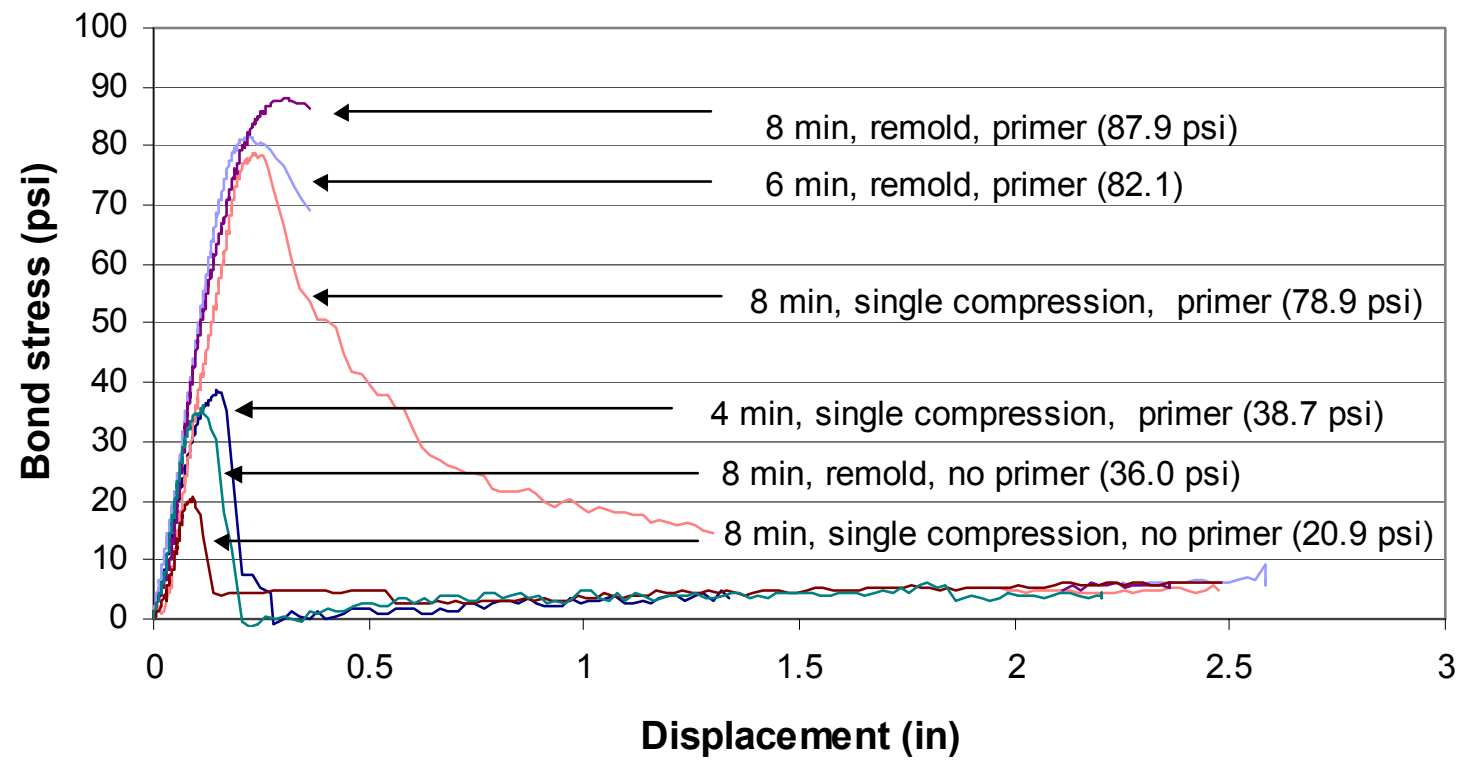

Note: Ultimate bond stress values are indicated in parenthesis

Figure 6.16 Bond Stress vs. Deflection for Pull Out Tests on ABS - Rubber Interface

Table 6.13 Bond Strength Test Results ABS-Rubber Interface

\begin{tabular}{|c|c|c|c|}
\hline \multirow{3}{*}{ Bond mechanism } & Molding Process & Molding Time & Ultimate bond \\
& & & stress (psi) \\
\hline \multirow{2}{*}{ No primer } & Single compression & 8 & 20.9 \\
\cline { 2 - 4 } & Remolded & 8 & 36.0 \\
\hline \multirow{3}{*}{ Primer } & Single compression & 4 & 38.7 \\
\cline { 2 - 4 } & Remolded & 6 & 82.1 \\
\cline { 2 - 4 } & Single compression & 8 & 87.9 \\
\cline { 2 - 4 } & Remolded & 8 & 87.9 \\
\hline
\end{tabular}




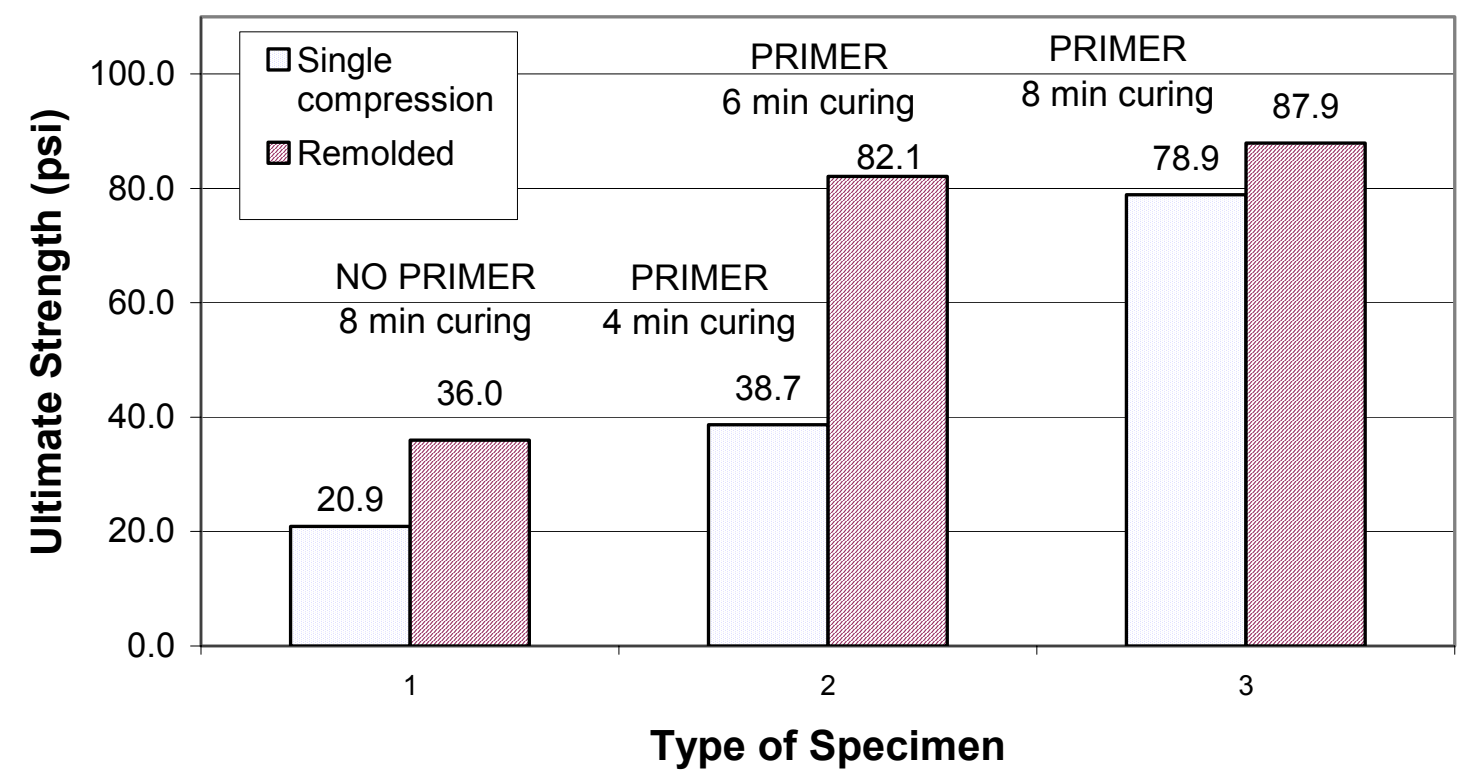

Figure 6.17 Results of Bond Strength Tests on ABS - Rubber Interface With and Without Primer for Different Molding (Curing) Duration

\subsubsection{Analysis and Discussion of Bond Strength Test on Rubber-ABS Interface}

From the pull out tests, it was observed that:

- Better bond stress between rubber and ABS interface was obtained when the specimens were compression molded for 8 minutes. Specimens with 4 minute cure showed bond strength of $38.7 \mathrm{psi}$ as compared to $78.9 \mathrm{psi}$ for specimens with 8 minute cure. Specimens cured for 6 minutes showed bond strength of 82.1 psi as compared to 87.9 psi for specimens cured for 8 minutes

- Use of primer increased the bond strength. Specimens without primer had bond strength of 20.9 psi as compared to 78.9 psi for a single molding cycle with 8 minute curing. For specimens with primer, samples cured for just 4 
minutes showed bond strength of 38.7 psi which is $85 \%$ higher than the bond strength (20.9 psi ) obtained for the 8 minute cured sample without primer.

- Remolding increased the bond strength significantly. Specimens without primer showed an increase from 20.9 psi to $36.0 \mathrm{psi}$ ( $72 \%$ increase) in bond strength when remolded. Samples with primer showed an increase from 78.9 psi to 87.9 psi in bond strength when remolded.

It can be concluded that the optimum bond strength can be obtained when primer is applied to the rubber surface and specimens are remolded. However, taking into account that under a mass production operation, remolding of products will double the processing time and eventually increase the cost, remolding of samples should be avoided if possible. Remolding may increase the bond strength by up to $10 \%$. 


\section{CHAPTER 7}

\section{SUMMARY, CONCLUSIONS AND RECOMMENDATIONS FOR COUPON SPECIMENS FROM CONDITIONED RECYCLED THERMOPLASTICS}

\subsection{Introduction}

Aging (conditioning) of fiber reinforced (chopped) and non- fiber reinforced ABS and PC thermoplastics specimens manufactured from virgin and recycled electronic shredder residue (ESR) was carried out for a period of 18 months which is equivalent to more than 60 years of natural weathering. Based on the accelerated aging study by Vijay and GangaRao (1999), glass fiber reinforced sand coated bars subjected to salt and alkaline conditioning indicated maximum strength reductions of $21.9 \%$ and $37.5 \%$, respectively, over 30 months duration. Based on the most severe alkaline conditioning, the authors predict the serviceability of those GFRP bars with urethane modified vinylester (thermoset) resins to be at least 60 years with $20 \%$ sustained stress. In our experiments, based on $30 \%$ maximum strength reduction without sustained stress over 18 months, it would be safe to assume at least 60 years of service life for products made of recycled polymers. However, careful considerations has to be given to type of fibers, purity of resins, $\mathrm{pH}$, and sustained stress values, and quality control in manufacturing, before applying such conclusions to a particular recycled product. After conditioning, the coupon specimens were tested to evaluate tension, bending, compression, impact, and hardness properties. 


\subsection{Summary of Tension Test Results}

Specimens without chopped fibers conditioned for 18 months:

- Maximum reduction in tensile strength without chopped fibers was $6.1 \%$ and 9.6\% for $\mathrm{ABS}$ and $\mathrm{PC}$ specimens, respectively. Average maximum tensile strength reduction in those $\mathrm{ABS}$ and $\mathrm{PC}$ specimens during the aging period was $5.7 \%$ and $7.9 \%$, respectively.

- Maximum reduction in tensile stiffness of ABS specimens without chopped fibers was 3.3\%. Average maximum reduction in those ABS specimens during the aging period was $2.0 \%$. No reductions (some gains) in tensile stiffness were observed for PC specimens without chopped fibers.

Specimens with chopped fibers conditioned for 18 months:

- Maximum reduction in tensile strength with chopped fibers was $13.6 \%$ and $28.4 \%$ for $\mathrm{ABS}$ and $\mathrm{PC}$ specimens, respectively. Average maximum tensile strength reduction in those ABS and PC specimens was $11.1 \%$ and $28.2 \%$.

- Maximum reduction in tensile stiffness with chopped fibers was $5.7 \%$ and $2.9 \%$ for $\mathrm{ABS}$ and $\mathrm{PC}$ specimens, respectively. Average maximum tensile strength reduction in those $\mathrm{ABS}$ and $\mathrm{PC}$ specimens during aging period was $5.7 \%$ and $2.5 \%$, respectively.

\subsection{Summary of Bending Test Results}

Specimens without chopped fibers conditioned for 18 months:

- Maximum reduction in bending strength without chopped fibers was $25.9 \%$ and $26.8 \%$ for $\mathrm{ABS}$ and $\mathrm{PC}$ specimens, respectively. Average maximum 
bending strength reduction in those $\mathrm{ABS}$ and $\mathrm{PC}$ specimens during the aging period was $17.4 \%$ and $18.94 \%$, respectively.

- Maximum reduction in bending stiffness of ABS specimens was $16.5 \%$ for specimens without chopped fibers. Average maximum reduction in the aging period was $13.0 \%$. No reductions in bending stiffness of PC were observed for specimens without chopped fibers during the aging period.

Specimens with chopped fibers conditioned for 18 months:

- Maximum reduction in bending strength with chopped fibers was $30.9 \%$ and $26.2 \%$ for $\mathrm{ABS}$ and $\mathrm{PC}$ specimens, respectively. Average maximum bending strength reduction in those $\mathrm{ABS}$ and $\mathrm{PC}$ specimens during the aging period was $26.4 \%$ and $22.1 \%$, respectively.

- Maximum reduction in bending stiffness of ABS specimens was $17.4 \%$ for specimens with chopped fibers. Average maximum reduction in the aging period was $13.3 \%$. No reductions in bending stiffness of PC were observed for specimens with chopped fibers during the aging period.

\subsection{Summary of Compression Test Results}

Specimens without chopped fibers conditioned for 18 months:

- Maximum reduction in compressive strength without chopped fibers was $8.7 \%$ and $5.6 \%$ for $\mathrm{ABS}$ and $\mathrm{PC}$ specimens, respectively. Average maximum compressive strength in those ABS and PC specimens during the aging period was $8.0 \%$ and $3.8 \%$, respectively. 
- Maximum reduction in compressive stiffness of ABS specimens was $19.5 \%$ for specimens without chopped fibers. Average maximum reduction in the aging period was $19.5 \%$. No reductions in compressive stiffness of PC were observed for specimens without chopped fibers during the aging period.

Specimens with chopped fibers conditioned for 18 months:

- Maximum reduction in compressive strength with chopped fibers was $32.4 \%$ and $44.7 \%$ for $\mathrm{ABS}$ and $\mathrm{PC}$ specimens, respectively. Average maximum compressive strength reduction in those $\mathrm{ABS}$ and $\mathrm{PC}$ specimens during the aging period was $26.5 \%$ and $41.7 \%$, respectively.

- Maximum reduction in compressive stiffness of ABS specimens was $34.8 \%$ for specimens with chopped fibers. Average maximum reduction in the aging period was $32.63 \%$. No reductions in compressive stiffness of PC were observed for specimens with chopped fibers during the aging period.

\subsection{Summary of Impact Test Results}

Specimens without chopped fibers conditioned for 18 months:

- Maximum reduction in impact strength without chopped fibers was $2.3 \%$, $61.6 \%$ and $38.8 \%$ for virgin, $100 \%$ recycled, and blended (20\% virgin / $80 \%$ recycled) ABS specimens, respectively.

- Maximum reduction in impact strength of PC specimens was $1.5 \%$ for specimens without chopped fibers. Average maximum reduction in the aging period was $1.5 \%$. 
Specimens with chopped fibers conditioned for 18 months:

- Maximum reduction in impact strength of ABS specimens was $27.2 \%$ for specimens with chopped fibers. Average maximum reduction in the aging period was $23.5 \%$.

- Maximum reduction in impact strength of PC specimens was $56.2 \%$ for specimens with chopped fibers. Average maximum reduction in the aging period was $54.9 \%$.

\subsection{Summary of Hardness Test Results}

Specimens without chopped fibers conditioned for 18 months:

- Maximum reduction in hardness index of $\mathrm{ABS}$ specimens was 3.6\% for specimens without chopped fibers. Average maximum reduction in the aging period was $2.2 \%$. No reductions in hardness index of PC were observed for specimens without chopped fibers during the aging period.

Specimens with chopped fibers conditioned for 18 months:

- Maximum reduction in hardness index of ABS specimens was $5.4 \%$ for specimens with chopped fibers. Average maximum reduction in the aging period was $4.2 \%$. No reductions in hardness index of PC were observed for specimens with chopped fibers during the aging period.

\subsection{Summary of Creep Test Results}

Specimens without chopped fibers conditioned for 18 months:

- At $20 \%$ sustained load, average creep coefficient for ABS specimens was 1.81 and 0.50 for PC samples. 
- At $50 \%$ sustained loading, ABS specimens failed before 44 days. On the other hand, average creep coefficient for PC samples was 0.81 .

Specimens with chopped fibers conditioned for 18 months:

- At $20 \%$ sustained loading, average creep coefficient for ABS specimens was 0.83 and 0.36 for PC samples.

- At 50\% sustained loading, average creep coefficient for ABS specimens was 1.82 and 0.44 for PC samples.

\subsection{Conclusions and Recommendations}

- In general, virgin polymers were less susceptible to harsh environment as compared to $100 \%$ recycled and blend of virgin and recycled resins.

- Among tensile, bending, and compressive properties, compressive strength reductions were higher in terms of both strength and stiffness.

- Reductions in tensile strength and stiffness were less than those in bending strength and stiffness.

- Impact strength was most sensitive to aging among all the mechanical properties evaluated. For every type of ABS and PC specimens, a decrease in impact strength was observed varying from $1.5 \%$ (PC without fibers) to $61.6 \%$ (100\% recycled ABS without fibers). Variability can be attributed in part to the random orientation of chopped fibers.

- Hardness properties of both ABS and PC specimens were not significantly affected in many of the specimens after aging. 
- Reductions observed in tensile, bending and compressive strength and stiffness of PC specimens were generally higher than those in tensile, bending and compressive strength and stiffness of ABS specimens.

- Based on test results, recycled thermoplastic polymers with no fiber addition were found to retain at least $92 \%$ of their tensile strength, $81 \%$ of their bending strength and $92 \%$ of their compressive strength when conditioned up to 18 months under harsh environment.

- Based on test results, chopped fiber reinforced recycled thermoplastic polymers were found to retain at least $71 \%$ of their tensile strength, $73 \%$ of their bending strength and $58 \%$ of their compressive strength when conditioned under harsh environment.

- The aging process effect on stiffness of PC specimens with and without fibers was not significant. However stiffness of ABS specimens was more sensitive to aging under harsh environment than PC specimens.

- Based on test results, recycled thermoplastic polymers with no fiber addition were found to retain at least $98 \%$ of their tensile stiffness, $87 \%$ of their bending stiffness, and $80 \%$ of their compressive stiffness when conditioned under harsh environment.

- Based on test results, chopped fiber reinforced recycled thermoplastic polymers were found to retain at least $96 \%$ of their tensile stiffness, $86 \%$ of their bending stiffness and $64 \%$ of their compressive stiffness when conditioned under harsh environment. 
- Higher reductions in strength and stiffness of specimens with chopped fibers are due to existence of moisture "channels" that facilitate moisture diffusion along fiber/resin interface resulting in higher deterioration in specimens with chopped fibers.

- Use of continuous fibers or proper surface coating to seal off the open interfaces is expected to reduce moisture related degradation problems.

- Based on the test results, it is concluded that the recycled polymers can be appropriately used with chopped or continuous fibers for long term structural or automobile applications with suitable knock-down factors.

- Further investigation using continuous fibers is highly recommended in order to evaluate a possible mitigation of aging effects and reduction on variability of test results.

- At $20 \%$ of sustained loading, PC specimens without fibers showed one-third the creep coefficient of ABS specimens (0.50 vs. 1.81). With fiber addition, creep coefficient in PC was near one half the creep coefficient in ABS specimens at $20 \%$ loading, i.e., (0.36 vs. 0.83$)$.

- Sustained load level on ABS specimens is suggested to be limited to $20 \%$. At $50 \%$ of sustained loading, PC specimens showed better capacity to carry sustained load than ABS specimens, either with or without fibers.

- Trends observed for the creep tests can be suitably used for knock-down factors. More tests with different fibers, sizing chemistry and stress levels are necessary for suggesting definitive creep coefficients. 


\section{CHAPTER 8}

\section{SUMMARY, CONCLUSIONS AND RECOMMENDATIONS FOR GUARDRAIL POST, RAIL AND OFFSET BLOCK}

\subsection{Introduction}

As a result of this research, an FRP offset block made of recycled polymers and discarded rubber tire strips for joining posts and rails in highway guardrail systems was developed. FRP channel, trapezoidal and box shapes made of recycled resins were tested under tension, bending and compression. A prototype rubber-wood offset block was developed and tested in compression. Issues such as strength of wood for offset blocks and bond stress between rubber and ABS were also evaluated. In the following sections, summary of test results, conclusions and recommendation are given.

\subsection{Summary of Offset Block Manufacturing}

- Three different types of small size offset blocks were manufactured before producing prototype specimens:

- Solid recycled ABS

- Rubber and wood filled recycled ABS

- Two stage molded rubber or wood filled recycled ABS Optimum combination of molding time and molding pressure was established through the manufacturing of small size solid blocks. For the best homogeneity and finish of the molded product, it was established that a 4 minute molding time and 20 Ton molding pressure is required.

Wrapping glass fabric as well as rubber and wood core inclusion was carried out during the manufacturing of small size filled blocks. Based on these 
blocks, further research on bonding of $\mathrm{ABS}$ and rubber was conducted. Manufacturing difficulties such as proper packing of the required amount of pellets and filling the inner zone of the wrap were successfully sorted out during the development of small size filled blocks.

During two stage molding of coupons, it was found that heat transfer through the resin was not effective enough to melt the innermost portion of the specimens. This aspect led to the development of pre-heating concept, which resulted in finalizing a successful molding process.

- Temperature measurements on the mold during molding process showed good heat transfer through the top, bottom and side plates of the mold. However, heat transfer problem was noted based on poor melting of the pellets inside the wrap. Thus, heat transfer through the resin was inadequate to melt the inner pellets within an optimum molding time. Hence, pre-heating of mold and resin prior to compression molding was used as a successful solution to the problem.

- Pre-heated coupons with pre-molded inner tabs (tabs inside the wrap) were compression molded with satisfactory results leading to the manufacture of offset block module.

- A compression molding process was developed to obtain successful prototype offset block modules. The final process includes the following features:

- Rubber core made of discarded automobile tires, which provides good shock absorption to the offset block module. 
- Pre-molding of inner tabs to adequately fill the space and voids between rubber tire core and wrap.

- Pre-heating of mold and ABS pellets to facilitate the melting process during compression molding.

\subsection{Summary of Recycled Polypropylene Channel Section}

- Maximum bending stress of polypropylene (PP) channel section specimens was 13,831 psi. Considering that the specimens were very thin and failed at a low compressive strain, this premature failure can be attributed to combination of compression and punching at the loading point.

- Bending stiffness observed on the channel specimens was $2.45 \times 10^{6}$ psi. for compressive stress and $2.55 \times 10^{6}$ for tensile stress.

- Pure tension properties were not evaluated for polypropylene channel section, due to the similarity of the composite with the trapezoidal section material. Values obtained for trapezoidal polypropylene sections are also valid for channel section.

\subsection{Summary of Recycled Polypropylene Trapezoidal Section}

\section{Bending}

- Maximum bending stresses of polypropylene (PP) trapezoidal section specimens were 45,049 psi for tensile stress and 23,722 psi for compressive stress. Failure was due to compression and punching at the loading point. Tension strength of coupons showed a higher stress value of 72,000 psi to 82,000 psi. 
- Bending stiffness observed on the trapezoidal specimens was $3.04 \times 10^{6}$ psi for compressive stress and $3.16 \times 10^{6}$ psi for tensile stresses.

$\underline{\text { Tension }}$

- Maximum tensile stress of strips from PP trapezoidal section specimens was 82,897 psi.

- Tensile stiffness observed on the strips from PP trapezoidal specimens was $3.83 \times 10^{6} \mathrm{psi}$

Table 8.1 summarizes the mechanical properties established.

Table 8.1 Mechanical Properties of Recycled Trapezoidal Section

\begin{tabular}{|c|c|c|}
\hline Type of test & Stiffness, E (psi) & Ultimate Stress (psi) \\
\hline \multirow{2}{*}{ Bending } & $3.16 \times 10^{6}$ (tensile) & 45,059 (tensile) \\
\cline { 2 - 3 } & $3.04 \times 10^{6}($ compressive) & 23,722 (compressive) \\
\hline Tension & $3.83 \times 10^{6}$ & 82,897 \\
\hline
\end{tabular}

\subsection{Summary of Recycled and Virgin ABS Box Sections}

Bending

- For three point bending tests, maximum bending stress of non-wrapped recycled ABS box section specimens was 12,845 psi. Maximum load under loading point was $280 \mathrm{lb}$.

- For three point bending tests, maximum bending stress of wrapped ABS box section specimens was 12,953 psi. Maximum load under loading point was $504 \mathrm{lb}$. 
- For four point bending tests, maximum bending stress of wrapped ABS box section specimens was 19,733 psi. Maximum load at the point of failure was $589 \mathrm{lb}$.

- Ultimate stress increased from 12,953 psi in 3-point bending to 19,733 psi in 4-point bending. However, applied load at loading points just increased from $504 \mathrm{lbs}$. to $589 \mathrm{lbs}$. This fact indicates that for the wrapped recycled ABS box sections a punching failure occurs between 504 to $589 \mathrm{lbs}$. The difference between the two punching loads is possibly due to the non-uniform section thickness.

- Ultimate tensile bending stress for virgin $\mathrm{ABS}$ box sections failing in compression was 29,972 psi.

- Tensile bending stiffness for virgin ABS box sections was $3.60 \times 10^{6}$ psi whereas compressive bending stiffness was $2.52 \times 10^{6} \mathrm{psi}$.

$\underline{\text { Tension }}$

- Maximum tensile stress of recycled ABS box section was 19,960 psi.

- Tensile stiffness of recycled ABS box section was $3.21 \times 10^{6} \mathrm{psi}$.

Mechanical properties of box sections are summarized in Table 8.2 
Table 8.2 Mechanical Properties of Recycled and Virgin Box Sections

\begin{tabular}{|c|c|c|c|}
\hline $\begin{array}{c}\text { Type of ABS box } \\
\text { section }\end{array}$ & $\begin{array}{c}\text { Mechanical } \\
\text { Property }\end{array}$ & Stiffness, E (psi) & $\begin{array}{c}\text { Ultimate Stress } \\
\text { (psi) }\end{array}$ \\
\hline \multirow{2}{*}{ Recycled } & Bending & $3.86 \times 10^{6}$ (tensile) & \multirow{2}{*}{12,845} \\
\cline { 3 - 3 } & Tension & $3.28 \times 10^{6}($ compressive) & \multirow{2}{*}{$3.21 \times 10^{6}$} \\
\cline { 3 - 3 } Recycled (wrapped) & Bending & $3.42 \times 10^{6}$ (tensile) & \multirow{2}{*}{19,733} \\
\cline { 3 - 3 } & & $2.94 \times 10^{6}($ compressive) & \multirow{2}{*}{29,972} \\
\hline \multirow{2}{*}{ Virgin } & Bending & $3.60 \times 10^{6}$ (tensile) & \multirow{2}{*}{$2.52 \times 10^{6}$ (tensile) } \\
\cline { 3 - 3 } & &
\end{tabular}

\subsection{Summary of Recycled ABS Sheets}

$\underline{\text { Bending }}$

- Maximum bending stress of recycled ABS sheets for short plates was 27,991 psi. A shear failure causing a delamination of the plate was noted at a stress of 874 psi.

- Bending stiffness of recycled ABS short plates was $2.59 \times 10^{6}$ psi for tensile bending stress and $2.33 \times 10^{6}$ psi for compressive bending stress.

- Long sheet recycled ABS specimens had an ultimate bending stress of 39,518 psi. Compression failure (crushing) was observed. Shear stress between laminates at this bending stress level was 400 psi.

- Bending stiffness of recycled ABS sheets was $3.03 \times 10^{6}$ psi for tensile bending stress and $2.91 \times 10^{6}$ psi for compressive bending stress. 


\section{$\underline{\text { Tension }}$}

- Maximum tensile stress of recycled ABS sheets was 21,950 psi.

- Tensile stiffness of recycled ABS sheets was $2.68 \times 10^{6} \mathrm{psi}$.

\section{$\underline{\text { Compression }}$}

- Maximum compressive stress of strips from ABS sheet specimens was 6,900 psi. Specimens delaminated instead of crushing due to its large aspect ratio.

- Compressive stiffness for recycled ABS sheets was $3.58 \times 10^{6} \mathrm{psi}$.

Mechanical properties of recycled ABS sheets are summarized in Table 8.3

Table 8.3 Mechanical Properties of Recycled ABS Sheets

\begin{tabular}{|c|c|c|}
\hline Mechanical property & Stiffness, E (psi) & Ultimate Stress (psi) \\
\hline \multirow{2}{*}{ Bending } & $3.03 \times 10^{6}$ (tensile) & 39,518 \\
\cline { 2 - 3 } & $2.91 \times 10^{6}$ (compressive) & \\
\hline Shear (between laminates) & - & 874 \\
\hline Tension & $2.68 \times 10^{6}$ & 21,950 \\
\hline Compression & $3.58 \times 10^{6}$ & $\begin{array}{c}6,900 \text { (premature failure } \\
\text { due to delamination) }\end{array}$ \\
\hline
\end{tabular}

\subsection{Summary of Recycled ABS Belt-Type Material}

- Maximum tensile stress of strips from ABS belt-type specimens were 29,800 for type A, 37,150 psi for type B and 24,450 for type C. Fiber volume fractions for each type of specimens were: $20 \%$ for A, $24 \%$ for B and $19 \%$ for specimen type $\mathrm{C}$.

- Tensile stiffness observed on the strips from ABS belt-type specimens was $2.92 \times 10^{6}$ psi for type A, $2.65 \times 10^{6}$ psi for type B, and $2.09 \times 10^{6}$ psi for type C. 


\subsection{Summary of Rubber-Wood Offset Block Model}

Performance of the block was considered satisfactory, holding together during the compression test. Buckling of rubber strips at 5,250 lbs was expected as they were not laterally restrained. No damage was noted in the wood or rubber parts.

\subsection{Summary of Tests on Wood Used in Rubber - Wood Model}

\section{Compression}

- Maximum compressive stress parallel to grain of wood was found to be 8,399 psi. Maximum compressive stress perpendicular to grain was 1,472 psi.

- Compressive stiffness parallel to grain was $1.63 \times 10^{6}$ psi while compressive stiffness perpendicular to grain was $0.197 \times 10^{6}$ psi.

$\underline{\text { Impact }}$

- Izod impact strength was $1.82 \mathrm{ft}-\mathrm{lb} / \mathrm{in}$. This value is very low compared to non- reinforced and virgin polymers.

Mechanical properties of recycled ABS sheets are summarized in Table 8.4

Table 8.4 Mechanical Properties of Wood from Rubber-Wood Model

\begin{tabular}{|c|c|c|}
\hline Mechanical property & Stiffness, E (psi) & Ultimate Stress (psi) \\
\hline Compression parallel to grain & $1.63 \times 10^{6}$ & 8,399 \\
\hline Compression perpendicular to grain & $0.197 \times 10^{6}$ & 1,472 \\
\hline Impact Strength (Izod) & - & $1.82^{*}$ \\
\hline
\end{tabular}

* Impact strength is in ft-lb/in

\subsection{Summary of Bond Strength on Rubber-ABS Interface}

- Primer application on rubber surface increased the bond strength between 2.44 to 3.76 times. 
- Specimens cured during 8 minutes showed increase 2.04 times in bond strength as compared to specimens cured for 4 minutes. $7 \%$ gain in bond strength (1.07 times) was observed for specimens cured for 8 minutes as compared to specimens cured for 6 minutes.

- Remolding of specimens (secondary curing) increased the bond strength between 1.11 to 1.72 times.

\subsection{Conclusions and Recommendations}

\section{$\underline{\text { Offset block manufacturing }}$}

- For a molding temperature of $400^{\circ} \mathrm{F}$, optimum molding time and molding pressure were established by trying different combinations. For a 3 "x 3 "x $0.5 "$ ABS product 4 minute molding gave best results. However, for larger samples optimum molding time is higher. For the final offset block module measuring $14 " \mathrm{x} 8$ "x2" an optimum molding time of 10 minutes was determined.

- Heating the specimens for more than 12 minutes at $400^{\circ} \mathrm{F}$ is not recommended. It was observed throughout this research that samples molded for more than 12 minutes will have a brown finish (burn marks) on top and bottom surfaces. Also, overheated resins will try to expand out of the mold after releasing the molding pressure, thus lifting up the top plate and leading to a product with rough finish and voids.

- It was observed that the smaller the size of the pellets, the easier it is to fill the voids in the mold. However, this may be a disadvantage in terms of handling the specimen before preheating, because keeping the pellet pile in place becomes more difficult. No improvement on the final product finish or 
homogeneity is expected from using small (ground) pellets if adequate melting and cure time are utilized.

- In order to overcome the heat transfer problems through the resin, an alternate approach to pre-heating of the pellets is the use of smaller pellets, this would facilitate the heat transfer through the resin. A minimum effective pellet size has to be determined by trial. Other approach would be blanketing the polymer with an inert gas. This would cool down the outside pellets to prevent degradation from overheating while the heat is transferred to the inner pellets in the mold. Installation of thermocouples inside the specimens and thermography procedures will help in understanding the heat transfer through the polymer molded samples.

- Discarded rubber tire strips used in this research were manually cut with a reciprocating saw. Cut edges obtained were non-uniform and posed placement problems for lateral pre-molded tabs. In a mass production process, it is recommended to improve the uniformity of rubber tire edges by means of a different cutting mechanism such as shearing or heating. Use of uniform rubber cores will also lead to the use of uniform ABS pre-cast tabs.

- The compression molding machine (12.5" x 12.5") available at WVU-CFC was not large enough to conduct compression molding of a module in a single heating stage. A compression molding machine with hot platens at least $15 \mathrm{"} \mathrm{x}$ $10 "$ must be used to avoid additional compression molding of the portion left out of the plates. 
- Use of aluminum flashing as a cover for mold plates gave smoother finish than aluminum foil that gave a wrinkly finish. In addition, ease of de-molding was observed when aluminum flashing was used.

- Caution must be used at every stage of the process due to the manufacturing of large and heavy mold blocks at high temperatures. High temperature resistance gloves must be worn when handling hot elements. Special attention must be taken when carrying the pre-heated specimen from the oven to the molding machine. Pre-heated parts at $400^{\circ} \mathrm{F}$ (bottom plate + mold + pellets) weigh around 25 pounds. Use of a cart is recommended for moving these heavy mold and block assembly.

- One of the difficulties in the specimen preparation was to adequately fill the gap between rubber core and fabric wrap with ABS pellets. Pre-casting ABS tabs, which constitutes an additional step to be carried out before compression molding of the modules solved this problem. Molds that allow air escape from the specimens while molding would also contribute to void reduction.

- Alternate molding methods can be considered to overcome manufacturing difficulties. Injection molding process may demand less manufacturing effort. However, a proper injection mold capable of holding a core and a fiber wrap has to be developed.

- A successful procedure for compression molding of offset block was developed. Efforts need to be concentrated in characterizing the mechanical properties (tension, compression, bending and impact) of the modules and the offset block as a whole through standard but expensive crash tests. 
- A new use for discarded rubber tires was found taking advantage of its shock absorption properties. Discarded rubber tires rubber is heavier than ABS resin. Weight of the finished module was around $7 \mathrm{lbs}$. Other core materials such as sawdust can also be evaluated to obtain a lighter final product.

- It is recommended that further optimization and improvement of the offset block can be carried out through finite element modeling.

\section{Post and Rail}

- Recycled Polypropylene channel and trapezoidal sections as well as recycled ABS box sections were tested. The three shapes, when tested in bending exhibited a combination of compression and punching failure. This failure mode is mostly due to small section thickness of $0.10^{\prime \prime}$ to $0.16 "$. Testing of actual size specimens will give a better knowledge of punching behavior. Also, additional reinforcement at loading points (joints) can be implemented in actual guardrail systems to improve the load transfer and effectiveness of the sections.

- Stress and strain values obtained from the tests indicate suitability of all the shapes for use in highway guardrail systems. Recycled polypropylene shapes (channel and trapezoidal) showed better performance than recycled ABS box shape. However PP specimens had a fiber volume fraction of 41 to $43 \%$, while ABS samples had 25\%. Trapezoidal shape was the most effective section in terms of bending resistance. 
- Tensile strength of recycled PP specimens was found to be 82,897 psi. This indicates that if punching problems are avoided, the material strength can be utilized more effectively.

- From the available sections of different shapes, it was difficult to obtain representative compression specimens due to the small thickness. Compression strength can be determined from specimens cut from actual size shapes for guardrails.

- Recycled ABS box section showed lower bending resistance as compared to other shapes tested. However, it was noted that these sections had a nonuniform thickness from 0.05 " to $0.15 "$. Improvements in the manufacturing process will lead to more uniform section with better mechanical properties.

- Wrapping of recycled ABS box sections contributed to an increase in the ultimate strength and punching resistance of the section.

- Recycled ABS sheets having a fiber volume fraction of $25 \%$ performed well under bending. Failure mode observed was crushing of the compression surface. Thickness of $0.375^{\prime \prime}$ for these sheets prevented a punching failure, which was observed for box sections with 0.10 " thickness. Bending of short plates led to interlaminar shear failure at a maximum shear stress of $874 \mathrm{psi}$. Slender specimens in compression also failed in shear. It is recommended to improve the wet-out of fibers during manufacturing process to increase the shear strength.

- Recycled ABS belt-type specimens (tension elements) showed tensile resistance of 24,450 to 37,150 psi. Based on these results, it was concluded 
that belt-type elements are suitable for carrying tensile loads on structural systems. However, due to their reduced thickness, they might be very sensitive to external agents such as lateral impact forces or harsh environment. Use of protective coating can alleviate this anticipated problem.

- A rubber-wood offset block prototype with no lateral restraint was manufactured and tested in compression. The need for lateral restraint to prevent buckling and a change in rubber-wood block considerations was evident from the tests.

- Wood from rubber - wood prototype was tested in compression parallel and perpendicular to grain. Compressive stress parallel to grain of 8,399 psi was comparable to virgin and recycled ABS compressive stress of 8,792 psi to 8,972 psi reported by (Bargo,2000). However, wood compressive strength was less than compressive stress for virgin and recycled PC (10,311 psi to $10,523 \mathrm{psi})$.

- Compressive stress of wood (8,399 psi) was also found to be less than compressive strength of reinforced polymers. Values established by (Bargo, 2000) are 13,135 psi to 14,891 psi for virgin and recycled ABS and 20,332 psi to 21,124 psi for virgin and recycled PC.

- Impact strength of wood for offset block was less than virgin ABS and PC impact resistance. However, impact strength of wood is comparable to that of recycled ABS and higher than fiber reinforced ABS. 
- Impact strength of all combinations of virgin and recycled PC with and without fiber addition evaluated by (Bargo,2000), showed higher values than those obtained for wood specimens from offset blocks.

- It was determined that better bond strength between ABS and rubber was obtained when primer was applied to the bonding surface. Curing time of 8 minute also showed better results than 6 and 4 minute curing. Remolding of specimens resulted in a gain of $10 \%$ in bond strength.

- Based on test results and manufacturing issues, it is concluded that recycled polymers are suitable for developing highway and automotive applications.

$\underline{\text { Recommendation for Future Research: }}$

Further research should be carried out on:

- Use of continuous fiber / fabrics for product manufacturing

- Development of a more efficient offset block in terms of lighter weight

- Crash testing of manufactured offset blocks

- Improvement of recycled polymer shapes in terms of fiber / fabric architecture and manufacturing

- Testing of actual size polymer shapes

- Developing design philosophies for recycled polymer posts, rails and offset blocks for highway guardrail systems 


\section{REFERENCES}

Ackerman, F. (1997). Why do we recycle? Markets, values, and public policy.

Washington, DC: Island Press.

Agarwal, B. D., Broutman, L. J. (1980). Analysis and Performance of Fiber Composites. New York, NY: John Wiley \& Sons.

Bargo, J. E. (2000). Mechanical property characterization of recycled thermoplastics. CFC Report No. 00-287 and Thesis Report, Constructed Facilities Center, West Virginia University, West Virginia.

Barton, A. F. M. (1979). Resource Recovery and Recycling. New York: WileyInterscience.

Beer, F. P., \& Johnston, E. R. (1991). Mecánica de materiales. Bogotá, Colombia: McGraw-Hill Latinoamericana.

Bennett, R. A. (1992). Recycled plastics. Product applications and potential. In Gerald D. Andrews and Pallatheri M. Subramanian (Ed.), ACS Symposium Series: Vol. 513. Emerging Technologies in Plastic Recycling (pp. 26-38). Washington, DC: American Chemical Society.

BLI Series Operation Manual for Impact Testing (1999).

Durotronic Model 2000 Operating Manual (1999).

Dutta, P. K. (1998). Investigations of plastic composite materials for highway safety structures (CRREL Rep. No. 98-7). Hanover, NH: US Army Corps Engineers.

GangaRao, H. V. S., Vijay P.V., Dutta P. K. (1995). Durability of Composites in Infrastructure. The NACE International Conference and Corrosion Show, Paper No. 550 . 
http://www.plasticresource.com/recycling/recycling-backgronder/plastic lumber.html

http://www.rivrdeep.net/current/2000/04/front.280400.recycle.jhtml

http://uc.rutgers.edu/news/science/plastic-railroad2.html

Instron Series 8500 Two-Column Load Frame Operating Manual (1999).

McLaren, M. G., Assis G., Penisero J., McClaren, M. G., Melewski, P. M., $\quad$ Lashway, K. F., Krishnaswamy, P. (2002). Introducing the first recycled plastic bridge in the world. $I B C, 01-65$.

Modern Plastics. (Jan. 2000). Partnership helps injection molder penetrate new market segments, p. 32-33.

Nilson, A. H., Winter, G. (1986). Design of Concrete Structures. Mc-Graw-Hill Book Co, Singapore

Powelson, D., Powelson, M. (1992). The recycler's manual for business, government, and the environmental community. Van Nostrand Reinhold, New York.

Springer, G. S. (1981). Environmental Effects on Composite Materials. Technomac Publishing Company Inc. (Vol. I, II, III).

Tall, S., Karlsson, S., Albertson, A. (1998). Improvements in the properties of mechanically recycled thermoplastics. Polymer \& Polymer Composites, Vol. 6, No. 5 .

Vijay, P.V., GangaRao H.V.S. (1999). Accelerated and Natural Weathering of Glass Fiber Reinforced Plastic Bars, Fiber Reinforced Polymer Reinforcement for Reinforced Concrete Structures, Fourth International Symposium (pp. 605-613). Farmington Hills, Michigan: American Concrete Institute. 


\section{APPENDIX A}

TENSION TEST RESULTS FOR CONDITIONED SPECIMENS

Table A.1 Tension Test Results for 2 Months of Aging

\begin{tabular}{|c|c|c|c|c|c|c|c|c|}
\hline Type & Specimen & $\begin{array}{l}\text { Maximum } \\
\text { Load } \\
\text { lbs. }\end{array}$ & \begin{tabular}{|c} 
Maximum \\
Stress \\
psi
\end{tabular} & $\begin{array}{c}\text { Average } \\
\text { Max. Stress } \\
\text { psi }\end{array}$ & \begin{tabular}{|c|} 
Strain @ \\
Max. load \\
$\%$
\end{tabular} & $\begin{array}{c}\text { Max. Strain } \\
@ \text { Failure } \\
\%\end{array}$ & $\begin{array}{c}\text { Stiffness, E } \\
\text { psi } \times 10^{6}\end{array}$ & $\begin{array}{c}\text { Average } \\
\text { Stiffness, } \\
\text { psi } \times 10^{6}\end{array}$ \\
\hline \multirow{6}{*}{ ABS } & TA1-9 & 341 & 5866 & 5866 & \multicolumn{4}{|c|}{ No strains were recorded at 2 months of aging } \\
\hline & TA2-12 & 582 & 9683 & 9683 & & & & \\
\hline & TA3-11 & 308 & 5285 & 5285 & & & & \\
\hline & TA4-14 & 513 & 8802 & 5285 & & & & \\
\hline & TA5-10 & 325 & 5558 & 5558 & & & & \\
\hline & TA6-12 & 561 & 9607 & 9607 & & & & \\
\hline \multirow{6}{*}{ PC } & TP1-6 & 510 & 8210 & 8210 & & & & \\
\hline & TP2-7 & 959 & 15101 & 15101 & & & & \\
\hline & TP3-6 & 504 & 7813 & 7813 & & & & \\
\hline & TP4-6 & 893 & 14203 & 14203 & & & & \\
\hline & TP5-7 & 532 & 8072 & 8072 & & & & \\
\hline & TP6-6 & 964 & 14831 & 14831 & & & & \\
\hline
\end{tabular}

Table A.2 Tension Test Results for 4 Months of Aging

\begin{tabular}{|c|c|c|c|c|c|c|c|c|}
\hline \begin{tabular}{|l|} 
Type \\
\end{tabular} & Specimen & $\begin{array}{l}\text { Maximum } \\
\text { Load } \\
\text { lbs. }\end{array}$ & $\begin{array}{c}\text { Maximum } \\
\text { Stress } \\
\text { psi }\end{array}$ & $\begin{array}{c}\text { Average } \\
\text { Max. Stress } \\
\text { psi }\end{array}$ & \begin{tabular}{|c} 
Strain @ \\
Max. load \\
$\%$
\end{tabular} & \begin{tabular}{|c|} 
Max. Strain \\
@ Failure \\
$\%$
\end{tabular} & $\begin{array}{c}\text { Stiffness, E } \\
\text { psi } \times 10^{6}\end{array}$ & $\begin{array}{c}\text { Average } \\
\text { Stiffness, E } \\
\text { psi } \times 10^{6}\end{array}$ \\
\hline \multirow{6}{*}{ ABS } & TA1-10 & - & - & - & - & - & - & - \\
\hline & TA2-13 & 606 & 10510 & 10510 & - & - & 1.019 & 1.019 \\
\hline & TA3-13 & 337 & 5988 & 5988 & 0.96 & 5.08 & - & - \\
\hline & TA4-15 & 550 & 9388 & 9388 & 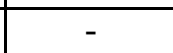 & 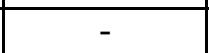 & - & - \\
\hline & TA5-11 & - & - & - & 0.99 & 1.08 & 0.360 & 0.360 \\
\hline & TA6-13 & 608 & 10619 & 10619 & - & - & 1.050 & 1.050 \\
\hline \multirow{5}{*}{ PC } & TP1-10 & 578 & 9261 & 9261 & - & 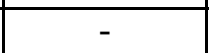 & 0.309 & 0.309 \\
\hline & TP2-8 & 994 & 15721 & 15721 & - & - & 1.008 & 1.008 \\
\hline & TP3-8 & 570 & 9233 & 9233 & - & - & 0.315 & 0.315 \\
\hline & TP4-9 & 891 & 14205 & 14205 & - & - & 0.964 & 0.964 \\
\hline & TP5-8 & 606 & 9163 & 9163 & - & - & 0.337 & 0.337 \\
\hline
\end{tabular}


Table A.3 Tension Test Results for 10 Months of Aging

\begin{tabular}{|c|c|c|c|c|c|c|c|c|}
\hline Type & Specimen & $\begin{array}{l}\text { Maximum } \\
\text { Load } \\
\text { lbs. }\end{array}$ & $\begin{array}{c}\text { Maximum } \\
\text { Stress } \\
\text { psi }\end{array}$ & $\begin{array}{c}\text { Average } \\
\text { Max. Stress } \\
\text { psi }\end{array}$ & $\begin{array}{c}\text { Strain @ } \\
\text { Max. load } \\
\%\end{array}$ & $\begin{array}{c}\text { Max. Strain } \\
\text { @ Failure } \\
\%\end{array}$ & $\begin{array}{c}\text { Stiffness, E } \\
\text { psi } \times 10^{6}\end{array}$ & $\begin{array}{c}\text { Average } \\
\text { Stiffness, E } \\
\text { psi } \times 10^{6}\end{array}$ \\
\hline \multirow{11}{*}{$A B S$} & TA1-12 & 348 & 6118 & 6118 & 2.02 & 2.12 & 0.360 & 0.360 \\
\hline & TA2-14 & 506 & 9084 & & 0.97 & 1.70 & 1.005 & \\
\hline & TA2-15 & 542 & 9736 & 9410 & 1.09 & 1.09 & 1.089 & 1.047 \\
\hline & TA3-16 & 317 & 5580 & \multirow[b]{2}{*}{5549} & 2.07 & 2.71 & 0.336 & \multirow[b]{2}{*}{0.337} \\
\hline & TA3-17 & 320 & 5518 & & 2.39 & 8.18 & 0.338 & \\
\hline & TA4-16 & 417 & 7346 & \multirow[b]{2}{*}{7602} & 0.85 & 0.85 & 0.969 & \multirow[b]{2}{*}{0.909} \\
\hline & TA4-17 & 447 & 7858 & & 0.60 & 0.60 & 0.849 & \\
\hline & TA5-12 & 313 & 5693 & & 1.79 & 1.79 & 0.343 & \multirow[b]{2}{*}{0.347} \\
\hline & TA5-13 & 325 & 5888 & 5790 & 1.97 & 1.98 & 0.351 & \\
\hline & TA6-15 & - & - & - & - & - & 1.019 & \\
\hline & TA6-16 & 518 & 9124 & 9124 & 1.04 & 1.04 & 0.967 & 0.993 \\
\hline \multirow{11}{*}{$\mathrm{PC}$} & TP1-10 & 541 & 8817 & 8817 & 6.88 & 13.56 & 0.364 & 0.364 \\
\hline & TP2-12 & 812 & 12975 & & 1.79 & 1.79 & 1.072 & \multirow[b]{2}{*}{1.076} \\
\hline & TP2-15 & 802 & 12758 & 12867 & 1.74 & 1.74 & 1.080 & \\
\hline & TP3-10 & 539 & 8978 & \multirow[b]{2}{*}{8983} & 6.56 & 13.62 & 0.374 & \multirow[b]{2}{*}{0.364} \\
\hline & TP3-11 & 540 & 8989 & & 6.74 & 13.57 & 0.353 & \\
\hline & TP4-10 & 751 & 12454 & \multirow[b]{2}{*}{12525} & 1.60 & 1.60 & 1.104 & \multirow[b]{2}{*}{1.061} \\
\hline & TP4-11 & 772 & 12596 & & 1.68 & 1.68 & 1.018 & \\
\hline & TP5-10 & 521 & 8160 & \multirow[b]{2}{*}{8589} & 3.57 & 3.58 & 0.343 & \multirow[b]{2}{*}{0.347} \\
\hline & TP5-11 & 580 & 9019 & & 7.07 & 13.53 & 0.350 & \\
\hline & TP6-12 & 786 & 12619 & \multirow[b]{2}{*}{12877} & 1.57 & 1.57 & 1.098 & \multirow[b]{2}{*}{1.099} \\
\hline & TP6-16 & 822 & 13135 & & 1.65 & 1.84 & 1.099 & \\
\hline
\end{tabular}


Table A.4 Tension Test Results for 18 Months of Aging

\begin{tabular}{|c|c|c|c|c|c|c|c|c|}
\hline Type & Specimen & $\begin{array}{l}\text { Maximum } \\
\text { Load } \\
\text { lbs. }\end{array}$ & $\begin{array}{l}\text { Maximum } \\
\text { Stress } \\
\text { psi }\end{array}$ & \begin{tabular}{|c} 
Average \\
Max. Stress \\
psi
\end{tabular} & $\begin{array}{c}\text { Strain @ } \\
\text { Max. load } \\
\%\end{array}$ & $\begin{array}{c}\text { Max. Strain } \\
\text { @ Failure } \\
\%\end{array}$ & $\begin{array}{l}\text { Stiffness, E } \\
\text { psi x } 10^{6}\end{array}$ & $\begin{array}{c}\text { Average } \\
\text { Stiffness, E } \\
\text { psi } \times 10^{6}\end{array}$ \\
\hline \multirow{12}{*}{$A B S$} & TA1-13 & 336 & 5728 & & 1.97 & 2.83 & 0.348 & \\
\hline & TA1-14 & 337 & 5890 & 5809 & 1.90 & 2.77 & 0.375 & 0.362 \\
\hline & TA2-16 & 593 & 10439 & & 1.23 & 1.23 & 1.010 & \\
\hline & TA2-17 & 614 & 10917 & 10678 & 1.27 & 1.28 & 1.043 & 1.027 \\
\hline & TA3-19 & 311 & 5319 & & 1.93 & 4.10 & 0.334 & \\
\hline & TA3-20 & 312 & 5402 & 5360 & 1.96 & 4.14 & 0.328 & 0.331 \\
\hline & TA4-18 & 498 & 8516 & & 1.02 & 1.04 & 1.001 & \\
\hline & TA4-19 & 493 & 8759 & 8637 & 1.00 & 1.59 & 1.022 & 1.012 \\
\hline & TA5-14 & 332 & 5838 & & 1.93 & 3.56 & 0.348 & \\
\hline & TA5-15 & 316 & 5528 & 5683 & 1.81 & 1.87 & 0.353 & 0.351 \\
\hline & TA6-16 & 524 & 9000 & & 1.07 & 1.07 & 1.029 & \\
\hline & TA6-17 & 210 & 8832 & 8916 & 1.04 & 1.04 & 1.053 & 1.041 \\
\hline \multirow{12}{*}{$\mathrm{PC}$} & TP1-18 & 527 & 8320 & & 5.17 & 9.12 & 0.349 & \\
\hline & TP1-21 & 521 & 8383 & 8352 & 5.34 & 9.21 & 0.350 & 0.35 \\
\hline & TP2-16 & 749 & 11931 & & 1.65 & 1.65 & 1.029 & \\
\hline & TP2-20 & 744 & 11853 & 11892 & 1.61 & 1.61 & 1.107 & 1.068 \\
\hline & TP3-12 & 515 & 8352 & & 5.46 & 8.90 & 0.344 & \\
\hline & TP3-13 & 512 & 8307 & 8329 & 5.36 & 9.40 & 0.341 & 0.343 \\
\hline & TP4-16 & 665 & 10498 & & 1.43 & 1.43 & 1.050 & \\
\hline & TP4-21 & 719 & 11343 & 10920 & 1.71 & 1.71 & 1.095 & 1.073 \\
\hline & TP5-12 & 538 & 8239 & & 5.11 & 9.79 & 0.345 & \\
\hline & TP5-17 & 548 & 8132 & 8185 & 5.12 & 9.13 & 0.352 & 0.349 \\
\hline & TP6-17 & 753 & 11855 & & 1.67 & 1.68 & 1.094 & \\
\hline & TP6-21 & 753 & 11842 & 11848 & 1.65 & 1.65 & 1.087 & 1.091 \\
\hline
\end{tabular}




\section{APPENDIX B}

\section{BENDING TEST RESULTS FOR CONDITIONED SPECIMENS}

Table B.1 Bending Test Results for 2 Months of Aging

\begin{tabular}{|c|c|c|c|c|c|c|}
\hline Type & Specimen & $\begin{array}{c}\text { Maximum } \\
\text { Load } \\
\text { Ibs. }\end{array}$ & $\begin{array}{c}\text { Maximum } \\
\text { Stress } \\
\text { psi }\end{array}$ & $\begin{array}{c}\text { Deflection at } \\
\text { Max. Stress } \\
\text { in }\end{array}$ & $\begin{array}{c}\text { Max. Deflection } \\
\text { Recorded } \\
\text { in }\end{array}$ & $\begin{array}{c}\text { Stiffness, E } \\
\text { Psi x 10 }\end{array}$ \\
\hline \multirow{4}{*}{ ABS } & BA2-18 & 25.2 & 16033 & 0.41 & 0.43 & - \\
\cline { 2 - 7 } & BA3-19 & 13.5 & 8931 & 0.49 & 0.49 & - \\
\cline { 2 - 7 } & BA4-18 & 23.2 & 15522 & 0.33 & 0.37 & - \\
\cline { 2 - 7 } & BA5-18 & 13.7 & 9295 & 0.49 & 0.49 & - \\
\cline { 2 - 7 } & BA6-19 & 26.1 & 17793 & 0.41 & 0.45 & - \\
\hline \multirow{5}{*}{ PC } & BP1-11 & 11.1 & 7494 & 0.43 & 0.45 & - \\
\cline { 2 - 7 } & BP2-12 & 30.6 & 20228 & 0.42 & 0.47 & - \\
\cline { 2 - 7 } & BP3-11 & 11.8 & 8235 & 0.45 & 0.46 & - \\
\cline { 2 - 7 } & BP4-12 & 27.1 & 18830 & 0.45 & 0.46 & - \\
\cline { 2 - 7 } & BP5-11 & 16.2 & 10136 & 0.44 & 0.45 & - \\
\cline { 2 - 7 } & BP6-11 & 29.6 & 19606 & 0.44 & 0.45 & - \\
\hline
\end{tabular}

Table B.2 Bending Test Results for 4 Months of Aging

\begin{tabular}{|c|c|c|c|c|c|c|}
\hline Type & Specimen & \begin{tabular}{|c} 
Maximum \\
Load \\
lbs.
\end{tabular} & \begin{tabular}{|c|} 
Maximum \\
Stress \\
psi
\end{tabular} & \begin{tabular}{|c|} 
Deflection at \\
Max. Stress \\
in
\end{tabular} & \begin{tabular}{|c|}
$\begin{array}{c}\text { Max. Deflection } \\
\text { Recorded } \\
\text { in }\end{array}$ \\
\end{tabular} & $\begin{array}{r}\text { Stiffness, E } \\
\text { psi x } 10^{6} \\
\end{array}$ \\
\hline \multirow{6}{*}{ ABS } & BA1-5 & 35.2 & 6728 & 0.30 & 0.37 & 0.334 \\
\hline & BA2-19 & 36.2 & 15211 & 0.38 & 0.39 & 0.932 \\
\hline & BA3-20 & 11.4 & 7862 & 0.50 & 0.80 & 0.364 \\
\hline & BA4-19 & 19.2 & 13886 & 0.31 & 0.33 & 0.93 \\
\hline & BA5-19 & 12.2 & 8412 & 0.57 & 0.82 & 0.346 \\
\hline & BA6-20 & 20.6 & 14043 & 0.34 & 0.36 & 0.917 \\
\hline \multirow{6}{*}{ PC } & BP1-13 & 13.5 & 9283 & 0.63 & 0.94 & 0.341 \\
\hline & BP2-13 & 29.6 & 21336 & 0.47 & 0.50 & 1.001 \\
\hline & BP3-12 & 11.7 & 8398 & 0.59 & 0.67 & 0.354 \\
\hline & BP4-13 & 23.5 & 17117 & 0.38 & 0.42 & 0.994 \\
\hline & BP5-12 & 14.5 & 10602 & 0.77 & 0.78 & 0.406 \\
\hline & BP6-12 & 28.8 & 21159 & 0.44 & 0.45 & 1.062 \\
\hline
\end{tabular}


Table B.3 Bending Test Results for 10 Months of Aging

\begin{tabular}{|c|c|c|c|c|c|c|c|}
\hline Type & Specimen & \begin{tabular}{|c|} 
Maximum \\
Load \\
lbs.
\end{tabular} & $\begin{array}{l}\text { Maximum } \\
\text { Stress } \\
\text { psi }\end{array}$ & $\begin{array}{c}\text { Average } \\
\text { Max. Stress } \\
\text { psi }\end{array}$ & $\begin{array}{l}\text { Deflection at } \\
\text { Max. Stress } \\
\text { in }\end{array}$ & $\begin{array}{c}\text { Max. Deflection } \\
\text { Recorded } \\
\text { in }\end{array}$ & $\begin{array}{r}\text { Stiffness, E } \\
\text { psi x } 10^{6}\end{array}$ \\
\hline \multirow{12}{*}{$A B S$} & BA1-8 & \multicolumn{2}{|c|}{ Not tested to failure } & & - & - & 0.699 \\
\hline & BA1-9 & 60.1 & 10747 & 10747 & 0.51 & 0.95 & - \\
\hline & BA2-6 & \multicolumn{2}{|c|}{ Not tested to failure } & & - & - & 1.215 \\
\hline & BA2-7 & 82.2 & 14631 & 14631 & 0.22 & 0.22 & - \\
\hline & BA3-6 & \multicolumn{2}{|c|}{ Not tested to failure } & & - & - & 0.408 \\
\hline & BA3-7 & 50.5 & 8923 & 8923 & 0.32 & 0.32 & - \\
\hline & BA4-3 & 64.0 & 11235 & & 0.15 & 0.15 & 0.998 \\
\hline & BA4-6 & 74.5 & 13088 & 12162 & 0.20 & 0.20 & - \\
\hline & BA5-3 & 57.7 & 10107 & & 0.40 & 0.44 & 0.385 \\
\hline & BA5-4 & 61.1 & 10785 & 10446 & 0.50 & 0.79 & - \\
\hline & BA6-3 & 65.1 & 11263 & & 0.19 & 0.19 & 0.925 \\
\hline & BA6-4 & 66.0 & 11537 & 11400 & 0.27 & 0.28 & - \\
\hline \multirow{12}{*}{$P C$} & BP1-14 & 18.4 & 12476 & \multirow[b]{2}{*}{14945} & 0.64 & 0.65 & 0.416 \\
\hline & BP1-16 & 25.4 & 17414 & & 1.08 & 1.23 & - \\
\hline & BP2-14 & 29.7 & 20185 & \multirow[b]{2}{*}{25370} & 0.40 & 0.40 & 1.089 \\
\hline & BP2-15 & 45.9 & 30555 & & 0.48 & 0.48 & - \\
\hline & BP3-13 & 24.4 & 16368 & \multirow[b]{2}{*}{17327} & 0.93 & 1.00 & 0.445 \\
\hline & BP3-14 & 26.1 & 18286 & & 1.22 & 1.24 & - \\
\hline & BP4-16 & 49.1 & 33187 & \multirow[b]{2}{*}{28586} & 0.43 & 0.43 & 1.001 \\
\hline & BP4-17 & 35.5 & 23985 & & 0.48 & 0.48 & - \\
\hline & BP5-13 & 20.6 & 13306 & \multirow[b]{2}{*}{15023} & 0.64 & 0.64 & 0.452 \\
\hline & BP5-14 & 26.9 & 16740 & & 1.24 & 1.24 & - \\
\hline & BP6-13 & 32.8 & 21537 & \multirow[b]{2}{*}{23909} & 0.41 & 0.52 & 1.168 \\
\hline & BP6-15 & 38.9 & 26281 & & 0.45 & 0.47 & - \\
\hline
\end{tabular}


Table B.4 Bending Test Results for 18 Months of Aging

\begin{tabular}{|c|c|c|c|c|c|c|c|}
\hline Type & Specimen & $\begin{array}{c}\text { Maximum } \\
\text { Load } \\
\text { lbs. }\end{array}$ & $\begin{array}{c}\text { Maximum } \\
\text { Stress } \\
\text { psi }\end{array}$ & $\begin{array}{c}\text { Average } \\
\text { Max. Stress } \\
\text { psi }\end{array}$ & $\begin{array}{c}\text { Deflection at } \\
\text { Max. Stress } \\
\text { in }\end{array}$ & $\begin{array}{c}\text { Max. Deflection } \\
\text { Recorded } \\
\text { in }\end{array}$ & Stiffness, E \\
psi x 10
\end{tabular}




\section{APPENDIX C}

\section{COMPRESSION TEST RESULTS FOR CONDITIONED SPECIMENS}

Table C.1 Compression Test Results for 2 Months of Aging

\begin{tabular}{|c|c|c|c|c|c|c|}
\hline Type & Specimen & $\begin{array}{l}\text { Maximum } \\
\text { Load } \\
\text { lbs. }\end{array}$ & $\begin{array}{l}\text { Maximum } \\
\text { Stress } \\
\text { psi }\end{array}$ & \begin{tabular}{|c} 
Average \\
Max. Stress \\
psi
\end{tabular} & $\begin{array}{l}\text { Reduction in Length } \\
\text { at Max. Stress } \\
\text { in }\end{array}$ & $\begin{array}{l}\text { Stiffness, E } \\
\text { psi } \times 10^{6}\end{array}$ \\
\hline \multirow{10}{*}{ ABS } & CA1-6a & 1120 & 8547 & & 0.058 & - \\
\hline & CA1-6b & 1313 & 9870 & 9208 & 0.080 & - \\
\hline & CA3-4a & 1076 & 8054 & \multirow[b]{2}{*}{8027} & 0.055 & - \\
\hline & CA3-4b & 1069 & 8001 & & 0.046 & - \\
\hline & CA4-4a & 1290 & 9998 & \multirow[b]{2}{*}{10062} & 0.051 & - \\
\hline & CA4-4b & 1360 & 10125 & & 0.045 & - \\
\hline & CA5-6a & 1088 & 8482 & \multirow[b]{2}{*}{8318} & 0.045 & - \\
\hline & CA5-6b & 1048 & 8154 & & 0.050 & - \\
\hline & CA6-5a & 1351 & 10309 & \multirow[b]{2}{*}{10308} & 0.047 & - \\
\hline & CA6-5b & 1356 & 10307 & & 0.047 & - \\
\hline \multirow{12}{*}{$P C$} & CP1-3a & 1294 & 10210 & \multirow[b]{2}{*}{10234} & 0.071 & - \\
\hline & CP1-3b & 1313 & 10259 & & 0.080 & - \\
\hline & CP2-3a & 1587 & 12671 & \multirow[b]{2}{*}{12408} & 0.142 & - \\
\hline & CP2-3b & 1607 & 12146 & & 0.103 & - \\
\hline & CP3-4a & 1257 & 9780 & \multirow[b]{2}{*}{9894} & 0.057 & - \\
\hline & CP3-4b & 1246 & 10008 & & 0.061 & - \\
\hline & CP4-3a & 1617 & 12389 & \multirow[b]{2}{*}{11463} & 0.057 & - \\
\hline & CP4-3b & 1445 & 10538 & & 0.068 & - \\
\hline & CP5-4a & 1285 & 10018 & \multirow[b]{2}{*}{9931} & 0.070 & - \\
\hline & CP5-4b & 1305 & 9844 & & 0.067 & - \\
\hline & CP6-12a & 1544 & 12230 & \multirow[b]{2}{*}{12412} & 0.069 & - \\
\hline & CP6-12b & 1568 & 12595 & & 0.067 & - \\
\hline
\end{tabular}


Table C.2 Compression Test Results for 4 Months of Aging

\begin{tabular}{|c|c|c|c|c|c|c|}
\hline Type & Specimen & $\begin{array}{c}\text { Maximum } \\
\text { Load } \\
\text { lbs. }\end{array}$ & $\begin{array}{c}\text { Maximum } \\
\text { Stress } \\
\text { psi }\end{array}$ & $\begin{array}{c}\text { Average } \\
\text { Max. Stress } \\
\text { psi }\end{array}$ & $\begin{array}{c}\text { Reduction in Length } \\
\text { at Max. Stress } \\
\text { in }\end{array}$ & Stiffness, E \\
psi x 10
\end{tabular}


Table C.3 Compression Test Results for 18 Months of Aging

\begin{tabular}{|c|l|c|c|c|c|c|}
\hline Type & Specimen & $\begin{array}{c}\text { Maximum } \\
\text { Load } \\
\text { Ibs. }\end{array}$ & $\begin{array}{c}\text { Maximum } \\
\text { Stress } \\
\text { psi }\end{array}$ & $\begin{array}{c}\text { Average } \\
\text { Max. Stress } \\
\text { psi }\end{array}$ & $\begin{array}{c}\text { Reduction in Length } \\
\text { at Max. Stress } \\
\text { in }\end{array}$ & Stiffness, E \\
psi x 10
\end{tabular}




\section{APPENDIX D}

IMPACT TEST RESULTS FOR CONDITIONED SPECIMENS

Table D.1 Impact Test Results for 2 Months of Aging

\begin{tabular}{|c|c|c|c|c|c|}
\hline Type & Specimen & $\begin{array}{c}\text { Indicated } \\
\text { Impact Strength }_{\text {ft-lbs }}\end{array}$ & $\begin{array}{c}\text { Corrected } \\
\text { Impact Strength } \\
\text { ft-lbs }\end{array}$ & $\begin{array}{c}\text { Impact Strength } \\
\text { ft-lbs } / \text { in }\end{array}$ & Failure Type \\
\hline \multirow{6}{*}{ ABS } & IA1-7 & 0.51 & 0.46 & 3.67 & $\mathrm{H}$ \\
\hline & IA2-8 & 0.22 & 0.17 & 1.31 & C \\
\hline & IA3-8 & 0.29 & 0.25 & 1.91 & C \\
\hline & IA4-7 & 0.17 & 0.12 & 0.90 & C \\
\hline & IA5-8 & 0.41 & 0.37 & 2.86 & $\mathrm{H}$ \\
\hline & IA6-7 & 0.18 & 0.13 & 1.04 & $C$ \\
\hline
\end{tabular}

Table D.2 Impact Test Results for 4 Months of Aging

\begin{tabular}{|c|c|c|c|c|c|}
\hline Type & Specimen & $\begin{array}{c}\text { Indicated } \\
\text { Impact Strength } \mathrm{ft}^{-\operatorname{lbs}_{\mathrm{f}}}\end{array}$ & $\begin{array}{c}\text { Corrected } \\
\text { Impact Strength } \\
\text { ft-lbs }_{f}\end{array}$ & $\begin{array}{c}\text { Impact Strength } \\
\text { ft-lbs } / \text { in }\end{array}$ & Failure Type \\
\hline \multirow{6}{*}{ ABS } & |A1-8 & 0.52 & 0.47 & 3.76 & $\mathrm{H}$ \\
\hline & IA2-9 & 0.23 & 0.18 & 1.40 & $C$ \\
\hline & IA3-9 & 0.21 & 0.15 & 1.24 & C \\
\hline & IA4-10 & 0.16 & 0.10 & 0.83 & $C$ \\
\hline & IA5-9 & 0.37 & 0.32 & 2.54 & $C$ \\
\hline & IA6-8 & 0.19 & 0.13 & 1.08 & $C$ \\
\hline \multirow{6}{*}{ PC } & IP1-19 & 1.86 & 1.83 & 14.67 & $P$ \\
\hline & IP2-20 & 0.24 & 0.19 & 1.48 & $C$ \\
\hline & IP3-18 & 1.75 & 1.72 & 13.77 & $P$ \\
\hline & IP4-21 & 0.17 & 0.11 & 0.91 & $C$ \\
\hline & IP5-18 & 1.85 & 1.82 & 14.59 & $C$ \\
\hline & IP6-20 & 0.21 & 0.15 & 1.24 & 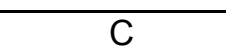 \\
\hline
\end{tabular}


Table D.3 Impact Test Results for 10 Months of Aging

\begin{tabular}{|c|c|c|c|c|c|c|}
\hline Type & Specimen & \begin{tabular}{|c|} 
Indicated \\
Impact Strength \\
ft-lbs
\end{tabular} & $\begin{array}{c}\text { Corrected } \\
\text { Impact Strength }^{\text {ft-lbs }}\end{array}$ & $\begin{array}{c}\text { Impact Strength } \\
\text { ft-lbs } / \text { in }\end{array}$ & $\begin{array}{l}\text { Average Impact } \\
\text { Strength } \\
\text { ft-lbs } f \text { in }\end{array}$ & $\begin{array}{c}\text { Failure } \\
\text { Type }\end{array}$ \\
\hline \multirow{6}{*}{ ABS } & $\begin{array}{l}\text { IA1-7 } \\
\text { IA1-9 } \\
\text { IA } 1-10\end{array}$ & $\begin{array}{l}0.52 \\
0.46 \\
0.46\end{array}$ & $\begin{array}{l}0.47 \\
0.41 \\
0.41\end{array}$ & $\begin{array}{l}3.76 \\
3.27 \\
3.27\end{array}$ & 3.44 & $\begin{array}{l}\mathrm{H} \\
\mathrm{H} \\
\mathrm{H}\end{array}$ \\
\hline & $\begin{array}{c}\text { IA2-4 } \\
\text { IA2-10 }\end{array}$ & $\begin{array}{l}0.22 \\
0.22\end{array}$ & $\begin{array}{l}0.17 \\
0.17\end{array}$ & $\begin{array}{l}1.32 \\
1.32\end{array}$ & 1.32 & $\begin{array}{l}\mathrm{C} \\
\mathrm{C}\end{array}$ \\
\hline & $\begin{array}{c}\text { IA3-4 } \\
\text { IA3-16 }\end{array}$ & $\begin{array}{l}0.16 \\
0.16\end{array}$ & $\begin{array}{l}0.10 \\
0.10\end{array}$ & $\begin{array}{l}0.83 \\
0.83\end{array}$ & 0.83 & $\begin{array}{l}\mathrm{C} \\
\mathrm{C}\end{array}$ \\
\hline & $\begin{array}{l}\text { IA4-7 } \\
\text { IA4-8 }\end{array}$ & $\begin{array}{l}0.15 \\
0.15\end{array}$ & $\begin{array}{l}0.09 \\
0.09\end{array}$ & $\begin{array}{l}0.75 \\
0.75\end{array}$ & 0.75 & $\begin{array}{l}C \\
C\end{array}$ \\
\hline & $\begin{array}{c}\text { IA5-7 } \\
\text { IA5-16 }\end{array}$ & $\begin{array}{l}0.26 \\
0.29\end{array}$ & $\begin{array}{l}0.21 \\
0.24\end{array}$ & $\begin{array}{l}1.65 \\
1.89\end{array}$ & 1.77 & $\begin{array}{l}\mathrm{C} \\
\mathrm{C}\end{array}$ \\
\hline & $\begin{array}{c}\text { IA6-9 } \\
\text { IA6-10 }\end{array}$ & $\begin{array}{l}0.17 \\
0.19\end{array}$ & $\begin{array}{l}0.11 \\
0.13\end{array}$ & $\begin{array}{l}0.91 \\
1.08\end{array}$ & 1.00 & $\begin{array}{l}C \\
C\end{array}$ \\
\hline \multirow{6}{*}{ PC } & IP1-21 & 1.85 & 1.82 & 14.59 & 14.59 & $P$ \\
\hline & IP2-22 & 0.23 & 0.18 & 1.40 & 1.40 & C \\
\hline & IP3-19 & 1.98 & 1.96 & 15.65 & 15.65 & $C$ \\
\hline & IP4-22 & 0.17 & 0.11 & 0.91 & 0.91 & $C$ \\
\hline & $\begin{array}{l}\text { IP5-19 } \\
\end{array}$ & 1.89 & 1.86 & 14.91 & 14.91 & $P$ \\
\hline & IP6-21 & 0.22 & 0.17 & 1.32 & 1.32 & C \\
\hline
\end{tabular}


Table D.4 Impact Test Results for 18 Months of Aging

\begin{tabular}{|c|c|c|c|c|c|c|}
\hline Type & Specimen & \begin{tabular}{|c|} 
Indicated \\
Impact Strength \\
$\mathrm{ft}-\mathrm{Ibs}_{\mathrm{f}}$
\end{tabular} & $\begin{array}{c}\text { Corrected } \\
\text { Impact Strength } \\
\text { ft-lbs }\end{array}$ & $\begin{array}{c}\text { Impact Strength } \\
\text { ft-lbs } / \text { in }\end{array}$ & $\begin{array}{c}\text { Average } \\
\text { Impact Strength } \\
\mathrm{ft}^{-\mathrm{Ibs}_{\mathrm{f}} / \mathrm{in}}\end{array}$ & $\begin{array}{c}\text { Failure } \\
\text { Type }\end{array}$ \\
\hline \multirow{17}{*}{ ABS } & IA1-8 & 0.52 & 0.47 & 3.76 & & $\mathrm{H}$ \\
\hline & $\mid A 1-9$ & 0.45 & 0.40 & 3.19 & & $\mathrm{H}$ \\
\hline & $\mid A 1-10$ & 0.46 & 0.41 & 3.27 & 3.41 & $\mathrm{H}$ \\
\hline & IA2-5 & 0.21 & 0.15 & 1.24 & & $\mathrm{C}$ \\
\hline & IA2-6 & 0.21 & 0.15 & 1.24 & 1.24 & C \\
\hline & IA3-5 & 0.16 & 0.10 & 0.83 & & C \\
\hline & IA3-6 & 0.17 & 0.11 & 0.91 & 0.87 & C \\
\hline & IA4-9 & 0.15 & 0.09 & 0.75 & & C \\
\hline & IA4-10 & 0.15 & 0.09 & 0.75 & 0.75 & $\mathrm{C}$ \\
\hline & IA5-1 & 0.21 & 0.15 & 1.24 & & C \\
\hline & IA5-2 & 0.21 & 0.15 & 1.24 & & C \\
\hline & IA5-3 & 0.22 & 0.17 & 1.32 & & C \\
\hline & IA5-4 & 0.27 & 0.22 & 1.73 & & C \\
\hline & IA5-5 & 0.27 & 0.22 & 1.73 & & C \\
\hline & IA5-6 & 0.24 & 0.19 & 1.48 & 1.46 & C \\
\hline & IA6-6 & 0.16 & 0.10 & 0.83 & & C \\
\hline & IA6-7 & 0.17 & 0.11 & 0.91 & 0.87 & C \\
\hline \multirow{9}{*}{ PC } & $\mathrm{P} 1-21$ & 1.97 & 1.95 & 15.56 & 15.56 & $P$ \\
\hline & P2-18 & 0.23 & 0.18 & 1.40 & & C \\
\hline & $\mathrm{P} 2-22$ & 0.25 & 0.20 & 1.57 & 1.48 & C \\
\hline & P3-17 & 1.90 & 1.87 & 14.99 & 14.99 & $\mathrm{P}$ \\
\hline & P4-20 & 0.16 & 0.10 & 0.83 & & C \\
\hline & P4-22 & 0.15 & 0.09 & 0.75 & 0.79 & C \\
\hline & P5-17 & 1.98 & 1.96 & 15.65 & 15.65 & $\mathrm{P}$ \\
\hline & P6-17 & 0.21 & 0.15 & 1.24 & & C \\
\hline & P6-21 & 0.20 & 0.14 & 1.16 & 1.20 & C \\
\hline
\end{tabular}


APPENDIX E

HARDNESS TEST RESULTS FOR CONDITIONED SPECIMENS

Table E.1 Hardness Test Results for 2 Months of Aging

\begin{tabular}{|c|c|c|}
\hline Type & Specimen & $\begin{array}{c}\text { Hardness } \\
\text { Index }\end{array}$ \\
\hline \multirow{4}{*}{ ABS } & HA1-1 & 12.44 \\
\cline { 2 - 3 } & HA2-1 & 12.80 \\
\cline { 2 - 3 } & HA3-1 & 12.58 \\
\cline { 2 - 3 } & HA4-1 & 11.76 \\
\cline { 2 - 3 } & HA5-1 & 12.44 \\
\cline { 2 - 3 } & HA6-1 & 12.16 \\
\hline \multirow{4}{*}{ PC } & HP1-3 & 12.00 \\
\cline { 2 - 3 } & HP2-3 & 11.10 \\
\cline { 2 - 3 } & HP3-3 & 11.50 \\
\cline { 2 - 3 } & HP4-3 & 11.44 \\
\cline { 2 - 3 } & HP5-4 & 10.74 \\
\cline { 2 - 3 } & HP6-2 & 11.38 \\
\hline
\end{tabular}

Table E.2 Hardness Test Results for 4 Months of Aging

\begin{tabular}{|c|c|c|}
\hline Type & Specimen & $\begin{array}{c}\text { Hardness } \\
\text { Index }\end{array}$ \\
\hline \multirow{4}{*}{ ABS } & HA1-2 & 11.70 \\
\cline { 2 - 3 } & HA2-2 & 11.50 \\
\cline { 2 - 3 } & HA3-2 & 11.40 \\
\cline { 2 - 3 } & HA4-2 & 11.30 \\
\cline { 2 - 3 } & HA5-2 & 10.80 \\
\cline { 2 - 3 } & HA6-2 & 11.00 \\
\hline \multirow{4}{*}{ PC } & HP1-4 & 11.30 \\
\cline { 2 - 3 } & HP2-4 & 11.20 \\
\cline { 2 - 3 } & HP3-4 & 10.80 \\
\cline { 2 - 3 } & HP4-4 & 10.80 \\
\cline { 2 - 3 } & HP5-5 & 11.20 \\
\cline { 2 - 3 } & HP6-3 & 11.40 \\
\hline
\end{tabular}


Table E.3 Hardness Test Results for 10 Months of Aging

\begin{tabular}{|c|c|c|c|}
\hline Type & Specimen & $\begin{array}{l}\text { Hardness } \\
\text { Index }\end{array}$ & $\begin{array}{c}\text { Average } \\
\text { Hardness } \\
\text { Index }\end{array}$ \\
\hline \multirow{12}{*}{$A B S$} & HA1-3 & 12.30 & \\
\hline & HA1-4 & 11.90 & 12.10 \\
\hline & HA2-3 & 10.30 & \\
\hline & HA2-4 & 11.70 & 11.00 \\
\hline & HA3-3 & 11.70 & \\
\hline & HA3-4 & 10.50 & 11.10 \\
\hline & HA4-3 & 10.00 & \\
\hline & HA4-4 & 11.60 & 10.80 \\
\hline & HA5-3 & 11.40 & \\
\hline & HA5-4 & 10.80 & 11.10 \\
\hline & HA6-3 & 10.10 & \\
\hline & HA6-4 & 11.70 & 10.90 \\
\hline \multirow{12}{*}{ PC } & HP1-5 & 10.90 & \\
\hline & HP1-6 & 11.30 & 11.10 \\
\hline & HP2-5 & 10.70 & \\
\hline & HP2-6 & 11.90 & 11.30 \\
\hline & HP3-5 & 10.60 & \\
\hline & HP3-6 & 11.60 & 11.10 \\
\hline & HP4-5 & 11.50 & \\
\hline & HP4-6 & 10.30 & 10.90 \\
\hline & HP5-6 & 11.00 & \\
\hline & HP5-7 & 10.80 & 10.90 \\
\hline & HP6-4 & 10.90 & \\
\hline & HP6-6 & 10.90 & 10.90 \\
\hline
\end{tabular}


Table E.4 Hardness Test Results for 18 Months of Aging

\begin{tabular}{|c|c|c|c|}
\hline Type & Specimen & $\begin{array}{l}\text { Hardness } \\
\text { Index }\end{array}$ & $\begin{array}{c}\text { Average } \\
\text { Hardness } \\
\text { Index }\end{array}$ \\
\hline \multirow{11}{*}{ ABS } & $\begin{array}{l}\text { HA1-3 } \\
\text { HA1-4 }\end{array}$ & $\begin{array}{l}11.80 \\
12.20\end{array}$ & 12.00 \\
\hline & HA2-3 & 10.70 & \\
\hline & HA2-4 & 11.10 & 10.90 \\
\hline & HA3-3 & 11.50 & \\
\hline & HA3-4 & 10.50 & 11.00 \\
\hline & HA4-3 & 11.20 & \\
\hline & HA4-4 & 10.00 & 10.60 \\
\hline & HA5-3 & 10.20 & \\
\hline & HA5-4 & 11.60 & 10.90 \\
\hline & HA6-3 & 11.60 & \\
\hline & HA6-4 & 10.00 & 10.80 \\
\hline \multirow{12}{*}{ PC } & HP1-5 & 10.60 & \\
\hline & HP1-6 & 11.40 & 11.00 \\
\hline & HP2-5 & 11.70 & \\
\hline & HP2-6 & 10.90 & 11.30 \\
\hline & HP3-5 & 11.00 & \\
\hline & HP3-6 & 11.00 & 11.00 \\
\hline & HP4-5 & 10.90 & \\
\hline & HP4-6 & 10.70 & 10.80 \\
\hline & HP5-6 & 10.50 & \\
\hline & HP5-7 & 11.30 & 10.90 \\
\hline & HP6-4 & 10.90 & \\
\hline & HP6-6 & 10.90 & 10.90 \\
\hline
\end{tabular}




\section{APPENDIX F}

\section{COMPARISON CHARTS OF TEST RESULTS ON CONDITIONED RECYCLED COUPONS}

Table F.1 Tensile Strength Variations in Aged ABS Specimens

\begin{tabular}{|c|c|c|c|c|c|c|c|c|}
\hline \multirow{3}{*}{\begin{tabular}{|c|} 
Specimen \\
Type
\end{tabular}} & \multicolumn{5}{|c|}{ Max. Tensile Stress (psi) } & \multicolumn{3}{|c|}{$\%$ Change } \\
\hline & \multicolumn{5}{|c|}{ Months of aging } & \multirow{2}{*}{$\begin{array}{l}\text { End of } \\
\text { period }\end{array}$} & \multirow{2}{*}{$\begin{array}{l}\text { Max gain } \\
\text { in period }\end{array}$} & \multirow{2}{*}{$\begin{array}{l}\text { Max red. } \\
\text { in period }\end{array}$} \\
\hline & $\mathbf{0}$ & 2 & 4 & 10 & 18 & & & \\
\hline A1 & 6174 & 5866 & - & 6118 & 5809 & -5.9 & No gain & -5.9 \\
\hline A2 & 10299 & 9683 & 10510 & 9410 & 10678 & +3.7 & +3.7 & -8.6 \\
\hline A3 & 5578 & 5285 & 5988 & 5549 & 5360 & -3.9 & +7.4 & -5.3 \\
\hline A4 & 8800 & 8802 & 9388 & 7602 & 8637 & -1.9 & +6.7 & -13.6 \\
\hline A5 & 5916 & 5558 & - & 5790 & 5683 & -3.9 & No gain & -6.1 \\
\hline A6 & 8911 & 9607 & 10619 & 9123 & 8916 & +0.1 & +19.2 & No red. \\
\hline \multicolumn{6}{|c|}{ Average reduction (-ve values only) without fibers (A1,A3,A5) } & -4.6 & & \\
\hline \multicolumn{6}{|c|}{ Average change (+ve and -ve values) without fibers $(\mathrm{A} 1, \mathrm{~A} 3, \mathrm{~A} 5)$} & -4.6 & +7.4 & -5.7 \\
\hline \multicolumn{6}{|c|}{ Average reduction (-ve values only) with fibers (A2,A4,A6) } & -1.9 & & \\
\hline \multicolumn{6}{|c|}{ Average change (+ve and -ve values) with fibers $(\mathrm{A} 2, \mathrm{~A} 4, \mathrm{~A} 6)$} & +0.6 & +9.8 & -11.1 \\
\hline
\end{tabular}

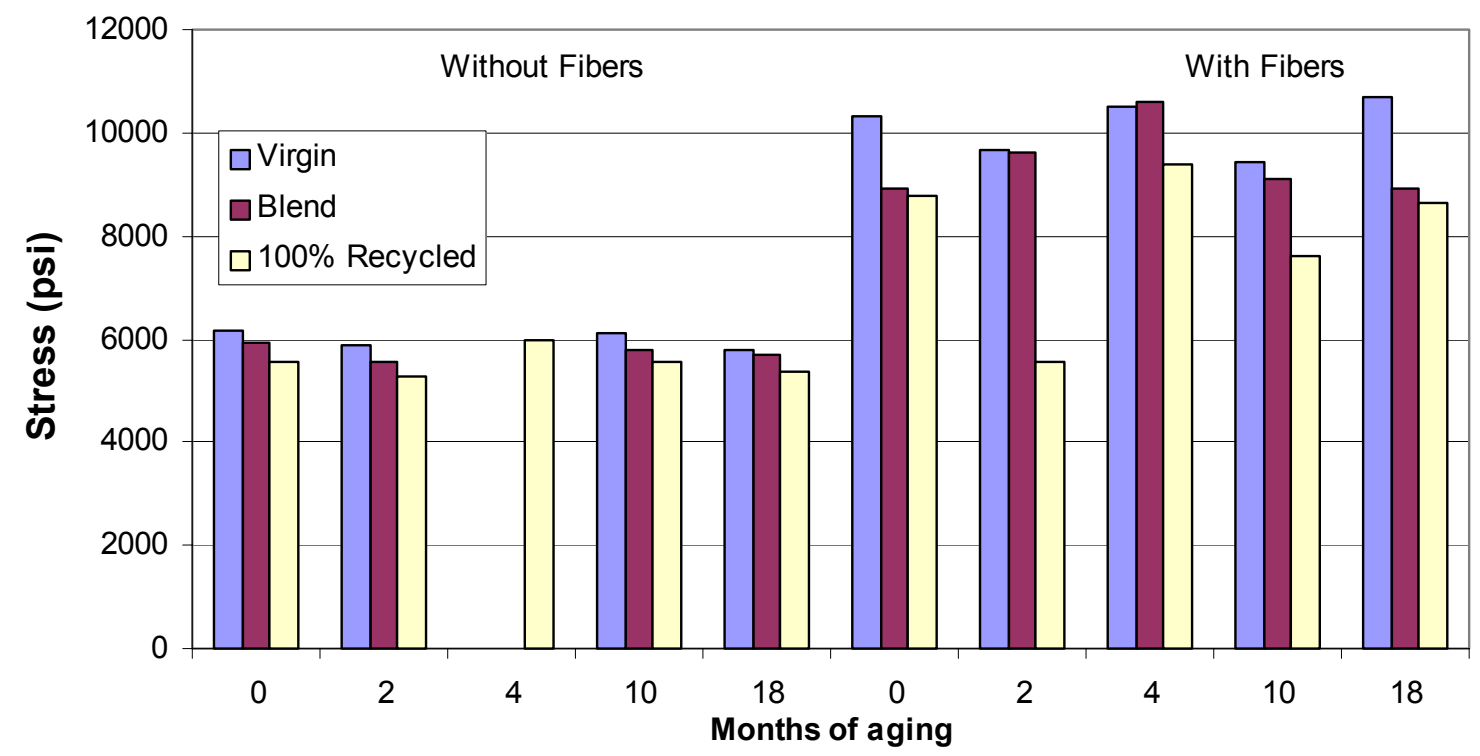

Note: Some intermittent results betw een 0 and 18 months may not be present due to no testing during that period

Figure F.1 Tensile Strength in Aged ABS Specimens 
Table F.2 Tensile Stiffness Variations in Aged ABS Specimens

\begin{tabular}{|c|c|c|c|c|c|c|c|c|}
\hline \multirow{3}{*}{$\begin{array}{c}\text { Specimen } \\
\text { Type }\end{array}$} & \multicolumn{5}{|c|}{ Tensile Stiffness (psi x10 } & \multicolumn{3}{|c|}{ \% Change } \\
\hline & \multicolumn{5}{|c|}{ Months of aging } & \multirow{2}{*}{$\begin{array}{l}\text { End of } \\
\text { period }\end{array}$} & \multirow{2}{*}{\begin{tabular}{|l|} 
Max gain \\
in period
\end{tabular}} & \multirow{2}{*}{$\begin{array}{l}\text { Max red. } \\
\text { in period }\end{array}$} \\
\hline & $\mathbf{0}$ & 2 & 4 & $\mathbf{1 0}$ & $\mathbf{1 8}$ & & & \\
\hline $\mathrm{A} 1$ & 0.321 & - & 0.510 & 0.360 & 0.362 & +12.8 & +58.9 & No red. \\
\hline A2 & 0.976 & - & 1.019 & 1.047 & 1.027 & +5.2 & +7.3 & No red. \\
\hline A3 & 0.333 & - & - & 0.337 & 0.331 & -0.6 & +1.2 & -0.6 \\
\hline A4 & 0.964 & - & - & 0.909 & 1.012 & +5.0 & +5.0 & -5.7 \\
\hline A5 & 0.359 & - & 0.360 & 0.347 & 0.351 & -2.2 & +0.3 & -3.3 \\
\hline A6 & 0.941 & - & 1.050 & 0.993 & 1.041 & +10.6 & +11.6 & No red. \\
\hline \multicolumn{6}{|c|}{ Average reduction (-ve values only) without fibers $(\mathrm{A} 1, \mathrm{~A} 3, \mathrm{~A} 5)$} & -1.4 & & \\
\hline \multicolumn{6}{|c|}{ Average change (+ve and -ve values) without fibers $(\mathrm{A} 1, \mathrm{~A} 3, \mathrm{~A} 5)$} & +3.3 & +20.1 & -2.0 \\
\hline \multicolumn{6}{|c|}{ Average reduction (-ve values only) with fibers (A2,A4,A6) } & 0 & & \\
\hline \multicolumn{6}{|c|}{ Average change (+ve and $-\mathrm{ve}$ values) with fibers $(\mathrm{A} 2, \mathrm{~A} 4, \mathrm{~A} 6)$} & +6.9 & +7.9 & -5.7 \\
\hline
\end{tabular}

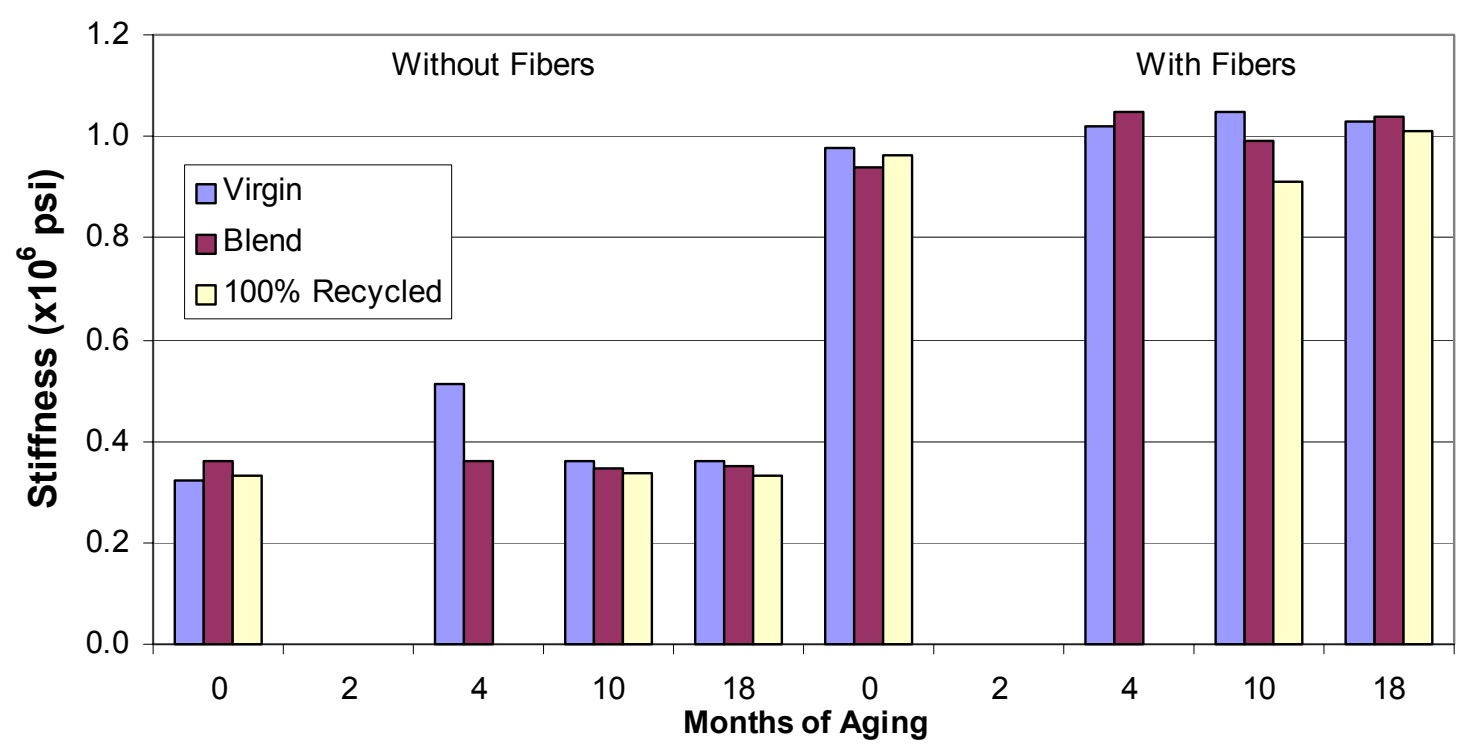

Note: Some intermittent results betw een 0 and 18 months may not be present due to no testing during that period

Figure F.2 Tensile Stiffness in Aged ABS Specimens 
Table F.3 Tensile Strength Variations in Aged PC Specimens

\begin{tabular}{|c|c|c|c|c|c|c|c|c|}
\hline \multirow{3}{*}{$\begin{array}{c}\text { Specimen } \\
\text { Type }\end{array}$} & \multicolumn{5}{|c|}{ Max. Tensile Stress (psi) } & \multicolumn{3}{|c|}{ \% Change } \\
\hline & \multicolumn{5}{|c|}{ Months of aging } & \multirow{2}{*}{$\begin{array}{l}\text { End of } \\
\text { period }\end{array}$} & \multirow{2}{*}{$\begin{array}{l}\text { Max gain } \\
\text { in period }\end{array}$} & \multirow{2}{*}{$\begin{array}{l}\text { Max red } \\
\text { in period }\end{array}$} \\
\hline & $\mathbf{0}$ & 2 & 4 & 10 & 18 & & & \\
\hline P1 & 8664 & 8210 & 9261 & 8817 & 8352 & -3.6 & +6.9 & -5.2 \\
\hline $\mathrm{P} 2$ & 16479 & 15101 & 15721 & 12867 & 11892 & -27.8 & No gain & -27.8 \\
\hline P3 & 8566 & 7813 & 9233 & 8983 & 8329 & -2.8 & +7.8 & -8.8 \\
\hline P4 & 15213 & 14203 & 14205 & 12525 & 10920 & -28.2 & No gain & -28.2 \\
\hline P5 & 8926 & 8072 & 9163 & 8589 & 8185 & -8.3 & +2.7 & -9.6 \\
\hline P6 & 16555 & 14831 & - & 12877 & 11848 & -28.4 & No gain & -28.4 \\
\hline \multicolumn{6}{|c|}{ Average reduction (-ve values only) without fibers $(\mathrm{A} 1, \mathrm{~A} 3, \mathrm{~A} 5)$} & -4.9 & & \\
\hline \multicolumn{6}{|c|}{ Average change (+ve and -ve values) without fibers $(\mathrm{A} 1, \mathrm{~A} 3, \mathrm{~A} 5)$} & -4.9 & +5.8 & -7.9 \\
\hline \multicolumn{6}{|c|}{ Average reduction (-ve values only) with fibers (A2,A4,A6) } & -28.2 & & \\
\hline \multicolumn{6}{|c|}{ Average change (+ve and $-\mathrm{ve}$ values) with fibers $(\mathrm{A} 2, \mathrm{~A} 4, \mathrm{~A} 6)$} & -28.2 & No gain & -28.2 \\
\hline
\end{tabular}

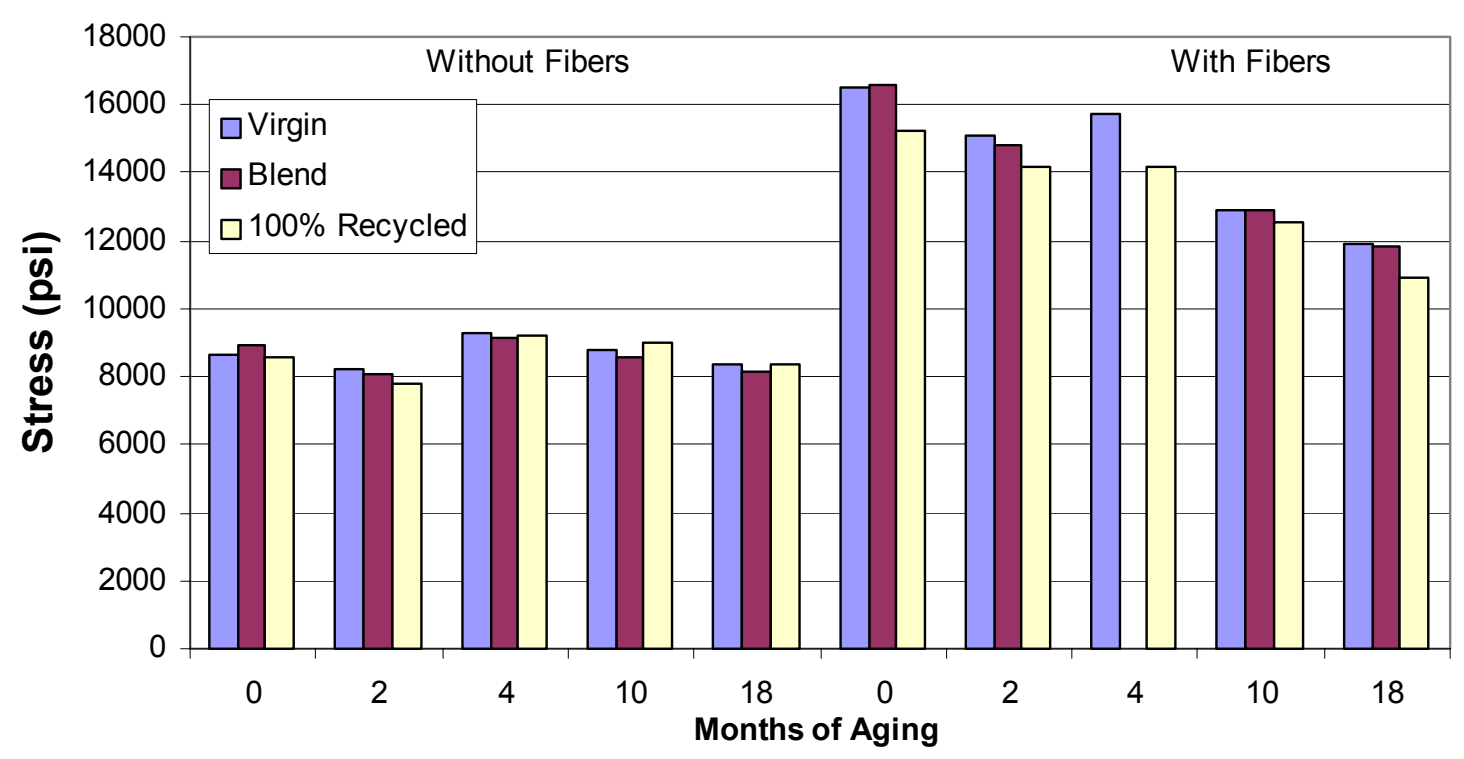

Note: Some intermittent results betw een 0 and 18 months may not be present due to no testing during that period

Figure F.3 Tensile Strength in Aged PC Specimens 
Table F.4 Tensile Stiffness Variations in Aged PC Specimens

\begin{tabular}{|c|c|c|c|c|c|c|c|c|}
\hline \multirow{3}{*}{\begin{tabular}{|c} 
Specimen \\
Type
\end{tabular}} & \multicolumn{5}{|c|}{ Tension Stiffness (psi x10 } & \multicolumn{3}{|c|}{ \% Change } \\
\hline & \multicolumn{5}{|c|}{ Months of aging } & \multirow{2}{*}{$\begin{array}{l}\text { End of } \\
\text { period }\end{array}$} & \multirow{2}{*}{$\begin{array}{l}\text { Max gain } \\
\text { in period }\end{array}$} & \multirow{2}{*}{$\begin{array}{l}\text { Max red } \\
\text { in period }\end{array}$} \\
\hline & $\mathbf{0}$ & 2 & 4 & 10 & 18 & & & \\
\hline P1 & 0.300 & - & 0.309 & 0.364 & 0.35 & +16.7 & +21.3 & No red. \\
\hline $\mathrm{P} 2$ & 1.029 & - & 1.008 & 1.076 & 1.068 & +3.8 & +4.6 & -2.0 \\
\hline P3 & 0.293 & - & 0.315 & 0.364 & 0.343 & +17.1 & +24.2 & No red. \\
\hline P4 & 0.993 & - & 0.964 & 1.061 & 1.073 & +8.1 & +8.1 & -2.9 \\
\hline P5 & 0.329 & - & 0.337 & 0.347 & 0.349 & +6.1 & +6.1 & No red. \\
\hline P6 & 1.046 & - & - & 1.099 & 1.091 & +4.3 & +5.1 & No red. \\
\hline \multicolumn{6}{|c|}{ Average reduction (-ve values only) without fibers $(\mathrm{A} 1, \mathrm{~A} 3, \mathrm{~A} 5)$} & 0 & & \\
\hline \multicolumn{6}{|c|}{ Average change (+ve and -ve values) without fibers $(\mathrm{A} 1, \mathrm{~A} 3, \mathrm{~A} 5)$} & +13.3 & +17.2 & No red. \\
\hline \multicolumn{6}{|c|}{ Average reduction (-ve values only) with fibers (A2,A4,A6) } & 0 & & \\
\hline \multicolumn{6}{|c|}{ Average change (+ve and -ve values) with fibers $(\mathrm{A} 2, \mathrm{~A} 4, \mathrm{~A} 6)$} & +5.4 & +5.9 & -2.5 \\
\hline
\end{tabular}

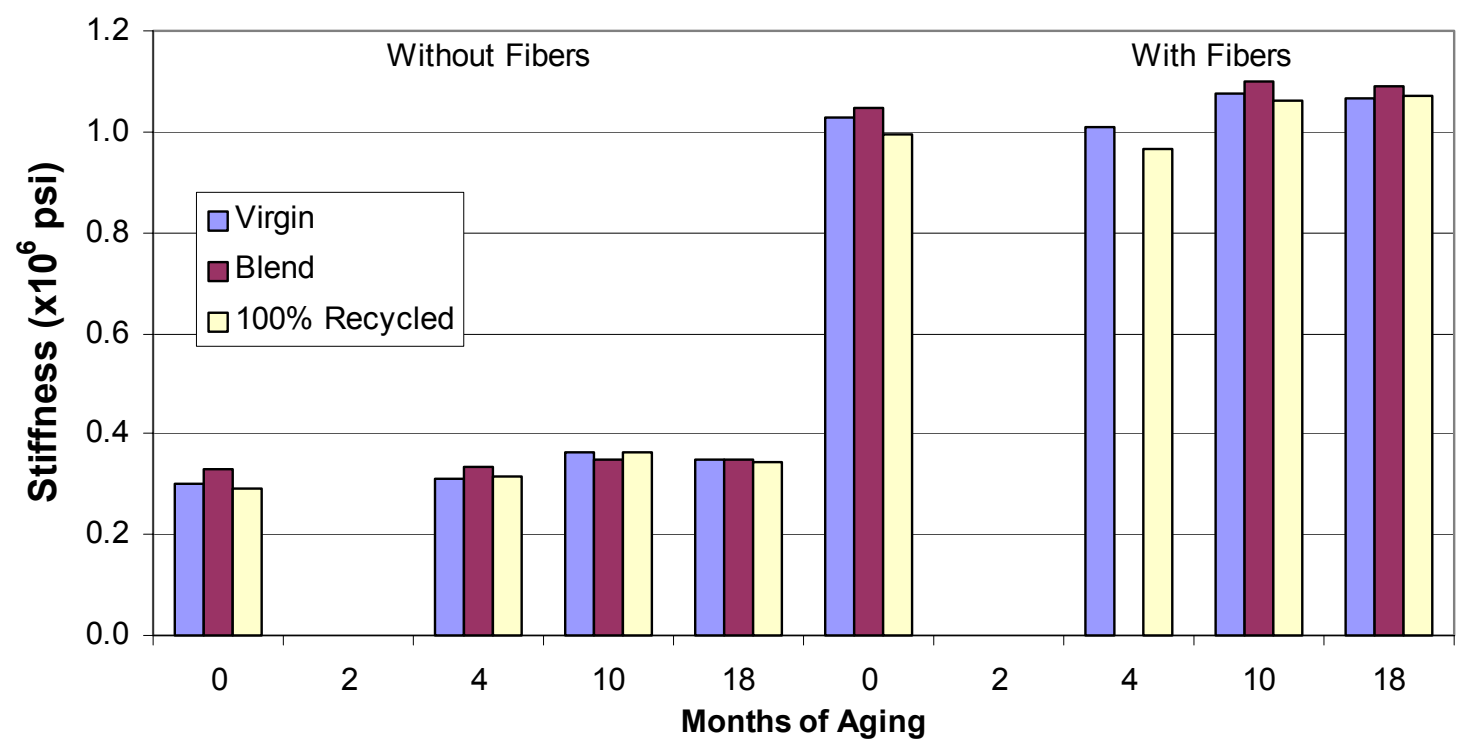

Note: Some intermittent results betw een 0 and 18 months may not be present due to no testing during that period

Figure F.4 Tensile Stiffness in Aged PC Specimens 
Table F.5 Bending Strength Variations in Aged ABS Specimens

\begin{tabular}{|c|c|c|c|c|c|c|c|c|}
\hline \multirow{3}{*}{\begin{tabular}{|c|} 
Specimen \\
Type
\end{tabular}} & \multicolumn{5}{|c|}{ Max. Bending Stress (psi) } & \multicolumn{3}{|c|}{$\%$ Change } \\
\hline & \multicolumn{5}{|c|}{ Months of aging } & \multirow{2}{*}{$\begin{array}{l}\text { End of } \\
\text { period }\end{array}$} & \multirow{2}{*}{$\begin{array}{l}\text { Max gain } \\
\text { in period }\end{array}$} & \multirow{2}{*}{$\begin{array}{l}\text { Max red. } \\
\text { in period }\end{array}$} \\
\hline & $\mathbf{0}$ & 2 & 4 & 10 & 18 & & & \\
\hline A1 & 9080 & - & 6728 & 10747 & 8971 & -3.2 & +18.4 & -25.9 \\
\hline A2 & 17771 & 16033 & 15211 & 14631 & 14080 & -20.8 & No gain & -20.8 \\
\hline A3 & 9187 & 8931 & 7862 & 8923 & 8511 & -7.4 & No gain & -14.4 \\
\hline A4 & 16740 & 15522 & 13886 & 12162 & 12605 & -24.7 & No gain & -27.3 \\
\hline A5 & 9558 & 9295 & 8412 & 10446 & 9419 & -1.5 & +9.3 & -12.0 \\
\hline A6 & 16509 & 17793 & 14043 & 11400 & 13034 & -21.0 & No gain & -30.9 \\
\hline \multicolumn{6}{|c|}{ Average reduction (-ve values only) without fibers (A1,A3,A5) } & -4.0 & & \\
\hline \multicolumn{6}{|c|}{ Average change (+ve and -ve values) without fibers (A1,A3,A5) } & -4.0 & +13.8 & -17.4 \\
\hline \multicolumn{6}{|c|}{ Average reduction (-ve values only) with fibers (A2,A4,A6) } & -22.2 & & \\
\hline \multicolumn{6}{|c|}{ Average change (+ve and -ve values) with fibers $(\mathrm{A} 2, \mathrm{~A} 4, \mathrm{~A} 6)$} & -22.2 & No gain & -26.4 \\
\hline
\end{tabular}

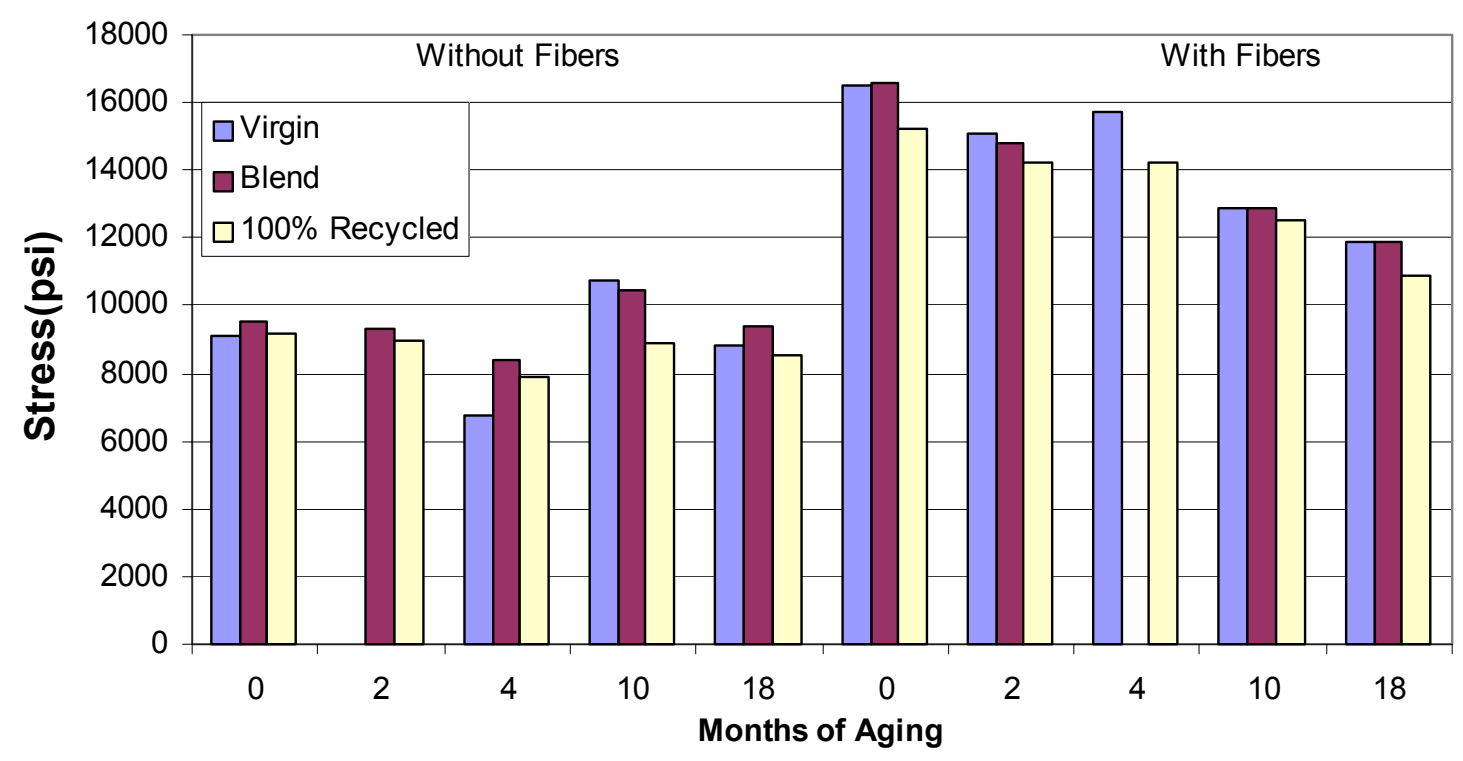

Note: Some intermittent results betw een 0 and 18 months may not be present due to no testing during that period

Figure F.5 Bending Strength in Aged ABS Specimens 
Table F.6 Bending Stiffness Variations in Aged ABS Specimens

\begin{tabular}{|c|c|c|c|c|c|c|c|c|}
\hline \multirow{2}{*}{$\begin{array}{c}\text { Specimen } \\
\text { Type }\end{array}$} & \multicolumn{4}{|c|}{ Bending Stiffness (psi x10 } & \multicolumn{3}{c|}{ \% Change } \\
\cline { 2 - 9 } & \multicolumn{4}{|c|}{ Months of aging } & $\begin{array}{c}\text { End of } \\
\text { period }\end{array}$ & $\begin{array}{c}\text { Max gain } \\
\text { in period }\end{array}$ & $\begin{array}{c}\text { Max red. } \\
\text { in period }\end{array}$ \\
\cline { 2 - 9 } & $\mathbf{0}$ & $\mathbf{2}$ & $\mathbf{4}$ & $\mathbf{1 0}$ & $\mathbf{1 8}$ & -4.8 & +74.8 & -16.5 \\
\hline A1 & 0.40 & - & 0.334 & 0.699 & 0.381 & -5.9 & +19.1 & -8.6 \\
\hline A2 & 1.02 & - & 0.932 & 1.215 & 0.960 & -5.9 \\
\hline A3 & 0.40 & - & 0.364 & 0.408 & 0.391 & -2.3 & +2.0 & -9.0 \\
\hline A4 & 1.08 & - & 0.930 & 0.998 & 0.996 & -7.8 & No gain & -13.9 \\
\hline A5 & 0.40 & - & 0.346 & 0.385 & 0.395 & -1.3 & No gain & -13.5 \\
\hline A6 & 1.11 & - & 0.917 & 0.925 & 0.962 & -13.3 & No gain & -17.4 \\
\hline Average reduction (-ve values only) without fibers (A1,A3,A5) & -2.8 & & \\
\hline Average change (+ve and -ve values) without fibers (A1,A3,A5) & -2.8 & +38.4 & -13.0 \\
\hline Average reduction (-ve values only) with fibers (A2,A4,A6) & -9.0 & & \\
\hline
\end{tabular}

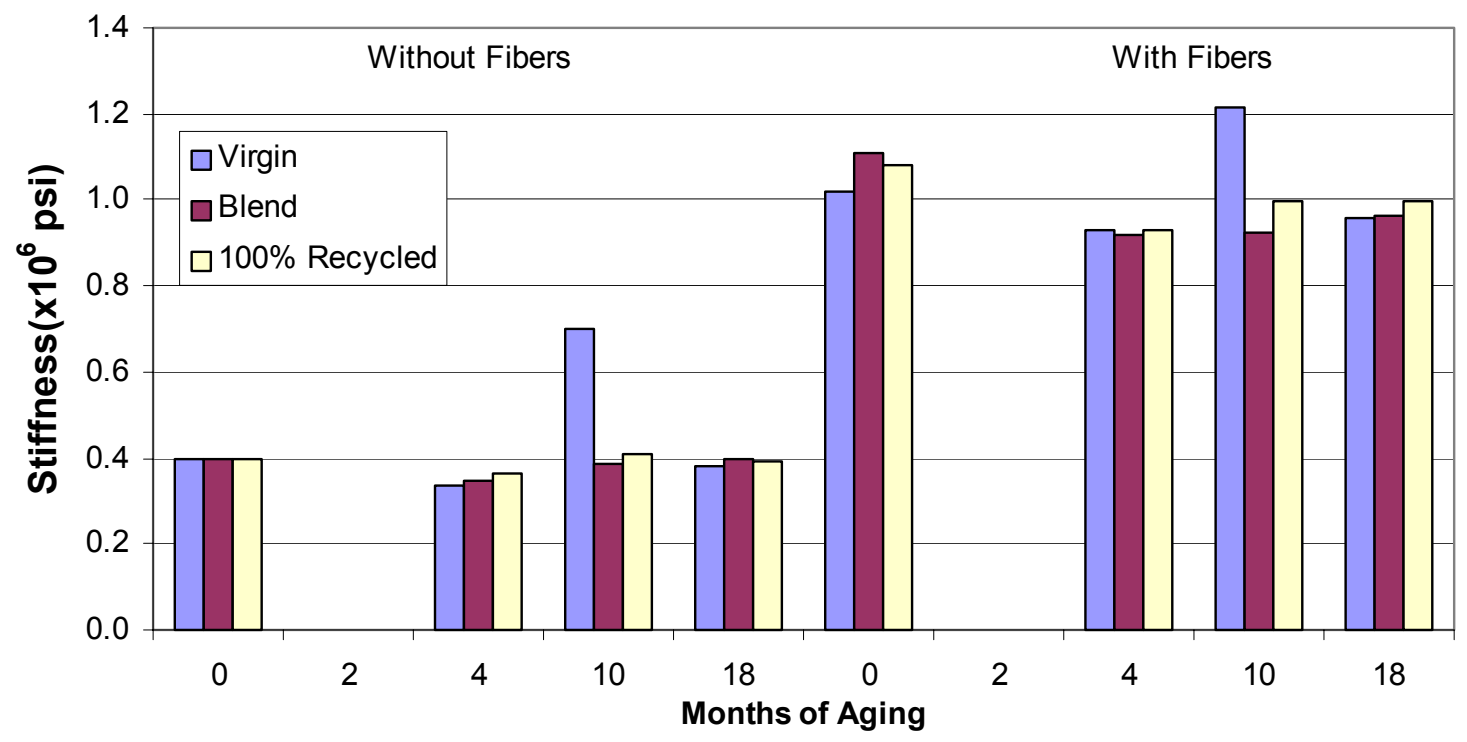

Note: Some intermittent results betw een 0 and 18 months may not be present due to no testing during that period

Figure F.6 Bending Stiffness in Aged ABS Specimens 
Table F.7 Bending Strength Variations in Aged PC Specimens

\begin{tabular}{|c|c|c|c|c|c|c|c|c|}
\hline \multirow{3}{*}{$\begin{array}{c}\text { Specimen } \\
\text { Type }\end{array}$} & \multicolumn{5}{|c|}{ Max. Bending Stress (psi) } & \multicolumn{3}{|c|}{ \% Change } \\
\hline & \multicolumn{5}{|c|}{ Months of aging } & \multirow{2}{*}{$\begin{array}{l}\text { End of } \\
\text { period }\end{array}$} & \multirow{2}{*}{$\begin{array}{l}\text { Max gain } \\
\text { in period }\end{array}$} & \multirow{2}{*}{$\begin{array}{l}\text { Max red } \\
\text { in period }\end{array}$} \\
\hline & $\mathbf{0}$ & 2 & 4 & 10 & 18 & & & \\
\hline P1 & 10242 & 7494 & 9283 & 14945 & 12506 & +22.1 & +45.9 & -26.8 \\
\hline P2 & 24566 & 20228 & 21336 & 25370 & 19908 & -19.0 & +3.3 & -19.0 \\
\hline P3 & 10363 & 8235 & 8398 & 17327 & 12117 & +16.9 & +67.2 & -20.5 \\
\hline $\mathrm{P} 4$ & 23198 & 18830 & 17117 & 28586 & 19798 & -14.7 & +23.2 & -26.2 \\
\hline P5 & 11168 & 10136 & 10602 & 15023 & 12366 & +10.7 & +34.5 & -9.2 \\
\hline P6 & 24886 & 19606 & 21159 & 23909 & 20441 & -17.9 & No gain & -21.2 \\
\hline \multicolumn{6}{|c|}{ Average reduction (-ve values only) without fibers $(\mathrm{A} 1, \mathrm{~A} 3, \mathrm{~A} 5)$} & 0 & & \\
\hline \multicolumn{6}{|c|}{ Average change (+ve and -ve values) without fibers $(\mathrm{A} 1, \mathrm{~A} 3, \mathrm{~A} 5)$} & +16.6 & +49.2 & -18.9 \\
\hline \multicolumn{6}{|c|}{ Average reduction (-ve values only) with fibers (A2,A4,A6) } & -17.2 & & \\
\hline \multicolumn{6}{|c|}{ Average change (+ve and -ve values) with fibers $(\mathrm{A} 2, \mathrm{~A} 4, \mathrm{~A} 6)$} & -17.2 & +13.2 & -22.1 \\
\hline
\end{tabular}

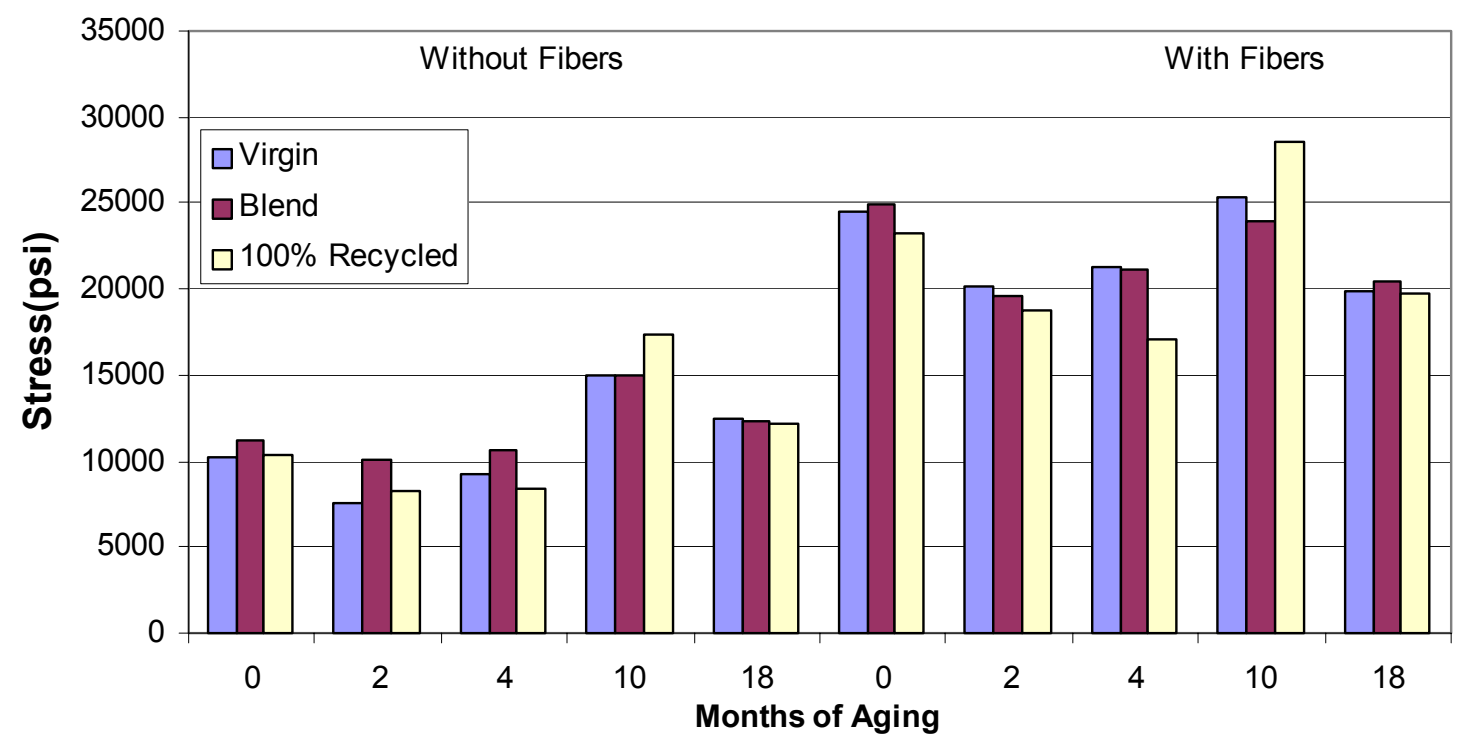

Figure F.7 Bending Strength in Aged PC Specimens 
Table F.8 Bending Stiffness Variations in Aged PC Specimens

\begin{tabular}{|c|c|c|c|c|c|c|c|c|}
\hline \multirow{3}{*}{$\begin{array}{c}\text { Specimen } \\
\text { Type }\end{array}$} & \multicolumn{5}{|c|}{ Bending Stiffness (psi x10 ${ }^{6}$ ) } & \multicolumn{3}{|c|}{ \% Change } \\
\hline & \multicolumn{5}{|c|}{ Months of aging } & \multirow{2}{*}{$\begin{array}{l}\text { End of } \\
\text { period }\end{array}$} & \multirow{2}{*}{$\begin{array}{l}\text { Max gain } \\
\text { in period }\end{array}$} & \multirow{2}{*}{$\begin{array}{l}\text { Max red } \\
\text { in period }\end{array}$} \\
\hline & $\mathbf{0}$ & 2 & 4 & 10 & 18 & & & \\
\hline P1 & 0.332 & - & 0.341 & 0.416 & 0.408 & +22.9 & +25.3 & No red. \\
\hline $\mathrm{P} 2$ & 0.967 & - & 1.001 & 1.089 & 1.031 & +6.6 & +12.6 & No red. \\
\hline P3 & 0.291 & - & 0.354 & 0.445 & 0.450 & +54.6 & +54.6 & No red. \\
\hline $\mathrm{P} 4$ & 0.916 & - & 0.994 & 1.001 & 1.037 & +13.2 & +13.2 & No red. \\
\hline P5 & 0.327 & - & 0.406 & 0.452 & 0.430 & +31.5 & +38.2 & No red. \\
\hline P6 & 0.993 & - & 1.062 & 1.168 & 1.027 & +3.4 & +17.6 & No red. \\
\hline \multicolumn{6}{|c|}{ Average reduction (-ve values only) without fibers (A1,A3,A5) } & 0 & & \\
\hline \multicolumn{6}{|c|}{ Average change (+ve and -ve values) without fibers $(\mathrm{A} 1, \mathrm{~A} 3, \mathrm{~A} 5)$} & +36.3 & +39.4 & No red. \\
\hline \multicolumn{6}{|c|}{ Average reduction (-ve values only) with fibers (A2,A4,A6) } & 0 & & \\
\hline \multicolumn{6}{|c|}{ Average change (+ve and -ve values) with fibers $(\mathrm{A} 2, \mathrm{~A} 4, \mathrm{~A} 6)$} & +7.8 & +14.5 & No red. \\
\hline
\end{tabular}

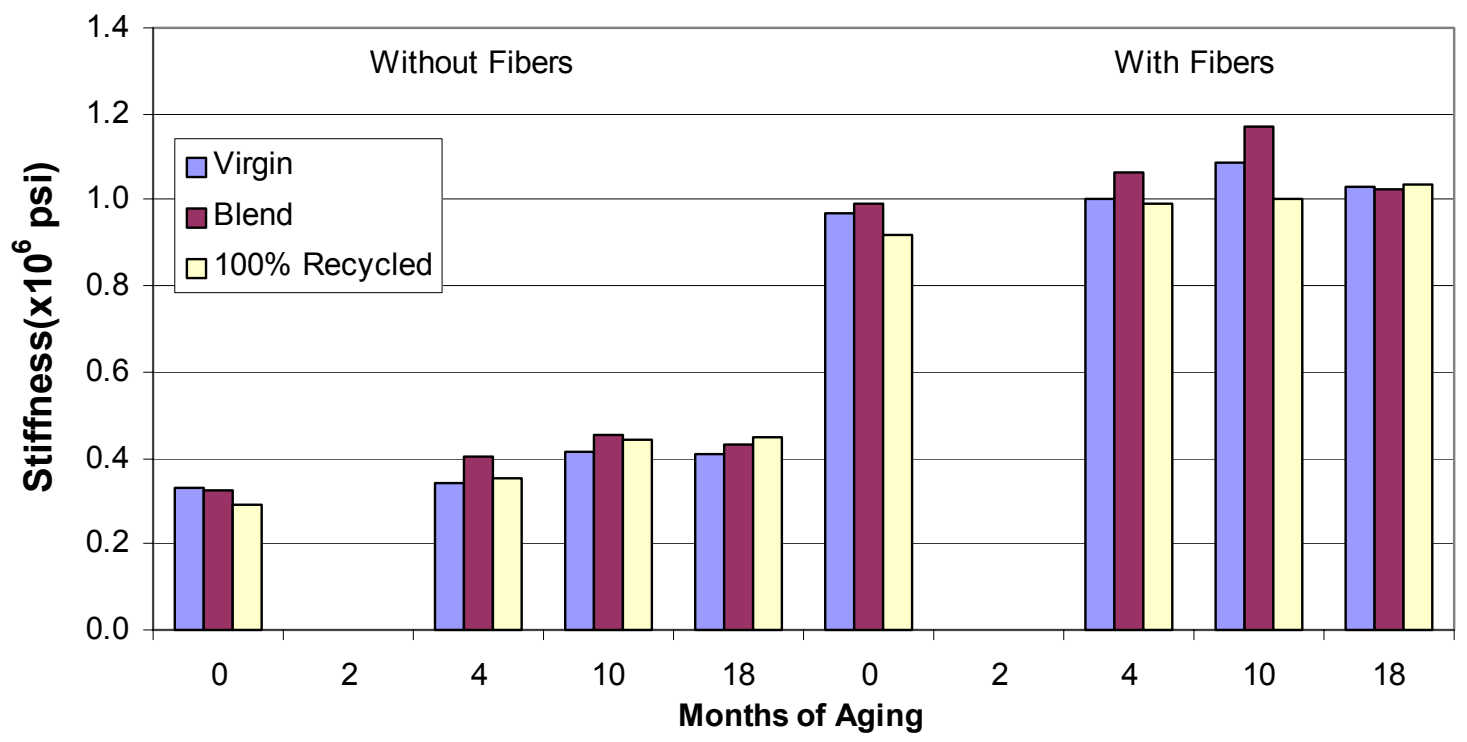

Note: Some intermittent results betw een 0 and 18 months may not be present due to no testing during that period

Figure F.8 Bending Stiffness in Aged PC Specimens 
Table F.9 Compressive Strength Variations in Aged ABS Specimens

\begin{tabular}{|c|c|c|c|c|c|c|c|c|}
\hline \multirow{3}{*}{$\begin{array}{c}\text { Specimen } \\
\text { Type }\end{array}$} & \multicolumn{5}{|c|}{ Max. Compressive Stress (psi) } & \multicolumn{3}{|c|}{ \% Change } \\
\hline & \multicolumn{5}{|c|}{ Months of aging } & \multirow{2}{*}{$\begin{array}{l}\text { End of } \\
\text { period }\end{array}$} & \multirow{2}{*}{$\begin{array}{l}\text { Max gain } \\
\text { in period }\end{array}$} & \multirow{2}{*}{$\begin{array}{l}\text { Max red. } \\
\text { in period }\end{array}$} \\
\hline & $\mathbf{0}$ & 2 & 4 & 10 & 18 & & & \\
\hline $\mathrm{A} 1$ & 8966 & 9208 & 9886 & - & 8244 & -8.1 & +10.3 & -8.1 \\
\hline $\mathrm{A} 2$ & 13135 & - & 11698 & - & 10376 & -21.0 & No gain & -21.0 \\
\hline $\mathrm{A} 3$ & 8792 & 8027 & 8670 & - & 8381 & -4.7 & No gain & -8.7 \\
\hline A4 & 14891 & 10061 & 12440 & - & 10120 & -32.0 & No gain & -32.4 \\
\hline A5 & 8972 & 8318 & 9287 & - & 9011 & +0.4 & +3.5 & -7.3 \\
\hline A6 & 13920 & 10308 & 11151 & - & 11265 & -19.1 & No gain & -25.9 \\
\hline \multicolumn{6}{|c|}{ Average reduction (-ve values only) without fibers (A1,A3,A5) } & -6.4 & & \\
\hline \multicolumn{6}{|c|}{ Average change (+ve and -ve values) without fibers $(\mathrm{A} 1, \mathrm{~A} 3, \mathrm{~A} 5)$} & -4.1 & +6.9 & -8.0 \\
\hline \multicolumn{6}{|c|}{ Average reduction (-ve values only) with fibers (A2,A4,A6) } & -24.0 & & \\
\hline \multicolumn{6}{|c|}{ Average change (+ve and -ve values) with fibers $(\mathrm{A} 2, \mathrm{~A} 4, \mathrm{~A} 6)$} & -24.0 & No gain & -26.5 \\
\hline
\end{tabular}

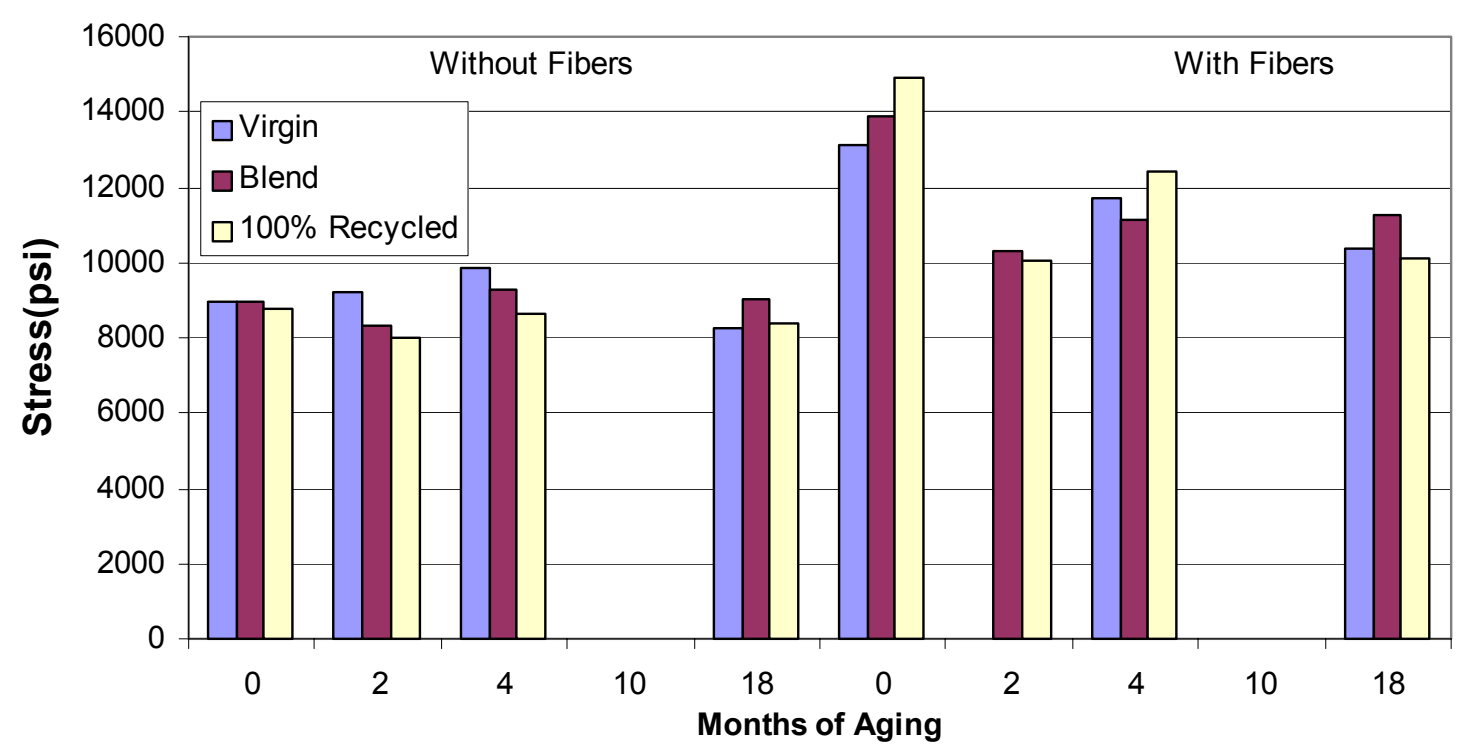

Note: Some intermittent results betw een 0 and 18 months may not be present due to no testing during that period

Figure F.9 Compressive Strength in Aged ABS Specimens 
Table F.10 Compressive Stiffness Variations in Aged ABS Specimens

\begin{tabular}{|c|c|c|c|c|c|c|c|c|}
\hline \multirow{3}{*}{\begin{tabular}{|c|} 
Specimen \\
Type
\end{tabular}} & \multicolumn{5}{|c|}{ Compressive Stiffness (psi) } & \multicolumn{3}{|c|}{$\%$ Change } \\
\hline & \multicolumn{5}{|c|}{ Months of aging } & \multirow{2}{*}{$\begin{array}{l}\text { End of } \\
\text { period }\end{array}$} & \multirow{2}{*}{\begin{tabular}{|l|} 
Max gain \\
in period
\end{tabular}} & \multirow{2}{*}{$\begin{array}{l}\text { Max red. } \\
\text { in period }\end{array}$} \\
\hline & $\mathbf{0}$ & 2 & 4 & 10 & 18 & & & \\
\hline A1 & 0.34 & - & - & - & 0.397 & +16.8 & +16.8 & No red. \\
\hline $\mathrm{A} 2$ & 0.91 & - & - & - & 0.943 & +3.6 & +3.6 & No red. \\
\hline A3 & 0.42 & - & - & - & 0.583 & +38.8 & +38.8 & No red. \\
\hline$\overline{\mathrm{A} 4}$ & 0.92 & - & - & - & 0.600 & -34.8 & No gain & -34.8 \\
\hline A5 & 0.41 & - & - & - & 0.330 & -19.5 & No gain & -19.5 \\
\hline A6 & 1.02 & - & - & - & 0.709 & -30.5 & No gain & -30.5 \\
\hline \multicolumn{6}{|c|}{ Average reduction (-ve values only) without fibers (A1,A3,A5) } & -19.5 & & \\
\hline \multicolumn{6}{|c|}{ Average change (+ve and -ve values) without fibers $(\mathrm{A} 1, \mathrm{~A} 3, \mathrm{~A} 5)$} & +12.0 & +27.8 & -19.5 \\
\hline \multicolumn{6}{|c|}{ Average reduction (-ve values only) with fibers $(\mathrm{A} 2, \mathrm{~A} 4, \mathrm{~A} 6)$} & -32.6 & & \\
\hline \multicolumn{6}{|c|}{ Average change (+ve and -ve values) with fibers $(\mathrm{A} 2, \mathrm{~A} 4, \mathrm{~A} 6)$} & -20.5 & +3.6 & -32.6 \\
\hline
\end{tabular}

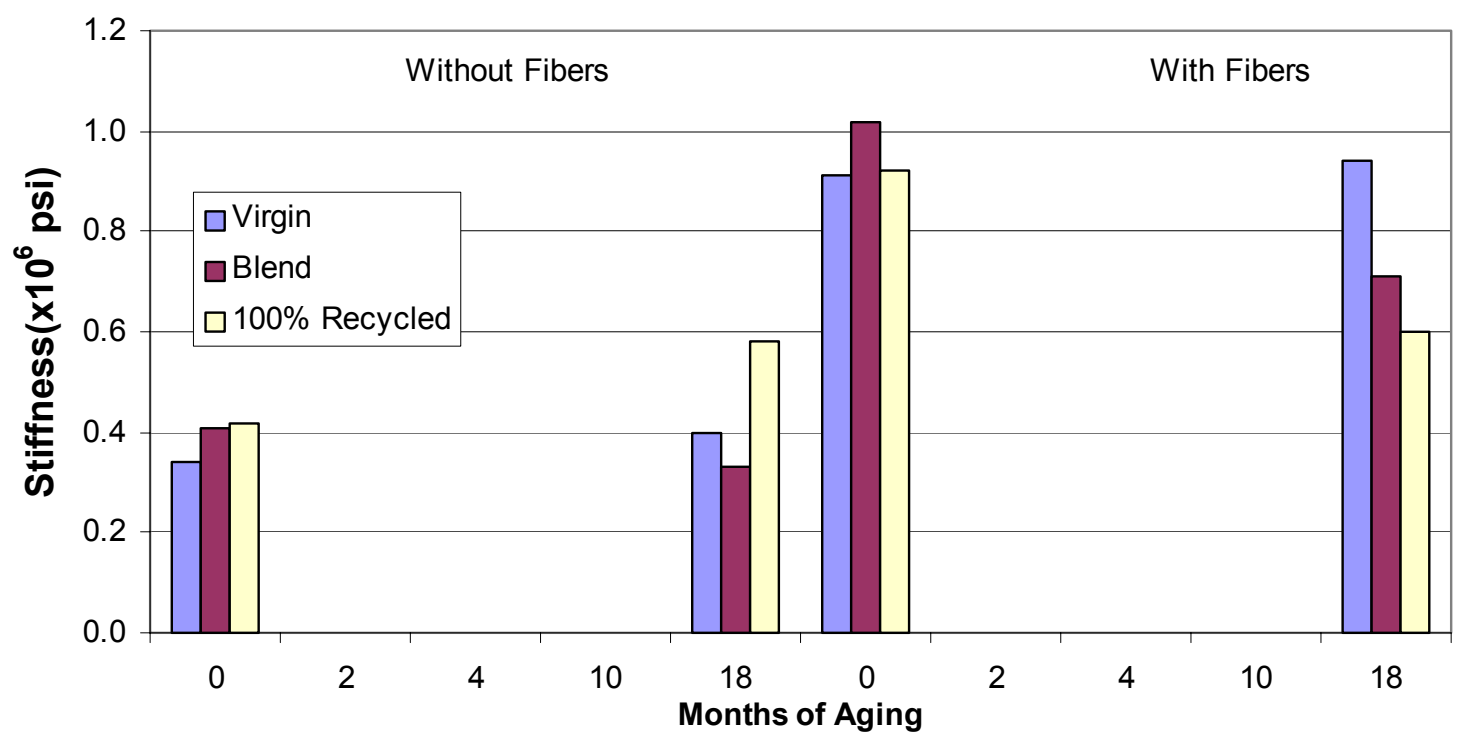

Note: Some intermittent results betw een 0 and 18 months may not be present due to no testing during that period

Figure F.10 Compressive Stiffness in Aged ABS Specimens 
Table F.11 Compressive Strength Variations in Aged PC Specimens

\begin{tabular}{|c|c|c|c|c|c|c|c|c|}
\hline \multirow{3}{*}{\begin{tabular}{|c} 
Specimen \\
Type
\end{tabular}} & \multicolumn{5}{|c|}{ Max. Compressive Stress (psi) } & \multicolumn{3}{|c|}{ \% Change } \\
\hline & \multicolumn{5}{|c|}{ Months of aging } & \multirow{2}{*}{$\begin{array}{l}\text { End of } \\
\text { period }\end{array}$} & \multirow{2}{*}{$\begin{array}{l}\text { Max gain } \\
\text { in period }\end{array}$} & \multirow{2}{*}{$\begin{array}{l}\text { Max red. } \\
\text { in period }\end{array}$} \\
\hline & $\mathbf{0}$ & 2 & 4 & 10 & 18 & & & \\
\hline P1 & 10423 & 10234 & 10259 & - & 10344 & -0.8 & No gain & -1.8 \\
\hline $\mathrm{P} 2$ & 20332 & 12408 & 13539 & - & 12587 & -38.1 & No gain & -39.0 \\
\hline P3 & 10311 & 9894 & 11348 & - & 10296 & -0.1 & +10.1 & -4.0 \\
\hline P4 & 20747 & 11463 & 13509 & - & 13749 & -33.7 & No gain & -44.7 \\
\hline P5 & 10523 & 9931 & 10979 & - & 10221 & -2.9 & +4.3 & -5.6 \\
\hline P6 & 21124 & 12412 & 17500 & - & 12422 & -41.2 & No gain & -41.2 \\
\hline \multicolumn{6}{|c|}{ Average reduction (-ve values only) without fibers (A1,A3,A5) } & -1.3 & & \\
\hline \multicolumn{6}{|c|}{ Average change (+ve and -ve values) without fibers $(\mathrm{A} 1, \mathrm{~A} 3, \mathrm{~A} 5)$} & -1.3 & +7.2 & -3.8 \\
\hline \multicolumn{6}{|c|}{ Average reduction (-ve values only) with fibers (A2,A4,A6) } & -37.7 & & \\
\hline \multicolumn{6}{|c|}{ Average change (+ve and -ve values) with fibers $(\mathrm{A} 2, \mathrm{~A} 4, \mathrm{~A} 6)$} & -37.7 & No gain & -41.7 \\
\hline
\end{tabular}

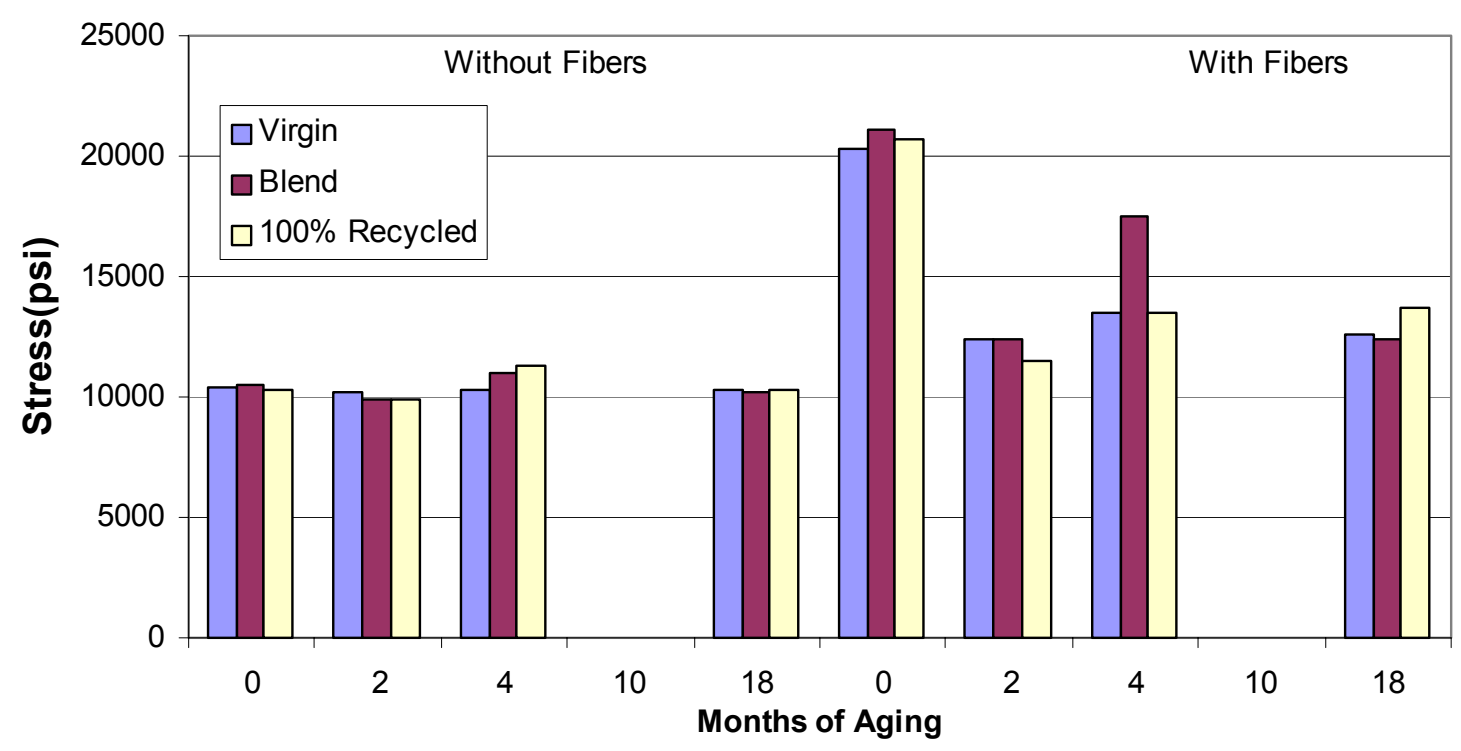

Note: Some intermittent results betw een 0 and 18 months may not be present due to no testing during that period

Figure F.11 Compressive Strength in Aged PC Specimens 
Table F.12 Compressive Stiffness Variations in Aged PC Specimens

\begin{tabular}{|c|c|c|c|c|c|c|c|c|}
\hline \multirow{3}{*}{\begin{tabular}{|c} 
Specimen \\
Type
\end{tabular}} & \multicolumn{5}{|c|}{ Compressive Stiffness (psi) } & \multicolumn{3}{|c|}{ \% Change } \\
\hline & \multicolumn{5}{|c|}{ Months of aging } & \multirow{2}{*}{$\begin{array}{l}\text { End of } \\
\text { period }\end{array}$} & \multirow{2}{*}{$\begin{array}{l}\text { Max gain } \\
\text { in period }\end{array}$} & \multirow{2}{*}{$\begin{array}{l}\text { Max red } \\
\text { in period }\end{array}$} \\
\hline & $\mathbf{0}$ & 2 & 4 & 10 & 18 & & & \\
\hline P1 & 0.21 & - & 0.283 & - & 0.320 & +52.4 & +52.4 & No red. \\
\hline P2 & 0.41 & - & 0.481 & - & 0.511 & +24.6 & +24.6 & No red. \\
\hline P3 & 0.20 & - & 0.302 & - & 0.344 & +72.0 & +72.0 & No red. \\
\hline P4 & 0.43 & - & 0.463 & - & 0.494 & +14.9 & +14.9 & No red. \\
\hline P5 & 0.21 & - & 0.355 & - & - & +69.0 & +69.0 & No red. \\
\hline P6 & 0.43 & - & 0.481 & - & 0.477 & +10.9 & +11.9 & No red. \\
\hline \multicolumn{6}{|c|}{ Average reduction (-ve values only) without fibers (A1,A3,A5) } & 0 & & \\
\hline \multicolumn{6}{|c|}{ Average change (+ve and -ve values) without fibers (A1,A3,A5) } & +64.5 & +64.5 & No red. \\
\hline \multicolumn{6}{|c|}{ Average reduction (-ve values only) with fibers (A2,A4,A6) } & 0 & & \\
\hline \multicolumn{6}{|c|}{ Average change (+ve and -ve values) with fibers $(\mathrm{A} 2, \mathrm{~A} 4, \mathrm{~A} 6)$} & +16.8 & +17.1 & No red. \\
\hline
\end{tabular}

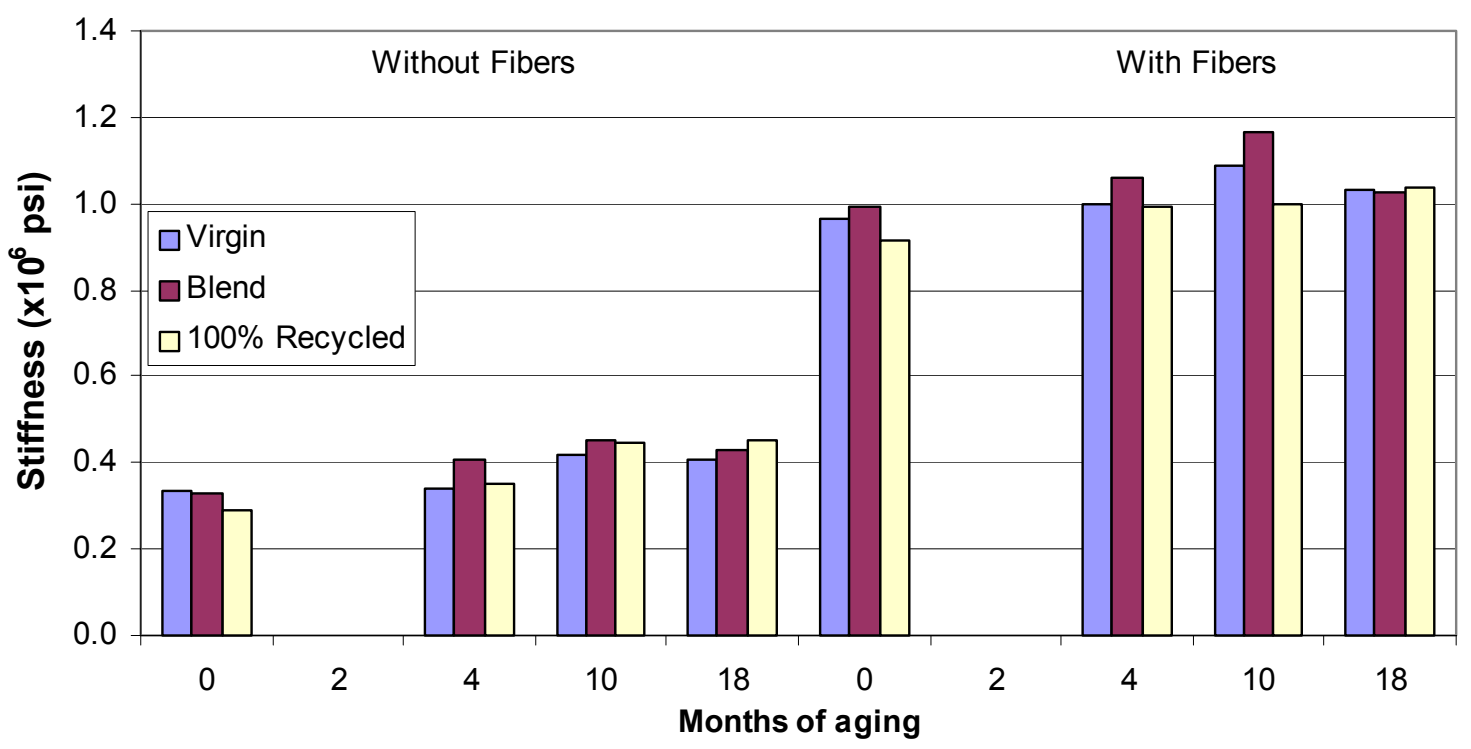

Note: Some intermittent results betw een 0 and 18 months may not be present due to no testing during that period

Figure F.12 Compressive Stiffness in Aged PC Specimens 
Table F.13 Impact Strength Variations in Aged ABS Specimens

\begin{tabular}{|c|c|c|c|c|c|c|c|c|}
\hline \multirow{3}{*}{$\begin{array}{c}\text { Specimen } \\
\text { Type }\end{array}$} & \multicolumn{5}{|c|}{ Impact Strength $\left(\right.$ ft-lbs $\left._{f}\right)$} & \multicolumn{3}{|c|}{ \% Change } \\
\hline & \multicolumn{5}{|c|}{ Months of aging } & \multirow{2}{*}{$\begin{array}{l}\text { End of } \\
\text { period }\end{array}$} & \multirow{2}{*}{\begin{tabular}{|l|} 
Max gain \\
in period
\end{tabular}} & \multirow{2}{*}{\begin{tabular}{|l} 
Max red \\
in perioc
\end{tabular}} \\
\hline & $\mathbf{0}$ & 2 & 4 & $\mathbf{1 0}$ & 18 & & & \\
\hline $\mathrm{A} 1$ & 3.49 & 3.67 & 3.76 & 3.44 & 3.41 & -2.3 & +7.8 & -2.3 \\
\hline A2 & 1.58 & 1.31 & 1.40 & 1.32 & 1.24 & -21.5 & No gain & -21.5 \\
\hline A3 & 2.17 & 1.91 & 1.24 & 0.83 & 0.87 & -59.7 & No gain & -61.6 \\
\hline A4 & 0.96 & 0.90 & 0.83 & 0.75 & 0.75 & -21.7 & No gain & -21.7 \\
\hline A5 & 2.38 & 2.86 & 2.54 & 1.77 & 1.46 & -38.8 & +20.3 & -38.8 \\
\hline A6 & 1.20 & 1.04 & 1.08 & 1.00 & 0.87 & -27.2 & No gain & -27.2 \\
\hline \multicolumn{6}{|c|}{ Average reduction (-ve values only) without fibers (A1,A3,A5) } & -33.6 & & \\
\hline \multicolumn{6}{|c|}{ Average change (+ve and -ve values) without fibers $(\mathrm{A} 1, \mathrm{~A} 3, \mathrm{~A} 5)$} & -33.6 & +14.1 & -34.2 \\
\hline \multicolumn{6}{|c|}{ Average reduction (-ve values only) with fibers (A2,A4,A6) } & -23.5 & & \\
\hline \multicolumn{6}{|c|}{ Average change (+ve and -ve values) with fibers $(\mathrm{A} 2, \mathrm{~A} 4, \mathrm{~A} 6)$} & -23.5 & No gain & -23.5 \\
\hline
\end{tabular}

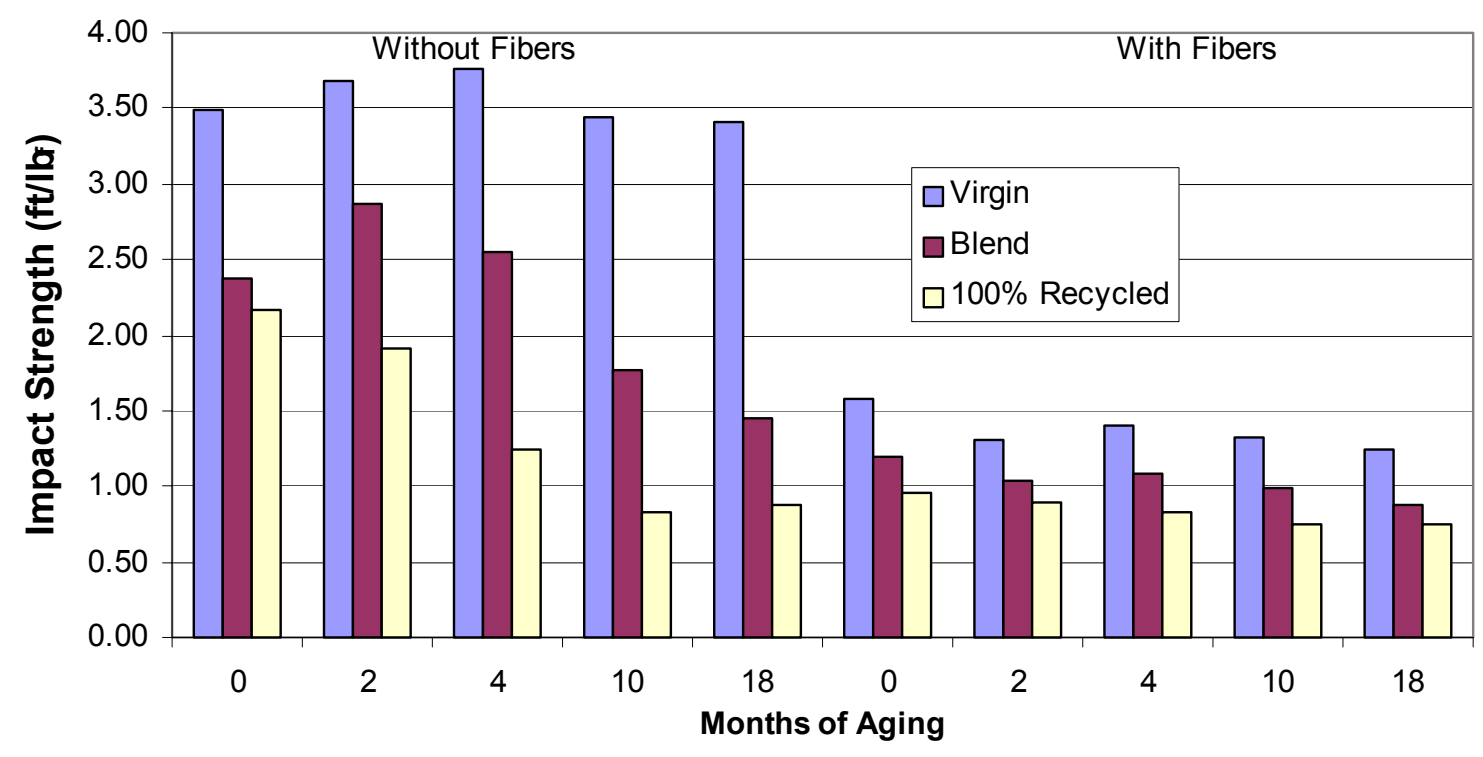

Figure F.13 Impact Strength in Aged ABS Specimens 
Table F.14 Impact Strength Variations in Aged PC Specimens

\begin{tabular}{|c|c|c|c|c|c|c|c|c|}
\hline \multirow{3}{*}{$\begin{array}{c}\text { Specimen } \\
\text { Type }\end{array}$} & \multicolumn{5}{|c|}{ Impact Strength $\left(\right.$ ft-lbs $\left._{\mathrm{f}}\right)$} & \multicolumn{3}{|c|}{$\%$ Change } \\
\hline & \multicolumn{5}{|c|}{ Months of aging } & \multirow{2}{*}{$\begin{array}{l}\text { End of } \\
\text { period }\end{array}$} & \multirow{2}{*}{$\begin{array}{l}\text { Max gain } \\
\text { in period }\end{array}$} & \multirow{2}{*}{$\begin{array}{l}\text { Max red } \\
\text { in period }\end{array}$} \\
\hline & $\mathbf{0}$ & 2 & 4 & 10 & 18 & & & \\
\hline P1 & 14.15 & - & 14.67 & 14.59 & 15.56 & +10.0 & +10.0 & No red. \\
\hline $\mathrm{P} 2$ & 3.01 & - & 1.48 & 1.40 & 1.48 & -50.7 & No gain & -53.4 \\
\hline P3 & 13.53 & - & 13.77 & 15.65 & 14.99 & +10.8 & +15.6 & No red. \\
\hline P4 & 1.81 & - & 0.91 & 0.91 & 0.79 & -56.2 & No gain & -56.2 \\
\hline P5 & 14.80 & - & 14.58 & 14.91 & 15.65 & +5.7 & +5.7 & -1.5 \\
\hline P6 & 2.67 & - & 1.24 & 1.32 & 1.20 & -55.1 & No gain & -55.1 \\
\hline \multicolumn{6}{|c|}{ Average reduction (-ve values only) without fibers $(\mathrm{A} 1, \mathrm{~A} 3, \mathrm{~A} 5)$} & 0 & & \\
\hline \multicolumn{6}{|c|}{ Average change (+ve and -ve values) without fibers $(\mathrm{A} 1, \mathrm{~A} 3, \mathrm{~A} 5)$} & +8.8 & +10.4 & -1.5 \\
\hline \multicolumn{6}{|c|}{ Average reduction (-ve values only) with fibers (A2,A4,A6) } & -54.0 & & \\
\hline \multicolumn{6}{|c|}{ Average change (+ve and -ve values) with fibers $(\mathrm{A} 2, \mathrm{~A} 4, \mathrm{~A} 6)$} & -54.0 & No gain & -54.9 \\
\hline
\end{tabular}

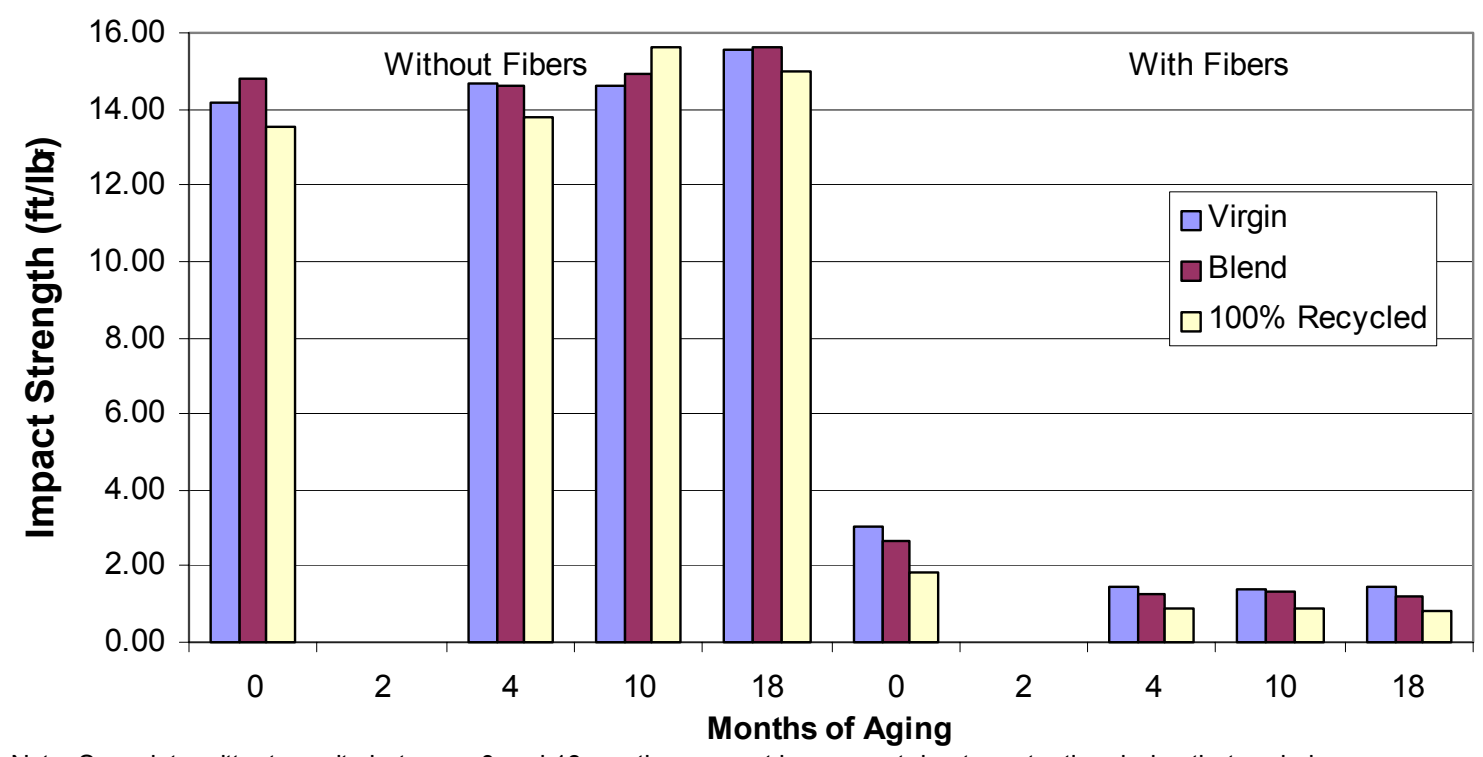

Note: Some intermittent results betw een 0 and 18 months may not be present due to no testing during that period

Figure F.14 Impact Strength in Aged PC Specimens 
Table F.15 Hardness Index Variations in Aged ABS Specimens

\begin{tabular}{|c|c|c|c|c|c|c|c|c|}
\hline \multirow{3}{*}{\begin{tabular}{|c} 
Specimen \\
Type
\end{tabular}} & \multicolumn{5}{|c|}{ Hardness Index } & \multicolumn{3}{|c|}{ \% Change } \\
\hline & \multicolumn{5}{|c|}{ Months of aging } & \multirow{2}{*}{$\begin{array}{l}\text { End of } \\
\text { period }\end{array}$} & \multirow{2}{*}{$\begin{array}{l}\text { Max gain } \\
\text { in period }\end{array}$} & \multirow{2}{*}{\begin{tabular}{|l} 
Max red. \\
in period
\end{tabular}} \\
\hline & $\mathbf{0}$ & 2 & 4 & 10 & 18 & & & \\
\hline A1 & 10.90 & 12.44 & 11.70 & 12.10 & 12.00 & +10.1 & +14.1 & No red. \\
\hline A2 & 11.30 & 12.80 & 11.50 & 11.00 & 10.90 & -3.5 & +13.3 & -3.5 \\
\hline A3 & 11.10 & 12.58 & 11.40 & 11.10 & 11.00 & -0.9 & +13.3 & -0.9 \\
\hline A4 & 11.20 & 11.76 & 11.30 & 10.80 & 10.60 & -5.4 & +5.0 & -5.4 \\
\hline A5 & 11.20 & 12.44 & 10.80 & 11.10 & 10.90 & -2.7 & +11.1 & -3.6 \\
\hline A6 & 11.20 & 12.16 & 11.00 & 10.90 & 10.80 & -3.6 & +8.6 & -3.6 \\
\hline \multicolumn{6}{|c|}{ Average reduction (-ve values only) without fibers (A1,A3,A5) } & -1.8 & & \\
\hline \multicolumn{6}{|c|}{ Average change (+ve and -ve values) without fibers (A1,A3,A5) } & +2.2 & +12.8 & -2.2 \\
\hline \multicolumn{6}{|c|}{ Average reduction (-ve values only) with fibers $(\mathrm{A} 2, \mathrm{~A} 4, \mathrm{~A} 6)$} & -4.2 & & \\
\hline \multicolumn{6}{|c|}{ Average change (+ve and -ve values) with fibers $(\mathrm{A} 2, \mathrm{~A} 4, \mathrm{~A} 6)$} & -4.2 & +8.9 & -4.2 \\
\hline
\end{tabular}

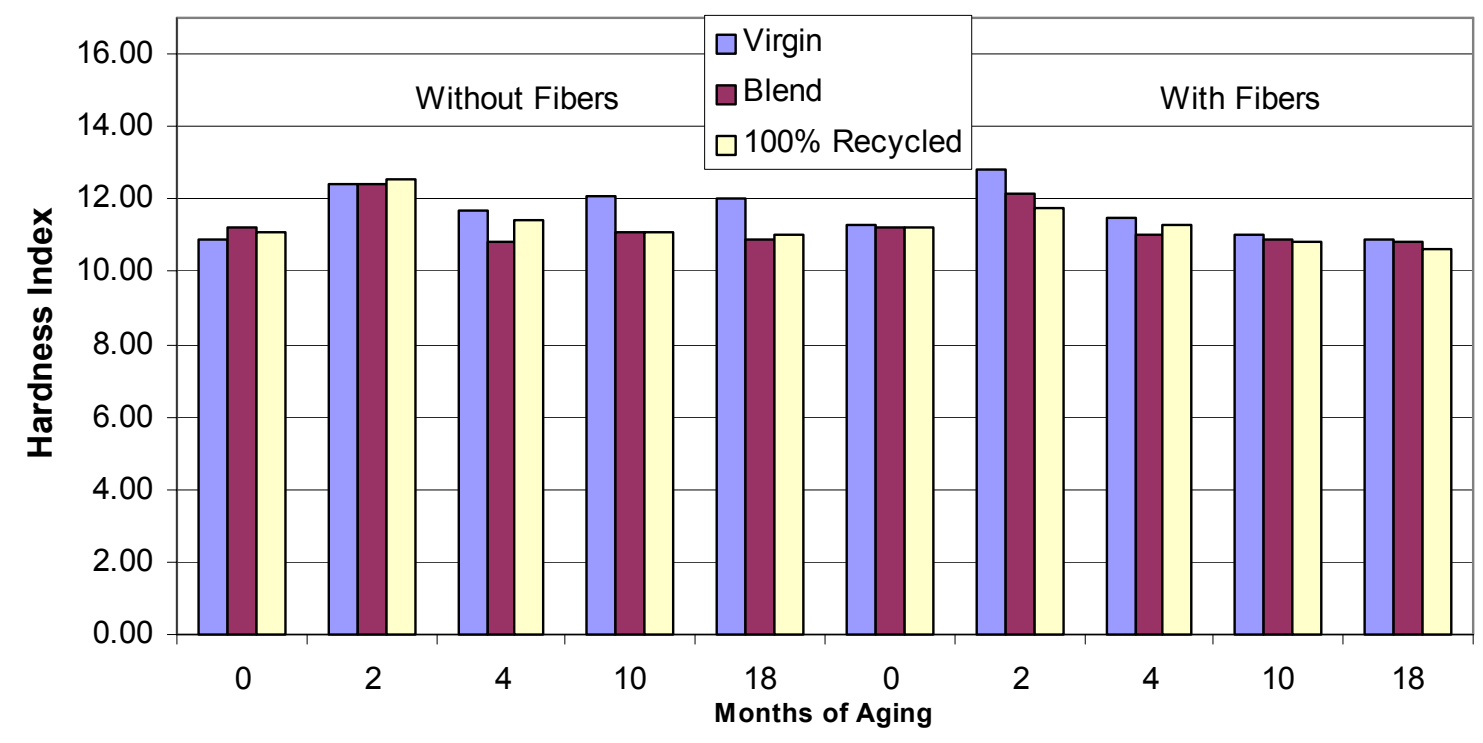

Figure F.15 Hardness Index in Aged ABS Specimens 
Table F.16 Hardness Index Variations in Aged PC Specimens

\begin{tabular}{|c|c|c|c|c|c|c|c|c|}
\hline \multirow{3}{*}{$\begin{array}{c}\text { Specimen } \\
\text { Type }\end{array}$} & \multicolumn{5}{|c|}{ Hardness Index } & \multicolumn{3}{|c|}{ \% Change } \\
\hline & \multicolumn{5}{|c|}{ Months of aging } & \multirow{2}{*}{$\begin{array}{l}\text { End of } \\
\text { period }\end{array}$} & \multirow{2}{*}{$\begin{array}{l}\text { Max gain } \\
\text { in period }\end{array}$} & \multirow{2}{*}{$\begin{array}{l}\text { Max red } \\
\text { in period }\end{array}$} \\
\hline & $\mathbf{0}$ & 2 & 4 & 10 & 18 & & & \\
\hline P1 & 10.60 & 12.00 & 11.30 & 11.10 & 11.00 & +3.8 & +13.2 & No red. \\
\hline $\mathrm{P} 2$ & 10.70 & 11.10 & 11.20 & 11.30 & 11.30 & +5.6 & +5.6 & No red. \\
\hline P3 & 10.60 & 11.50 & 10.80 & 11.10 & 11.00 & +3.8 & +8.5 & No red. \\
\hline P4 & 10.70 & 11.44 & 10.80 & 10.90 & 10.80 & +0.9 & +6.9 & No red. \\
\hline P5 & 10.70 & 10.74 & 11.20 & 10.90 & 10.90 & +1.9 & +4.7 & No red. \\
\hline P6 & 10.70 & 11.38 & 11.40 & 10.90 & 10.80 & +0.9 & +6.5 & No red. \\
\hline \multicolumn{6}{|c|}{ Average reduction (-ve values only) without fibers $(\mathrm{A} 1, \mathrm{~A} 3, \mathrm{~A} 5)$} & 0 & & \\
\hline \multicolumn{6}{|c|}{ Average change (+ve and -ve values) without fibers $(\mathrm{A} 1, \mathrm{~A} 3, \mathrm{~A} 5)$} & +3.1 & +8.8 & No red. \\
\hline \multicolumn{6}{|c|}{ Average reduction (-ve values only) with fibers (A2,A4,A6) } & 0 & & \\
\hline \multicolumn{6}{|c|}{ Average change (+ve and $-\mathrm{ve}$ values) with fibers $(\mathrm{A} 2, \mathrm{~A} 4, \mathrm{~A} 6)$} & +2.5 & +6.4 & No red. \\
\hline
\end{tabular}

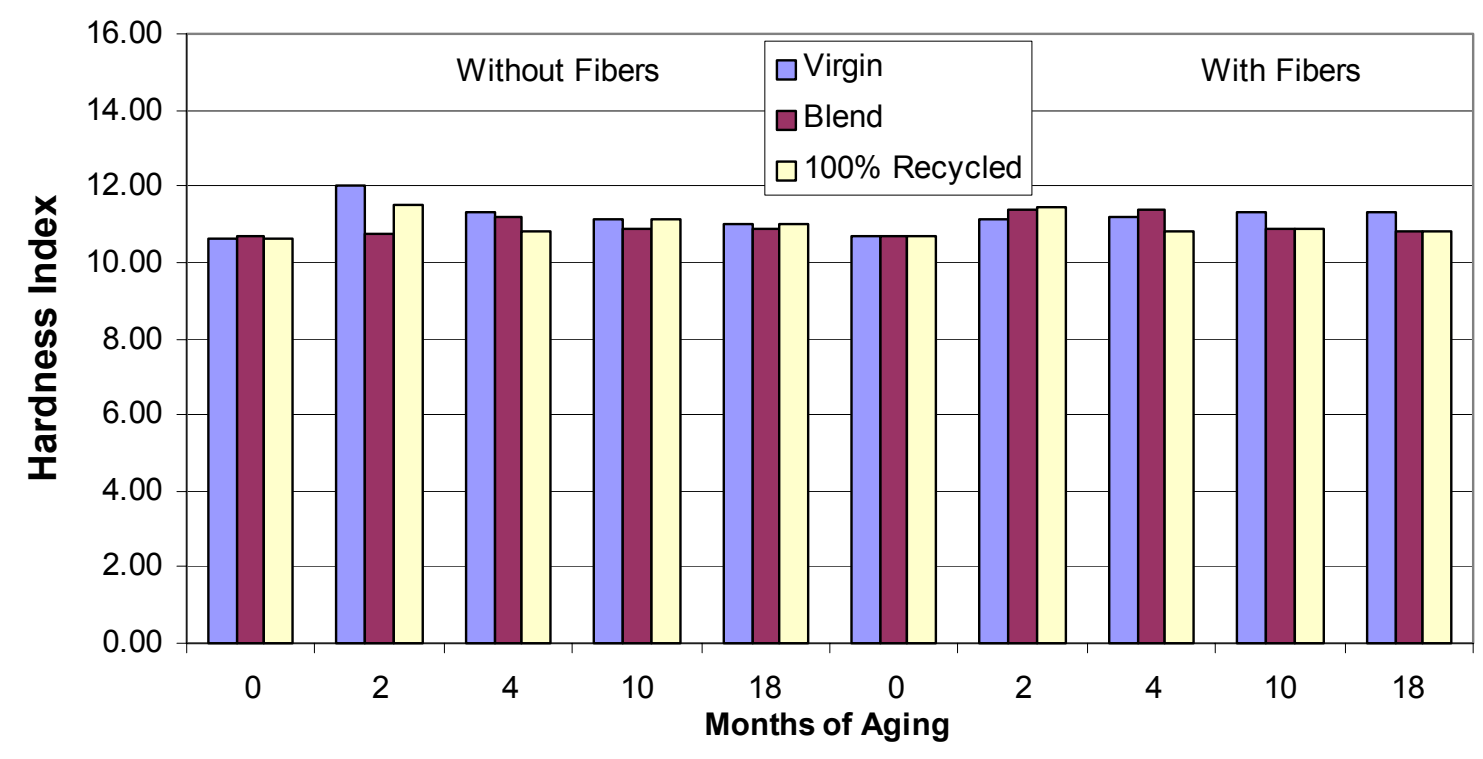

Figure F.16 Hardness Index in Aged PC Specimens 


\section{APPENDIX G}

\section{CREEP TEST RESULTS}

Table G.1 Creep Test Results for 100\% Recycled and Blend ABS without Fibers at 20\% Sustained Load

\begin{tabular}{|c|c|c|c|c|c|}
\hline Date & \multirow{2}{*}{ Days } & \multicolumn{4}{|c|}{ Specimens } \\
\cline { 3 - 6 } & & Strain Reading $(\mu \mathbf{s})$ & \multicolumn{2}{c|}{ Creep Coefficient } \\
\cline { 3 - 6 } & & $\mathrm{A} 3$ & $\mathrm{~A} 5$ & $\mathrm{~A} 3$ & $\mathrm{~A} 5$ \\
\hline $01 / 31 / 2000$ & 0 & 4116 & 3130 & 0.000 & 0.000 \\
\hline $02 / 01 / 2000$ & 1 & 4187 & 3313 & 0.017 & 0.058 \\
\hline $02 / 02 / 2000$ & 2 & 4242 & 3374 & 0.031 & 0.078 \\
\hline $02 / 03 / 2000$ & 3 & 4251 & 3385 & 0.033 & 0.081 \\
\hline $02 / 04 / 2000$ & 4 & 4384 & 3530 & 0.065 & 0.128 \\
\hline $02 / 05 / 2000$ & 5 & 4372 & 3528 & 0.062 & 0.127 \\
\hline $02 / 06 / 2000$ & 6 & 4391 & 3544 & 0.067 & 0.132 \\
\hline $02 / 07 / 2000$ & 7 & 4426 & 3586 & 0.075 & 0.146 \\
\hline $02 / 08 / 2000$ & 8 & 4451 & 3610 & 0.081 & 0.153 \\
\hline $02 / 09 / 2000$ & 9 & 4511 & 3667 & 0.096 & 0.172 \\
\hline $02 / 10 / 2000$ & 10 & 4550 & 3709 & 0.105 & 0.185 \\
\hline $02 / 11 / 2000$ & 11 & 4654 & 3810 & 0.131 & 0.217 \\
\hline $02 / 12 / 2000$ & 12 & 4620 & 3774 & 0.122 & 0.206 \\
\hline $02 / 13 / 2000$ & 13 & 4645 & 3805 & 0.129 & 0.216 \\
\hline $02 / 14 / 2000$ & 14 & 4761 & 3927 & 0.157 & 0.255 \\
\hline $02 / 15 / 2000$ & 15 & 4758 & 3928 & 0.156 & 0.255 \\
\hline $02 / 16 / 2000$ & 16 & 4782 & 3945 & 0.162 & 0.260 \\
\hline $02 / 17 / 2000$ & 17 & 4885 & 4085 & 0.187 & 0.305 \\
\hline $02 / 18 / 2000$ & 18 & 4874 & 4035 & 0.184 & 0.289 \\
\hline $02 / 19 / 2000$ & 19 & 4836 & 4006 & 0.175 & 0.280 \\
\hline $02 / 20 / 2000$ & 20 & 4837 & 4002 & 0.175 & 0.279 \\
\hline $02 / 21 / 2000$ & 21 & 4871 & 4045 & 0.183 & 0.292 \\
\hline $02 / 22 / 2000$ & 22 & 4965 & 4145 & 0.206 & 0.324 \\
\hline $02 / 23 / 2000$ & 23 & 5090 & 4276 & 0.237 & 0.366 \\
\hline $02 / 24 / 2000$ & 24 & 5182 & 4345 & 0.259 & 0.388 \\
\hline $02 / 25 / 2000$ & 25 & 5279 & 4431 & 0.283 & 0.416 \\
\hline $02 / 26 / 2000$ & 26 & 5332 & 4502 & 0.295 & 0.438 \\
\hline $02 / 27 / 2000$ & 27 & 5370 & 4536 & 0.305 & 0.449 \\
\hline $02 / 28 / 2000$ & 28 & 5335 & 4513 & 0.296 & 0.442 \\
\hline $02 / 29 / 2000$ & 29 & 5305 & 4478 & 0.289 & 0.431 \\
\hline $03 / 01 / 2000$ & 30 & 5346 & 4503 & 0.299 & 0.439 \\
\hline $03 / 02 / 2000$ & 31 & 5305 & 4470 & 0.289 & 0.428 \\
\hline $03 / 03 / 2000$ & 32 & 5305 & 4469 & 0.289 & 0.428 \\
\hline $03 / 04 / 2000$ & 33 & 5301 & 4465 & 0.288 & 0.427 \\
\hline $03 / 05 / 2000$ & 34 & 5302 & 4472 & 0.288 & 0.429 \\
\hline $03 / 06 / 2000$ & 35 & 5350 & 4529 & 0.300 & 0.447 \\
\hline
\end{tabular}




\begin{tabular}{|l|l|l|l|l|l|}
\hline $03 / 07 / 2000$ & 36 & 5432 & 4609 & 0.320 & 0.473 \\
\hline $03 / 08 / 2000$ & 37 & 5630 & 4816 & 0.368 & 0.539 \\
\hline $03 / 09 / 2000$ & 38 & 5684 & 4864 & 0.381 & 0.554 \\
\hline $03 / 10 / 2000$ & 39 & 5520 & 4706 & 0.341 & 0.504 \\
\hline $03 / 11 / 2000$ & 40 & 5555 & 4766 & 0.350 & 0.523 \\
\hline $03 / 12 / 2000$ & 41 & 5480 & 4630 & 0.331 & 0.479 \\
\hline $03 / 13 / 2000$ & 42 & 5470 & 4645 & 0.329 & 0.484 \\
\hline $03 / 14 / 2000$ & 43 & 5465 & 4639 & 0.328 & 0.482 \\
\hline $03 / 15 / 2000$ & 44 & 5539 & 4718 & 0.346 & 0.507 \\
\hline $03 / 16 / 2000$ & 45 & 5592 & 4773 & 0.359 & 0.525 \\
\hline $03 / 17 / 2000$ & 46 & 5491 & 4682 & 0.334 & 0.496 \\
\hline $03 / 18 / 2000$ & 47 & 5470 & 4651 & 0.329 & 0.486 \\
\hline $03 / 19 / 2000$ & 48 & 5478 & 4667 & 0.331 & 0.491 \\
\hline $03 / 20 / 2000$ & 49 & 5516 & 4705 & 0.340 & 0.503 \\
\hline $03 / 21 / 2000$ & 50 & 5562 & 4739 & 0.351 & 0.514 \\
\hline $03 / 22 / 2000$ & 51 & 5670 & 4845 & 0.378 & 0.548 \\
\hline $03 / 23 / 2000$ & 52 & 5773 & 4955 & 0.403 & 0.583 \\
\hline $03 / 24 / 2000$ & 53 & 5863 & 5052 & 0.424 & 0.614 \\
\hline $03 / 25 / 2000$ & 54 & 5830 & 5020 & 0.416 & 0.604 \\
\hline $03 / 26 / 2000$ & 55 & 5743 & 4945 & 0.395 & 0.580 \\
\hline $03 / 27 / 2000$ & 56 & 5752 & 4950 & 0.397 & 0.581 \\
\hline $03 / 29 / 2000$ & 58 & 5700 & 4901 & 0.385 & 0.566 \\
\hline $03 / 30 / 2000$ & 59 & 5694 & 4890 & 0.383 & 0.562 \\
\hline $03 / 31 / 2000$ & 60 & 5690 & 4885 & 0.382 & 0.561 \\
\hline $04 / 03 / 2000$ & 63 & 5978 & 5175 & 0.452 & 0.653 \\
\hline $04 / 05 / 2000$ & 65 & 5896 & 5080 & 0.432 & 0.623 \\
\hline $04 / 07 / 2000$ & 67 & 5993 & 5168 & 0.456 & 0.651 \\
\hline $04 / 10 / 2000$ & 70 & 5920 & 5107 & 0.438 & 0.632 \\
\hline $04 / 11 / 2000$ & 71 & 5902 & 5090 & 0.434 & 0.626 \\
\hline $04 / 13 / 2000$ & 73 & 5971 & 5195 & 0.451 & 0.660 \\
\hline $04 / 14 / 2000$ & 74 & 6034 & 5231 & 0.466 & 0.671 \\
\hline $04 / 17 / 2000$ & 77 & 6082 & 5262 & 0.478 & 0.681 \\
\hline $04 / 18 / 2000$ & 78 & 6117 & 5295 & 0.486 & 0.692 \\
\hline $04 / 20 / 2000$ & 80 & 6363 & 5574 & 0.546 & 0.781 \\
\hline $04 / 21 / 2000$ & 81 & 6432 & 5626 & 0.563 & 0.797 \\
\hline $04 / 24 / 2000$ & 84 & 6224 & 5407 & 0.512 & 0.727 \\
\hline $04 / 26 / 2000$ & 86 & 6217 & 5412 & 0.510 & 0.729 \\
\hline $04 / 28 / 2000$ & 88 & 6256 & 5454 & 0.520 & 0.742 \\
\hline $05 / 01 / 2000$ & 91 & 6365 & 5565 & 0.546 & 0.778 \\
\hline $05 / 03 / 2000$ & 93 & 6453 & 5640 & 0.568 & 0.802 \\
\hline $05 / 05 / 2000$ & 95 & 6755 & 5946 & 0.641 & 0.900 \\
\hline $05 / 08 / 2000$ & 98 & 7072 & 6253 & 0.718 & 0.998 \\
\hline $05 / 10 / 2000$ & 100 & 6980 & 6130 & 0.696 & 0.958 \\
\hline $05 / 12 / 2000$ & 102 & 7089 & 6251 & 0.722 & 0.997 \\
\hline $05 / 15 / 2000$ & 105 & 6800 & 5983 & 0.652 & 0.912 \\
\hline $05 / 17 / 2000$ & 107 & 6955 & 6122 & 0.690 & 0.956 \\
\hline $05 / 19 / 2000$ & 109 & 7009 & 6140 & 0.703 & 0.962 \\
\hline $05 / 22 / 2000$ & 112 & 7030 & 6150 & 0.708 & 0.965 \\
\hline
\end{tabular}




\begin{tabular}{|l|c|c|c|c|c|}
\hline $05 / 26 / 2000$ & 116 & 7193 & 6316 & 0.748 & 1.018 \\
\hline $05 / 30 / 2000$ & 120 & 7076 & 6201 & 0.719 & 0.981 \\
\hline $06 / 01 / 2000$ & 122 & 7579 & 6709 & 0.841 & 1.143 \\
\hline $06 / 02 / 2000$ & 123 & 7720 & 6845 & 0.876 & 1.187 \\
\hline $06 / 05 / 2000$ & 126 & 7497 & 6636 & 0.821 & 1.120 \\
\hline $06 / 07 / 2000$ & 128 & 7346 & 6450 & 0.785 & 1.061 \\
\hline $06 / 09 / 2000$ & 130 & 7665 & 6829 & 0.862 & 1.182 \\
\hline $06 / 12 / 2000$ & 133 & 7950 & 7089 & 0.931 & 1.265 \\
\hline $06 / 14 / 2000$ & 135 & 8247 & 7400 & 1.004 & 1.364 \\
\hline $06 / 16 / 2000$ & 137 & 8381 & 7492 & 1.036 & 1.394 \\
\hline $06 / 21 / 2000$ & 142 & 8350 & 7446 & 1.029 & 1.379 \\
\hline $06 / 23 / 2000$ & 144 & 8521 & 7625 & 1.070 & 1.436 \\
\hline $06 / 26 / 2000$ & 147 & 8539 & 7650 & 1.075 & 1.444 \\
\hline $06 / 28 / 2000$ & 149 & 7138 & 6195 & 0.734 & 0.979 \\
\hline $07 / 06 / 2000$ & 157 & 7318 & 6382 & 0.778 & 1.039 \\
\hline $07 / 12 / 2000$ & 163 & 7500 & 6559 & 0.822 & 1.096 \\
\hline $07 / 17 / 2000$ & 168 & 7194 & 6223 & 0.748 & 0.988 \\
\hline $07 / 22 / 2000$ & 173 & 7650 & 6673 & 0.859 & 1.132 \\
\hline $07 / 27 / 2000$ & 178 & 8062 & 7142 & 0.959 & 1.282 \\
\hline $07 / 31 / 2000$ & 182 & 7642 & 6680 & 0.857 & 1.134 \\
\hline $08 / 04 / 2000$ & 186 & 7894 & 6942 & 0.918 & 1.218 \\
\hline $08 / 09 / 2000$ & 191 & 7839 & 6900 & 0.905 & 1.204 \\
\hline $08 / 14 / 2000$ & 196 & 7491 & 6528 & 0.820 & 1.086 \\
\hline $08 / 19 / 2000$ & 201 & 7860 & 6935 & 0.910 & 1.216 \\
\hline $08 / 28 / 2000$ & 210 & 7949 & 6970 & 0.931 & 1.227 \\
\hline $09 / 06 / 2000$ & 219 & 7808 & 6783 & 0.897 & 1.167 \\
\hline $09 / 11 / 2000$ & 224 & 8365 & 7435 & 1.032 & 1.375 \\
\hline $09 / 15 / 2000$ & 228 & 7994 & 6999 & 0.942 & 1.236 \\
\hline $09 / 18 / 2000$ & 231 & 8035 & 7029 & 0.952 & 1.246 \\
\hline $09 / 22 / 2000$ & 235 & 8015 & 6997 & 0.947 & 1.235 \\
\hline $09 / 25 / 2000$ & 238 & 7928 & 6917 & 0.926 & 1.210 \\
\hline $10 / 02 / 2000$ & 245 & 8010 & 7011 & 0.946 & 1.240 \\
\hline $10 / 05 / 2000$ & 248 & 8452 & 7437 & 1.053 & 1.376 \\
\hline $10 / 08 / 2000$ & 251 & 8821 & 7816 & 1.143 & 1.497 \\
\hline $10 / 13 / 2000$ & 256 & 8923 & 8199 & 1.168 & 1.619 \\
\hline $10 / 18 / 2000$ & 261 & 8965 & 8456 & 1.178 & 1.702 \\
\hline $02 / 08 / 2001$ & 374 & 9256 & 8056 & 1.249 & 1.574 \\
\hline $05 / 15 / 2001$ & 470 & 9462 & 8365 & 1.299 & 1.673 \\
\hline $07 / 12 / 2001$ & 528 & 9863 & 8562 & 1.396 & 1.735 \\
\hline $10 / 17 / 2001$ & 625 & 10125 & 8921 & 1.460 & 1.850 \\
\hline $01 / 16 / 2002$ & 716 & 10245 & 8912 & 1.489 & 1.847 \\
\hline $04 / 12 / 2002$ & 802 & 10598 & 9105 & 1.575 & 1.909 \\
\hline $06 / 04 / 2002$ & 855 & 10965 & 9532 & 1.664 & 2.045 \\
\hline $08 / 12 / 2002$ & 924 & 11245 & 12658 & 1.732 & 2.075 \\
\hline
\end{tabular}


Table G.2 Creep Test Results for Virgin ABS without Fibers; Virgin, Blend, and 100\%

Recycled with Fibers at $20 \%$ Sustained Load

\begin{tabular}{|c|c|c|c|c|c|c|c|c|c|}
\hline \multirow[t]{3}{*}{ Date } & \multirow[t]{3}{*}{ Days } & \multicolumn{8}{|c|}{ Specimens } \\
\hline & & \multicolumn{4}{|c|}{ Strain Reading $(\mu \mathrm{s})$} & \multicolumn{4}{|c|}{ Creep Coefficient } \\
\hline & & A1 & A2 & A4 & A6 & A1 & A2 & A4 & A6 \\
\hline $02 / 28 / 2000$ & 0 & 0 & 0 & & 0 & & & & \\
\hline 02/29/2000 & 1 & 3424 & 2839 & 0 & 2431 & 0.000 & 0.000 & & 0.000 \\
\hline $03 / 01 / 2000$ & 2 & 3472 & 2856 & 3451 & 2442 & 0.014 & 0.006 & 0.000 & 0.005 \\
\hline 03/02/2000 & 3 & 3549 & 2874 & 3482 & 2467 & 0.037 & 0.012 & 0.009 & 0.015 \\
\hline 03/03/2000 & 4 & 3568 & 2886 & 3504 & 2456 & 0.042 & 0.017 & 0.015 & 0.010 \\
\hline $03 / 04 / 2000$ & 5 & 3592 & 2901 & 3505 & 2465 & 0.049 & 0.022 & 0.016 & 0.014 \\
\hline 03/05/2000 & 6 & 3660 & 2918 & 3526 & 2479 & 0.069 & 0.028 & 0.022 & 0.020 \\
\hline 03/06/2000 & 7 & 3718 & 2928 & 3540 & 2495 & 0.086 & 0.031 & 0.026 & 0.026 \\
\hline 03/07/2000 & 8 & 3816 & 2941 & 3568 & 2521 & 0.114 & 0.036 & 0.034 & 0.037 \\
\hline $03 / 08 / 2000$ & 9 & 4027 & 2990 & 3619 & 2579 & 0.176 & 0.053 & 0.049 & 0.061 \\
\hline 03/09/2000 & 10 & 4126 & 3007 & 3649 & 2601 & 0.205 & 0.059 & 0.057 & 0.070 \\
\hline $03 / 10 / 2000$ & 11 & 4002 & 2993 & 3620 & 2564 & 0.169 & 0.054 & 0.049 & 0.055 \\
\hline $03 / 11 / 2000$ & 12 & 4008 & 2981 & 3627 & 2571 & 0.171 & 0.050 & 0.051 & 0.058 \\
\hline $03 / 12 / 2000$ & 13 & 3942 & 2976 & 3607 & 2547 & 0.151 & 0.048 & 0.045 & 0.048 \\
\hline $03 / 13 / 2000$ & 14 & 3942 & 2987 & 3607 & 2550 & 0.151 & 0.052 & 0.045 & 0.049 \\
\hline $03 / 14 / 2000$ & 15 & 3939 & 2987 & 3607 & 2553 & 0.150 & 0.052 & 0.045 & 0.050 \\
\hline $03 / 15 / 2000$ & 16 & 4014 & 2998 & 3629 & 2575 & 0.172 & 0.056 & 0.052 & 0.059 \\
\hline $03 / 16 / 2000$ & 17 & 4078 & 2955 & 3644 & 2594 & 0.191 & 0.041 & 0.056 & 0.067 \\
\hline $03 / 17 / 2000$ & 18 & 4019 & 3008 & 3631 & 2572 & 0.174 & 0.060 & 0.052 & 0.058 \\
\hline $03 / 18 / 2000$ & 19 & 3975 & 3008 & 3623 & 2561 & 0.161 & 0.060 & 0.050 & 0.053 \\
\hline $03 / 19 / 2000$ & 20 & 3995 & 3000 & 3624 & 2568 & 0.167 & 0.057 & 0.050 & 0.056 \\
\hline $03 / 20 / 2000$ & 21 & 4049 & 2991 & 3635 & 2577 & 0.183 & 0.054 & 0.053 & 0.060 \\
\hline 03/21/2000 & 22 & 4065 & 2991 & 3645 & 2594 & 0.187 & 0.054 & 0.056 & 0.067 \\
\hline $03 / 22 / 2000$ & 23 & 4173 & 2996 & 3676 & 2617 & 0.219 & 0.055 & 0.065 & 0.077 \\
\hline $03 / 23 / 2000$ & 24 & 4289 & 3043 & 3702 & 2648 & 0.253 & 0.072 & 0.073 & 0.089 \\
\hline $03 / 24 / 2000$ & 25 & 4387 & 3033 & 3730 & 2675 & 0.281 & 0.068 & 0.081 & 0.100 \\
\hline $03 / 25 / 2000$ & 26 & 4383 & 3029 & 3730 & 2670 & 0.280 & 0.067 & 0.081 & 0.098 \\
\hline $03 / 26 / 2000$ & 27 & 4300 & 3000 & 3715 & 2651 & 0.256 & 0.057 & 0.076 & 0.090 \\
\hline $03 / 27 / 2000$ & 28 & 4302 & 3048 & 3713 & 2651 & 0.256 & 0.074 & 0.076 & 0.090 \\
\hline $03 / 29 / 2000$ & 30 & 4300 & 3063 & 3709 & 2650 & 0.256 & 0.079 & 0.075 & 0.090 \\
\hline 03/30/2000 & 31 & 4300 & 3078 & 3703 & 2651 & 0.256 & 0.084 & 0.073 & 0.090 \\
\hline $03 / 31 / 2000$ & 32 & 4300 & 3075 & 3702 & 2652 & 0.256 & 0.083 & 0.073 & 0.091 \\
\hline $04 / 03 / 2000$ & 35 & 4527 & 3100 & 3768 & 2715 & 0.322 & 0.092 & 0.092 & 0.117 \\
\hline $04 / 05 / 2000$ & 37 & 4475 & 3109 & 3760 & 2700 & 0.307 & 0.095 & 0.090 & 0.111 \\
\hline $04 / 07 / 2000$ & 39 & 4572 & 3045 & 3779 & 2716 & 0.335 & 0.073 & 0.095 & 0.117 \\
\hline $04 / 10 / 2000$ & 42 & 4501 & 3068 & 3772 & 2705 & 0.315 & 0.081 & 0.093 & 0.113 \\
\hline $04 / 11 / 2000$ & 43 & 4483 & 3064 & 3760 & 2702 & 0.309 & 0.079 & 0.090 & 0.111 \\
\hline $04 / 13 / 2000$ & 45 & 4563 & 3076 & 3774 & 2721 & 0.333 & 0.083 & 0.094 & 0.119 \\
\hline $04 / 14 / 2000$ & 46 & 4640 & 3085 & 3797 & 2734 & 0.355 & 0.087 & 0.100 & 0.125 \\
\hline $04 / 17 / 2000$ & 49 & 4680 & 3084 & 3824 & 2742 & 0.367 & 0.086 & 0.108 & 0.128 \\
\hline $04 / 18 / 2000$ & 50 & 4702 & 3084 & 3844 & 2770 & 0.373 & 0.086 & 0.114 & 0.139 \\
\hline $04 / 20 / 2000$ & 52 & 4990 & 3130 & 3895 & 2827 & 0.457 & 0.103 & 0.129 & 0.163 \\
\hline
\end{tabular}




\begin{tabular}{|c|c|c|c|c|c|c|c|c|c|}
\hline $04 / 21 / 2000$ & 53 & 5057 & 3131 & 3909 & 2846 & 0.477 & 0.103 & 0.133 & 0.171 \\
\hline $04 / 24 / 2000$ & 56 & 4831 & 3122 & 3861 & 2796 & 0.411 & 0.100 & 0.119 & 0.150 \\
\hline $04 / 26 / 2000$ & 58 & 4859 & 3199 & 3861 & 2798 & 0.419 & 0.127 & 0.119 & 0.151 \\
\hline $04 / 28 / 2000$ & 60 & 4901 & 3190 & 3867 & 2805 & 0.431 & 0.124 & 0.121 & 0.154 \\
\hline $05 / 01 / 2000$ & 63 & 5015 & 3203 & 3895 & 2830 & 0.465 & 0.128 & 0.129 & 0.164 \\
\hline $05 / 03 / 2000$ & 65 & 5116 & 3205 & 3915 & 2850 & 0.494 & 0.129 & 0.134 & 0.172 \\
\hline $05 / 05 / 2000$ & 67 & 5390 & 3214 & 3990 & 2930 & 0.574 & 0.132 & 0.156 & 0.205 \\
\hline $05 / 08 / 2000$ & 70 & 5730 & 3325 & 4083 & 3013 & 0.673 & 0.171 & 0.183 & 0.239 \\
\hline $05 / 10 / 2000$ & 72 & 5652 & 3297 & 4074 & 2983 & 0.651 & 0.161 & 0.181 & 0.227 \\
\hline $05 / 12 / 2000$ & 74 & 5732 & 3266 & 4081 & 3003 & 0.674 & 0.150 & 0.183 & 0.235 \\
\hline $05 / 15 / 2000$ & 77 & 5469 & 3312 & 4029 & 2953 & 0.597 & 0.167 & 0.167 & 0.215 \\
\hline $05 / 17 / 2000$ & 79 & 5596 & 3304 & 4056 & 2975 & 0.634 & 0.164 & 0.175 & 0.224 \\
\hline $05 / 19 / 2000$ & 81 & 5674 & 3300 & 4085 & 2978 & 0.657 & 0.162 & 0.184 & 0.225 \\
\hline $05 / 22 / 2000$ & 84 & 5750 & 3315 & 4106 & 3009 & 0.679 & 0.168 & 0.190 & 0.238 \\
\hline $05 / 26 / 2000$ & 88 & 5910 & 3376 & 4158 & 3048 & 0.726 & 0.189 & 0.205 & 0.254 \\
\hline $05 / 30 / 2000$ & 92 & 5964 & 3335 & 4140 & 3026 & 0.742 & 0.175 & 0.200 & 0.245 \\
\hline $06 / 01 / 2000$ & 94 & 6236 & 3409 & 4242 & 3140 & 0.821 & 0.201 & 0.229 & 0.292 \\
\hline $06 / 02 / 2000$ & 95 & 6379 & 3473 & 4273 & 3170 & 0.863 & 0.223 & 0.238 & 0.304 \\
\hline $06 / 05 / 2000$ & 98 & 6167 & 3423 & 4221 & 3116 & 0.801 & 0.206 & 0.223 & 0.282 \\
\hline $06 / 07 / 2000$ & 100 & 6047 & 3418 & 4197 & 3081 & 0.766 & 0.204 & 0.216 & 0.267 \\
\hline $06 / 09 / 2000$ & 102 & 6361 & 3484 & 4269 & 3160 & 0.858 & 0.227 & 0.237 & 0.300 \\
\hline $06 / 12 / 2000$ & 105 & 6609 & 3640 & 4287 & 3216 & 0.930 & 0.282 & 0.242 & 0.323 \\
\hline $06 / 14 / 2000$ & 107 & 6915 & 3730 & 4367 & 3282 & 1.020 & 0.314 & 0.265 & 0.350 \\
\hline $06 / 16 / 2000$ & 109 & 7217 & 3825 & 4437 & 3352 & 1.108 & 0.347 & 0.286 & 0.379 \\
\hline $06 / 21 / 2000$ & 114 & 7645 & 3705 & 4380 & 3323 & 1.233 & 0.305 & 0.269 & 0.367 \\
\hline $06 / 23 / 2000$ & 116 & 6999 & 3722 & 4365 & 3304 & 1.044 & 0.311 & 0.265 & 0.359 \\
\hline $06 / 26 / 2000$ & 119 & 7107 & 3781 & 4406 & 3346 & 1.076 & 0.332 & 0.277 & 0.376 \\
\hline $06 / 28 / 2000$ & 121 & 7107 & 3791 & 4395 & 3358 & 1.076 & 0.335 & 0.274 & 0.381 \\
\hline $07 / 06 / 2000$ & 129 & 7108 & 3367 & 4398 & 3342 & 1.076 & 0.186 & 0.274 & 0.375 \\
\hline $07 / 12 / 2000$ & 135 & 7216 & 3377 & 4423 & 3387 & 1.107 & 0.190 & 0.282 & 0.393 \\
\hline $07 / 17 / 2000$ & 140 & 6752 & 3733 & 4360 & 3325 & 0.972 & 0.315 & 0.263 & 0.368 \\
\hline $07 / 22 / 2000$ & 145 & 7437 & 3861 & 4481 & 3431 & 1.172 & 0.360 & 0.298 & 0.411 \\
\hline $07 / 27 / 2000$ & 150 & 7725 & 3936 & 4553 & 3493 & 1.256 & 0.386 & 0.319 & 0.437 \\
\hline $07 / 31 / 2000$ & 154 & 7408 & 3860 & 4477 & 3420 & 1.164 & 0.360 & 0.297 & 0.407 \\
\hline $08 / 04 / 2000$ & 158 & 7589 & 3368 & 4518 & 3437 & 1.216 & 0.186 & 0.309 & 0.414 \\
\hline $08 / 09 / 2000$ & 163 & 7559 & 3826 & 4504 & 3443 & 1.208 & 0.348 & 0.305 & 0.416 \\
\hline $08 / 14 / 2000$ & 168 & 7264 & 3679 & 4425 & 3371 & 1.121 & 0.296 & 0.282 & 0.387 \\
\hline $08 / 19 / 2000$ & 173 & 7560 & 3846 & 4500 & 3399 & 1.208 & 0.355 & 0.304 & 0.398 \\
\hline $08 / 28 / 2000$ & 182 & 7623 & 3820 & 4526 & 3468 & 1.226 & 0.346 & 0.312 & 0.427 \\
\hline $09 / 06 / 2000$ & 191 & 7469 & 3518 & 4471 & 3353 & 1.181 & 0.239 & 0.296 & 0.379 \\
\hline $09 / 11 / 2000$ & 196 & 7966 & 3891 & 4605 & 3539 & 1.327 & 0.371 & 0.334 & 0.456 \\
\hline $09 / 15 / 2000$ & 200 & 7588 & 3856 & 4511 & 3453 & 1.216 & 0.358 & 0.307 & 0.420 \\
\hline $09 / 18 / 2000$ & 203 & 7545 & 3854 & 4496 & 3419 & 1.204 & 0.358 & 0.303 & 0.406 \\
\hline $09 / 22 / 2000$ & 207 & 7538 & 3854 & 4492 & 3426 & 1.202 & 0.358 & 0.302 & 0.409 \\
\hline $09 / 25 / 2000$ & 210 & 7461 & 3806 & 4476 & 3348 & 1.179 & 0.341 & 0.297 & 0.377 \\
\hline $10 / 02 / 2000$ & 217 & 7531 & 3828 & 4484 & 3434 & 1.199 & 0.348 & 0.299 & 0.413 \\
\hline $10 / 05 / 2000$ & 220 & 7831 & 3908 & 4572 & 3516 & 1.287 & 0.377 & 0.325 & 0.446 \\
\hline $10 / 08 / 2000$ & 223 & 8025 & 4025 & 4605 & 3548 & 1.344 & 0.418 & 0.334 & 0.459 \\
\hline
\end{tabular}




\begin{tabular}{|l|l|l|l|l|l|l|r|r|r|}
\hline $10 / 13 / 2000$ & 228 & 8047 & 4054 & 4634 & 3583 & 1.350 & 0.428 & 0.343 & 0.474 \\
\hline $10 / 18 / 2000$ & 233 & 8089 & 4102 & 4656 & 3625 & 1.362 & 0.445 & 0.349 & 0.491 \\
\hline $02 / 08 / 2001$ & 346 & 8187 & 4385 & 4768 & 3864 & 1.391 & 0.545 & 0.382 & 0.589 \\
\hline $05 / 15 / 2001$ & 442 & 8274 & 4525 & 4852 & 4125 & 1.416 & 0.594 & 0.406 & 0.697 \\
\hline $07 / 12 / 2001$ & 500 & 8324 & 4795 & 5212 & 4287 & 1.431 & 0.689 & 0.510 & 0.763 \\
\hline $10 / 17 / 2001$ & 597 & 8402 & 5648 & 5487 & 4406 & 1.454 & 0.650 & 0.590 & 0.812 \\
\hline $01 / 16 / 2002$ & 688 & 8492 & 5864 & 5612 & 4585 & 1.480 & 0.713 & 0.626 & 0.886 \\
\hline $04 / 12 / 2002$ & 774 & 8570 & 5956 & 5741 & 4664 & 1.503 & 0.739 & 0.664 & 0.919 \\
\hline $06 / 04 / 2002$ & 827 & 8820 & 6104 & 5892 & 4731 & 1.576 & 0.783 & 0.707 & 0.946 \\
\hline $08 / 12 / 2002$ & 896 & 8965 & 6250 & 5912 & 6685 & 1.618 & 0.825 & 0.727 & 0.952 \\
\hline
\end{tabular}


Table G.3 Creep Test Results for 100\% Recycled and Blend ABS without Fibers 50\%

Sustained Load

\begin{tabular}{|c|c|c|c|c|c|}
\hline Date & \multirow{2}{*}{ Days } & \multicolumn{4}{|c|}{ Specimens } \\
\cline { 3 - 6 } & & Strain Reading $(\mu \mathbf{s})$ & Creep Coefficient \\
\cline { 3 - 6 } & & $\mathrm{A} 3$ & $\mathrm{~A} 5$ & $\mathrm{~A} 3$ & $\mathrm{~A} 5$ \\
\hline $02 / 28 / 2000$ & 0 & 9265 & 8770 & 0.000 & 0.000 \\
\hline $02 / 29 / 2000$ & 1 & 10801 & 10651 & 0.166 & 0.214 \\
\hline $03 / 01 / 2000$ & 2 & 11573 & 11402 & 0.249 & 0.300 \\
\hline $03 / 02 / 2000$ & 3 & 12146 & 12102 & 0.311 & 0.380 \\
\hline $03 / 03 / 2000$ & 4 & 12401 & 12396 & 0.338 & 0.413 \\
\hline $03 / 04 / 2000$ & 5 & 12711 & 12748 & 0.372 & 0.454 \\
\hline $03 / 05 / 2000$ & 6 & 13067 & 13172 & 0.410 & 0.502 \\
\hline $03 / 06 / 2000$ & 7 & 13193 & 13310 & 0.424 & 0.518 \\
\hline $03 / 07 / 2000$ & 8 & 13525 & 13670 & 0.460 & 0.559 \\
\hline $03 / 08 / 2000$ & 9 & 13926 & 14140 & 0.503 & 0.612 \\
\hline $03 / 09 / 2000$ & 10 & 14298 & 14651 & 0.543 & 0.671 \\
\hline $03 / 10 / 2000$ & 11 & 14463 & 14955 & 0.561 & 0.705 \\
\hline $03 / 11 / 2000$ & 12 & 14568 & 15088 & 0.572 & 0.720 \\
\hline $03 / 12 / 2000$ & 13 & 14706 & 15240 & 0.587 & 0.738 \\
\hline $03 / 13 / 2000$ & 14 & 14781 & 15352 & 0.595 & 0.751 \\
\hline $03 / 14 / 2000$ & 15 & 14849 & 15413 & 0.603 & 0.757 \\
\hline $03 / 15 / 2000$ & 16 & 15080 & 15692 & 0.628 & 0.789 \\
\hline $03 / 16 / 2000$ & 17 & 15319 & 15922 & 0.653 & 0.816 \\
\hline $03 / 17 / 2000$ & 18 & 15370 & 16025 & 0.659 & 0.827 \\
\hline $03 / 18 / 2000$ & 19 & 15397 & 16061 & 0.662 & 0.831 \\
\hline $03 / 19 / 2000$ & 20 & 15506 & 16192 & 0.674 & 0.846 \\
\hline $03 / 20 / 2000$ & 21 & 15639 & 16350 & 0.688 & 0.864 \\
\hline $03 / 21 / 2000$ & 22 & 15879 & 16565 & 0.714 & 0.889 \\
\hline $03 / 22 / 2000$ & 23 & 16085 & 16810 & 0.736 & 0.917 \\
\hline $03 / 23 / 2000$ & 24 & 16310 & 17069 & 0.760 & 0.946 \\
\hline $03 / 24 / 2000$ & 25 & 16558 & 17345 & 0.787 & 0.978 \\
\hline $03 / 25 / 2000$ & 26 & 16666 & 17525 & 0.799 & 0.998 \\
\hline $03 / 26 / 2000$ & 27 & 16675 & 17541 & 0.800 & 1.000 \\
\hline $03 / 27 / 2000$ & 28 & 16800 & 17672 & 0.813 & 1.015 \\
\hline $03 / 29 / 2000$ & 30 & 16891 & 17773 & 0.823 & 1.027 \\
\hline $03 / 30 / 2000$ & 31 & 16922 & 17821 & 0.826 & 1.032 \\
\hline $03 / 31 / 2000$ & 32 & 16921 & 17833 & 0.826 & 1.033 \\
\hline $04 / 03 / 2000$ & 35 & 17540 & 18536 & 0.893 & 1.114 \\
\hline $04 / 05 / 2000$ & 37 & 17672 & 18764 & 0.907 & 1.140 \\
\hline $04 / 07 / 2000$ & 39 & 17957 & 19087 & 0.938 & 1.176 \\
\hline & & Failure & Failure & Failure & Failure \\
\hline
\end{tabular}


Table G.4 Creep Test Results for Virgin ABS without Fibers; Virgin, Blend, and 100\%

Recycled with Fibers at 50\% Sustained Load

\begin{tabular}{|c|c|c|c|c|c|c|c|c|c|}
\hline \multirow[t]{3}{*}{ Date } & \multirow[t]{3}{*}{ Days } & \multicolumn{8}{|c|}{ Specimens } \\
\hline & & \multicolumn{4}{|c|}{ Strain Reading $(\mu \mathbf{s})$} & \multicolumn{4}{|c|}{ Creep Coefficient } \\
\hline & & A1 & A2 & A4 & A6 & A1 & A2 & A4 & A6 \\
\hline $01 / 11 / 2000$ & 0 & 9490 & & 3759 & 4102 & 0.000 & & 0.000 & 0.000 \\
\hline $01 / 12 / 2000$ & 1 & 11123 & & 4150 & 4471 & 0.172 & & 0.104 & 0.090 \\
\hline $01 / 13 / 2000$ & 2 & 11972 & 6514 & 4293 & 4278 & 0.262 & 0.000 & 0.142 & 0.043 \\
\hline $01 / 14 / 2000$ & 3 & 12483 & 6691 & 4363 & 4341 & 0.315 & 0.027 & 0.161 & 0.058 \\
\hline $01 / 15 / 2000$ & 4 & 12778 & 6694 & 4397 & 4372 & 0.346 & 0.028 & 0.170 & 0.066 \\
\hline $01 / 16 / 2000$ & 5 & 13171 & 6768 & 4462 & 4428 & 0.388 & 0.039 & 0.187 & 0.079 \\
\hline $01 / 17 / 2000$ & 6 & 13323 & 6790 & 4477 & 4433 & 0.404 & 0.042 & 0.191 & 0.081 \\
\hline $01 / 18 / 2000$ & 7 & 13577 & 6838 & 4518 & 4477 & 0.431 & 0.050 & 0.202 & 0.091 \\
\hline $01 / 19 / 2000$ & 8 & 13899 & 6896 & 4573 & 4528 & 0.465 & 0.059 & 0.217 & 0.104 \\
\hline $01 / 20 / 2000$ & 9 & 14159 & 6946 & 4617 & 4568 & 0.492 & 0.066 & 0.228 & 0.114 \\
\hline $01 / 21 / 2000$ & 10 & 14308 & 6967 & 4636 & 4581 & 0.508 & 0.070 & 0.233 & 0.117 \\
\hline $01 / 22 / 2000$ & 11 & 14519 & 6995 & 4666 & 4612 & 0.530 & 0.074 & 0.241 & 0.124 \\
\hline $01 / 23 / 2000$ & 12 & 14712 & 7031 & 4694 & 4636 & 0.550 & 0.079 & 0.249 & 0.130 \\
\hline $01 / 24 / 2000$ & 13 & 14909 & 7067 & 4730 & 4673 & 0.571 & 0.085 & 0.258 & 0.139 \\
\hline $01 / 25 / 2000$ & 14 & 15105 & 7098 & 4758 & 4698 & 0.592 & 0.090 & 0.266 & 0.145 \\
\hline $01 / 26 / 2000$ & 15 & 15131 & 7096 & 4755 & 4694 & 0.594 & 0.089 & 0.265 & 0.144 \\
\hline $01 / 27 / 2000$ & 16 & 15291 & 7122 & 4777 & 4715 & 0.611 & 0.093 & 0.271 & 0.149 \\
\hline $01 / 28 / 2000$ & 17 & 15367 & 7124 & 4779 & 4712 & 0.619 & 0.094 & 0.271 & 0.149 \\
\hline $01 / 29 / 2000$ & 18 & 15505 & 7150 & 4800 & 4732 & 0.634 & 0.098 & 0.277 & 0.154 \\
\hline $01 / 30 / 2000$ & 19 & 15749 & 7197 & 4845 & 4775 & 0.660 & 0.105 & 0.289 & 0.164 \\
\hline $01 / 31 / 2000$ & 20 & 15889 & 7217 & 4868 & 4795 & 0.674 & 0.108 & 0.295 & 0.169 \\
\hline $02 / 01 / 2000$ & 21 & 16021 & 7239 & 4891 & 4820 & 0.688 & 0.111 & 0.301 & 0.175 \\
\hline $02 / 02 / 2000$ & 22 & 16106 & 7252 & 4899 & 4826 & 0.697 & 0.113 & 0.303 & 0.176 \\
\hline $02 / 03 / 2000$ & 23 & 16132 & 7240 & 4898 & 4818 & 0.700 & 0.111 & 0.303 & 0.175 \\
\hline $02 / 04 / 2000$ & 24 & 16416 & 7307 & 4956 & 4876 & 0.730 & 0.122 & 0.318 & 0.189 \\
\hline $02 / 05 / 2000$ & 25 & 16459 & 7308 & 4959 & 4878 & 0.734 & 0.122 & 0.319 & 0.189 \\
\hline $02 / 06 / 2000$ & 26 & 16538 & 7317 & 4970 & 4888 & 0.743 & 0.123 & 0.322 & 0.192 \\
\hline $02 / 07 / 2000$ & 27 & 16652 & 7337 & 4991 & 4907 & 0.755 & 0.126 & 0.328 & 0.196 \\
\hline $02 / 08 / 2000$ & 28 & 16748 & 7352 & 5000 & 4920 & 0.765 & 0.129 & 0.330 & 0.199 \\
\hline $02 / 09 / 2000$ & 29 & 16873 & 7374 & 5022 & 4937 & 0.778 & 0.132 & 0.336 & 0.204 \\
\hline $02 / 10 / 2000$ & 30 & 17000 & 7392 & 5045 & 4957 & 0.791 & 0.135 & 0.342 & 0.208 \\
\hline $02 / 11 / 2000$ & 31 & 17200 & 7431 & 5081 & 4992 & 0.812 & 0.141 & 0.352 & 0.217 \\
\hline $02 / 12 / 2000$ & 32 & 17264 & 7430 & 5083 & 4995 & 0.819 & 0.141 & 0.352 & 0.218 \\
\hline $02 / 13 / 2000$ & 33 & 17350 & 7444 & 5097 & 5006 & 0.828 & 0.143 & 0.356 & 0.220 \\
\hline $02 / 14 / 2000$ & 34 & 17624 & 7493 & 5151 & 5053 & 0.857 & 0.150 & 0.370 & 0.232 \\
\hline $02 / 15 / 2000$ & 35 & 17661 & 7495 & 5151 & 5055 & 0.861 & 0.151 & 0.370 & 0.232 \\
\hline 02/16/2000 & 36 & 17845 & 7526 & 5182 & 5081 & 0.880 & 0.155 & 0.379 & 0.239 \\
\hline $02 / 17 / 2000$ & 37 & 17844 & 7521 & 5179 & 5078 & 0.880 & 0.155 & 0.378 & 0.238 \\
\hline $02 / 18 / 2000$ & 38 & 17945 & 7533 & 5188 & 5088 & 0.891 & 0.156 & 0.380 & 0.240 \\
\hline $02 / 19 / 2000$ & 39 & 18158 & 7567 & 5227 & 5123 & 0.913 & 0.162 & 0.391 & 0.249 \\
\hline $02 / 20 / 2000$ & 40 & 18188 & 7561 & 5226 & 5117 & 0.917 & 0.161 & 0.390 & 0.247 \\
\hline $02 / 21 / 2000$ & 41 & 18324 & 7587 & 5247 & 5143 & 0.931 & 0.165 & 0.396 & 0.254 \\
\hline
\end{tabular}




\begin{tabular}{|c|c|c|c|c|c|c|c|c|c|}
\hline $02 / 22 / 2000$ & 42 & 18490 & 7622 & 5283 & 5176 & 0.948 & 0.170 & 0.405 & 0.262 \\
\hline $02 / 23 / 2000$ & 43 & 18720 & 7666 & 5328 & 5217 & 0.973 & 0.177 & 0.417 & 0.272 \\
\hline $02 / 24 / 2000$ & 44 & 18938 & 7698 & 5363 & 5253 & 0.996 & 0.182 & 0.427 & 0.281 \\
\hline $02 / 25 / 2000$ & 45 & Failure & 7778 & 5433 & 5322 & Failure & 0.194 & 0.445 & 0.297 \\
\hline $02 / 26 / 2000$ & 46 & & 7821 & 5498 & 5375 & & 0.201 & 0.463 & 0.310 \\
\hline $02 / 27 / 2000$ & 47 & & 7826 & 5500 & 5376 & & 0.201 & 0.463 & 0.311 \\
\hline $02 / 28 / 2000$ & 48 & & 7820 & 5492 & 5368 & & 0.200 & 0.461 & 0.309 \\
\hline $02 / 29 / 2000$ & 49 & & 7818 & 5493 & 5364 & & 0.200 & 0.461 & 0.308 \\
\hline $03 / 01 / 2000$ & 50 & & 7838 & 5513 & 5384 & & 0.203 & 0.467 & 0.313 \\
\hline $03 / 02 / 2000$ & 51 & & 7836 & 5515 & 5384 & & 0.203 & 0.467 & 0.313 \\
\hline $03 / 03 / 2000$ & 52 & & 7844 & 5517 & 5389 & & 0.204 & 0.468 & 0.314 \\
\hline $03 / 04 / 2000$ & 53 & & 7846 & 5524 & 5393 & & 0.204 & 0.470 & 0.315 \\
\hline $03 / 05 / 2000$ & 54 & & 7858 & 5534 & 5401 & & 0.206 & 0.472 & 0.317 \\
\hline $03 / 06 / 2000$ & 55 & & 7879 & 5559 & 5422 & & 0.210 & 0.479 & 0.322 \\
\hline $03 / 07 / 2000$ & 56 & & 7909 & 5590 & 5456 & & 0.214 & 0.487 & 0.330 \\
\hline $03 / 08 / 2000$ & 57 & & 7971 & 5650 & 5513 & & 0.224 & 0.503 & 0.344 \\
\hline $03 / 09 / 2000$ & 58 & & 8000 & 5687 & 5546 & & 0.228 & 0.513 & 0.352 \\
\hline $03 / 10 / 2000$ & 59 & & 7976 & 5656 & 5520 & & 0.224 & 0.505 & 0.346 \\
\hline $03 / 11 / 2000$ & 60 & & 7986 & 5678 & 5533 & & 0.226 & 0.511 & 0.349 \\
\hline $03 / 12 / 2000$ & 61 & & 7968 & 5657 & 5515 & & 0.223 & 0.505 & 0.344 \\
\hline $03 / 13 / 2000$ & 62 & & 7974 & 5664 & 5521 & & 0.224 & 0.507 & 0.346 \\
\hline $03 / 14 / 2000$ & 63 & & 7974 & 5668 & 5520 & & 0.224 & 0.508 & 0.346 \\
\hline $03 / 15 / 2000$ & 64 & & 8004 & 5695 & 5546 & & 0.229 & 0.515 & 0.352 \\
\hline $03 / 16 / 2000$ & 65 & & 8029 & 5720 & 5569 & & 0.233 & 0.522 & 0.358 \\
\hline $03 / 17 / 2000$ & 66 & & 8010 & 5701 & 5550 & & 0.230 & 0.517 & 0.353 \\
\hline $03 / 18 / 2000$ & 67 & & 8005 & 5696 & 5545 & & 0.229 & 0.515 & 0.352 \\
\hline $03 / 19 / 2000$ & 68 & & 8015 & 5707 & 5546 & & 0.230 & 0.518 & 0.352 \\
\hline $03 / 20 / 2000$ & 69 & & 8032 & 5724 & 5573 & & 0.233 & 0.523 & 0.359 \\
\hline $03 / 21 / 2000$ & 70 & & 8054 & 5751 & 5599 & & 0.236 & 0.530 & 0.365 \\
\hline $03 / 22 / 2000$ & 71 & & 8086 & 5783 & 5629 & & 0.241 & 0.538 & 0.372 \\
\hline $03 / 23 / 2000$ & 72 & & 8122 & 5820 & 5663 & & 0.247 & 0.548 & 0.381 \\
\hline $03 / 24 / 2000$ & 73 & & 8157 & 5854 & 5696 & & 0.252 & 0.557 & 0.389 \\
\hline $03 / 25 / 2000$ & 74 & & 8155 & 5857 & 5697 & & 0.252 & 0.558 & 0.389 \\
\hline $03 / 26 / 2000$ & 75 & & 8143 & 5845 & 5683 & & 0.250 & 0.555 & 0.385 \\
\hline $03 / 27 / 2000$ & 76 & & 8150 & 5856 & 5694 & & 0.251 & 0.558 & 0.388 \\
\hline $03 / 29 / 2000$ & 78 & & 8147 & 5856 & 5688 & & 0.251 & 0.558 & 0.387 \\
\hline $03 / 30 / 2000$ & 79 & & 8144 & 5854 & 5686 & & 0.250 & 0.557 & 0.386 \\
\hline $03 / 31 / 2000$ & 80 & & 8138 & 5849 & 5679 & & 0.249 & 0.556 & 0.384 \\
\hline $04 / 03 / 2000$ & 83 & & 8246 & 5960 & 5787 & & 0.266 & 0.586 & 0.411 \\
\hline $04 / 05 / 2000$ & 85 & & 8241 & 5958 & 5782 & & 0.265 & 0.585 & 0.410 \\
\hline $04 / 07 / 2000$ & 87 & & 8274 & 5994 & 5814 & & 0.270 & 0.595 & 0.417 \\
\hline $04 / 10 / 2000$ & 90 & & 8277 & 6000 & 5814 & & 0.271 & 0.596 & 0.417 \\
\hline $04 / 11 / 2000$ & 91 & & 8279 & 6003 & 5814 & & 0.271 & 0.597 & 0.417 \\
\hline $04 / 13 / 2000$ & 93 & & 8304 & 6038 & 5841 & & 0.275 & 0.606 & 0.424 \\
\hline $04 / 14 / 2000$ & 94 & & 8327 & 6053 & 5862 & & 0.278 & 0.610 & 0.429 \\
\hline $04 / 17 / 2000$ & 97 & & 8340 & 6082 & 5896 & & 0.280 & 0.618 & 0.437 \\
\hline $04 / 18 / 2000$ & 98 & & 8396 & 6136 & 5936 & & 0.289 & 0.632 & 0.447 \\
\hline $04 / 20 / 2000$ & 100 & & 8480 & 6219 & 6015 & & 0.302 & 0.654 & 0.466 \\
\hline
\end{tabular}




\begin{tabular}{|c|c|c|c|c|c|c|c|}
\hline $04 / 21 / 2000$ & 101 & 8502 & 6242 & 6038 & 0.305 & 0.661 & 0.472 \\
\hline $04 / 24 / 2000$ & 104 & 8460 & 6207 & 6004 & 0.299 & 0.651 & 0.464 \\
\hline $04 / 26 / 2000$ & 106 & 8472 & 6218 & 6007 & 0.301 & 0.654 & 0.464 \\
\hline $04 / 28 / 2000$ & 108 & 8489 & 6234 & 6024 & 0.303 & 0.658 & 0.469 \\
\hline $05 / 01 / 2000$ & 111 & 8542 & 6278 & 6064 & 0.311 & 0.670 & 0.478 \\
\hline $05 / 03 / 2000$ & 113 & 8564 & 6312 & 6102 & 0.315 & 0.679 & 0.488 \\
\hline $05 / 05 / 2000$ & 115 & 8671 & 6426 & 6209 & 0.331 & 0.709 & 0.514 \\
\hline $05 / 08 / 2000$ & 118 & 8780 & 6558 & 6324 & 0.348 & 0.745 & 0.542 \\
\hline $05 / 10 / 2000$ & 120 & 8790 & 6582 & 6335 & 0.349 & 0.751 & 0.544 \\
\hline $05 / 12 / 2000$ & 122 & 8843 & 6644 & 6390 & 0.358 & 0.767 & 0.558 \\
\hline $05 / 15 / 2000$ & 125 & 8817 & 6637 & 6363 & 0.354 & 0.766 & 0.551 \\
\hline $05 / 17 / 2000$ & 127 & 8857 & 6674 & 6405 & 0.360 & 0.775 & 0.561 \\
\hline $05 / 19 / 2000$ & 129 & 8890 & 6715 & 6443 & 0.365 & 0.786 & 0.571 \\
\hline $05 / 22 / 2000$ & 132 & 8925 & 6765 & 6480 & 0.370 & 0.800 & 0.580 \\
\hline $05 / 26 / 2000$ & 136 & 8969 & 6809 & 6525 & 0.377 & 0.811 & 0.591 \\
\hline $05 / 30 / 2000$ & 140 & 8962 & 6814 & 6526 & 0.376 & 0.813 & 0.591 \\
\hline $06 / 01 / 2000$ & 142 & 9106 & 6959 & 6661 & 0.398 & 0.851 & 0.624 \\
\hline $06 / 02 / 2000$ & 143 & 9153 & 7009 & 6713 & 0.405 & 0.865 & 0.637 \\
\hline $06 / 05 / 2000$ & 146 & 9124 & 6997 & 6685 & 0.401 & 0.861 & 0.630 \\
\hline $06 / 07 / 2000$ & 148 & 9096 & 6970 & 6658 & 0.396 & 0.854 & 0.623 \\
\hline $06 / 09 / 2000$ & 150 & 9185 & 7065 & 6688 & 0.410 & 0.879 & 0.630 \\
\hline $06 / 12 / 2000$ & 153 & 9286 & 7180 & 6315 & 0.426 & 0.910 & 0.539 \\
\hline $06 / 14 / 2000$ & 155 & 9388 & 7300 & 6418 & 0.441 & 0.942 & 0.565 \\
\hline $06 / 16 / 2000$ & 157 & 9491 & 7461 & 6533 & 0.457 & 0.985 & 0.593 \\
\hline $06 / 21 / 2000$ & 162 & 9503 & 7483 & 6543 & 0.459 & 0.991 & 0.595 \\
\hline $06 / 23 / 2000$ & 164 & 9582 & 7570 & 6618 & 0.471 & 1.014 & 0.613 \\
\hline $06 / 26 / 2000$ & 167 & 9601 & 7608 & 6641 & 0.474 & 1.024 & 0.619 \\
\hline $06 / 28 / 2000$ & 169 & 9689 & 7655 & 6689 & 0.487 & 1.036 & 0.631 \\
\hline $07 / 06 / 2000$ & 177 & 9746 & 7733 & 6753 & 0.496 & 1.057 & 0.646 \\
\hline $07 / 12 / 2000$ & 183 & 9824 & 7810 & 6876 & 0.508 & 1.078 & 0.676 \\
\hline $07 / 17 / 2000$ & 188 & 9794 & 7813 & 6821 & 0.504 & 1.078 & 0.663 \\
\hline $07 / 22 / 2000$ & 193 & 9947 & 7993 & 6973 & 0.527 & 1.126 & 0.700 \\
\hline $07 / 27 / 2000$ & 198 & 10062 & 8093 & 7067 & 0.545 & 1.153 & 0.723 \\
\hline $07 / 31 / 2000$ & 202 & 9951 & - & - & 0.528 & - & - \\
\hline $08 / 04 / 2000$ & 206 & 10048 & - & - & 0.543 & - & - \\
\hline $08 / 09 / 2000$ & 211 & 10041 & - & - & 0.541 & - & - \\
\hline $08 / 14 / 2000$ & 216 & 9971 & - & - & 0.531 & - & - \\
\hline $08 / 19 / 2000$ & 221 & 10072 & - & - & 0.546 & - & - \\
\hline $08 / 28 / 2000$ & 230 & 10105 & - & - & 0.551 & - & - \\
\hline $09 / 06 / 2000$ & 239 & 10133 & - & - & 0.556 & - & - \\
\hline $09 / 11 / 2000$ & 244 & 10352 & - & - & 0.589 & - & - \\
\hline $09 / 15 / 2000$ & 248 & 10302 & - & - & 0.582 & - & - \\
\hline $09 / 18 / 2000$ & 251 & 10302 & - & - & 0.582 & - & - \\
\hline $09 / 22 / 2000$ & 255 & 10309 & - & - & 0.583 & - & - \\
\hline $09 / 25 / 2000$ & 258 & 10301 & - & - & 0.581 & - & - \\
\hline $10 / 02 / 2000$ & 265 & 10307 & - & - & 0.582 & - & - \\
\hline $10 / 05 / 2000$ & 268 & 10512 & - & - & 0.614 & - & - \\
\hline $10 / 08 / 2000$ & 271 & 10696 & - & - & 0.642 & - & - \\
\hline
\end{tabular}




\begin{tabular}{|c|c|c|c|c|c|c|c|c|c|}
\hline $10 / 13 / 2000$ & 276 & & 10815 & - & - & & 0.660 & - & - \\
\hline $10 / 18 / 2000$ & 281 & & 11026 & - & - & & 0.693 & - & - \\
\hline $02 / 08 / 2001$ & 394 & & 11758 & 8542 & 7965 & & 0.805 & 1.272 & 0.942 \\
\hline $05 / 15 / 2001$ & 490 & & 12384 & 8853 & 8254 & & 0.901 & 1.355 & 1.012 \\
\hline $07 / 12 / 2001$ & 548 & & 13261 & 9114 & 8482 & & 1.036 & 1.425 & 1.068 \\
\hline $10 / 17 / 2001$ & 645 & & 13967 & 9658 & 8897 & & 1.144 & 1.569 & 1.169 \\
\hline $01 / 16 / 2002$ & 736 & & 14563 & 9928 & 9623 & & 1.236 & 1.641 & 1.346 \\
\hline $04 / 12 / 2002$ & 822 & & 15047 & 10857 & 10021 & & 1.310 & 1.888 & 1.443 \\
\hline $06 / 04 / 2002$ & 875 & & 16352 & 11406 & 10524 & & 1.510 & 2.034 & 1.566 \\
\hline $08 / 12 / 2002$ & 944 & & 16879 & 11953 & 11065 & & 1.591 & 2.180 & 1.697 \\
\hline
\end{tabular}


Table G.5 Creep Test Results for Virgin, Blend, and 100\% Recycled PC with and without

Fibers at 20\% Sustained Load

\begin{tabular}{|c|c|c|c|c|c|c|c|c|c|c|c|c|c|}
\hline \multirow[t]{3}{*}{ Date } & \multirow[t]{3}{*}{ Days } & \multicolumn{12}{|c|}{ Specimens } \\
\hline & & \multicolumn{6}{|c|}{ Strain Reading ( $\mu \mathrm{s})$} & \multicolumn{6}{|c|}{ Creep Coefficient } \\
\hline & & P1 & $\mathbf{P 2}$ & P3 & P4 & P5 & P6 & $\mathbf{P 1}$ & $\mathbf{P 2}$ & P3 & $\overline{\text { P4 }}$ & P5 & P6 \\
\hline $05 / 25 / 2000$ & 0 & & & & & & & & & & & & \\
\hline $05 / 26 / 2000$ & 1 & 15060 & 8816 & 5232 & 7250 & 15660 & 8335 & 0.000 & 0.000 & 0.000 & 0.000 & 0.000 & 0.000 \\
\hline $05 / 27 / 2000$ & 2 & 16275 & 9125 & 17185 & 7565 & 16690 & 8651 & 0.081 & 0.035 & 0.128 & 0.043 & 0.066 & 0.038 \\
\hline $05 / 28 / 2000$ & 3 & 16859 & 9282 & 17699 & 7733 & 17191 & 8815 & 0.119 & 0.053 & 0.162 & 0.067 & 0.098 & 0.058 \\
\hline 05/29/2000 & 4 & 17245 & 9391 & 18032 & 7855 & 17536 & 8932 & 0.145 & 0.065 & 0.184 & \begin{tabular}{|l|}
0.083 \\
\end{tabular} & 0.120 & 0.072 \\
\hline $05 / 30 / 2000$ & 5 & 17436 & 9444 & 18177 & 7928 & 17711 & 8995 & 0.158 & 0.071 & 0.193 & \begin{tabular}{|l|}
0.094 \\
\end{tabular} & 0.131 & 0.079 \\
\hline $05 / 31 / 2000$ & 6 & 17679 & 9520 & 18399 & 8006 & 17958 & 9077 & 0.174 & 0.080 & 0.208 & 0.104 & 0.147 & 0.089 \\
\hline $06 / 01 / 2000$ & 7 & 18028 & 9609 & 18707 & 8108 & 18306 & 9173 & 0.197 & 0.090 & 0.228 & 0.118 & 0.169 & 0.101 \\
\hline $06 / 02 / 2000$ & 8 & 18410 & 9718 & 19038 & 8249 & 18886 & 9312 & 0.222 & 0.102 & 0.250 & 0.138 & 0.206 & 0.117 \\
\hline $06 / 03 / 2000$ & 9 & 18601 & 9772 & 19184 & 8332 & 19134 & 9409 & 0.235 & 0.108 & 0.259 & 0.149 & 0.222 & 0.129 \\
\hline $06 / 04 / 2000$ & 10 & 18681 & 9820 & 19249 & 8380 & 19424 & 9476 & 0.240 & 0.114 & 0.264 & \begin{tabular}{|l|}
0.156 \\
\end{tabular} & 0.240 & 0.137 \\
\hline $06 / 05 / 2000$ & 11 & 18798 & 9853 & 19336 & 8414 & 19551 & 9518 & 0.248 & 0.118 & 0.269 & 0.161 & 0.248 & 0.142 \\
\hline $06 / 06 / 2000$ & 12 & 18930 & 9895 & 19450 & 8455 & 19689 & 9566 & 0.257 & 0.122 & 0.277 & 0.166 & 0.257 & 0.148 \\
\hline $06 / 07 / 2000$ & 13 & 18845 & 9885 & 19404 & 8455 & 19650 & 9564 & 0.251 & 0.121 & 0.274 & 0.166 & 0.255 & 0.147 \\
\hline $06 / 08 / 2000$ & 14 & 18960 & 9909 & 19460 & 8480 & 19740 & 9594 & 0.259 & 0.124 & 0.278 & 0.170 & 0.261 & 0.151 \\
\hline 06/09/2000 & 15 & 18998 & 9928 & 19486 & 8498 & 19810 & 9605 & 0.261 & 0.126 & 0.279 & 0.172 & 0.265 & 0.152 \\
\hline $06 / 10 / 2000$ & 16 & 19306 & 10004 & 19790 & 8585 & 20113 & 9690 & 0.282 & 0.135 & 0.299 & 0.184 & 0.284 & 0.163 \\
\hline $06 / 10 / 2000$ & 16 & 19345 & 10015 & 19832 & 8601 & 20166 & 9703 & 0.285 & 0.136 & 0.302 & 0.186 & 0.288 & 0.164 \\
\hline $06 / 12 / 2000$ & 18 & 19622 & 10093 & 20053 & 8683 & 20451 & 9789 & 0.303 & 0.145 & 0.317 & 0.198 & 0.306 & 0.174 \\
\hline $06 / 13 / 2000$ & 19 & 19646 & 10115 & 20048 & 8702 & 20579 & 9817 & 0.305 & 0.147 & 0.316 & 0.200 & 0.314 & 0.178 \\
\hline $06 / 14 / 2000$ & 20 & 19828 & 10168 & 20213 & 8764 & 20868 & 9891 & 0.317 & 0.153 & 0.327 & 0.209 & 0.333 & 0.187 \\
\hline $06 / 15 / 2000$ & 21 & 19911 & 10207 & 20290 & 8802 & 20753 & 9958 & 0.322 & 0.158 & 0.332 & 0.214 & 0.325 & 0.195 \\
\hline $06 / 16 / 2000$ & 22 & 19968 & 10236 & 20329 & 8833 & 20807 & 9990 & 0.326 & 0.161 & 0.335 & 0.218 & 0.329 & 0.199 \\
\hline $06 / 17 / 2000$ & 23 & 20159 & 10281 & 20518 & 8862 & 21000 & 10015 & 0.339 & 0.166 & 0.347 & 0.222 & 0.341 & 0.202 \\
\hline $06 / 18 / 2000$ & 24 & 20206 & 10355 & 21876 & Failure & 21232 & 10152 & 0.342 & 0.175 & 0.436 & Failure & 0.356 & 0.218 \\
\hline $06 / 20 / 2000$ & 26 & 20467 & 10370 & 20727 & Failure & 21279 & 10337 & 0.359 & 0.176 & 0.361 & & 0.359 & 0.240 \\
\hline $06 / 21 / 2000$ & 27 & 20512 & 10428 & 20824 & Failure & 21380 & 10218 & 0.362 & 0.183 & 0.367 & & 0.365 & 0.226 \\
\hline $06 / 22 / 2000$ & 28 & 20426 & 10418 & 20730 & Failure & 21276 & 10210 & 0.356 & 0.182 & 0.361 & & 0.359 & 0.225 \\
\hline $06 / 23 / 2000$ & 29 & 20388 & 10402 & 20668 & Failure & 21245 & \begin{tabular}{|l|}
10193 \\
\end{tabular} & 0.354 & 0.180 & 0.357 & & 0.357 & 0.223 \\
\hline $06 / 24 / 2000$ & 30 & 20412 & 10398 & 19002 & Failure & 21248 & 10216 & 0.355 & 0.179 & 0.248 & & 0.357 & 0.226 \\
\hline $06 / 25 / 2000$ & 31 & 20436 & 10403 & 20916 & Failure & 21361 & 10270 & 0.357 & 0.180 & 0.373 & & 0.364 & 0.232 \\
\hline $06 / 26 / 2000$ & 32 & 20976 & 10531 & 21000 & \begin{tabular}{|l} 
Failure \\
\end{tabular} & 22151 & 10301 & 0.393 & 0.195 & 0.379 & & 0.414 & 0.236 \\
\hline $06 / 26 / 2000$ & 32 & 20849 & 10545 & 21111 & Failure & 21724 & 10337 & 0.384 & 0.196 & 0.386 & & 0.387 & 0.240 \\
\hline $06 / 27 / 2000$ & 33 & 20641 & 10511 & 20891 & Failure & 21446 & 10110 & 0.371 & 0.192 & 0.372 & & 0.369 & 0.213 \\
\hline $06 / 28 / 2000$ & 34 & 20701 & 10538 & 20097 & Failure & 21602 & 10321 & 0.375 & 0.195 & 0.319 & & 0.379 & 0.238 \\
\hline $06 / 29 / 2000$ & 35 & 20676 & 10483 & 20918 & Failure & 21546 & 10310 & 0.373 & 0.189 & 0.373 & & 0.376 & 0.237 \\
\hline $07 / 06 / 2000$ & 42 & 21081 & 10619 & 21206 & Failure & 21893 & 10436 & 0.400 & 0.205 & 0.392 & & 0.398 & 0.252 \\
\hline $07 / 07 / 2000$ & 43 & 20653 & 10610 & 21153 & Failure & 21786 & \begin{tabular}{|l|}
10314 \\
\end{tabular} & 0.371 & 0.203 & 0.389 & & 0.391 & 0.237 \\
\hline $07 / 09 / 2000$ & 45 & 21177 & 10679 & 21465 & Failure & 22101 & 10450 & 0.406 & 0.211 & 0.409 & & 0.411 & 0.254 \\
\hline $07 / 12 / 2000$ & 48 & 21049 & 10648 & 21330 & Failure & 21970 & 10427 & 0.398 & 0.208 & 0.400 & & 0.403 & 0.251 \\
\hline $07 / 13 / 2000$ & 49 & 21161 & 10677 & 21441 & Failure & 22066 & 10454 & 0.405 & 0.211 & 0.408 & & 0.409 & 0.254 \\
\hline $07 / 14 / 2000$ & 50 & 21112 & 10677 & 21346 & Failure & 22018 & 10456 & 0.402 & 0.211 & 0.401 & & 0.406 & 0.254 \\
\hline
\end{tabular}




\begin{tabular}{|c|c|c|c|c|c|c|c|c|c|c|c|c|}
\hline $07 / 18 / 2000$ & 54 & 21324 & 10746 & 21641 & Failure & 22293 & 10522 & 0.416 & 0.219 & 0.421 & 0.424 & 0.262 \\
\hline $07 / 22 / 2000$ & 58 & 21284 & 10745 & 21519 & Failure & 22178 & 10503 & 0.413 & 0.219 & 0.413 & 0.416 & 0.260 \\
\hline $07 / 24 / 2000$ & 60 & 21330 & 10741 & 21580 & Failure & 22247 & 10518 & 0.416 & 0.218 & 0.417 & 0.421 & 0.262 \\
\hline $07 / 26 / 2000$ & 62 & 21306 & 10720 & 21461 & Failure & 22101 & 10508 & 0.415 & 0.216 & 0.409 & 0.411 & 0.261 \\
\hline $07 / 28 / 2000$ & 64 & 21443 & 10748 & 21534 & Failure & \begin{tabular}{|l|}
22194 \\
\end{tabular} & 10524 & 0.424 & 0.219 & 0.414 & 0.417 & 0.263 \\
\hline $07 / 30 / 2000$ & 66 & 21602 & 10825 & 21786 & Failure & 22475 & 10602 & 0.434 & 0.228 & 0.430 & 0.435 & 0.272 \\
\hline $08 / 04 / 2000$ & 71 & 21530 & 10829 & 21751 & Failure & 22454 & 10601 & 0.430 & 0.228 & 0.428 & 0.434 & 0.272 \\
\hline $08 / 06 / 2000$ & 73 & 21485 & 10838 & 21697 & Failure & 22406 & 10608 & 0.427 & 0.229 & 0.424 & 0.431 & 0.273 \\
\hline $08 / 08 / 2000$ & 75 & 21741 & 10934 & 21633 & Failure & 22626 & 10684 & 0.444 & 0.240 & 0.420 & 0.445 & 0.282 \\
\hline $08 / 11 / 2000$ & 78 & 21649 & 10930 & 21852 & Failure & 22589 & 10675 & 0.438 & 0.240 & 0.435 & 0.442 & 0.281 \\
\hline $08 / 15 / 2000$ & 82 & 21731 & 10947 & 21920 & Failure & 22668 & 10691 & 0.443 & 0.242 & 0.439 & 0.448 & 0.283 \\
\hline $08 / 19 / 2000$ & 86 & 21536 & 10935 & 21742 & Failure & 22460 & 10647 & 0.430 & 0.240 & 0.427 & 0.434 & 0.277 \\
\hline $08 / 23 / 2000$ & 90 & 21671 & 10952 & 21862 & Failure & 22524 & 10686 & 0.439 & 0.242 & 0.435 & 0.438 & 0.282 \\
\hline $08 / 28 / 2000$ & 95 & 21713 & 10987 & 21868 & Failure & 22719 & 10669 & 0.442 & 0.246 & 0.436 & 0.451 & 0.280 \\
\hline $09 / 06 / 2000$ & 104 & 21705 & 10991 & 21879 & Failure & 22674 & 10718 & 0.441 & 0.247 & 0.436 & 0.448 & 0.286 \\
\hline $09 / 11 / 2000$ & 109 & 22011 & 11072 & 21772 & Failure & 23100 & 10751 & 0.462 & 0.256 & 0.429 & 0.475 & 0.290 \\
\hline $09 / 15 / 2000$ & 113 & 21638 & 11099 & 21898 & Failure & 22688 & 10717 & 0.437 & 0.259 & 0.438 & 0.449 & 0.286 \\
\hline $09 / 18 / 2000$ & 116 & 21757 & 11021 & 21907 & Failure & 22793 & 10731 & 0.445 & 0.250 & 0.438 & 0.455 & 0.287 \\
\hline $09 / 22 / 2000$ & 120 & 21790 & \begin{tabular}{|l|}
11034 \\
\end{tabular} & 21800 & \begin{tabular}{|l|} 
Failure \\
\end{tabular} & 22808 & 10751 & 0.447 & 0.252 & 0.431 & 0.456 & 0.290 \\
\hline $09 / 25 / 2000$ & 123 & 22191 & 11023 & 21884 & Failure & 22631 & 10688 & 0.474 & 0.250 & 0.437 & 0.445 & 0.282 \\
\hline $10 / 02 / 2000$ & 130 & 21899 & 11032 & 21854 & Failure & 22732 & 10743 & 0.454 & 0.251 & 0.435 & 0.452 & 0.289 \\
\hline 10/05/2000 & 133 & 22053 & 11099 & 21856 & Failure & 23110 & 10817 & 0.464 & 0.259 & 0.435 & 0.476 & 0.298 \\
\hline $10 / 08 / 2000$ & 136 & 22102 & 11132 & 21859 & Failure & 23154 & 10858 & 0.468 & 0.263 & 0.435 & 0.479 & 0.303 \\
\hline $10 / 13 / 2000$ & 141 & 22142 & \begin{tabular}{|l|}
11178 \\
\end{tabular} & 21863 & \begin{tabular}{|l|} 
Failure \\
\end{tabular} & \begin{tabular}{|l|}
23189 \\
\end{tabular} & 10889 & 0.470 & 0.268 & 0.435 & 0.481 & 0.306 \\
\hline $10 / 18 / 2000$ & 146 & 22185 & 11201 & 21867 & Failure & 23231 & 10954 & 0.473 & 0.271 & 0.436 & 0.483 & 0.314 \\
\hline $02 / 08 / 2001$ & 259 & 22219 & 11356 & 21989 & & 23312 & 11056 & 0.475 & 0.288 & 0.444 & 0.489 & 0.326 \\
\hline $05 / 15 / 2001$ & 355 & 22257 & 11385 & 22369 & & 23369 & 11087 & 0.478 & 0.291 & 0.469 & 0.492 & 0.330 \\
\hline $07 / 12 / 2001$ & 413 & 22305 & 11474 & 22440 & & 23408 & 11145 & 0.481 & 0.301 & 0.473 & 0.495 & 0.337 \\
\hline 10/17/2001 & 510 & 22338 & 11499 & 22587 & & 23456 & 11235 & 0.483 & 0.304 & 0.483 & 0.498 & 0.348 \\
\hline $01 / 16 / 2002$ & 601 & 22387 & 11554 & 22627 & & 23486 & 11281 & 0.487 & 0.311 & 0.485 & 0.500 & 0.353 \\
\hline $04 / 12 / 2002$ & 687 & 22456 & 11663 & 22687 & & 23547 & 11356 & 0.491 & 0.323 & 0.489 & 0.504 & 0.362 \\
\hline $06 / 04 / 2002$ & 740 & 22515 & 11748 & 22776 & & 23689 & 11389 & 0.495 & 0.333 & 0.495 & 0.513 & 0.366 \\
\hline $08 / 12 / 2002$ & 809 & 22546 & 11854 & 22865 & & 23704 & 11423 & 0.497 & 0.345 & 0.501 & 0.514 & 0.370 \\
\hline
\end{tabular}


Table G.6 Creep Test Results for Virgin, Blend, and 100\% Recycled PC with and without

Fibers at $50 \%$ Sustained Load

\begin{tabular}{|c|c|c|c|c|c|c|c|c|c|c|c|c|c|}
\hline \multirow[t]{3}{*}{ Date } & \multirow[t]{3}{*}{ Jays } & \multicolumn{12}{|c|}{ Specimens } \\
\hline & & \multicolumn{6}{|c|}{ Strain Reading $(\mu s)$} & \multicolumn{6}{|c|}{ Creep Coefficient } \\
\hline & & $\overline{P 1}$ & $\mathrm{P} 2$ & P3 & P4 & P5 & $\bar{P} 6$ & $\mathrm{P} 1$ & $\mathrm{P} 2$ & P3 & $\overline{\mathrm{P} 4}$ & P5 & P6 \\
\hline 00 & 0 & 4151 & & 2980 & & 4434 & & 0.000 & & 0.000 & & 0.000 & \\
\hline $6 / 2000$ & $\overline{1}$ & $\overline{921}$ & 176 & $\overline{544}$ & $\overline{88}$ & 5232 & 3021 & 0.185 & 0.000 & 0.189 & 0.000 & 0.180 & .000 \\
\hline $5 / 27 / 2000$ & 2 & 170 & 230 & 3748 & 3141 & 5408 & 3076 & 0.245 & 0.017 & 0.258 & 0.017 & 0.220 & $\overline{.018}$ \\
\hline $5 / 28 / 2000$ & 3 & 312 & 3266 & 3857 & 3183 & 5495 & 3116 & 0.280 & 0.028 & 0.294 & 0.031 & 0.239 & 0.031 \\
\hline$\overline{000}$ & 4 & 421 & 3295 & 3949 & 213 & 5569 & 3146 & 0.306 & 0.037 & 0.325 & 0.040 & 0.256 & 0.041 \\
\hline $5 / 30 / 2000$ & 5 & 5452 & 3295 & 3955 & 3208 & 5566 & 3145 & 0.313 & 0.037 & 0.327 & 0.039 & 0.255 & 0.041 \\
\hline 00 & 6 & 5508 & 3315 & 4020 & 3230 & 5619 & 3165 & 0.327 & 0.044 & 0.349 & 0.046 & 0.267 & .048 \\
\hline 00 & 7 & 707 & 3367 & 4200 & 283 & 5788 & 3218 & 0.375 & 0.060 & 0.409 & 0.063 & 0.305 & 0.065 \\
\hline $06 / 02 / 2000$ & 8 & 5846 & 3399 & 4318 & 3330 & 5932 & 3260 & 0.408 & 0.070 & 0.449 & 0.078 & 0.338 & 0.079 \\
\hline $06 / 03$ & 9 & 5943 & 3407 & 4413 & 3346 & 5992 & 3275 & 0.432 & 0.073 & 0.481 & 0.084 & 0.351 & 0.084 \\
\hline 0 & 10 & 5903 & 3402 & 4370 & 3325 & 5950 & 3255 & 0.422 & 0.071 & 0.466 & 0.077 & 0.342 & 0.077 \\
\hline 000 & 11 & 5932 & 3411 & 4403 & 3335 & 5956 & 3265 & 0.429 & 0.074 & 0.478 & 0.080 & 0.343 & 0.081 \\
\hline $06 / 06$ & 12 & 5970 & 3425 & 4437 & 3352 & 5995 & 3281 & 0.438 & 0.078 & 0.489 & 0.085 & 0.352 & .086 \\
\hline & 13 & 870 & 3401 & 4341 & 330 & 5886 & 3260 & 0.414 & \begin{tabular}{|l|}
0.071 \\
\end{tabular} & 0.457 & $0.0 / 8$ & \begin{tabular}{|l}
0.327 \\
\end{tabular} & 0.079 \\
\hline 00 & 14 & 5902 & 3408 & 4372 & 3331 & 5914 & 3263 & 0.422 & 0.073 & 0.467 & 0.079 & 0.334 & 0.080 \\
\hline $06 / 09$ & 15 & 5902 & 3407 & 4354 & 3336 & 5928 & 3264 & 0.422 & 0.073 & 0.461 & 0.080 & 0.337 & 0.080 \\
\hline 00 & 16 & 133 & 3467 & 4602 & 3397 & 6147 & 3323 & 0.477 & 0.092 & 0.544 & 0.100 & 0.386 & 100 \\
\hline 0 & 16 & 166 & 3474 & 4642 & 3404 & 6184 & 3333 & 0.485 & 0.094 & 0.558 & 0.102 & 0.395 & 0.103 \\
\hline $06 / 12$ & 18 & 6297 & 3505 & 4752 & 3438 & 6280 & 3359 & 0.517 & 0.104 & 0.595 & 0.113 & 0.416 & 0.112 \\
\hline 00 & 19 & 6231 & 3494 & 4672 & 3422 & 6208 & 3349 & 0.501 & 0.100 & 0.568 & 0.108 & 0.400 & .109 \\
\hline 00 & 20 & 322 & 3452 & 4768 & 3450 & 6286 & 3376 & 0.523 & 0.087 & 0.600 & .117 & 0.418 & .118 \\
\hline $06 / 1$ & 21 & 6336 & 3507 & 4783 & 3445 & 6276 & 3380 & 0.526 & 0.104 & 0.605 & 0.116 & 0.415 & 0.119 \\
\hline $06 / 16$ & 22 & 6342 & 3525 & 4787 & 3462 & 6285 & 3385 & 0.528 & 0.110 & 0.606 & 0.121 & \begin{tabular}{|l|}
0.417 \\
\end{tabular} & 0.120 \\
\hline & 23 & 402 & 3560 & 4899 & 3521 & 6317 & 3400 & 0.542 & 0.121 & 0.644 & 0.140 & 0.425 & 0.125 \\
\hline 00 & 24 & 6585 & 3527 & 5015 & 3564 & 6503 & 3437 & 0.586 & 0.111 & 0.683 & 0.154 & 0.467 & 0.138 \\
\hline $06 / 20 /$ & 26 & 6521 & 3504 & 4956 & 3543 & 6439 & 3420 & 0.571 & 0.103 & 0.663 & 0.147 & 0.452 & 0.132 \\
\hline & 27 & 592 & 3529 & 5024 & 3569 & 6494 & 3450 & 0.588 & \begin{tabular}{|l|}
0.111 \\
\end{tabular} & 0.686 & \begin{tabular}{|l}
0.156 \\
\end{tabular} & \begin{tabular}{|l}
0.465 \\
\end{tabular} & 0.142 \\
\hline $06 / 22$ & 28 & 6460 & 3502 & 4884 & 3536 & 6351 & 3424 & 0.556 & 0.103 & 0.639 & 0.145 & \begin{tabular}{|l|l|}
0.432 \\
\end{tabular} & 0.133 \\
\hline $06 / 23 /$ & 29 & 6420 & 3464 & 4820 & 3517 & 6261 & 3354 & 0.547 & 0.091 & 0.617 & 0.139 & 0.412 & 0.110 \\
\hline 00 & 30 & 456 & 3431 & 4816 & 3416 & 6258 & 3331 & 0.555 & 0.080 & 0.616 & \begin{tabular}{|l|l|}
0.106 \\
\end{tabular} & \begin{tabular}{|l|}
0.411 \\
\end{tabular} & 0.103 \\
\hline $06 / 25$ & 31 & 6434 & 3420 & 5039 & 3564 & 6250 & 3361 & 0.550 & 0.077 & 0.691 & 0.154 & 0.410 & 0.113 \\
\hline $06 / 26$ & 32 & 6639 & 3532 & 5061 & 3589 & 6553 & 3452 & 0.599 & 0.112 & 0.698 & 0.162 & 0.478 & 0.143 \\
\hline & 32 & 722 & 3628 & 5154 & 3609 & 6633 & 3482 & 0.619 & 0.142 & 0.730 & 0.169 & 0.496 & 0.153 \\
\hline $06 / 27 / 2000$ & 33 & 6512 & 3613 & 4879 & 3501 & 6437 & 3412 & 0.569 & 0.138 & 0.637 & 0.134 & 0.452 & 0.129 \\
\hline $06 / 28 / 2000$ & 34 & 6582 & 3498 & 5132 & 3563 & 6500 & 3449 & 0.586 & 0.101 & 0.722 & 0.154 & 0.466 & 0.142 \\
\hline & 35 & 487 & 3503 & 4888 & 3543 & 6369 & 3437 & 0.563 & 0.103 & 0.640 & 0.147 & 0.436 & 0.138 \\
\hline $07 / 06 / 2000$ & 42 & 6656 & 3560 & 5085 & 3587 & 6580 & 3472 & 0.603 & \begin{tabular}{|l|}
0.121 \\
\end{tabular} & 0.706 & 0.162 & \begin{tabular}{|l|}
0.484 \\
\end{tabular} & 0.149 \\
\hline $07 / 07 / 2000$ & 43 & 6543 & 3516 & 4981 & 3487 & 6460 & 3396 & 0.576 & 0.107 & 0.671 & 0.129 & 0.457 & 0.124 \\
\hline $07 / 09$ & 45 & 6582 & 3545 & 4982 & 3569 & 6464 & 3485 & 0.586 & 0.116 & 0.672 & 0.156 & 0.458 & 0.154 \\
\hline $07 / 12 / 2000$ & 48 & 6720 & 3580 & 5115 & 3576 & 6599 & 3450 & 0.619 & 0.127 & 0.716 & 0.158 & 0.488 & 0.142 \\
\hline $07 / 13$ & 49 & 6623 & 3523 & 5014 & 4086 & 6484 & 3432 & 0.596 & 0.109 & 0.683 & 0.323 & 0.462 & 0.136 \\
\hline $07 / 14 / 2000$ & 50 & 6563 & 3502 & 4947 & 3528 & 6368 & 3395 & 0.581 & 0.103 & 0.660 & 0.142 & 0.436 & 0.124 \\
\hline
\end{tabular}




\begin{tabular}{|c|c|c|c|c|c|c|c|c|c|c|c|c|c|}
\hline $7 / 18 / 2000$ & 54 & 802 & 609 & 182 & 606 & 663 & 3457 & .639 & .136 & 0.739 & 0.168 & 0.503 & $\overline{0.144}$ \\
\hline $7 / 22 / 2000$ & 58 & 494 & 496 & 877 & 3528 & 329 & 3410 & .564 & 101 & .637 & 142 & .427 & 129 \\
\hline $7 / 24 / 2000$ & 60 & 6720 & 644 & 5112 & 3569 & 6586 & 3455 & .619 & 0.147 & 0.715 & 0.156 & 0.485 & .144 \\
\hline $7 / 26 / 2000$ & 62 & 6742 & 566 & 5141 & 3586 & 630 & 3464 & .624 & 0.123 & 0.725 & 0.161 & .495 & .147 \\
\hline 00 & 64 & 707 & 539 & 055 & 3570 & 544 & 3439 & .616 & .114 & 0.696 & .156 & .476 & .138 \\
\hline $7 / 30 / 2000$ & 66 & 6752 & 3728 & 5145 & 3606 & 6624 & 3469 & 0.627 & 0.174 & 0.727 & 0.168 & 0.494 & .148 \\
\hline $8 / 04 / 2000$ & 71 & 6807 & 3628 & 5189 & 3631 & 6657 & 3445 & 0.640 & 0.142 & 0.741 & 0.176 & .501 & .140 \\
\hline 000 & 73 & 022 & $\overline{621}$ & 5340 & 3667 & 802 & 3527 & .692 & 0.140 & 0.792 & 0.188 & .534 & .167 \\
\hline $8 / 2000$ & 75 & 6852 & 3600 & 5235 & 3600 & 6685 & 3514 & .651 & 0.134 & 0.757 & 0.166 & 0.508 & .163 \\
\hline $08 / 11 / 2000$ & 78 & 6792 & 3588 & 5177 & 3631 & 6657 & 3506 & 0.636 & 0.130 & 0.737 & 0.176 & 0.501 & 0.161 \\
\hline 2000 & 82 & 6868 & 3614 & 5242 & 3645 & 6714 & 3522 & 0.655 & 0.138 & 0.759 & 0.180 & .514 & .166 \\
\hline $3 / 19 / 2000$ & 86 & 6649 & 3552 & 5048 & 3583 & 6514 & 3468 & 0.602 & 0.118 & 0.694 & 0.160 & 0.469 & .148 \\
\hline $08 / 23 / 2000$ & 90 & 6763 & 3589 & 5143 & 3614 & 6627 & 3502 & 0.629 & 0.130 & 0.726 & 0.170 & 0.495 & 0.159 \\
\hline /28/2 & 95 & 6844 & 3370 & 5215 & 3648 & 6698 & 3528 & 0.649 & 61 & 0.750 & 81 & 11 & .168 \\
\hline$\overline{00}$ & 104 & 698 & 56 & 5092 & 3582 & 6578 & 3452 & 0.614 & .120 & 0.709 & 0.160 & 0.484 & .143 \\
\hline $09 / 11 / 2000$ & 109 & 6964 & 3653 & 5339 & 3690 & 6810 & 3580 & 0.678 & 0.150 & 0.792 & 0.195 & 0.536 & 0.185 \\
\hline $15 / 2000$ & 113 & 6675 & 3662 & 5100 & 3605 & 6583 & 3485 & 0.608 & 0.153 & 0.711 & 0.167 & 0.485 & 0.154 \\
\hline $8 / 2000$ & 16 & 6666 & 3575 & 5061 & 3602 & 6537 & 3572 & 0.606 & 0.126 & 0.698 & 0.166 & 0.474 & 182 \\
\hline $09 / 22 / 2000$ & 120 & 6693 & 3608 & 5089 & 3605 & 6557 & 3511 & 0.612 & 0.136 & 0.708 & 0.167 & 0.479 & 0.162 \\
\hline$/ 25 / 2000$ & 123 & 6590 & 3806 & 4989 & 3589 & 6461 & 3465 & 0.588 & 0.198 & 0.674 & 0.162 & 0.457 & 0.147 \\
\hline $10 / 02 / 2000$ & 130 & 6692 & 3800 & 5091 & 3672 & 6568 & 3571 & 0.612 & 0.196 & 0.708 & 0.189 & 0.481 & 0.182 \\
\hline $10 / 05 / 2000$ & 133 & 6925 & 3674 & 5311 & 3678 & 6866 & 3542 & 0.668 & 0.157 & 0.782 & 0.191 & 0.548 & 0.172 \\
\hline $0 / 08 / 2000$ & 136 & 7112 & 3802 & 5345 & 3692 & 7014 & 3592 & 0.713 & 0.197 & 0.794 & 0.196 & 0.582 & 0.189 \\
\hline $10 / 13 / 2000$ & 141 & 7143 & 3912 & 5381 & 3701 & 7028 & 3601 & 0.721 & 0.232 & 0.806 & 0.199 & 0.585 & 0.192 \\
\hline $10 / 18 / 2000$ & 146 & 7167 & 4008 & 5395 & 3715 & 7075 & 3645 & 0.727 & 0.262 & 0.810 & 0.203 & 0.596 & 0.207 \\
\hline $02 / 08 / 2001$ & 259 & 7225 & 4086 & 5422 & 3826 & 7358 & 3699 & 0.741 & 0.287 & 0.819 & 0.239 & 0.659 & 0.224 \\
\hline $05 / 15 / 2001$ & 355 & 7261 & 4203 & 5436 & 3965 & 7598 & 3821 & 0.749 & 0.323 & 0.824 & 0.284 & 0.714 & 0.265 \\
\hline $07 / 12 / 2001$ & 413 & 7285 & 4312 & 5437 & 4025 & 7656 & 3968 & 0.755 & 0.358 & 0.824 & 0.303 & 0.727 & 0.313 \\
\hline $10 / 17 / 2001$ & 510 & 7306 & 4356 & 5448 & 4086 & 7787 & 4056 & 0.760 & 0.372 & 0.828 & 0.323 & 0.756 & 0.343 \\
\hline $01 / 16 / 2002$ & 601 & 7320 & 4459 & 5486 & 4158 & 7821 & 4115 & 0.763 & 0.404 & 0.841 & 0.347 & 0.764 & 0.362 \\
\hline $04 / 12 / 2002$ & 687 & 7328 & 4521 & 5523 & 4252 & 7869 & 4156 & 0.765 & 0.423 & 0.853 & 0.377 & 0.775 & 0.376 \\
\hline $06 / 04 / 2002$ & 740 & 7340 & 4623 & 5539 & 4365 & 7925 & 4186 & 0.768 & 0.456 & 0.859 & 0.414 & 0.787 & 0.386 \\
\hline $08 / 12 / 2002$ & 809 & 7363 & 4654 & 5551 & 4444 & 7986 & 4237 & 0.774 & 0.465 & 0.863 & 0.439 & 0.801 & 0.403 \\
\hline
\end{tabular}




\section{APPENDIX H}

TEST RESULTS FOR FIBER REINFORCED PLASTIC SPECIMENS FOR HIGHWAY

APPLICATIONS

Table H.1 Recycled Polypropylene Channel Section Bending Test Results

\begin{tabular}{|l|c|c|c|c|c|c|}
\hline $\begin{array}{c}\text { Gage } \\
\text { Location } \\
\text { At Midspan Section }\end{array}$ & Stress & $\begin{array}{c}\text { Maximum } \\
\text { Load } \\
\text { lbs. }\end{array}$ & $\begin{array}{c}\text { Maximum } \\
\text { Stress } \\
\mathbf{p s i}\end{array}$ & $\begin{array}{c}\text { Strain at } \\
\text { Max. Load } \\
\mu \mathbf{s}\end{array}$ & $\begin{array}{c}\text { Max. Strain } \\
\text { Recorded } \\
\mu \mathbf{s}\end{array}$ & $\begin{array}{c}\text { Stiffness, E } \\
\text { psi x 10 }\end{array}$ \\
\hline Side, bottom-most fiber & Tensile & 548 & 13831 & 5854 & 6942 & 2.55 \\
\hline Top, center of width & Compressive & 548 & 6686 & 1839 & 3865 & 2.45 \\
\hline Side, upper-most fiber & Compressive & 548 & 1761 & 453 & 4428 & 2.21 \\
\hline
\end{tabular}

Table H.2 Recycled Polypropylene Trapezoidal Section Bending Test Results

\begin{tabular}{|l|c|c|c|c|c|c|}
\hline $\begin{array}{c}\text { Gage } \\
\text { Location } \\
\text { at Midspan Section }\end{array}$ & Stress & $\begin{array}{c}\text { Maximum } \\
\text { Load } \\
\text { lbs. }\end{array}$ & $\begin{array}{c}\text { Maximum } \\
\text { Stress } \\
\mathbf{p s i}\end{array}$ & $\begin{array}{c}\text { Strain at } \\
\text { Max. Load } \\
\mu \mathbf{s}\end{array}$ & $\begin{array}{c}\text { Max. Strain } \\
\text { Recorded } \\
\mu \mathbf{s}\end{array}$ & $\begin{array}{c}\text { Stiffness, E } \\
\text { psi x } \mathbf{1 0}^{\mathbf{6}}\end{array}$ \\
\hline Bottom, center of width & Tensile & 1001 & 45049 & 11554 & 11554 & 3.16 \\
\hline Top, center of width & Compressive & 1001 & 23722 & 7952 & 7952 & 3.04 \\
\hline Top, side & Compressive & 1001 & 23595 & 7608 & 7608 & 3.02 \\
\hline
\end{tabular}

Table H.3 Plates from Recycled Polypropylene Trapezoidal Section Tension Test Results

\begin{tabular}{|l|c|c|c|c|}
\hline $\begin{array}{c}\text { Gage } \\
\text { Location }\end{array}$ & Grips & $\begin{array}{c}\text { Maximum } \\
\text { Load } \\
\text { lbs. }\end{array}$ & $\begin{array}{c}\text { Maximum } \\
\text { Stress } \\
\text { psi }\end{array}$ & Stiffness, E \\
psi x 10
\end{tabular}


Table H.4 Recycled ABS Box Section Bending Test Results

\begin{tabular}{|c|c|c|c|c|c|c|}
\hline $\begin{array}{c}\text { Type of } \\
\text { Specimen }\end{array}$ & Stress & $\begin{array}{c}\text { Maximum } \\
\text { Load } \\
\text { lbs. }\end{array}$ & $\begin{array}{c}\text { Maximum } \\
\text { Stress } \\
\mathbf{p s i}\end{array}$ & $\begin{array}{c}\text { Strain at } \\
\text { Max. Load } \\
\mu \mathbf{s}\end{array}$ & $\begin{array}{c}\text { Max. Strain } \\
\text { Recorded } \\
\mu \mathbf{s}\end{array}$ & $\begin{array}{c}\text { Stiffness, E } \\
\text { psi x 10 }\end{array}$ \\
\hline $\begin{array}{c}\text { Non-wrapped } \\
\text { 3-point bending }\end{array}$ & Tensile & 290 & 12845 & 3087 & 3087 & 3.86 \\
\cline { 2 - 7 } & Compressive & 280 & 12402 & 7703 & 7703 & 2.28 \\
\hline $\begin{array}{c}\text { Wrapped } \\
\text { 3-point bending }\end{array}$ & Tensile & 504 & 12953 & 4652 & 9309 & 3.24 \\
\cline { 2 - 7 } $\begin{array}{c}\text { Wrapped } \\
\text { 4-point bending }\end{array}$ & Tensile & 1230 & 19733 & 5251 & 5251 & 3.90 \\
\cline { 2 - 7 } & Compressive & 1230 & 19733 & 5852 & 6302 & 2.94 \\
\hline
\end{tabular}

Table H.5 Virgin Vinylester Box Section 3-Point Bending Test Results

\begin{tabular}{|c|c|c|c|c|c|c|}
\hline Specimen No. & Stress & $\begin{array}{c}\text { Maximum } \\
\text { Load } \\
\text { lbs. }\end{array}$ & $\begin{array}{c}\text { Maximum } \\
\text { Stress } \\
\text { Psi }\end{array}$ & $\begin{array}{c}\text { Strain at } \\
\text { Max. Load } \\
\mu \mathbf{s}\end{array}$ & $\begin{array}{c}\text { Max. Strain } \\
\text { Recorded } \\
\mu \mathbf{s}\end{array}$ & $\begin{array}{c}\text { Stiffness, E } \\
\text { psi x 10 }\end{array}$ \\
\hline \multirow{2}{*}{1} & Tensile & 900 & 31735 & 8801 & 8801 & 2.52 \\
\cline { 2 - 7 } & Compressive & 900 & 31735 & 18000 & 18000 & 3.60 \\
\hline \multirow{2}{*}{2} & Tensile & 800 & 28209 & 7837 & 7837 & 1.96 \\
\cline { 2 - 7 } & Compressive & 800 & 28209 & 25250 & 25250 & 3.65 \\
\hline
\end{tabular}

Table H.6 Recycled ABS Box Section Tension Test Results

\begin{tabular}{|l|c|c|c|c|c|}
\hline \multicolumn{1}{|c|}{$\begin{array}{c}\text { Gage } \\
\text { Location }\end{array}$} & $\begin{array}{c}\text { Maximum } \\
\text { Load } \\
\text { lbs. }\end{array}$ & $\begin{array}{c}\text { Maximum } \\
\text { Stress } \\
\mathbf{p s i}\end{array}$ & $\begin{array}{c}\text { Strain at } \\
\text { Max. Load } \\
\boldsymbol{\mu s}\end{array}$ & $\begin{array}{c}\text { Max. Strain } \\
\text { Recorded } \\
\boldsymbol{\mu s}\end{array}$ & $\begin{array}{c}\text { Stiffness, E } \\
\text { psi x 10 }\end{array}$ \\
\hline Front face & 3702 & 6300 & 6600 & 8550 & 3.37 \\
\hline Lateral face & 3202 & 6300 & 6450 & 8100 & 3.04 \\
\hline AVERAGE & & & & & 3.21 \\
\hline
\end{tabular}


Table H.7 Recycled ABS Short Plates Cut from Sheets Bending Test Results

\begin{tabular}{|c|c|c|c|c|c|c|}
\hline $\begin{array}{c}\text { Specimen } \\
\text { No. }\end{array}$ & Stress & $\begin{array}{c}\text { Maximum } \\
\text { Load } \\
\text { Ibs. }\end{array}$ & $\begin{array}{c}\text { Maximum } \\
\text { Stress } \\
\text { psi }\end{array}$ & $\begin{array}{c}\text { Strain at } \\
\text { Max. Load } \\
\mu \mathbf{s}\end{array}$ & $\begin{array}{c}\text { Max. Strain } \\
\text { Recorded } \\
\mu \mathbf{s}\end{array}$ & $\begin{array}{c}\text { Stiffness, E } \\
\text { psi x 10 }\end{array}$ \\
\hline \multirow{2}{*}{1} & Tensile & 195 & 27240 & 14300 & 14300 & 2.59 \\
\cline { 2 - 7 } & Compressive & 195 & 27240 & 16911 & 16911 & 2.33 \\
\hline \multirow{2}{*}{2} & Tensile & 200 & 28745 & - & - & - \\
\cline { 2 - 7 } & Compressive & 200 & 28745 & - & - & - \\
\hline
\end{tabular}

Table H.8 Recycled ABS Long Plates Cut from Sheets Bending Test Results

\begin{tabular}{|c|c|c|c|c|c|c|}
\hline $\begin{array}{c}\text { Specimen } \\
\text { No. }\end{array}$ & Stress & $\begin{array}{c}\text { Maximum } \\
\text { Load } \\
\text { Ibs. }\end{array}$ & $\begin{array}{c}\text { Maximum } \\
\text { Stress } \\
\text { Psi }\end{array}$ & $\begin{array}{c}\text { Strain at } \\
\text { Max. Load } \\
\mu \mathbf{S}\end{array}$ & $\begin{array}{c}\text { Max. Strain } \\
\text { Recorded } \\
\mu \mathbf{s}\end{array}$ & $\begin{array}{c}\text { Stiffness, E } \\
\text { psi x 10 }\end{array}$ \\
\hline \multirow{2}{*}{1} & Tensile & 160 & 39815 & 13734 & 13734 & 3.01 \\
\cline { 2 - 7 } & Compressive & 160 & 39815 & 15118 & 15118 & 2.92 \\
\hline \multirow{2}{*}{2} & Tensile & 160 & 39815 & 13718 & 13718 & 3.04 \\
\cline { 2 - 7 } & Compressive & 160 & 39815 & 14832 & 14832 & 2.90 \\
\hline
\end{tabular}

Table H.9 Recycled ABS Sheets Tension Test Results

\begin{tabular}{|c|c|c|c|}
\hline Specimen No. & $\begin{array}{c}\text { Maximum } \\
\text { Load } \\
\text { lbs. }\end{array}$ & $\begin{array}{c}\text { Maximum } \\
\text { Stress } \\
\text { psi }\end{array}$ & psiffness, E $\mathbf{1 0}^{\mathbf{6}}$ \\
\hline 1 & 2261 & 20727 & 2.68 \\
\hline 2 & 2669 & 23194 & 2.69 \\
\hline
\end{tabular}

Table H.10 Recycled ABS Sheets Compression Test Results

\begin{tabular}{|c|c|c|c|c|}
\hline Specimen No. & $\begin{array}{c}\text { Maximum } \\
\text { Load } \\
\text { lbs. }\end{array}$ & $\begin{array}{c}\text { Maximum } \\
\text { Stress } \\
\mathbf{p s i}\end{array}$ & $\begin{array}{c}\text { Strain at } \\
\text { Max. Load } \\
\mu \mathbf{s}\end{array}$ & Stiffness, E \\
psi x 10
\end{tabular}


Table H.11 Recycled ABS Belt-Type Specimens Tension Test Results

\begin{tabular}{|c|c|c|c|c|c|}
\hline $\begin{array}{c}\text { Specimen } \\
\text { Type }\end{array}$ & $\begin{array}{c}\text { Specimen } \\
\text { No. }\end{array}$ & $\begin{array}{c}\text { Maximum } \\
\text { Load } \\
\text { Ibs. }\end{array}$ & $\begin{array}{c}\text { Maximum } \\
\text { Stress } \\
\text { psi }\end{array}$ & $\begin{array}{c}\text { Strain at } \\
\text { Max. Load } \\
\boldsymbol{\mu s}\end{array}$ & Stiffness, E \\
psi x 10 $\mathbf{1 0}^{\mathbf{6}}$ \\
\hline \multirow{3}{*}{$\mathrm{A}$} & $\mathrm{A} 1$ & 495 & 25085 & 9909 & 2.86 \\
\cline { 2 - 6 } & $\mathrm{A} 2$ & 618 & 34497 & 12662 & 2.98 \\
\cline { 2 - 6 } & AVERAGE & & 29791 & & 2.92 \\
\hline \multirow{3}{*}{$\mathrm{B}$} & $\mathrm{B} 1$ & 497 & 36808 & 14320 & 2.68 \\
\cline { 2 - 6 } & $\mathrm{B} 2$ & 517 & 37482 & 13605 & 2.62 \\
\cline { 2 - 6 } & AVERAGE & & 37145 & & 2.65 \\
\hline \multirow{2}{*}{$\mathrm{C}$} & $\mathrm{C} 1$ & 332 & 20771 & 9001 & 2.17 \\
\cline { 2 - 6 } & $\mathrm{C} 2$ & 439 & 28053 & 13171 & 2.02 \\
\cline { 2 - 6 } & AVERAGE & & 24412 & & 2.09 \\
\hline
\end{tabular}

Table H.12 Wooden Blocks from Rubber-Wood Block Model Compression Test Results

\begin{tabular}{|c|c|c|c|c|c|}
\hline $\begin{array}{c}\text { Specimen } \\
\text { Type }\end{array}$ & $\begin{array}{c}\text { Specimen } \\
\text { No. }\end{array}$ & $\begin{array}{c}\text { Maximum } \\
\text { Load } \\
\text { lbs. }\end{array}$ & $\begin{array}{c}\text { Maximum } \\
\text { Stress } \\
\text { psi }\end{array}$ & $\begin{array}{c}\text { Strain at } \\
\text { Max. Load } \\
\mu \mathbf{s}\end{array}$ & Stiffness, E \\
psi x 10
\end{tabular}

Table H.13 Wooden Specimens from Rubber-Wood Block Model Impact Test Results

\begin{tabular}{|c|c|c|c|c|c|c|}
\hline $\begin{array}{c}\text { Specimen } \\
\text { No. }\end{array}$ & Thickness & $\begin{array}{c}\text { Indicated } \\
\text { Impact } \\
\text { Strength } \\
\text { ft-lb }\end{array}$ & Correction & $\begin{array}{c}\text { Corrected } \\
\text { Impact } \\
\text { Strength } \\
\text { in }\end{array}$ & $\begin{array}{c}\text { Impact } \\
\text { Strength }\end{array}$ & $\begin{array}{c}\text { Failure } \\
\text { Type } \\
\text { ft-lb/in }\end{array}$ \\
\hline 1 & 0.26 & 0.67 & 0.39 & 0.28 & 1.10 & $\mathrm{C}$ \\
\hline 2 & 0.27 & 0.58 & 0.41 & 0.17 & 0.62 & $\mathrm{C}$ \\
\hline 3 & 0.25 & 0.85 & 0.37 & 0.48 & 1.96 & $\mathrm{H}$ \\
\hline 4 & 0.24 & 1.02 & 0.34 & 0.68 & 2.85 & $\mathrm{H}$ \\
\hline 5 & 0.28 & 0.45 & 0.43 & 0.02 & 0.07 & $\mathrm{C}$ \\
\hline 6 & 0.29 & 1.52 & 0.27 & 1.25 & 4.31 & $\mathrm{H}$ \\
\hline AVERAGE & & & & & 1.82 & \\
\hline
\end{tabular}


Table H.14 ABS-Rubber Pull-Out Test Results

\begin{tabular}{|c|c|c|c|c|c|}
\hline $\begin{array}{c}\text { Molding } \\
\text { Process }\end{array}$ & $\begin{array}{c}\text { Curing } \\
\text { Time }\end{array}$ & Primer & $\begin{array}{c}\text { Maximum } \\
\text { Load } \\
\text { Ibs }\end{array}$ & $\begin{array}{c}\text { Maximum } \\
\text { Stress } \\
\text { psi }\end{array}$ & $\begin{array}{c}\text { Displacement } \\
\text { at Max. Load } \\
\text { in }\end{array}$ \\
\hline Single compression & 4 & Yes & 133 & 38.7 & 0.146 \\
\hline Remolded & 6 & Yes & 281 & 82.1 & 0.221 \\
\hline Single compression & 8 & Yes & 267 & 78.9 & 0.232 \\
\hline Remolded & 8 & Yes & 297 & 87.9 & 0.313 \\
\hline Single compression & 8 & No & 73 & 20.9 & 0.090 \\
\hline Remolded & 8 & No & 126 & 36.0 & 0.114 \\
\hline
\end{tabular}

Table H.15 Fiber Reinforced Plastics Ignition Test Results

\begin{tabular}{|l|l|c|c|c|c|}
\hline \multicolumn{1}{|c|}{ Section } & \multicolumn{1}{|c|}{ Resin } & $\begin{array}{c}\text { Weight } \\
\text { of } \\
\text { Fibers } \\
\text { gr }\end{array}$ & $\begin{array}{c}\text { Fiber } \\
\text { Volume }\end{array}$ & $\begin{array}{c}\text { Specimen } \\
\text { Volume }\end{array}$ & $\begin{array}{c}\text { Fiber } \\
\text { Volume } \\
\text { Fraction } \\
\text { in }\end{array}$ \\
\hline Trapezoidal & Recycled Polypropylene & 9.28 & 0.199 & 0.484 & 41 \\
\hline Channel & Recycled Polypropylene & 4.40 & 0.094 & 0.221 & 43 \\
\hline Box & Recycled ABS & 0.60 & 0.013 & 0.052 & 25 \\
\hline Box & Virgin Vinylester & 9.68 & 0.208 & 0.503 & 41 \\
\hline Wrapped Box & Recycled ABS & 5.68 & 0.122 & 0.556 & 22 \\
\hline Sheet & Recycled ABS & 1.27 & 0.027 & 0.107 & 25 \\
\hline Belt-type A & Recycled ABS & 0.30 & 0.006 & 0.032 & 20 \\
\hline Belt-type B & Recycled ABS & 0.38 & 0.008 & 0.034 & 24 \\
\hline Belt-type B & Recycled ABS & 0.22 & 0.005 & 0.025 & 19 \\
\hline
\end{tabular}




\section{APPENDIX I \\ THEORETICAL CALCULATION OF TENSILE MODULUS FOR RANDOMLY ORIENTED CHOPPED FIBER REINFORCED PLASTICS}

The following empirical formula proposed by (Agarwal and Broutman, 1980) can be used to estimate the tensile module of a randomly oriented chopped fiber reinforced plastic:

$\mathrm{E}_{\text {random }}=(3 / 8) \mathrm{E}_{\mathrm{L}}+(5 / 8) \mathrm{E}_{\mathrm{T}}$

Where,

$\mathrm{E}_{\text {random }}=$ Tensile module of reinforced plastic

$\mathrm{E}_{\mathrm{L}}=$ Longitudinal module

$\mathrm{E}_{\mathrm{T}}=$ Transverse module

Longitudinal and transverse modulus can be determined by the Halpin-Tsai equation (Agarwal and Broutman, 1980). Calculations for our ABS reinforced specimens are as follows:

$E_{L}=E_{m}\left[\left(1+(2 L / d) \eta_{L} V_{f}\right) /\left(1-\eta_{L} V_{f}\right)\right]$

Where,

$\mathrm{E}_{\mathrm{f}}=$ Fiber module, $72.4 \mathrm{Gpa}\left(10.5 \times 10^{6} \mathrm{psi}\right)$

$\mathrm{E}_{\mathrm{m}}=$ Matrix module, $2.21 \mathrm{Gpa}\left(0.321 \times 10^{6} \mathrm{psi}\right)$

$\mathrm{V}_{\mathrm{f}}=$ Fiber volume fraction $=12 \%$

$\mathrm{L}=$ Fiber length, $4 \mathrm{~mm}$

$\mathrm{d}=$ Fiber diameter, $0.014 \mathrm{~mm}$

$\eta_{L}=\left[\left(E_{f} / E_{m}\right)-1\right] /\left[\left(E_{f} / E_{m}\right)+(21 / d)\right]$ (Agarwal and Broutman, 1980)

replacing,

$\eta_{L}=[(72.4 / 2.21)-1] /[(72.4 / 2.21)+(2 \times 4 / 0.014)]=0.053$ 
and

$\left.E_{L}=2.21[(1+(1 \times 4 / 0.014) 0.053 \times 0.12) / 1-0.053 \times 0.12)\right]=9.80 \mathrm{Gpa}\left(1.42 \times 10^{6} \mathrm{psi}\right)$

Transverse module is given by

$\mathrm{E}_{\mathrm{T}}=\mathrm{E}_{\mathrm{m}}\left[\left(1+\left(2 \eta_{\mathrm{T}} \mathrm{V}_{\mathrm{f}}\right) /\left(1-\eta_{\mathrm{T}} \mathrm{V}_{\mathrm{f}}\right)\right]\right.$

Where,

$\eta_{T}=\left[\left(E_{f} / E_{m}\right)-1\right] /\left[\left(E_{f} / E_{m}\right)+2\right]$ (Agarwal and Broutman, 1980)

Thus,

$\eta_{\mathrm{T}}=[(72.4 / 2.21)-1] /[(72.4 / 2.21)+2]=0.914$

and

$\left.\mathrm{E}_{\mathrm{T}}=2.21[(1+2.21 \times 0.914 \times 0.12) / 1-0.914 \times 0.12)\right]=2.98 \mathrm{Gpa}\left(0.43 \times 10^{6} \mathrm{psi}\right)$

And finally, the the random modulus is calculated as:

$\mathrm{E}_{\text {random }}=(3 / 8) \times 9.80+(5 / 8) \times 2.98=5.53 \mathrm{Gpa}\left(0.802 \times 10^{6} \mathrm{psi}\right)$ 